\title{
Electron Emission from Metastable Carbon Monoxide Molecules at Adsorbate Covered Au(111) Surfaces
}

\author{
Dissertation \\ Zur Erlangung des mathematisch-naturwissenschaftlichen Doktorgrades \\ Doctor of Philosophy \\ der Georg-August-Universität Göttingen \\ im Promotionsprogramm Chemie \\ der Georg-August University School of Science (GAUSS)
}

Vorgelegt von

Daniel Paul Engelhart

Aus Aberdeen, South Dakota, USA

Göttingen, 2015.06.06 

Betreuungsausschuss

Prof. Alec M. Wodtke, Dynamics at Surfaces, Intitute for Physical Chemistry/ Max-Planck-Institute for Biophysical Chemistry

Prof. Dirk Schwarzer, Dynamics at Surfaces, Institute for Physical Chemistry/ Max-Planck-Institute for Biophysical Chemistry

Mitglieder der Prüfungskommission

Referent: Prof. Alec M. Wodtke, Dynamics at Surfaces, Intitute for Physical Chemistry/ Max-Planck-Institute for Biophysical Chemistry

Korreferent: Prof. Dirk Schwarzer, Dynamics at Surfaces, Institute for Physical Chemistry/ Max-Planck-Institute for Biophysical Chemistry

Weitere Mitglieder der Prüfungskommission:

Prof. Jörg Schröder, Institute for Physical Chemistry

Prof. Martin Suhm, Institute for Physical Chemistry

Prof. Jürgen Troe, Institute for Physical Chemistry

PD Dr. Thomas Zeuch, Abteilung Suhm, Institute for physical chemistry 


\begin{abstract}
Understanding the fundamentals of energy transfer between molecules and surfaces is of profound importance in modern chemistry. Here, I investigate an important energy transfer process, electron transfer (ET), which plays a key role in many surface processes such as photochemistry and light harvesting using dye-sensitized photovoltaics. I probe the dynamics of ET by studying what happens when electronically excited molecules collide with surfaces. In particular I studied scattering of $\mathrm{CO}\left(\mathrm{a}^{3} \Pi_{1}\right)$, referred to as $\mathrm{CO}^{*}$, from clean and adsorbate covered $\mathrm{Au}(111)$. Because the internal energy of $\mathrm{CO}^{*}$ exceeds the work function of the $\mathrm{Au}(111)$ surface, $\mathrm{CO}^{*}$ quenching at the surface can lead to electron emission and the yield of electron emission provides a sensitive probe of the energy transfer mechanisms involved.
\end{abstract}

These studies required the use of a unique, highly versatile molecule-surface scattering apparatus which was designed and built during the course of this work. The instrument consists of a Stark decelerator-based molecular beam source for $\mathrm{CO}^{*}$, a cryogenic sample mount, detectors for ions and electrons, surface preparation equipment and three laser systems used for the preparation and state-selective detection of scattered molecules.

The electron emission probability, $\gamma$, depends in an interesting way on the initial vibrational state of the molecule and the coverage of rare gas adsorbates. $\gamma$ is $0.13 \pm 0.05$ for $\mathrm{CO}^{*}(v=0)$ on atomically clean $\mathrm{Au}(111), 0.19$ for a mix of vibrationally excited $\mathrm{CO}^{*}(v=1,2,3)$, and 0.34 for a mix of $\mathrm{CO}^{*}(v=4,5,6)$. Surprisingly, scattering $\mathrm{CO}^{*}$ in its ground vibrational state from Ar, $\mathrm{Kr}$, or $\mathrm{Xe}$ covered $\mathrm{Au}(111)$ increases rather than decreases $\gamma ; \gamma$ is approximately 0.5 for monolayer coverage of all three gases and approaches unity upon adsorption of additional Ar and $\mathrm{Kr}$.

Conventionally, metastable quenching is explained in terms of an Auger process. This mechanism predicts lower values of $\gamma$ than I observe, a reduction in $\gamma$ with coverage of rare gas adsorbates, and almost no effect of initial vibrational state. Therefore, an alternative mechanism is proposed in which electron emission proceeds via formation of a short-lived anion. In the proposed mechanism, an electron transfers from the gold surface to $\mathrm{CO}^{*}$ as the molecule approaches the surface, forming an anion. Subsequently, the anion relaxes to neutral CO on a femtosecond time scale by auto-detachment. The electron emitted from the molecule either 
escapes into vacuum and is detected or is absorbed by the surface. In contrast to the Auger mechanism, the magnitude and trends in the measured values of $\gamma$ can all be understood in the context of the anion mediated de-excitation model.

Favorable overlap of the $\mathrm{CO}^{*}$ molecular orbitals with the wave functions of the metal are a key factor in understanding the high electron emission probability. The increase in $\gamma$ with vibrational excitation arises because the ground state $\left({ }^{2} \Pi\right)$ of $\mathrm{CO}^{-}$is resonant with $\mathrm{CO}^{*}$ near a surface only at extended bond lengths. $\mathrm{CO}^{*}$ in higher vibrational states spends more time with extended $\mathrm{C}-\mathrm{O}$ bond lengths, thereby increasing the efficiency of the first electron transfer step. The increased efficiency of the first electron step leads to initial charge transfer (ionization) at greater molecule-surface distances, followed immediately by auto-detachment; the emitted electron, therefore, experiences weaker image interaction with the surface and has a higher probability of escaping into vacuum.

The adsorbate induced increase in $\gamma$ can also be understood in terms of the anion mediated deexcitation model. This increase arises due to an increase in the electron reflection probability as closed-shell noble gases are adsorbed on a metal surface. With increased adsorbate coverage, the probability that an electron emitted from the molecule toward the surface is scattered back into vacuum increases, thus increasing the observed electron signal. After some critical adsorbate coverage, the initial electron transfer step from surface to molecule becomes inefficient and electron emission decreases with additional adsorbate coverage.

In addition, I performed thermal desorption measurements of $\mathrm{Ar}, \mathrm{Kr}, \mathrm{Xe}, \mathrm{N}_{2}, \mathrm{NO}, \mathrm{C}_{2} \mathrm{H}_{2}$ and $\mathrm{SF}_{6}$ from $\mathrm{Au}(111)$ in order to characterize the temperature dependence of adsorbate coverage and to measure desorption activation energies, which are excellent proxies for binding energies in the low temperature regime. Binding energy scales with adsorbate polarizability, supporting the conclusion that the surface-adsorbate bonds are dominated by dispersion forces.

Through measurements of $\gamma$, I have developed a better understanding of electron transfer processes at surfaces. Quenching of $\mathrm{CO}^{*}$ proceeds by formation of a transient anion and subsequent auto-detachment. These measurements provide important reference data for theoretical models describing dispersion forces and electron transfer at surfaces. I hope this work inspires continued investigations into dynamics at interfaces. 


\section{Contents}

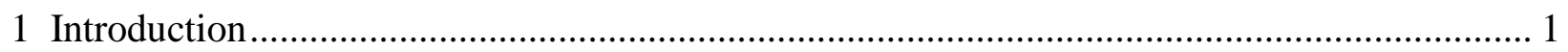

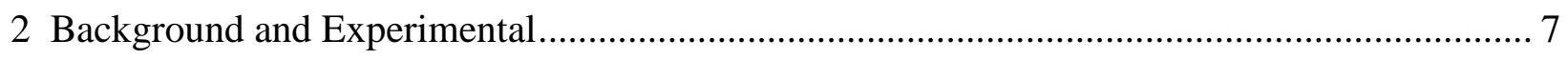

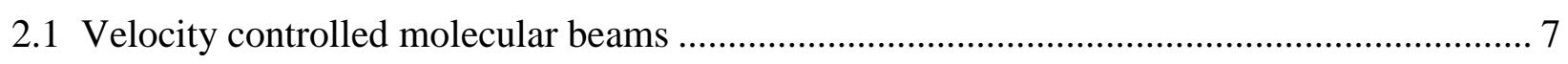

2.1.1 Production of carrier gas-free, state-selected molecular beams...................................... 8

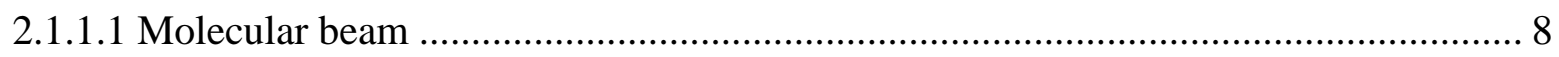

2.1.1.2 Production of metastable CO ............................................................................ 10

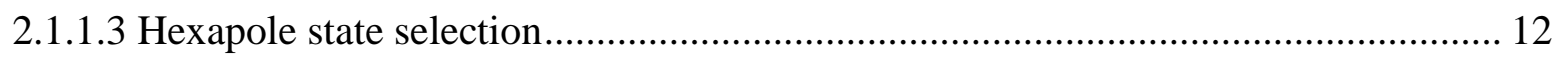

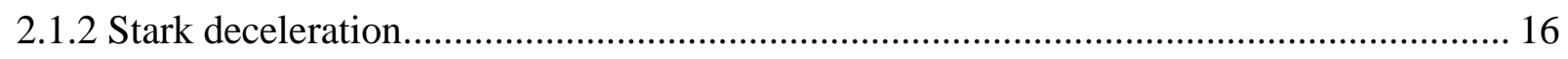

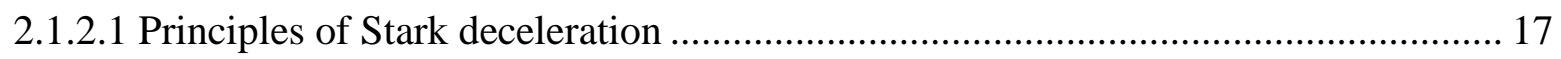

2.1.2.2 Construction of a bakeable Stark decelerator .......................................................... 26

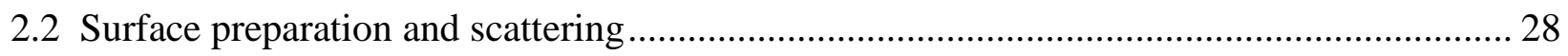

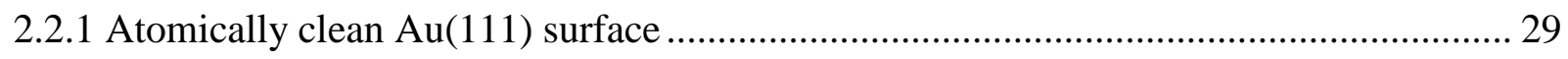

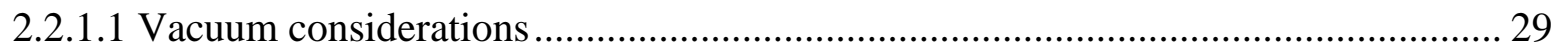

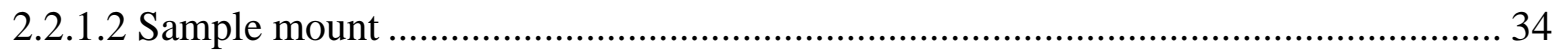

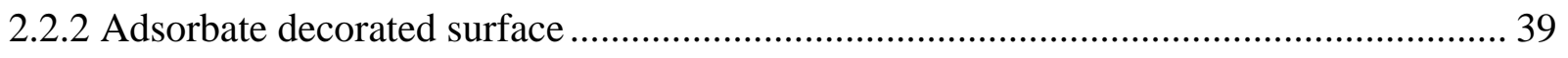

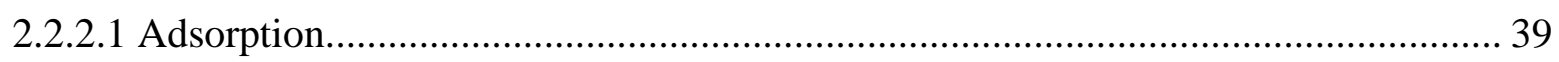

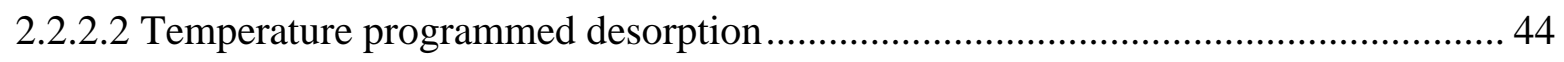

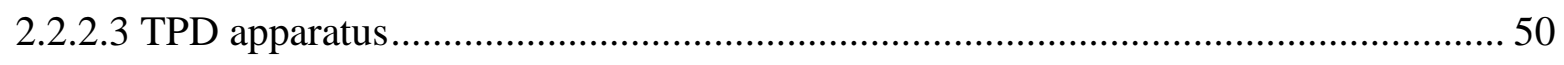

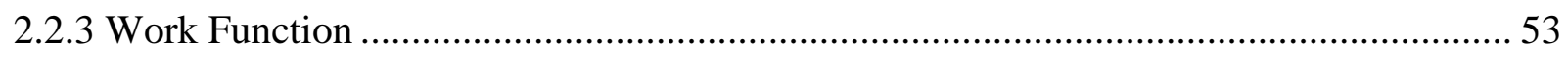

2.2.3.1 Work Function and effect of adsorbates ............................................................... 53

2.2.3.2 Kelvin probe work function measurements ............................................................... 55

2.2.4 Scattering and detection .......................................................................................... 59

2.2.4.1 Photomultiplier tube detection ............................................................................. 59

2.2.4.2 REMPI ionization and charged particle detection .................................................. 60 


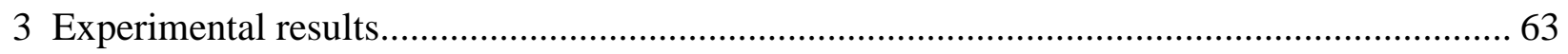

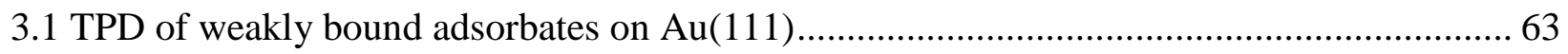

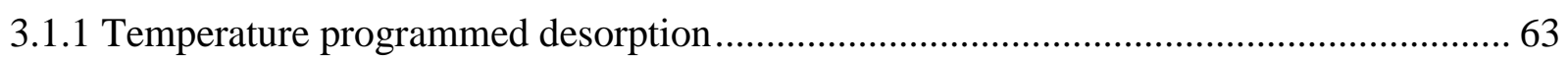

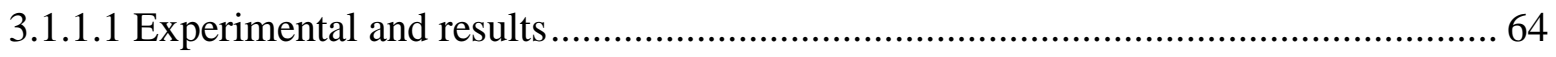

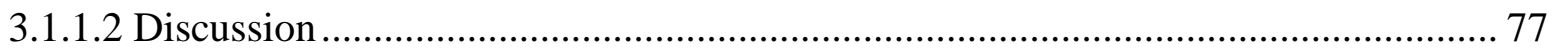

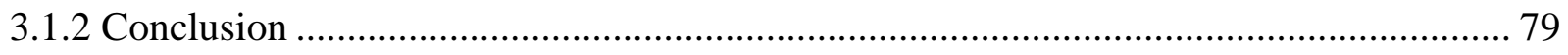

3.2 Electron emission from clean and adsorbate covered $\mathrm{Au}(111)$ surface .................................. 80

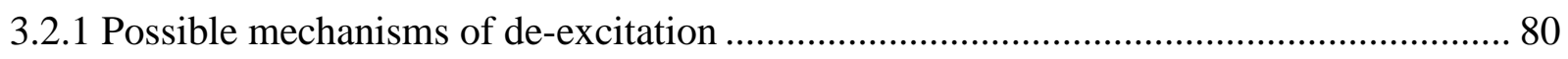

3.2.2 Absolute Electron Emission Probability .......................................................................... 87

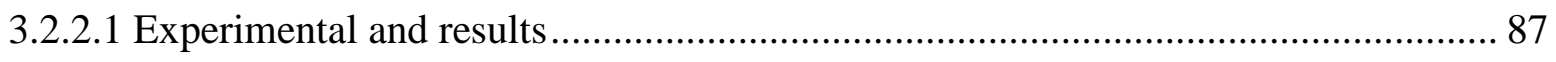

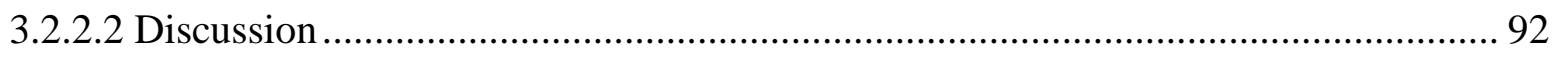

3.2.3 Vibrational enhancement of electron emission.............................................................. 94

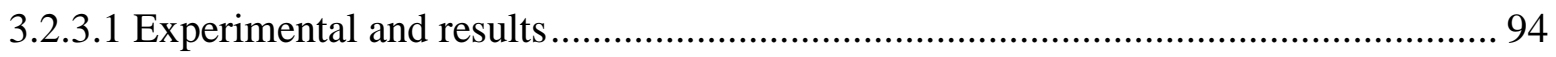

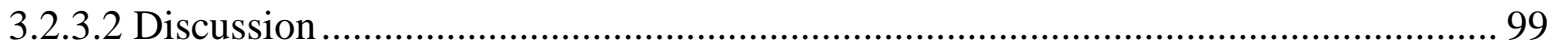

3.2.4 Electron emission from adsorbate covered $\mathrm{Au}(111)$ surfaces .................................... 103

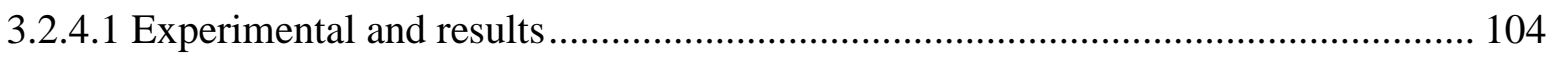

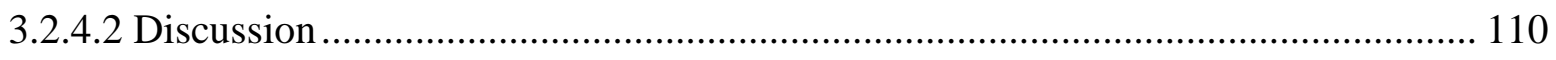

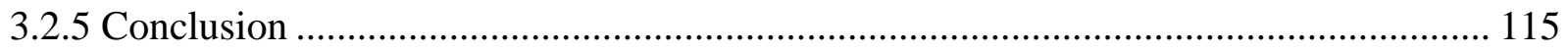

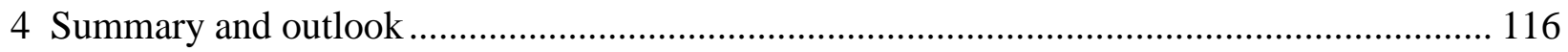

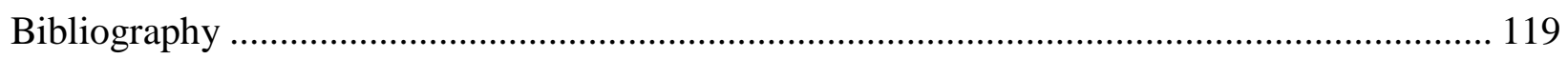

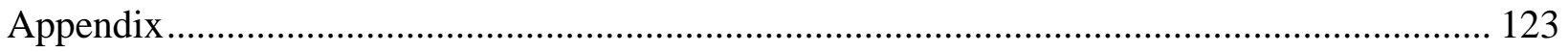

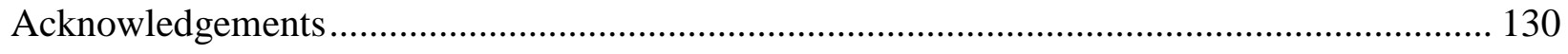

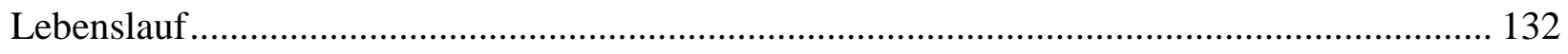




\section{Introduction}

Energy transfer at interfaces is of tremendous importance for a great variety of phenomena in the industrial and engineering world. Production of chemicals such as fertilizer and pharmaceuticals as well as a host of applications in the petrochemical industry all rely on heterogeneous catalysis in which reactants in one phase react in the presence of a catalyst in a different phase. This typically means gases or liquids reacting in the presence of a solid catalyst. The reactions between gas- or liquid-phase reactants occurs with a lower activation energy on the surface of the catalyst. These surface catalyzed reactions can proceed via two major mechanisms. In a Langmuir-Hinshelwood type mechanism, two different reactants, A and B, adsorb to a surface. While adsorbed, the participating reactants encounter one another, bond, and desorb as molecule AB. In an Eley-Rideal type reaction, only reactant A adsorbs to the surface. Reactant B encounters $\mathrm{A}$ on the surface, reacts to form $\mathrm{AB}$, and desorbs immediately. Clearly, one crucial step in either process is the initial interaction of one or both reactants with the surface. What factors affect adsorption probability? How strongly are the reactants bound to the surface? How mobile is the adsorbate on the surface? Do the reactants exchange energy with the surface? Figure 1 illustrates the broad range of possible primary interactions when a gas-phase molecule strikes a surface.

The answers to these and other questions allow for the design of newer, more efficient catalysts and for the streamlining of existing processes. Due to the fundamental nature of the questions, the answers are also applicable to many other fields including aerodynamics, tribology, heat transfer, and materials processing. In order to learn the answers, it is necessary to design experiments which can differentiate between different channels of energy exchange in a system. To understand the fundamental steps which comprise a surface reaction, one must "freeze out" competing channels and examine the effect of each degree of freedom of both reactant and surface. In this respect, a molecule in the ground electronic, vibrational and rotational state approaching an atomically perfect surface with very little translational energy represents the perfect microscopic laboratory in which to study interfacial energy transfer by exciting individual degrees of freedom and observing the results. 


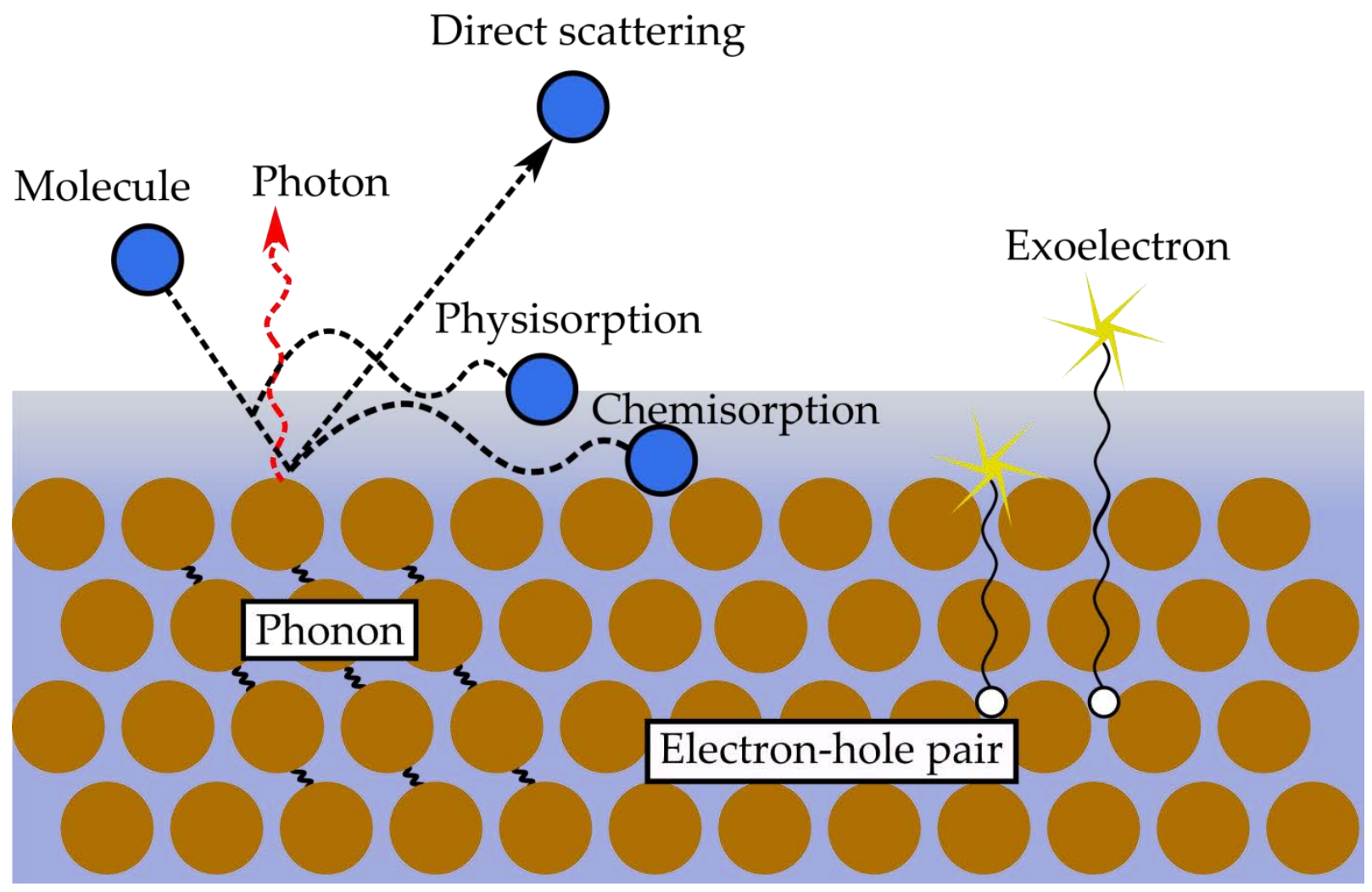

Figure 1 Overview of primary processes that may occur during a molecule-surface encounter. As a molecule approaches the surface, it can scatter directly, physisorb, or chemisorb depending on the magnitude of energy lost to the surface. Energy can be dissipated to nuclei, resulting in phonon excitation, or to electrons, creating electron-hole pairs or leading to electron emission from the surface. Nuclei are represented by brown circles and the electron gas by the purple background.

Studying well characterized surfaces under ultra-high vacuum (UHV) conditions has allowed characterization of the atomic structure of clean and adsorbate covered surfaces. For example, different channels of energy dissipation, such as electron-hole pair excitation and phonon creation can be distinguished. By probing well-characterized surfaces with electrons and photons the physical properties of interfaces can be observed. These techniques, such as low energy electron diffraction (LEED), ${ }^{1,2}$ scanning tunneling microscopy (STM), ${ }^{3,4}$ and x-ray photoelectron spectroscopy (XPS) ${ }^{5,6}$ have led to many advances in our understanding of the behavior of atoms and molecules on surfaces in general and catalysis in particular. For example, this work has led to a clear understanding of the energetics of the Haber-Bosch process in which 
$\mathrm{N}_{2}$ and $\mathrm{H}_{2}$ react on the surface of an iron catalyst to form $\mathrm{NH}_{3}{ }^{7}$ The reaction in the gas phase and in the presence of a catalyst is shown in Figure 2.

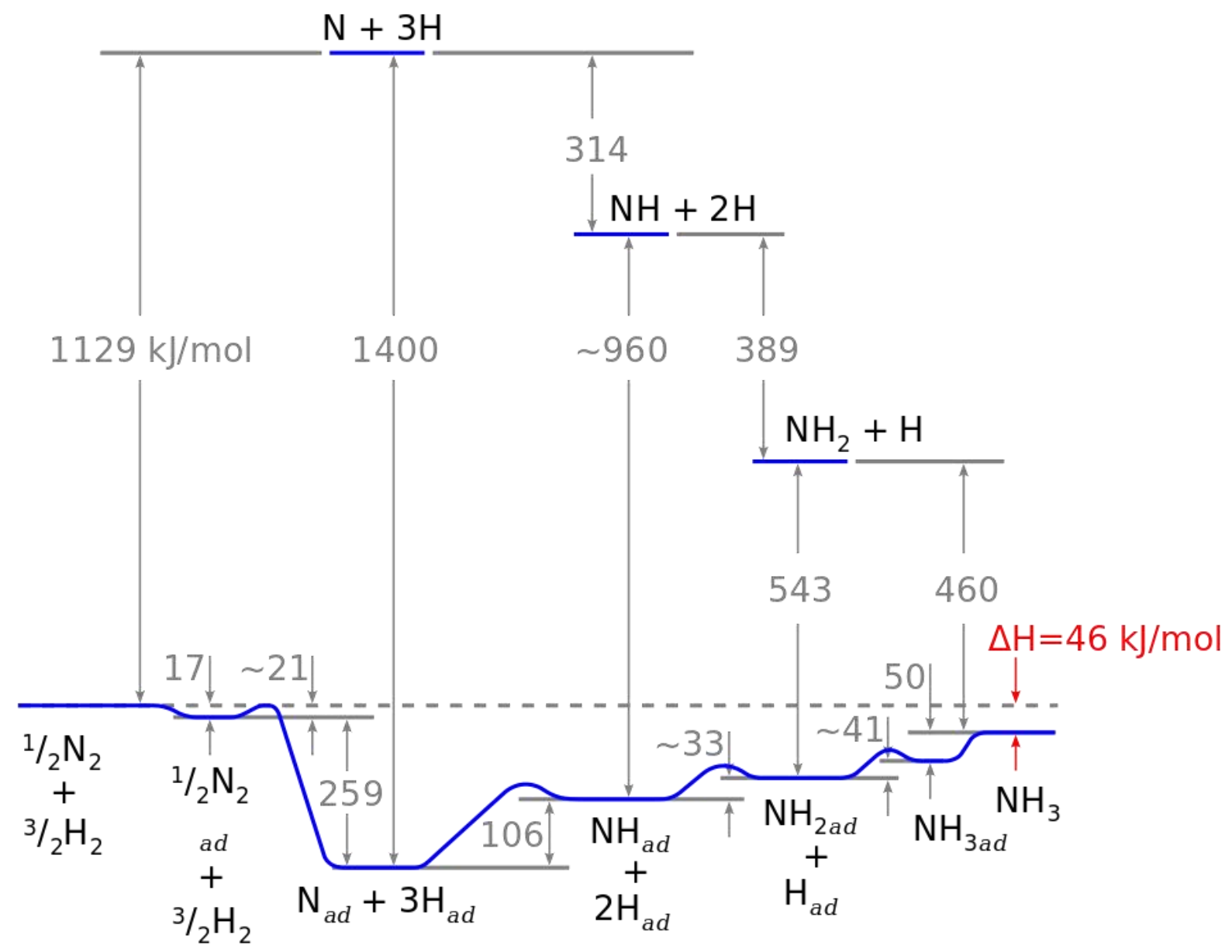

Figure 2 Diagram of ammonia synthesis illustrating the energetic differences between the reaction in the presence of an iron catalyst (lower) and in the gas phase (upper) reaction. ${ }^{7}$

Well-characterized beams of atoms can also be used as probes of surfaces. By scattering atoms from surfaces and measuring the energy of the scattered particles, a great deal can be learned about energy exchanged during the scattering process. If a long lived electronically excited (metastable) atom transfers enough energy to an electron in the surface, an electron can be emitted into vacuum during the scattering event. ${ }^{8}$ Measurement of the probability of electron emission and the kinetic energy distribution of the emitted electrons (exoelectrons) provides information about the density of states at a surface. This technique is knows as metastable 
quenching spectroscopy (MQS). Because massive atoms cannot easily penetrate deeply into the bulk of a solid, this tool is particularly sensitive to phenomena which occur at the surface and to the density of states of adsorbates bound to a surface..$^{8-18}$

As mentioned above, one driving force behind these studies is the understanding of chemical reactions which occur at surfaces, forming new molecules. Molecules are comprised of multiple atoms and can store energy in internal degrees of freedom not available to atoms. Scattering collimated beams of molecules in a chosen quantum state with a narrow distribution of velocities from well characterized surface and examining exoelectrons and the quantum state and translational energy of the scattered molecule enables the experimentalist to examine the effect of every degree of freedom of both molecule and surface. ${ }^{19,}{ }^{20}$ Molecule-surface scattering experiments are an important step toward realizing the microscopic laboratory introduced above.

Molecule-surface scattering studies allow discrimination between direct scattering and trapping-desorption interactions, loss of energy from the molecule to the surface, excitation of the molecule by the surface, and different energy dissipation channels between molecule and surface. Because these experiments can be conducted on simple systems with quantum state resolution they serve as important benchmarks for emerging theory. One important theoretical approach used in gas-phase dynamics is the Born-Oppenheimer approximation (BOA). The BOA suggests that energetics of electrons and nuclei can be decoupled because the electron clouds adjust instantaneously to any given set of nuclear coordinates. This simplifies calculations because the electronic and nuclear repulsion energy can be calculated for several fixed nuclear geometries. This underlies the concept of the potential energy surface (PES). The BOA is only valid if the electronic states of the system are widely separated in energy. It can be expected that for metal solids that calculations based on the BOA may not be physically reasonable. ${ }^{21-23}$

Indeed, many cases of BOA breakdown have been reported. When highly vibrationally excited NO is scattered from a $\mathrm{Au}(111)$ surface, several quanta of vibrational energy are lost in a single scattering event. Due to the short interaction time associated with direct scattering, the loss of so much vibrational energy to phonons is not physically reasonable due to frequency mismatch. This suggests that vibrational (nuclear) energy must couple efficiently with the creation of electron-hole pairs in the metal. By lowering the work function of $\mathrm{Au}(111)$ sufficiently with adsorbed Cs, exoelectrons can be observed. The vibrational energy loss 
threshold for electron emission coincides with the surface work function. This implies that several quanta of vibrational energy of the molecule can couple to a single electron in the surface. The emission of electrons was found to depend on the inverse of the incident molecule's velocity, indicating that the time spent by the molecule in the region of interaction near the surface plays a crucial role in the energy transfer process. ${ }^{24-27}$ Many other examples of BOA breakdown have been reported in recent years, suggesting the need to move beyond adiabatic calculations to describe the interaction of molecules with surfaces. ${ }^{21-23,28-32}$

The interpretation of any experiment is limited by the precision with which the experimentalist can control the system under scrutiny. Molecular beams provide a means to create beams of molecules with a very narrow distribution of vibrational and rotational energy near the ground state and a very low translational energy within the beam frame of reference, i.e. a narrow velocity spread. There is, however, a practical lower limit to the translational energy of standard molecular beams.

By using time-varying strong electric fields to manipulate neutral polar molecules, a beam of carrier gas-free molecules in a single quantum state can be prepared with an extremely narrow velocity distribution and arbitrarily low translation energies. The first successful implementation of a molecular decelerator to decelerate a beam of $\mathrm{NH}_{3}$ molecules was accomplished by Bethlem, et al. ${ }^{33}$ By directing a molecular beam through a series of electrode pairs which were switched at precise time intervals between high voltage and ground to create a moving potential well, a beam of $\mathrm{NH}_{3}$ was decelerated from $225 \mathrm{~m} / \mathrm{s}$ to $98 \mathrm{~m} / \mathrm{s}$. The apparatus used to decelerate the molecules is known as a Stark decelerator. Johannes Stark won the Nobel prize in 1919 for the discovery that atomic energy levels are split in the presence of an electric field. ${ }^{34}$ A Stark decelerator exploits the fact that polar molecules in certain quantum states will avoid regions of high electric field in order to minimize internal energy. These "low-field seeking" molecules are those in a quantum state which becomes higher in energy in the presence of an electric field.

The working principle of the decelerator is analogous to that of a linear accelerator. A low-field seeking molecule enters an array of electrode pairs which can produce an inhomogeneous electric field. As the molecule moves from a region of low field to a high field region, its internal energy is increased at the expense of translational energy. The molecule is 
slowed. Before the molecule passes between the electrode pair and regains its lost translational energy, the electrode is placed at ground voltage and the next pair downstream is energized. In this way, a molecule can be decelerated (or accelerated) in a stepwise fashion or even brought to a complete stop in a trap placed downstream from the decelerator. ${ }^{35-42}$

While the value of translationally cold molecules has been proven in the field of highprecision spectroscopy and cold molecules, a Stark decelerator has never been used to prepare molecules for surface scattering. With the goal of attaining complete control over both surface and scattering molecule, we designed and built a surface scattering apparatus capable of scattering carrier gas-free, quantum state-selected, Stark decelerated molecules from a surface that can be fully controlled and characterized in temperature, orientation, and atomic composition with great flexibility in experimental configuration, scattering molecule, and surface in order to elucidate energy transfer pathways with quantum state resolution.

The new apparatus consists of two modules. The first module produces a beam of velocity controlled, quantum state-selected molecules using a pulsed supersonic expansion of carbon monoxide (CO), followed by laser excitation to a chosen quantum state which allows for subsequent steering and velocity manipulation of the molecules using an electrostatic hexapole and a Stark decelerator. ${ }^{43}$ The thus-prepared beam is then scattered from a surface that is prepared and characterized in the second module. The surface module is equipped with equipment for cleaning and characterizing crystalline targets, decorating the crystals with welldefined coverages of adsorbates, measuring the surface work function and performing temperature programmed desorption (TPD) measurements. The region in which the molecular beam meets the surface allows laser access and features ion and electron detectors.

This thesis is organized as follows: in section 2, relevant background information and details of design and construction will be presented for the molecular beam and surface scattering module. Section 3.1 presents experimental results of thermal desorption experiments which were developed in order to create more complex surfaces from which to scatter. In section 3.2, experiments are presented in which electronically excited $\mathrm{CO}$ is scattered from clean and adsorbate covered $\mathrm{Au}(111)$ and de-excites, leading to the emission of electrons into vacuum. The results of the scattering studies illuminate the mechanism by which electronically excited $\mathrm{CO}$ quenches on a $\mathrm{Au}(111)$ surface. The work is summarized in section 4. 


\section{Background and Experimental}

\subsection{Velocity controlled molecular beams}

In order to scatter quantum state controlled molecules from microscopically characterized surfaces, we have designed and constructed a new molecule-surface scattering instrument. Presented in Figure 3 is a cutaway CAD model of the molecular beam line and surface scattering chamber of the machine. The apparatus consists of four differentially pumped vacuum chambers. The first three chambers comprise the molecular beam line and the fourth chamber is the UHV surface scattering chamber.

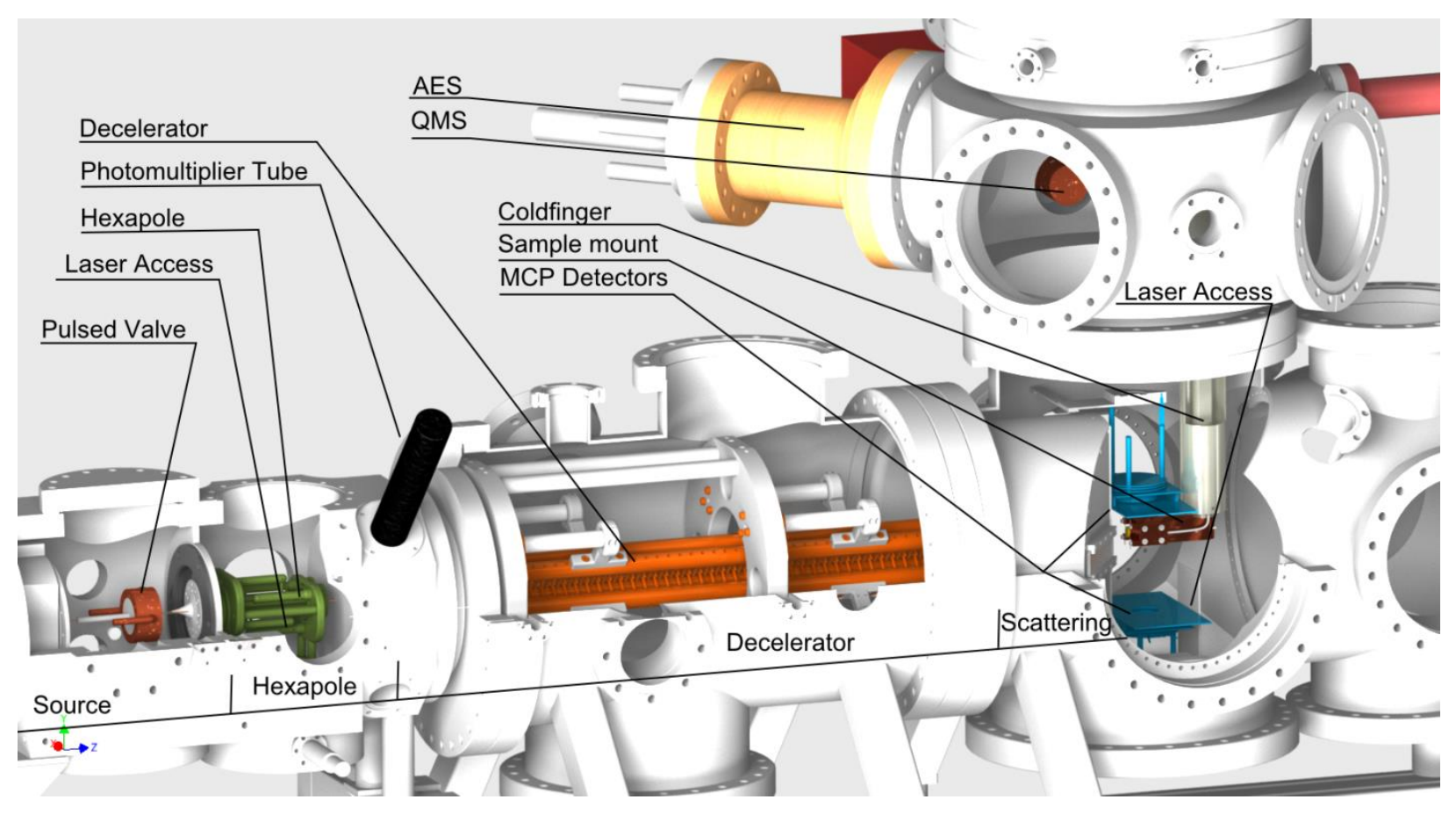

Figure 3 Cutaway drawing of surface scattering machine. AES: Auger electron spectrometer; QMS: quadrupole mass spectrometer; MCP: microchannel plate. The ticked line across the bottom of the figure identifies differential pumping regions. For more information, see text.

Starting from the left, the first chamber houses the molecular beam source, a cooled pulsed valve. The source chamber is connected via a sharp edged conical skimmer to the second differentially pumped chamber which houses an electrostatic hexapole. A third differential 
chamber is connected to the hexapole chamber via a $2 \mathrm{~mm}$ rounded aperture and houses a Stark decelerator. The fourth and final chamber is connected to the decelerator chamber via a $2 \mathrm{~mm}$ rounded aperture and comprises the UHV surface scattering chamber inside of which the sample holder, charged particle detectors, and all surface preparation and characterization equipment are mounted.

The following section presents background information and details of construction and performance of the new apparatus. The design of the surface-scattering device enables complete experimental control of incident molecule electronic, vibrational, rotational, and translational energies, as well as surface temperature, orientation, and composition.

\subsubsection{Production of carrier gas-free, state-selected molecular beams}

In order to perform a surface scattering experiment with full quantum state resolution, it is necessary to create a beam of molecules for which every degree of freedom can be controlled. All scattering experiments described in this work investigate the interaction between electronically excited carbon monoxide and a $\mathrm{Au}(111)$ surface. This section presents the techniques used to prepare a beam of quantum state-selected, carrier gas-free $\mathrm{CO}\left(\mathrm{a}^{3} \Pi_{1}\right)$, hereafter referred to as $\mathrm{CO}^{*}$, with a narrow distribution of velocities for scattering from the target surface.

The first electronically excited state of $\mathrm{CO}, \mathrm{a}^{3} \Pi$, has a large dipole moment of $1.37 \mathrm{D}$, with more electron density localized around the oxygen atom. ${ }^{44}$ Because decay from $a^{3} \Pi$ to the electronic grounds state is spin forbidden, the electronic state has a long radiative lifetime of 2.63 ms. ${ }^{39}$ The large dipole moment enables manipulation of $\mathrm{CO}^{*}$ with strong electric fields and the long lifetime makes the molecule suitable for molecular beam experiments in the laboratory.

\subsubsection{Molecular beam}

Molecular beams provide a useful tool for performing state resolved scattering experiments. Only a brief introduction to molecular beams will be given here. An excellent and thorough treatment can be found in Scoles. ${ }^{45} \mathrm{~A}$ molecular beam is produced by allowing a gas to leak isentropically through a small aperture from an area of high pressure to a low-pressure ambient background. As the gas flows through the aperture, there is a high rate of collisions, 
which leads to cooling of internal degrees of freedom as internal energy is converted into kinetic energy. If the pressure gradient is sufficiently high, the expansion will be supersonic. Supersonic expansions have a narrower final velocity and internal quantum state distribution than subsonic beams, known as effusive molecular beams. Pulsed beam sources produce a more intense beam than continuous beams while reducing the gas load in the expansion region.

Molecular beams allow the experimentalist to produce a narrow distribution of initial states in the beam and eliminates intermolecular collisions despite the high density of molecules. ${ }^{45}$ The absence of intermolecular collisions ensures that, aside from relaxation due to spontaneous emission, the molecule will remain in the chosen quantum state after excitation.

By seeding the molecule of interest in inert gases of varying masses, one can tune the final velocity distribution of the beam. Heavier gases expand out of the valve with lower velocity. The final velocity of the beam depends on the mass of seed gas and molecule and their relative concentrations and can be predicted using the formula:

$$
s_{\infty}=\sqrt{\frac{2 C_{p} T_{0}}{\bar{W}}}
$$

where $S_{\infty}$ is the final velocity, or terminal velocity, of the gas mixture, $C_{p}$ is the average molar heat capacity, $T_{0}$ is the temperature of the gas reservoir from which the mixture is leaked, and $\bar{W}$ is the average molar mass. ${ }^{45}$ From this equation, it can be seen that the terminal velocity of a molecular beam can be tuned by adjusting the carrier gas, nozzle temperature, and fraction of seed gas in the mixture. For pure hydrogen expanding from a room temperature reservoir equation 2.1 predicts $s_{\infty}=3000 \mathrm{~m} \mathrm{~s}^{-1}$. For pure xenon under the same conditions, equation 2.1 predicts $S_{\infty}=310 \mathrm{~m} \mathrm{~s}^{-1}$.

Seed gases, though inert, can stick for long periods of time on a cold surface. As the goal of state resolved molecule-surface scattering experiments is to develop a dynamical understanding of the interaction of molecules with surfaces, the composition of the surface must be well characterized during the scattering event. In order to avoid the problem of surface contamination by the molecular beam, our apparatus uses a hexapole filter to remove the carrier gas from a beam of excited CO molecules. Details can be found in section 2.1.1.3. 
Our apparatus employs a pulsed valve (Parker-Hannifin General Valve 99S4-A2-P299B08) which leaks short pulses of gas from a high pressure gas source (3 bar) into a low pressure ambient background $\left(10^{-6}\right.$ Torr with the beam running) at a $10 \mathrm{~Hz}$ repetition rate. The pulsed valve has been modified such that the tension on the spring controlling the force with which the poppet pushes against the exit aperture can be adjusted via an externally mounted micrometer. This adjustment makes the valve suitable for use over a broad range of temperatures. The valve temperature is controlled by flowing nitrogen gas through a copper coil submerged in a liquid nitrogen bath and subsequently through an inline resistance heater. Temperature of the valve is stabilized by referencing a PID controller (Eurotherm 2408) to a chromel/alumel (K-type) thermocouple mounted on the front face of the valve. The PID controller controls the current sent to the inline heater to produce a constant flow of cool nitrogen. Using this system, the temperature of the valve can be controlled within $\pm 0.5 \mathrm{~K}$ for twelve hours using $10 \mathrm{~L}$ of liquid nitrogen. Expanding 20\% CO seeded in argon or xenon through a valve cooled to 101 or $260 \mathrm{~K}$, respectively, produces a beam pulse with a most probable velocity of $360 \mathrm{~m} / \mathrm{s}$.

\subsubsection{Production of metastable CO}

The pulsed valve assembly is mounted on one of two specially designed flanges which can be reproducibly positioned on the source chamber using two precision machined alignment pins. One flange is used to mount the valve at $3.5^{\circ}$ relative to the main beam axis. Throughout the rest of this thesis mounting the valve in this orientation will be referred to as the bent geometry. The second flange mounts the pulsed valve on the main molecular beam axis (linear geometry). Each of the two flanges has a corresponding removable chamber wall which is installed downstream from the pulsed valve between the first and second differential pumping chambers. Both stainless steel plates are constructed with a mount for a molecular beam skimmer (beam dynamics, Model 2, Ni, $2 \mathrm{~mm}$ aperture), one in the bent orientation and the other in a linear geometry. Mounting the valve and skimmer in the bent geometry allows production of quantum state pure, carrier gas-free pulses of $\mathrm{CO}^{*}$ as described below. The surface scattering experiments presented in this work were performed with the valve in the bent geometry.

$\mathrm{CO}^{*}$ is produced in the molecular beam after passing through the skimmer by crossing it with a $206 \mathrm{~nm}$ laser pulse of approximately $10 \mathrm{~ns}$ duration and a bandwidth of $300 \mathrm{MHz}$ before 
entering a compact electrostatic hexapole focuser. The laser enters the vacuum chamber via 16 mm UHV fused silica laser windows mounted at Brewster's angle. The laser pulses are generated in a beta barium oxide (BBO) crystal by mixing the $4^{\text {th }}$ harmonic of a Nd:YAG (266 nm, Spectra Physics, Quanta-Ray Lab 170-10) pulsed laser with the output of a home built narrow bandwidth, all solid-state, injection seeded optical parametric oscillator (920 nm) which is pumped with the $2^{\text {nd }}$ harmonic of the same Nd:YAG laser $(532 \mathrm{~nm})$. Details of the construction and performance of this laser can be found in Velarde, et al. ${ }^{46} \mathrm{CO} *$ is prepared via the $\mathrm{a}^{3} \Pi_{1},(v=0, J=1) \leftarrow \mathrm{X}^{1} \Sigma^{+},(v=0, J=1)$ transition (Cameron band) at $206 \mathrm{~nm}$. Using a laser with suitably narrow wavelength, individual rotational states can be prepared.

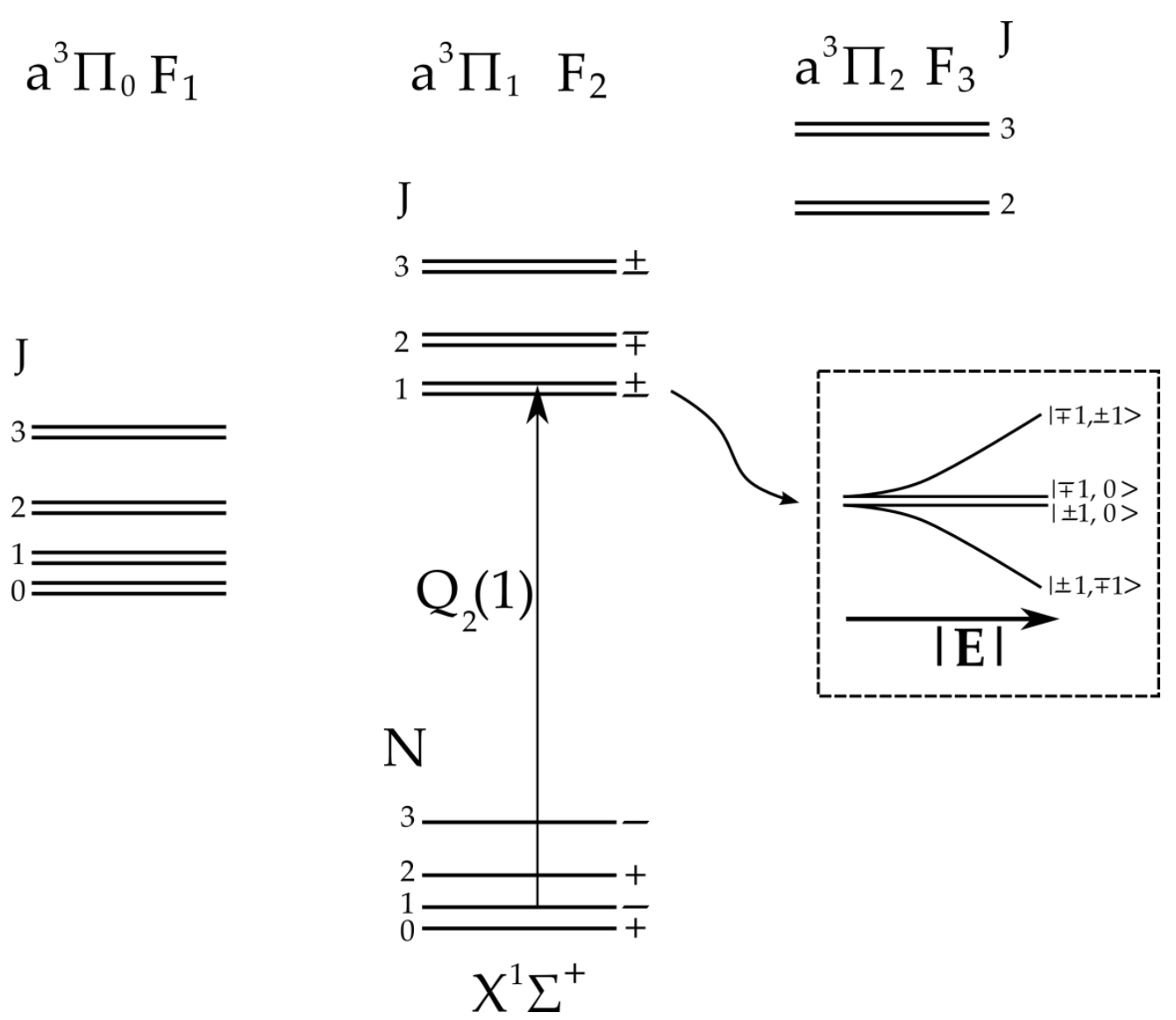

Figure 4 Scheme for excitation of $C O$ via the $a^{3} \Pi_{1} \leftarrow X^{1} \Sigma^{+}$transition. For low J-values, the most efficient transitions are to the $\Omega=1$ component of the $\mathrm{a}^{3} \Pi$ state, as shown. The behavior of the $\Lambda$-doublet components of the $a^{3} \Pi_{1},(J=1)$ state in an electric field are shown in the inset. 
A schematic of the excitation scheme is presented in Figure 4. The Hund's case (b) approximation best describes $\mathrm{CO}$ in the ${ }^{1} \Sigma^{+}$ground electron state. ${ }^{47}$ The rotational structure is fully characterized by the quantum number $N$, corresponding to the total angular momentum of the molecule minus electron spin. The parity of the rotational levels in the ground is given by $(-1)^{N}$. In the $\mathrm{a}^{3} \Pi$ state, the rotational structure is characterized by $J$, the total angular

momentum quantum number corresponding to the total angular momentum vector $\vec{J}$. For low values of $J$, the $\mathrm{CO}^{*}$ state is best approximated by Hund's case (a). Three different rotational ladders are distinguished in this approximation, corresponding to the possible values of the

quantum number $\Omega$, corresponding to $\vec{\Omega}$, the projection of the total angular momentum on the internuclear axis. Each $J$ level is split by $\Lambda$ doubling into two components with opposite parity as indicated in the inset of Figure 4.

The nominally spin forbidden $\mathrm{a}^{3} \Pi_{1},(v=0, J=1) \leftarrow \mathrm{X}^{1} \Sigma^{+},(v=0, J=1)$ transition is weakly allowed due to spin-orbit interaction of $\mathrm{a}^{3} \Pi$ state with the $A^{1} \Pi$ state. Therefore, the selection rules of the $A^{1} \Pi_{1} \leftarrow X^{1} \Sigma^{+}$are valid. For low $J$ states, considering a pure Hund's case (a) approximation, only transition to the $\Omega=1$ component is allowed. The $\mathrm{Q}_{2}(0)$ transition used in our experiment is indicated using the notation $\Delta J_{\Omega+1}\left(N^{\prime \prime}\right)$. Q indicates $\Delta J=0$.

The presence of a strong electric field lifts the $(2 J+1)$-fold degeneracy of the $M$ states, where $M$ is the projection of $\vec{J}$ in the direction of the electric field. In the $\mathrm{a}^{3} \Pi$ state, there is a first order Stark effect. ${ }^{44}$ The behavior of $\Lambda$-doublet components for the $J=1$ level of the $a^{3} \Pi_{1}$ state are shown in the inset of Figure 4. Those molecules in the $(\Omega, J, M)$ levels with a positive Stark effect, i.e. low-field seeking molecules whose internal energy increases with increasing field strength can be focused in an electrostatic hexapole and velocity-controlled using time-varying electric fields as will be shown in the following sections.

\subsubsection{Hexapole state selection}

The molecular beam is crossed with the excitation laser in the first part of the hexapole vacuum chamber. Immediately after excitation, the hexapole is placed at high voltage and focuses the $\mathrm{CO}^{*}$ by exploiting the Stark effect. Upon energizing the hexapole, those molecules in a low-field seeking state are driven to the electric field minimum in the center of the six rods in 
order to minimize their internal energy. The hexapole can be thought of as a lens for neutral dipolar molecules.

When the valve is mounted in the bent geometry, the beam pulse must be guided onto the decelerator axis in order to accelerate or decelerate the molecules. After the CO molecules have been electronically excited to $\mathrm{CO}^{*}$, hexapole voltage is switched on at $\pm 14 \mathrm{kV}$ and bends the $\mathrm{CO}^{*}$ molecules by $3.5^{\circ}$ through a $2 \mathrm{~mm}$ aperture into the third differential pumping chamber housing the Stark decelerator. The hexapole's unique design mounts all rods of like polarity onto one of two highly polished steel plates. The two 3-rod pieces are then mounted on individual MACOR ceramic supports opposite one another. Figure 5 shows a CAD model of the hexapole mounted on a DN100 Conflat ${ }^{\circledR}$ flange. The two stainless steel 3-rod pieces are represented in shades of blue, ceramic parts in white, and stainless steel in grey. The entire hexapole construction is mounted on an external micrometer-driven translation stage for in-situ adjustment of transverse hexapole transmission in order to optimize coupling of the molecular beam pulse into the decelerator.

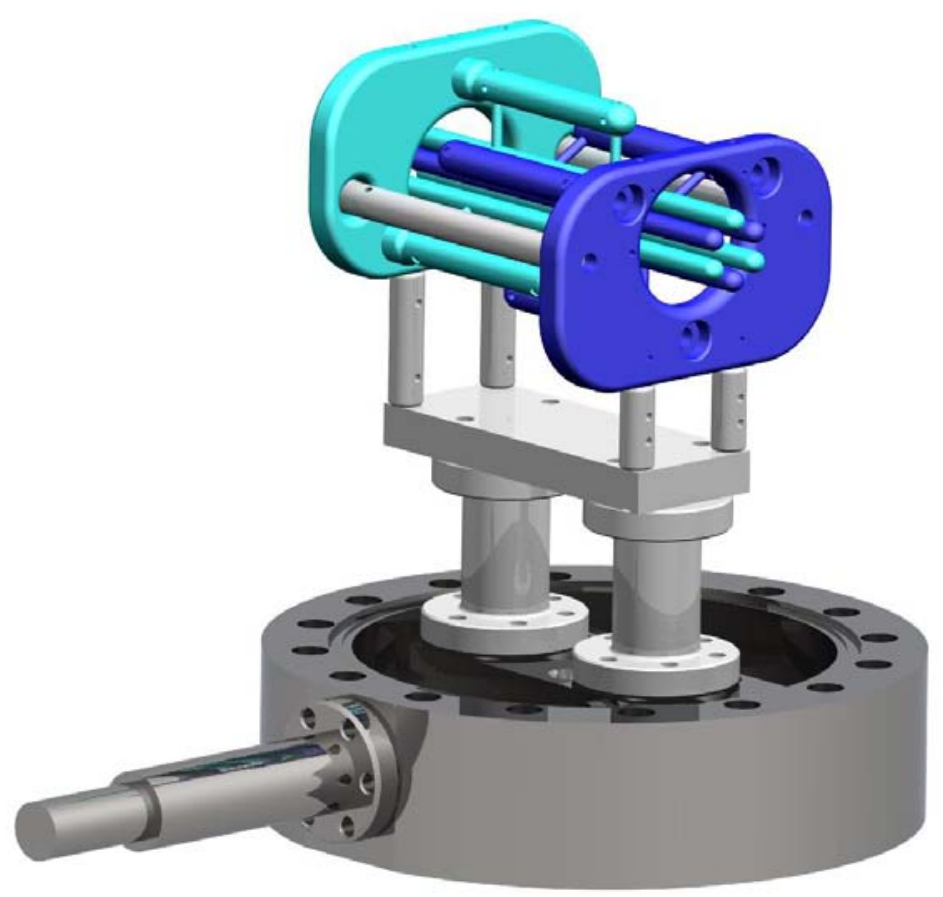

Figure 5 CAD model of hexapole mounted on CF 100 flange. In the drawing, MACOR pieces are white and the two stainless steel 3-rod pieces are shades of blue. The entire construction is mounted on a micrometer driven translation stage to optimize transverse position during operation. $^{48}$ 
This hexapole design has several advantages. Maximum operating voltage is limited by surface discharge via the insulator in which the electrodes are mounted. Using this design, the minimum surface distance (via the insulator mounts) between electrodes is $75 \mathrm{~mm}$, allowing operation at $\pm 14 \mathrm{kV}$ when switched at $10 \mathrm{~Hz}$. Using this operating voltage, the hexapole need only be $110 \mathrm{~mm}$ long to deflect a beam of $\mathrm{CO}^{*}$ with a mean velocity of $360 \mathrm{~m} / \mathrm{s}$ by the required $3.5^{\circ}$ through the $2 \mathrm{~mm}$ differential pumping aperture, as shown in the trajectory simulations in the left panel of Figure 6.
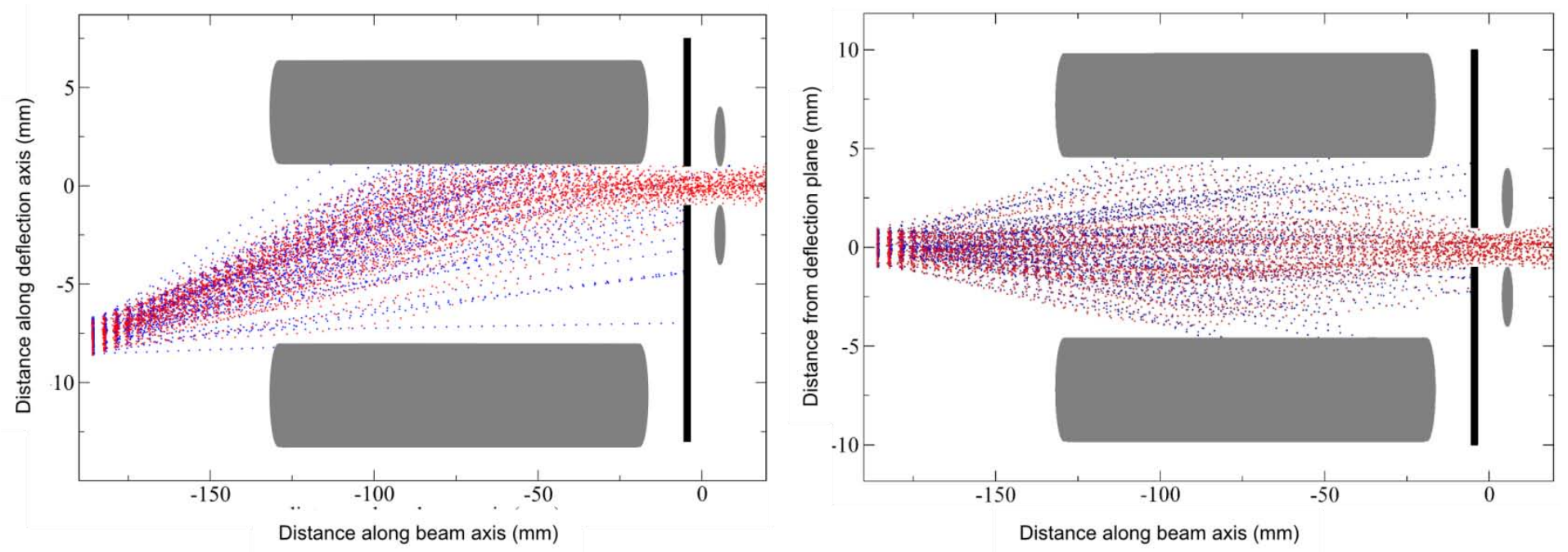

Figure 6 Trajectory simulations of CO* traveling at initial velocity of $360 \mathrm{~m} / \mathrm{s}$ through the hexapole with electrode voltages of $\pm 14 \mathrm{kV}$. The left panel represents hexapole mounted offcenter and molecular beam introduced into hexapole chamber at $3.5^{\circ}$ angle relative to the stark decelerator axis. The right panel depicts simulations of $\mathrm{CO}^{*}$ introduced on decelerator axis traveling at $360 \mathrm{~m} / \mathrm{s}$ through the hexapole at $\pm 14 \mathrm{kV}$ positioned on the center of the Stark decelerator axis. Both panels show the trajectory of the molecules on the horizontal plane (plane of deflection for left panel). In both panels, $\mathrm{CO}^{*}$ is represented by red dots and argon carrier gas by blue dots.

The left panel of Figure 6 shows a two dimensional trajectory simulation of CO* molecules introduced at $3.5^{\circ}$ relative to the beam axis passing through the hexapole along the plane of deflection with the hexapole offset from the axis of the molecular beam. The CO* molecules are represented as red dots and carrier gas as blue dots entering the hexapole at $3.5^{\circ}$ relative to the decelerator axis. The simulation clearly shows that nearly all of the electronically excited molecules are bent onto the decelerator axis and pass through the aperture into the next 
differential pumping region. The right panel shows a simulation using along the same plane, but with the $\mathrm{CO}^{*}$ molecules and carrier gas introduced on the axis of the molecular beam and the hexapole centered on the beam axis. Again, $\mathrm{CO}^{*}$ is represented by red dots and carrier dots by blue dots and nearly perfect transmission of $\mathrm{CO}^{*}$ into the next chamber is seen. Carrier gas transmission through the chamber is very inefficient, so the hexapole acts as a filter for $\mathrm{CO}^{*}$ molecules.

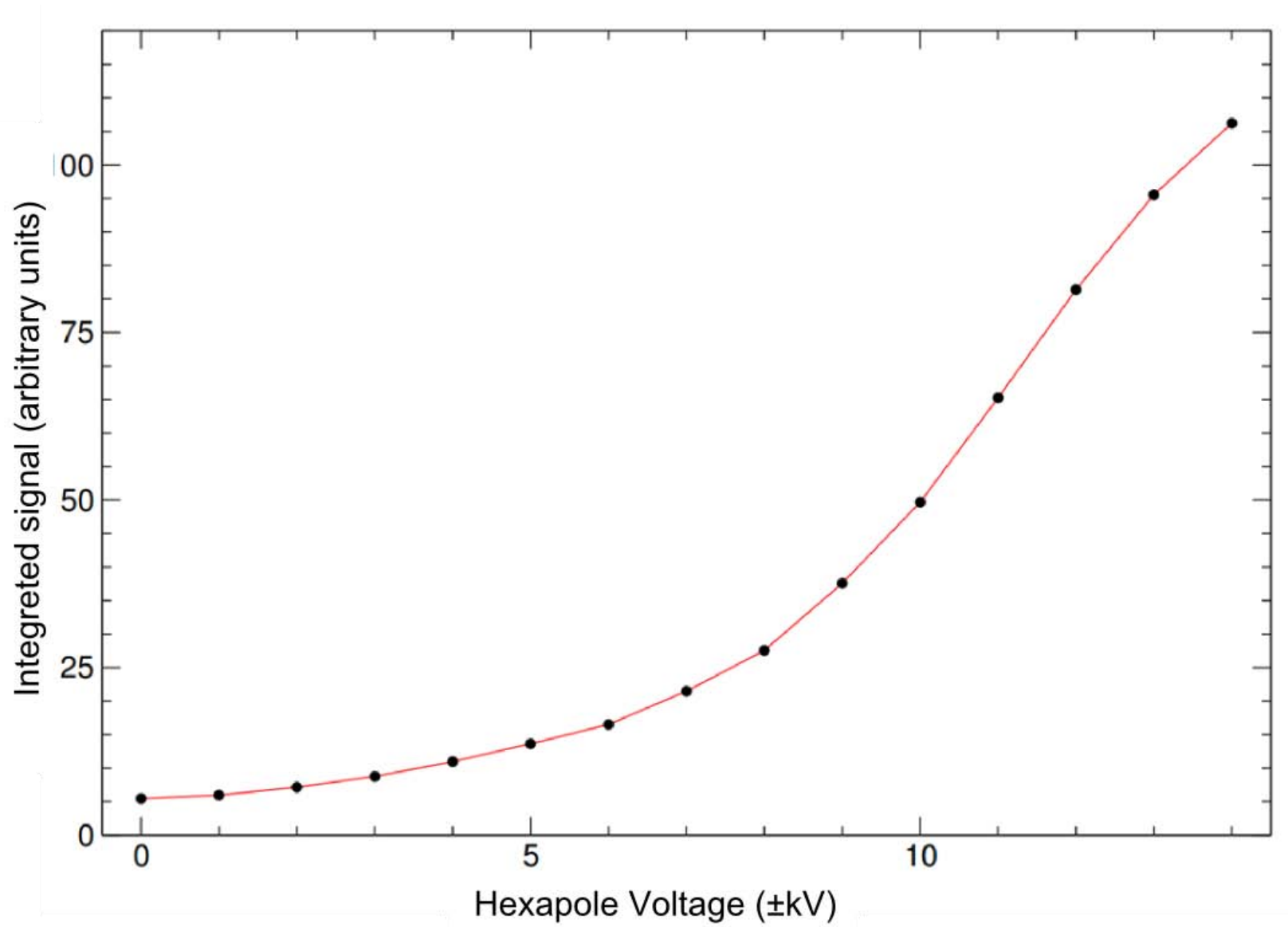

Figure 7 Integrated signal of $\mathrm{CO}^{*}$ molecules passing through the $2 \mathrm{~mm}$ aperture after hexapole as a function of applied voltage. Applied voltage was varied using constant optimized hexapole on/off switching times. The beam was detected by directing the $\mathrm{CO}^{*}$ at a microchannel plate (MCP).

Figure 7 shows the corresponding experiment. Here, I plot the integrated signal of CO* passing through the hexapole and subsequently through the $2 \mathrm{~mm}$ aperture in the differential pumping wall downstream at different hexapole voltages. Signal is recorded as the CO* 
molecules strike a microchannel plate (MCP) detector mounted in the next chamber. These measurements were made after optimizing hexapole on/off times and all measurements were made with these optimized switching times.

At initial beam velocities over $360 \mathrm{~m} / \mathrm{s}$, transmission through the hexapole chamber in the bent configuration begins to degrade as efficient deflection of the beam becomes impossible even at maximum voltage. However, when nozzle and skimmer are mounted in the linear configuration, the $\mathrm{CO}$ beam can be seeded in a light carrier gas to produce much faster beams. Although the hexapole is not capable of focusing a faster beam perfectly, even incomplete focusing will increase the coupling into the decelerator. This method, of course, will not result in a carrier gas-free beam, but the hexapole will ensure that transmission of $\mathrm{CO}^{*}$ through the hexapole is more efficient than that of the carrier gas. Also, the decelerator can be used to accelerate or decelerate the $\mathrm{CO}^{*}$ while leaving the carrier gas velocity unaffected and thereby separate the $\mathrm{CO}^{*}$ and the carrier gas into two different pulses. Thirdly, the decelerator's lateral bunching of $\mathrm{CO}^{*}$ with no comparable effect on the carrier gas will result in a well-defined, dense beam of $\mathrm{CO}^{*}$ with only a small fraction of the initial seed gas population entering the surface scattering chamber. This depletion of the seed gas is expected due to its transverse velocity through the hexapole and decelerator chambers and the fact that the beam pulse must pass through two $2 \mathrm{~mm}$ apertures between hexapole and surface.

After being focused into the third differential pumping chamber, the molecular beam pulse enters the Stark decelerator. Here, phosphorescence of the $\mathrm{CO}^{*}$ molecules is monitored using a solar blind photomultiplier tube (PMT, Hamamatsu R821, 160nm - 320 nm, gain: 4x10 ${ }^{5}$ ) mounted immediately downstream from the entrance to the decelerator chamber. The phosphorescence signal is used for optimization of experimental parameters such as laser wavelength and laser/molecular beam overlap.

\subsubsection{Stark deceleration}

The motion of neutral molecules in a beam can be manipulated using inhomogeneous electric fields and time-varying fields can be used to tune the velocity of beams of molecules to any arbitrary velocity. The following section will introduce the concepts involved in decelerating 
packets of neutral molecules using time-varying electric fields (Stark deceleration) and present details of construction and performance of a new decelerator that was built to perform the first molecule-surface scattering experiments using Stark-decelerated molecular beams.

\subsubsection{Principles of Stark deceleration}

Stark deceleration is the process of manipulating the velocity of neutral polar molecules using time-varying strong inhomogeneous electric fields and has been previously reviewed in detail in several publications. ${ }^{35,38,40,43}$ The following section will first present the basic concepts of Stark deceleration, then provide construction details of the decelerator used in the present report which has been designed to survive bake-out cycles of $100{ }^{\circ} \mathrm{C}$ for $36-48$ hours without becoming misaligned, and finally present results showing the versatility of this decelerator in tuning the velocity of $\mathrm{CO}^{*}$ molecules.

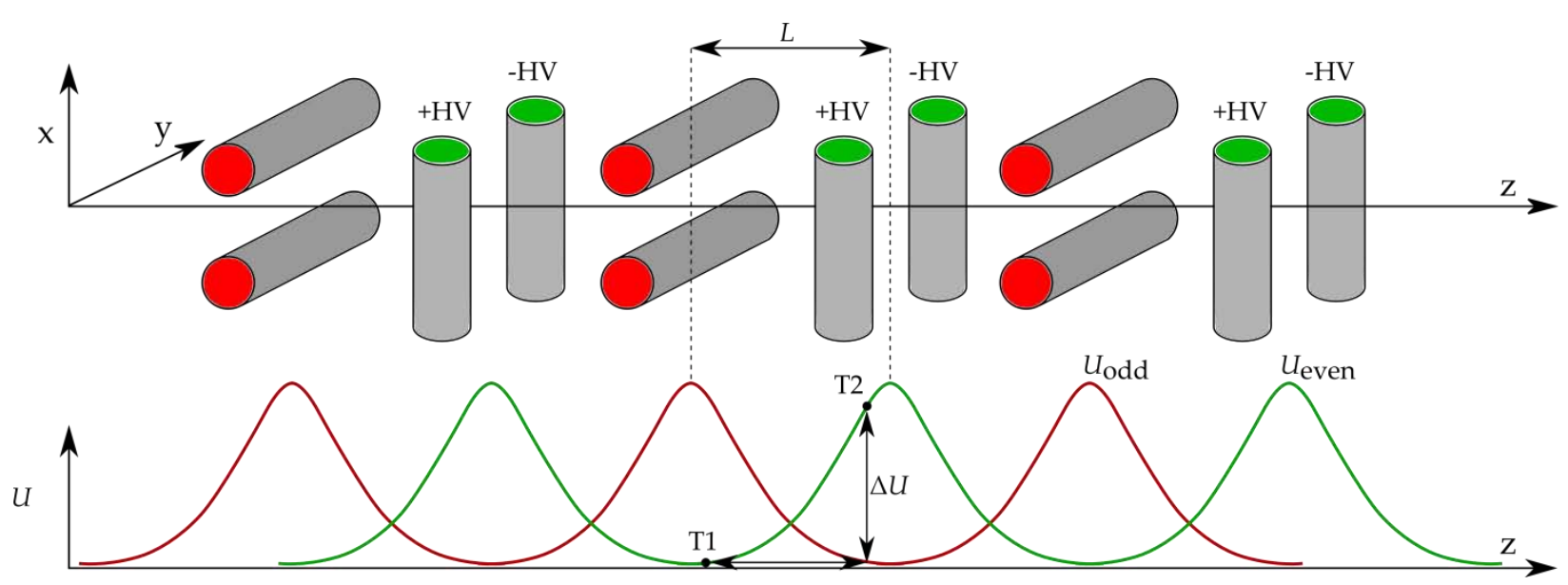

Figure 8 Schematic representation of the potential energy changes experienced by a low-field seeking molecule traveling through a Stark decelerator. Electrode pairs of like orientation are positioned with a center-to-center distance of $2 L=11 \mathrm{~mm}$. As the molecule travels along the decelerator axis, $\mathrm{z}$, the decelerator is switched between $\mathrm{U}_{\text {odd }}$, with odd (red-capped) electrodes at high voltage and $U_{\text {even }}$ with even (green capped) electrodes at high voltage. While in the $U_{\text {odd }}$ $\left(U_{\text {even }}\right)$ configuration, all even (odd) electrode pairs are at ground potential. $\Delta U$ is the change in the molecule's internal energy when switching from $U_{\text {even }}$ to $U_{\text {odd. }}$ Low-field seeking molecules can be accelerated or decelerated to any arbitrary velocity by changing the timings with which the decelerator is switched to add or remove Stark energy, $U$, from the molecule at the expense of translational energy. See text for more information.

Figure 8 shows the change in internal energy experienced by a low-field seeking molecule due to the Stark effect as it travels through the decelerator. The basic design of the 
decelerator is also clear from Figure 8. Parallel pairs of cylindrical metal rods are organized into a periodic array with electrode pairs alternating between vertical and horizontal orientation. All electrodes of like orientation are mounted to one of four metal rods which can be placed under high voltage. When an electrode pair is under high voltage, the magnitude of the voltage is the same for each electrode, but it is of opposite polarity.

The decelerator can be switched between two different configurations. In one configuration electrodes of the first, third, and all odd numbered (horizontal) electrodes are placed under high voltage while even numbered (vertical) electrodes remain at ground potential. The change in internal energy of a molecule traveling through the decelerator while in this configuration is represented by the red curve, $U_{\text {odd }}$. In the second configuration, odd electrode pairs are kept at ground potential while even electrode pairs are placed at high voltage. The second configuration results in the molecule experiencing a change in internal energy represented by $U_{\text {even }}$.

The decelerator can be operated in one of three modes: deceleration, acceleration, or guiding. First we will examine deceleration mode. As seen in Figure 8, a molecule flying along the decelerator axis, $\mathrm{z}$, with the decelerator in the $U_{\text {even }}$ configuration beginning at time T1 approaches a field maximum. As it moves toward the region of higher electric field, its Stark energy increases at the expense of translational energy. The molecule is decelerated. If the molecule continues flying in the same direction past the charged electrodes, it will regain its lost kinetic energy as leaves the electric field. If, however, the decelerator is switched from the $U_{\text {even }}$ to the $\mathrm{U}_{\text {odd }}$ configuration at time $\mathrm{T} 2$, the molecules loses the kinetic energy permanently and "sees" another field maximum directly downstream and the process is repeated.

By switching the decelerator between configurations at the appropriate times, a chosen amount of kinetic energy can be removed from the molecule at each stage. Using the example of a molecule beginning at time T1, the amount of Stark energy gained by the molecule at each stage, $\Delta U$, can be controlled by varying time T2. In this way the final velocity of the molecule leaving the decelerator can be selected. For a quantitative description of the molecules' trajectories in the decelerator, terminology is borrowed from accelerator physics. 
The magnitude of translational energy removed from the molecule depends on the position of the molecule relative to the electrodes when the fields are switched. The phase angle is defined as $\phi=2 \pi\left(\frac{z}{2 L}\right)$ where $z$ is the molecule's position along the beam in units of $L$. Switching the fields at the electric field maximum corresponds to a phase angle of $\phi=90^{\circ}{ }^{35,48}$

By timing the switches such that the electrodes are placed at high voltage after the molecule has flown past, the $\mathrm{CO}^{*}$ can also be accelerated in an analogous fashion. These timing schemes are designated with negative phase angles. An additional advantage of the construction of our decelerator is that as the molecules fly toward each charged electrode pair, the field minimum is at the midpoint between the two electrodes. There is a force driving low-field seeking molecules toward the molecular beam axis and the beam pulse is spatially focused in one or the other transverse direction at each deceleration stage. This transverse focusing is necessary as the distance from pulsed valve to surface is $96 \mathrm{~cm}$.

In the previous discussion, it has been assumed that the decelerator will switch configuration every time the molecule passes an electrode stage. This mode of operation is known as $s=1$ mode. The decelerator can also be switched as the beam pulse passes any odd number of stages $(s=3,5, \ldots)$. For example, in $s=3$ mode, the particle travels between one charged electrode pair and is focused toward the field minimum along the decelerator axis, then between an uncharged electrode pair, and the decelerator is then switched at some phase angle relative to the third electrode pair.

The decelerator can also be operated in guiding mode (phase angle of $\phi=0^{\circ}$ ), which does not change the velocity of the molecules, but solely takes advantage of the transverse focusing properties of the decelerator. This mode of operation merely guides the molecular beam through the decelerator at its initial mean velocity. Switching between $U_{\text {even }}$ and $U_{\text {odd }}$ in $s=3$ takes advantage of transverse focusing and in this way the density of the molecular beam pulse can be preserved as the beam passes through the decelerator chamber and into the surface scattering chamber.

When discussing the longitudinal motion of a packet of molecules through the decelerator, it is necessary to introduce the concept of "phase stability." Phase stability was discovered independently by Veksler ${ }^{49}$ and McMillan $^{50}$ and forms the basis of modern particle 
accelerators. Phase stability is what allows the manipulation of packets of molecules with a distribution of starting positions and velocities. In order to discuss phase stability, on must first introduce phase space. A phase space diagram consists of 2 phase space variables for each direction (position and momentum) with time as the dependent variable. Often, the coordinates are described in terms of "error" relative to the synchronous molecule, defined below. See Figure 9 for a representative phase space plot.

In order to clarify phase stability, let us first consider the case of guiding mode in $\mathrm{s}=1$ where the fields are switched at a constant $\Delta T$ and a molecule at phase $\phi=0^{\circ}$ with a velocity that exactly matches the frequency of the electric field switching times. That is to say, the molecule travels exactly the distance $L$ in the time interval $\Delta T$. This molecule is referred to as the synchronous molecule. The synchronous molecule is always in phase. ${ }^{35}$ The synchronous molecule's phase and velocity are referred to as the equilibrium phase, $\phi_{0}$, and equilibrium velocity, $v_{0}$. Two things are apparent in this scenario. First, the phase and velocity of the molecule remain unchanged. Second, molecules with a different phase or velocity will automatically correct toward these equilibrium values.

A molecule with the same velocity as the synchronous molecule but with phase slightly higher than $\phi_{0}$ will be farther along the decelerator axis when the fields are switched and will be slightly decelerated relative the synchronous molecule at every electrode stage. Its phase will get smaller until it lags behind the synchronous molecule. Thereafter, it will be slightly accelerated relative to the synchronous molecule and so on. This shows that molecules with phase $\neq \phi_{0}$ will oscillate in velocity and phase around the equilibrium values as long as the restoring force is high enough. Synchronous and slightly asynchronous molecules can be thought of as trapped in a moving potential well travelling at the velocity of the synchronous molecule. See Figure 9. 

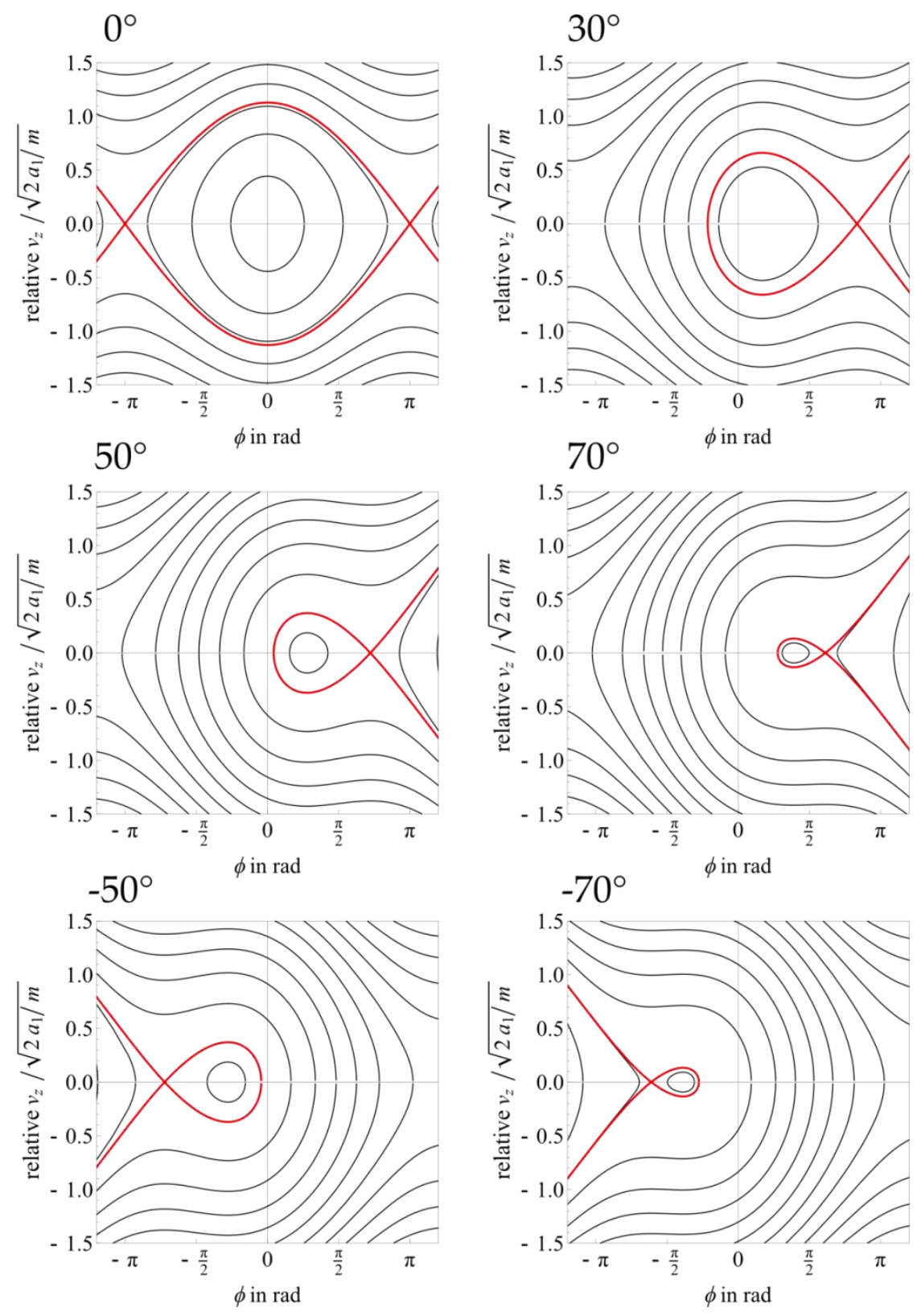

Figure 9 Phase space of longitudinal motion for different phase angles, $\phi_{0}$. The thick red lines indicate the separatrices. Notice that with increasing phase angle, the size of the phase-stable region decreases (see text). Energy difference between trajectories is $0.5 a_{1}$ for all phase angles. ${ }^{48}$

In the case of deceleration (acceleration) the synchronous molecule still travels the distance $L$ during the time interval $\Delta T$, but $\phi_{0} \neq 0^{\circ}$. Since the translational energy of the synchronous molecule is reduced (increased) at every electrode stage, $\Delta T$ grows longer (shorter) at each stage. The synchronous molecule will therefore always be at the same position every time 
the fields are switched ( $\phi_{0}$ remains constant). The behavior of molecules with nonsynchronous phase and velocity will oscillate around the synchronous molecule in exactly the same way as in guiding mode.

The amount of translational energy lost by the synchronous molecule per deceleration stage, $\Delta K\left(\phi_{0}\right)$, is equal to the Stark energy the molecule has gained before the fields have been switched: $U\left(\phi_{0}\right)-U\left(\phi_{0}+\pi\right) . U(\phi)$ can be expressed as a Fourier series. In the expression for $\Delta K\left(\phi_{0}\right)$, all even terms cancel, yielding:

$$
\Delta K\left(\phi_{0}\right)=2 a_{1} \sin \left(\phi_{0}\right)+2 a_{3} \sin \left(3 \phi_{0}\right)+\cdots
$$

When electrode stages are not too far apart, as in the decelerator used in this work, $\Delta K\left(\phi_{0}\right)$ is determined to a good first approximation by the first term. Phase is, according to the definition above, defined only at the moment when fields are switched. A description of terms in continuous variables is needed to describe the motion of molecules through the decelerator. The instantaneous difference in phase, $\Delta \phi=\phi_{\mathrm{n}}-\phi_{0}$, and velocity, $\Delta v=v_{\mathrm{n}}-v_{0}$, for a nonsynchronous molecule are introduced in order to describe the motion of the nonsynchronous molecule through the decelerator, where the subscript $n$ denotes the phase or momentum of the nonsynchronous molecule. The kinetic energy lost by the synchronous molecule at each stage can be regarded as originating from a continuously acting average force:

$$
\bar{F}\left(\phi_{0}\right)=-\Delta K\left(\phi_{0}\right) / L
$$

This approximation is valid when the velocity reduction, $\Delta v$, of the synchronous molecule at each stage is small relative to $v_{0}$. When $\Delta v \ll v_{0}$, the average force on the nonsynchronous molecule can be written as:

$$
\bar{F}\left(\phi_{0}+\Delta \phi\right) \cong-\Delta K\left(\phi_{0}+\Delta \phi\right) / L
$$

The force acting on that molecule relative to the synchronous molecule for a fixed phase angle can be expressed by:

$$
F_{\phi_{0}}(\Delta \phi) \cong-\frac{2 a_{1}}{L}\left[\sin \left(\phi_{0}+\Delta \phi\right)-\sin \left(\phi_{0}\right)\right.
$$


and motion of the nonsynchronous molecule can be described relative to that of the synchronous molecule:

$$
\frac{m L}{\pi} \frac{d^{2} \Delta \phi}{d t^{2}}+\frac{2 a_{1}}{L}\left[\sin \left(\phi_{0}+\Delta \phi\right)-\sin \left(\phi_{0}\right)\right]=0
$$

where $m$ is the mass of the molecule. This is analogous to the equation for a biased pendulum, i.e. a pendulum driven by a constant torque.

The potential for effective force can be derived from equation 2.4, as shown in Friedrich, et al. ${ }^{51}$ :

$$
V_{\phi_{0}}(\Delta \phi) \cong-\frac{2 a_{1}}{\pi}\left[\cos \left(\phi_{0}+\Delta \phi\right)+\left(\phi_{0}+\Delta \phi\right) \sin \left(\phi_{0}\right]\right.
$$

Much can be learned about the stability of longitudinal motion through the decelerator by plotting the longitudinal phase space. These plots are the curves of velocity, $v(\phi)$ versus longitudinal position for different total energies at fixed phase angles. $v$ and $\phi$ are related via $v=\frac{\mathrm{d} z}{\mathrm{~d} t}=\frac{L}{\pi} \frac{\mathrm{d} \phi}{\mathrm{d} t}$. One important feature of these phase space plots is the magnitude $V_{\max }=$ $V_{\phi_{0}}\left(\phi_{\max }\right)$ at the position $\phi_{\max }$ of the local maxima of $V_{\phi_{0}}$. If a molecule's total energy is less than $V_{\text {max }}$, it will be confined in a potential well and oscillate around the synchronous molecule. If the molecule's energy is greater than $V_{\max }$, the molecule will leave the traveling potential well centered on the synchronous molecule and will not be transmitted through the decelerator as intended.

Of course, under experimental conditions, most of the molecules entering the decelerator will not be perfectly in phase. The range of positions and velocities which can be successfully transmitted through the decelerator (phase space acceptance) is determined by the phase-angle of the synchronous molecule. For deceleration experiments, phase angle is set to $0<\phi_{0}<90^{\circ}$, where larger phase angle corresponds to more translational energy removed from the synchronous molecule per stage. ${ }^{43}$ As phase angle increases, however, phase space acceptance decreases. 
This phenomenon is shown in Figure 9 for several different values of $\phi_{0}$. The thick red line is known as a separatrix. Those molecules whose total energy places them within the separatrix are said to be phase-stable, meaning that the phase of the molecule oscillates within certain bounds. This concept underscores the basic principle of a Stark decelerator in which molecules are stably confined within a certain phase space.

Shown in Table 1 are velocities and associated kinetic collision energies, $v_{\mathrm{f}}$ and $E_{\text {coll }}$, and associated half-widths, $\Delta v_{\mathrm{f}}$ and $\Delta E_{\text {coll }}$ calculated using three dimensional trajectory simulations corresponding to different phase angles. ${ }^{48}$

Table 1 Calculated final velocities, $v_{\mathrm{f}}$, longitudinal velocity spread, $\Delta v_{\mathrm{f}}$, collision kinetic energy, $E_{\text {coll }}$, and collision energy spread, $\Delta E_{\text {coll }}$ for initial beam velocities of $360 \mathrm{~m} / \mathrm{s}$. ${ }^{48}$

\begin{tabular}{|c|c|c|c|c|}
\hline$\varphi_{0}\left({ }^{\circ}\right)$ & $v_{\mathrm{f}}(\mathrm{m} / \mathrm{s})$ & $\Delta v_{\mathrm{f}}(\mathrm{m} / \mathrm{s})$ & $E_{\mathrm{coll}}\left(\mathrm{cm}^{-1}\right)$ & $\Delta E_{\mathrm{coll}}\left(\mathrm{cm}^{-1}\right)$ \\
\hline-90 & 512 & 4 & 307 & 4.8 \\
-50 & 470 & 11 & 259 & 12.1 \\
-30 & 430 & 16 & 216 & 16.1 \\
0 & 360 & 14 & 152 & 11.9 \\
30 & 272 & 16 & 87 & 10.2 \\
40 & 235 & 14 & 65 & 7.4 \\
50 & 193 & 11 & 44 & 5.0 \\
60 & 145 & 8 & 25 & 2.7 \\
70 & 87 & 6 & 8.9 & 1.2 \\
75 & 50 & 4 & 3.0 & 0.5 \\
\hline
\end{tabular}

As a packet of molecules travels through the decelerator, the distribution of molecules in phase space can be modeled very accurately using three dimensional trajectory models. The simulations reported in this work were performed by Grätz ${ }^{48}$ and resulted in highly accurate predictions of the phase space distributions of molecules leaving the decelerator, as shown in Figure 10. The distinctive spiral shape (in phase space) which the pulse develops results from different oscillation frequencies of the coupled molecules. This results in rotation in phase space 
of the packet of $\mathrm{CO}^{*}$ molecules in the region bound by the separatrix and leads to the characteristic shape of the time-of-flight peak.
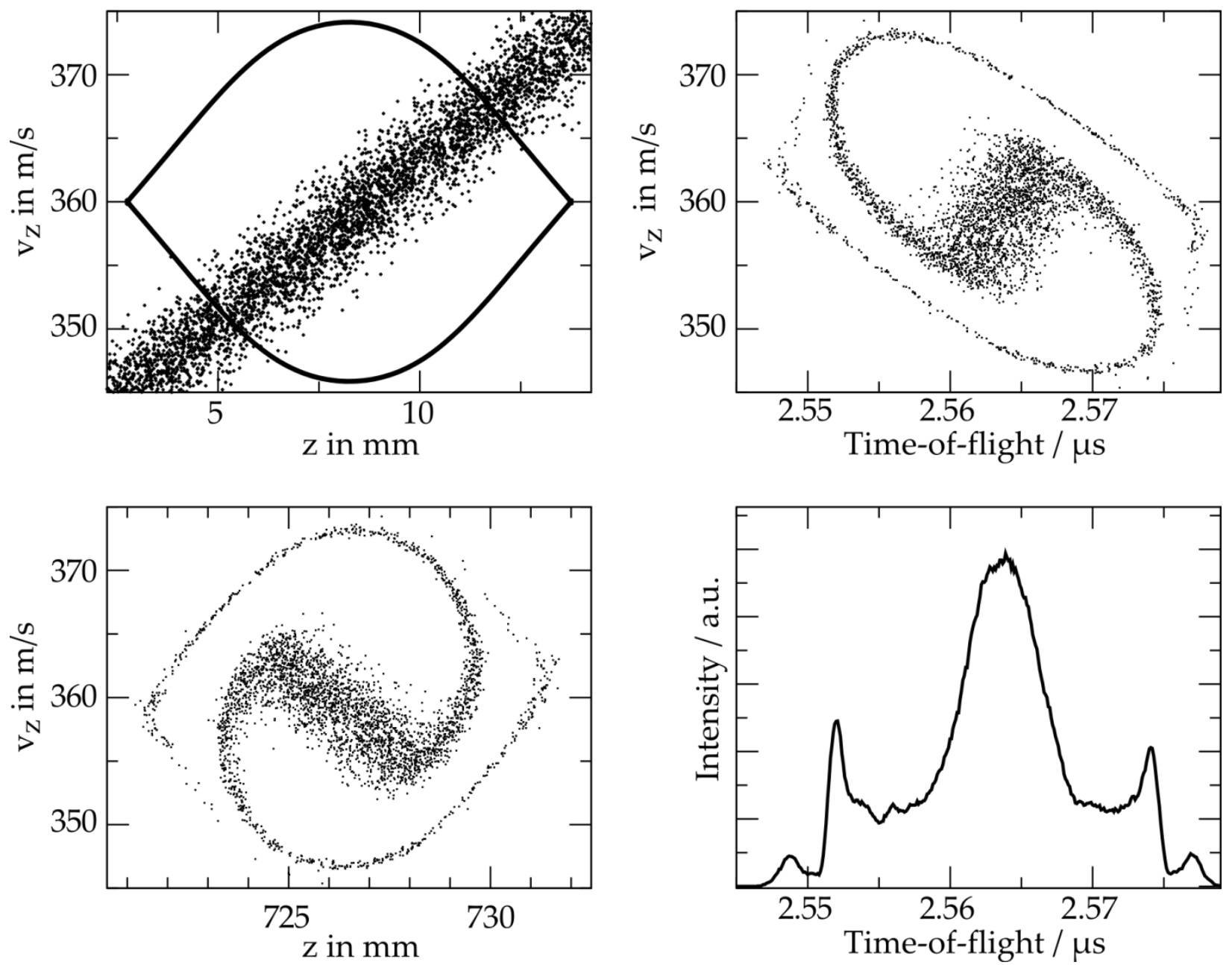

Figure 10 Upper left panel: a narrow slice of the molecular beam couples into the Stark decelerator after leaving the hexapole. Lower left panel: coupled molecules rotate in phase space as they travel through the decelerator. Upper right panel: The characteristic spiral structure of the beam pulse broadens in time and space after leaving the decelerator and collides with the surface after $23 \mathrm{~mm}$ free flight. Lower right panel: Resulting electron emission signal corresponds to simulations almost perfectly. 


\subsubsection{Construction of a bakeable Stark decelerator}

The decelerator is made up of 131 deceleration stages comprised of 132 equidistant pairs of parallel $3 \mathrm{~mm}$ diameter cylindrical electrodes with hemispherical caps. The distance between paired electrodes is $5 \mathrm{~mm}$, center to center. Each pair is spaced $L=5.5 \mathrm{~mm}$ apart, center to center, and positioned perpendicular to the pair on either side. Each electrode is very carefully polished to allow for fast switching between either $16.5 \mathrm{kV}$ or $-16.5 \mathrm{kV}$ and ground with no electric discharge.

In order to the achieve the UHV conditions necessary for surface scattering experiments we bake the surface and decelerator chambers to around $100^{\circ} \mathrm{C}$ for $24-48$ hours. Therefore, the decelerator described here differs from previous successful decelerator designs such that it can survive bake-out conditions. As shown in Figure 11, each stainless steel rod to which the electrodes are attached is connected via two ceramic rods to a polished stainless steel support frame. Our design mounts the decelerator using ceramic rods that all point in the same direction. Earlier versions mounted the 4 ceramic rods on either side pointing in opposite directions. Using the new design, heating the decelerator does not lead to severe mechanical stress despite the difference in coefficient of linear thermal expansion of MACOR ceramic and stainless steel. Baking results in a movement of the decelerator with respect to the frame, but each of the contact points move the same distance in the same direction and the crucial alignment of the decelerator is preserved. This simple modification allows us to safely bake the decelerator up to $100^{\circ} \mathrm{C}$. The decelerator has been tested through 20 bake-out cycles with no loss of signal, indicating that alignment is not affected. 


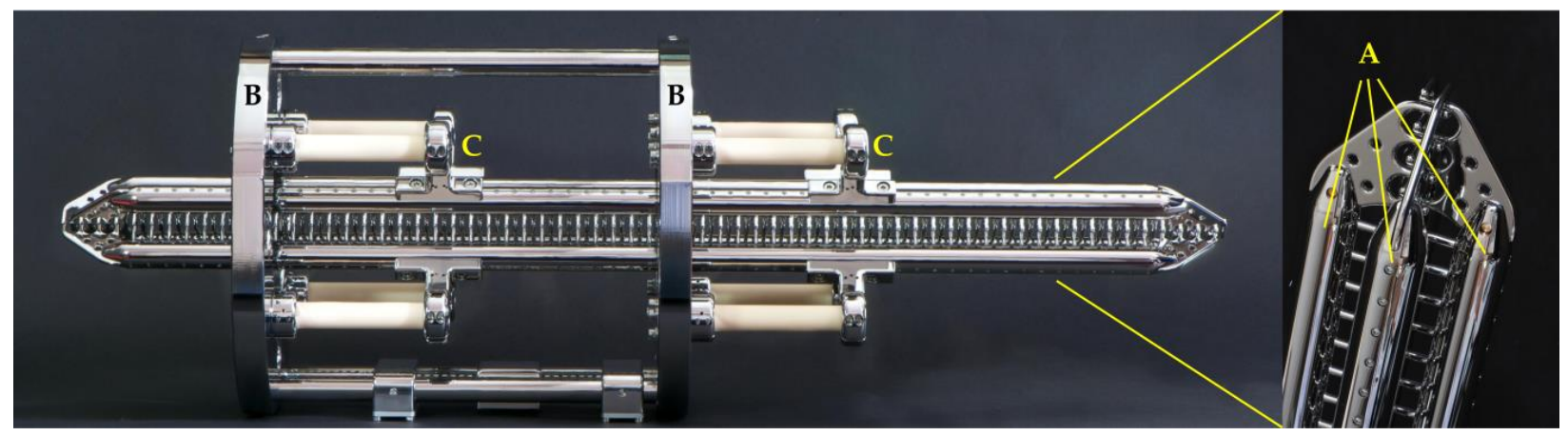

Figure 11 The left panel shows the fully bakeable Stark decelerator. Each of four stainless steel mounting rods (A) is affixed to two mounting plates (B) via two MACOR rods (C) mounted in the same orientation, allowing the device to be baked at $100{ }^{\circ} \mathrm{C}$ without inducing undue mechanical stress. The right panel shows a close-up of the perpindicular electrode pairs constructed by mounting four stainless steel mounting rods and specially designed end pieces which allow for a very short distance from the last decelerator electrode pair to the surface scattering chamber. Photographs by Georg Heyne, FHI Berlin.

As will be described in section 2.2.4.2, laser access to the decelerator has been achieved by mounting a special end cap on the surface side of the decelerator chamber which extends into the surface chamber. Two O-ring sealed UV fused silica windows are installed at Brewster's angle on either side of the cap to allow laser access to the molecular beam for six electrode stages before the end of the decelerator. This allows us to laser prepare molecules which have travelled through the decelerator. One envisioned experiment is to use stimulated emission pumping to populate a chosen ro-vibrational level in the electronic ground state before the pulse has left the decelerator. Then, according to simulations, by over-focusing with the last decelerator stages we can deflect the remaining metastables away from the beam axis. In this way, a beam of pure electronic ground state molecules can be prepared for scattering from the surface. Other spectroscopic preparation schemes will be discussed in the relevant results section. 


\subsection{Surface preparation and scattering}

The previous section described the capability of our new apparatus to prepare a beam of quantum state-selected molecules with an extremely narrow velocity distribution. The goal of our research is to elucidate the energy transfer processes which occur at the gas-surface interface. The following section describes the heart of the experiment: the UHV surface scattering chamber.

First, the preparation of an atomically clean $\mathrm{Au}(111)$ crystal surface will be described. Then, the techniques used to prepare more complex surfaces with well-defined adsorbate coverages will be presented. In order to understand the complex surfaces, both the composition and the electronic properties must be examined as a function of adsorbate coverage. Precise adsorbate coverage information is achieved using temperature programmed desorption (TPD). TPD not only yields information about the composition of the surface, but also yields useful kinetic information about the adsorbate-substrate system. The electronic work function of clean and adsorbate decorated surfaces is measured with a Kelvin probe. Finally, the experimental details of the surface scattering experiments will be presented. The incoming molecular beam pulse is characterized via detection of phosphorescent photons as $\mathrm{CO}^{*}$ spontaneously decays to the electronic ground state. The incident beam pulse is also characterized via resonance enhanced multiphoton ionization (REMPI). As the beam of $\mathrm{CO}^{*}$ molecules impinges on the $\mathrm{Au}(111)$ surfaces, the molecule is quenched to the ground electronic state and electrons are emitted. The experimental details of electron detection are presented as the final part of this section. 


\subsubsection{Atomically clean Au(111) surface}

After the molecular beam exits the decelerator, it passes through a $2 \mathrm{~mm}$ rounded aperture into the UHV surface chamber where it is scattered from the surface. The surface chamber consists of two tiers. The upper level houses all surface characterization and preparation equipment including an ion-sputtering gun (STAIB INSTRUMENTS, IG-5-C), Auger electron spectrometer (STAIB INSTRUMENTS, ESA-150), gas manifold for deposition of adsorbate gas onto the surface, residual gas analyzer (SRS RGA-200) for leak detection and thermal desorption measurements and Kelvin probe (KP Technology Ltd. UHV-KP010USB) for work function measurements. Surface scattering take place in the lower level, where MCP detectors (MCP, tectra GmbH Physikalische Instrumente MCP 050, 40mm, chevron configuration), ion optics, and laser access are mounted.

\subsubsection{Vacuum considerations}

The foremost requisite for a surface scattering experiment is that the scattering process of interest occurs under conditions of ultra-high vacuum (UHV). Vacuum is integral to both fundamental parts of the experiment: the molecule and the surface. Preserving the quantum states prepared in the molecular beam for any useful amount of time is only possible in the absence of collisions between the molecule of interest and anything other than the surface. Preventing the molecules from colliding with each other and the walls of the vacuum chamber is achieved by using a supersonic expansion in the beam source and collimation, as described above. In order to prevent collisions with other molecules present in the vacuum chamber, it is necessary to place the entire vessel, from source to detector, under vacuum. Additionally, any gas which impinges on the sample surface has a chance to physisorb or chemisorb and change the topography and physical properties of the surface. Any unintended pollution of the surface can result in experimental results which cannot be accurately interpreted due to lack of knowledge of the experimental system. As will be shown below, it is the surface which imposes the most stringent limitations on acceptable pressure in the scattering chamber.

When discussing vacuum, many helpful concepts can be derived from the kinetic theory of gases to help illustrate the immense change in freedom of movement experienced by a molecule of gas with decreasing pressure. A more exhaustive treatment may be found in any 
textbook on the kinetic theory of gases. ${ }^{52}$ A molecule's average velocity, $\bar{v}$, can be deduced from the Maxwell-Boltzmann velocity distribution law:

$$
\bar{v}=\sqrt{\frac{8 k T}{\pi m}}
$$

Where $m$ is the mass of the particle, $k$ is the Boltzmann constant, and $T$ is the temperature of the gas. Intermolecular collision frequency, $Z$, mean free path, $\lambda$, and frequency of collisions per unit time, $Z_{S}$, can be calculated using velocity, $\bar{v}$, molecular diameter, $\xi$, and number density, $n$. $\xi$ for typical diatomics such as $\mathrm{N}_{2}, \mathrm{O}_{2}$, or $\mathrm{CO}$ around $3 \times 10^{-10} \mathrm{~m}$. Number density can be calculated directly from pressure.

$$
\begin{gathered}
Z=\sqrt{2} \pi \xi^{2} v, \\
\lambda=\frac{1}{\sqrt{2} n \pi \xi^{2}} \\
Z_{S}=\frac{n v}{4}
\end{gathered}
$$

Table 2 shows these values calculated as a function of pressure. Monolayer formation time assumes a sticking coefficient of 1 . It should be noted that monolayer sticking time is a lower limit. A sticking coefficient of 1 for chemically inactive species present in air is very unlikely at room temperature. ${ }^{53}$ However, when working at cryogenic temperatures, sticking efficiency tends to increase and the rate of desorption slows considerably, therefore surface contamination becomes an even more important issue. All UHV best practices are followed both in construction and maintenance of the apparatus. Base pressure in the surface scattering chamber is $6 \times 10^{-11}$ Torr. 
Table 2 Values calculated for air at $293 \mathrm{~K}$ and assume sticking coefficient of 1 and $\xi=3 \times 10^{-10} \mathrm{~m}$. Monolayer formation time shows clearly that vacuum conditions in the ultrahigh vacuum regime are necessary to achieve control over the surface for any experimentally useful period of time.

\begin{tabular}{|r|c|c|c|c|c|}
\hline & $\begin{array}{c}\text { Pressure (Torr) } \\
\text { (Lower limit) }\end{array}$ & $\begin{array}{c}\text { Number } \\
\text { Density } \\
\left(/ \mathrm{cm}^{3}\right)\end{array}$ & $\begin{array}{c}\text { Mean Free } \\
\text { Path }(\mathrm{cm})\end{array}$ & $\begin{array}{c}\text { Surface Collision } \\
\text { Frequency } \\
\left(\mathrm{cm}^{-2} \mathrm{~s}^{-1}\right)\end{array}$ & $\begin{array}{c}\text { Time to } \\
\text { Monolayer } \\
\text { Formation } \\
(\mathrm{sec})\end{array}$ \\
\hline Atmosphere & 760 & $2.7 \times 10^{19}$ & $7 \times 10^{-6}$ & $3 \times 10^{23}$ & $3.3 \times 10^{-9}$ \\
\hline Rough Vacuum & $10^{-3}$ & $3.5 \times 10^{13}$ & 5 & $4 \times 10^{17}$ & $2.5 \times 10^{-3}$ \\
\hline High Vacuum & $10^{-6}$ & $3.5 \times 10^{10}$ & $5 \times 10^{3}$ & $4 \times 10^{14}$ & 2.5 \\
\hline Ultra-High Vacuum & $10^{-12}$ & $3.5 \times 10^{7}$ & $5 \times 10^{6}$ & $4 \times 10^{11}$ & $2.5 \times 10^{3}$ \\
\hline
\end{tabular}

Achieving UHV conditions in the surface scattering chamber while the molecular beam is on is accomplished by extensive use of differential pumping and by baking out the entire vacuum chamber. Differential pumping is necessary in our chamber as the pressure requirements in the surface scattering chamber $\left(\leq 1 \times 10^{-10}\right.$ Torr) are drastically different from the pressure limitations imposed by the molecular beam source $\left(\geq 1 \times 10^{-6}\right.$ Torr). The concept of differential pumping is to separate the region of high pressure from the region of desired low pressure by multiple individually pumped regions between which only small conductance is possible. Our apparatus is separated into four differentially pumped regions. Starting at the molecular beam source these are referred to as the source chamber, hexapole chamber, decelerator chamber, and scattering chamber, as shown in Figure 3. Figure 12 schematically illustrates the vacuum setup used in the apparatus. Differential pumping is used for every vacuum chamber except the TPD apparatus. Differentially pumped rotary feedthroughs (DPRFs) are also used on the surface scattering chamber for reducing pressure spikes associated with rotatable feedthroughs. 
The source, decelerator and scattering chambers are pumped by $685 \mathrm{l} / \mathrm{s}$ turbomolecular pumps (TMP, Pfeiffer HighPace700), while the hexapole chamber is pumped by a 355 1/s TMP (Pfeiffer HighPace 400). The source and hexapole chamber are connected by a skimmer (Beam Dynamics, Inc. Molecular Beam Skimmer Model 2, Nickel) with $2 \mathrm{~mm}$ orifice diameter. The other vacuum regions are connected by $2 \mathrm{~mm}$ diameter circular holes with filleted edges. The outlets of all TMPs, except that pumping the scattering/preparation chamber are connected directly to the fore-vacuum line. The fore-vacuum line is evacuated by three parallel oil-free diaphragm pumps (Pfeiffer MVP 70, 65 1/min) to a pressure of around 0.6 Torr. The best final pressure which can be achieved by a TMP backed by a diaphragm pump is on the order of $10^{-10}$ Torr, due to the limited compression (i.e. ratio of inlet pressure to outlet pressure) of approximately $10^{10}$. For that reason, the outlet of the scattering chamber TMP is connected to the fore-vacuum line via a smaller TMP (Pfeiffer HighPace 80, 38 1/s), giving the larger TMP a backing pressure of $10^{-5}$ mbar. The small TMP is also used to evacuate a gas line used to feed neon to a leak valve for surface sputtering, as well as for pumping out the first stage of the two DPRFs. The second stage of these differentially pumped rotary feedthroughs is connected directly to the decelerator chamber to provide a backing pressure of around $10^{-10}$ Torr. Forevacuum pressure is monitored using three Pirani gauges (Pfeiffer TPR 280) mounted near the outlet of the TMPs. Pressure in the vacuum chambers and upstream from the small backing TMP is measured by bakeable cold cathode gauges that have a lower limit of $2 \times 10^{-9}$ mbar (Pfeiffer IKR 060) for the source, hexapole, decelerator, and preparation chamber, and $1 \times 10^{-11} \mathrm{mbar}$ (Pfeiffer IKR 070) for the scattering chamber. No Bayard-Alpert type gauges have been installed as they rely on hot filaments and could therefore increase the number of charged particles which might cause discharges near high voltage parts. All pressure readings are displayed using two control units (Pfeiffer Maxi Gauge TPG 256A), and logged in 15 minute intervals. A residual gas analyzer (Stanford Research Systems RGA 200) is used in the scattering chamber to monitor the composition of the background pressure in the chamber. The RGA is also used to perform temperature programmed desorption (TPD) experiments as will be described in section 2.2.2.3. 


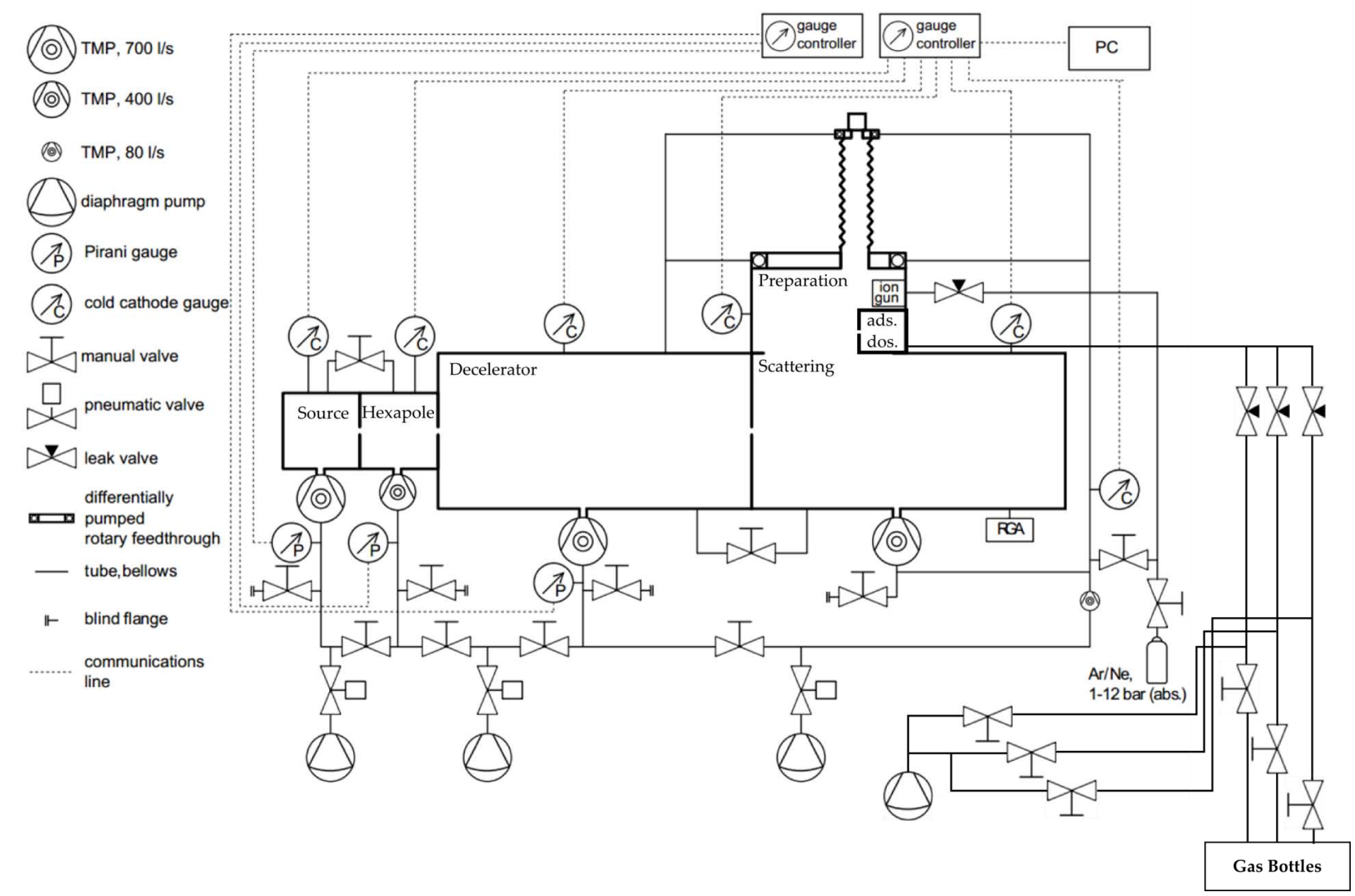

Figure 12 Schematic of vacuum system, adapted from Grätz ${ }^{48}$. Adsorbate doser is abbreviated ads. dos. Details can be found in section 4.2 .3 For more information, see text. 


\subsubsection{Sample mount}

The sample surface is a $1 \mathrm{~cm}$ diameter cylindrical Au crystal cut along the (111) plane (MaTecK). The sample is mounted at the end of a 55 inch oxygen free high conductivity copper (OFHC) coldfinger connected to a closed-cycle Gifford-McMahon helium cooler (ARS Cryo CS-204AB). The expander and coldfinger are mounted on a commercial 4-axis manipulator (VG-Scienta Omniax MXZ800 and MT211B6S) with $800 \mathrm{~mm}$ travel in the vertical axis, $25 \mathrm{~mm}$ travel on the two horizontal axes, and full $360^{\circ}$ rotation around the vertical axis realized with a small differentially pumped rotary feedthrough (DPRF, VG Scienta ZRP100H, DN 100CF), hereafter referred to as the small DPRF, mounted atop the manipulator.

The crystal itself is mounted between two tungsten wires which are connected via stainless steel screws to OFHC copper blocks, see Figure 13. These two OFHC Cu blocks are affixed to a central OFHC $\mathrm{Cu}$ block via $1 \mathrm{~mm}$ thick sapphire disks. The central copper block is then affixed to the end of the coldfinger with a single stainless steel $1 / 4-28$ bolt. A thin $(0.1 \mathrm{~mm})$ foil of silver is place between the sample mount and bottom of the coldfinger before the bolt is tightened. This soft foil deforms under pressure and increases surface contact and therefore thermal conductivity between coldfinger and sample holder. The sapphire spacers serve two purposes. First, the sapphire is used to electronically insulate the surface from the coldfinger. This allows for resistive sample heating by running current through the tungsten filaments. Second, because the thermal conductivity of sapphire is extraordinarily high at low temperatures and decreases with increasing temperature, the surface is very strongly thermally coupled with the coldfinger at low temperatures and only poorly coupled at high temperatures, minimizing the thermal load on the He cooler during sample heating. After heating the surface to $1000 \mathrm{~K}$ for 40 minutes it takes only 20 minutes for the surface to return to $20 \mathrm{~K}$. Crystal temperature is monitored via a chromelconstantan (E-type) thermocouple, the junction of which is placed into a $0.5 \mathrm{~mm}$ diameter hole cut $5 \mathrm{~mm}$ deep in the side of the crystal and staked in with a gold wire. The thermocouple wires run through a double bore $\mathrm{Al}_{2} \mathrm{O}_{3}$ rod mounted within the central $\mathrm{Cu}$ block to extension wires mounted along the coldfinger.

Resistive heating is accomplished using a Genesys 8-180 power supply controlled by LabVIEW software. In order to heat the surface linearly for temperature programmed desorption experiments, the surface heating rate is controlled by referencing a proportional feedback loop to 
the surface temperature. Briefly, heating is begun at a chosen current, $I_{\text {initial}}$, and the thermoelectric voltage from the surface thermocouple is then referenced to an electronic ice point (Omega, MCJ-E) and fed into a 16-bit analog to digital converter (ADC, NI-9215) where it is converted into a digital signal. The temperature corresponding to the thermoelectric voltage is then calculated and recorded as a function of time. The heating rate, $k_{\text {actual }}$, is determined based on successive time/temperature points and compared to the chosen heating rate $k_{\text {chosen,. The }}$ adjusted heating current, $I_{\text {adjusted }}$, is then calculated according to the formula:

$$
I_{\text {adjusted }}=\frac{k_{\text {actual }}}{k_{\text {chosen }}} \times P
$$

where $P$ is a constant of proportionality determined empirically, and the new current is sent to the power supply. Typical values for $P$ are between 0.001 and 0.003 . Larger values of $P$ are necessary for lower temperature ranges. Using this proportional feedback program, temperature ramps between $k=0.08 \mathrm{~K} / \mathrm{s}$ and $k=1.67 \mathrm{~K} / \mathrm{s}$ are possible. See the appendix for a schematic of the labVIEW program used to control the surface temperature. 


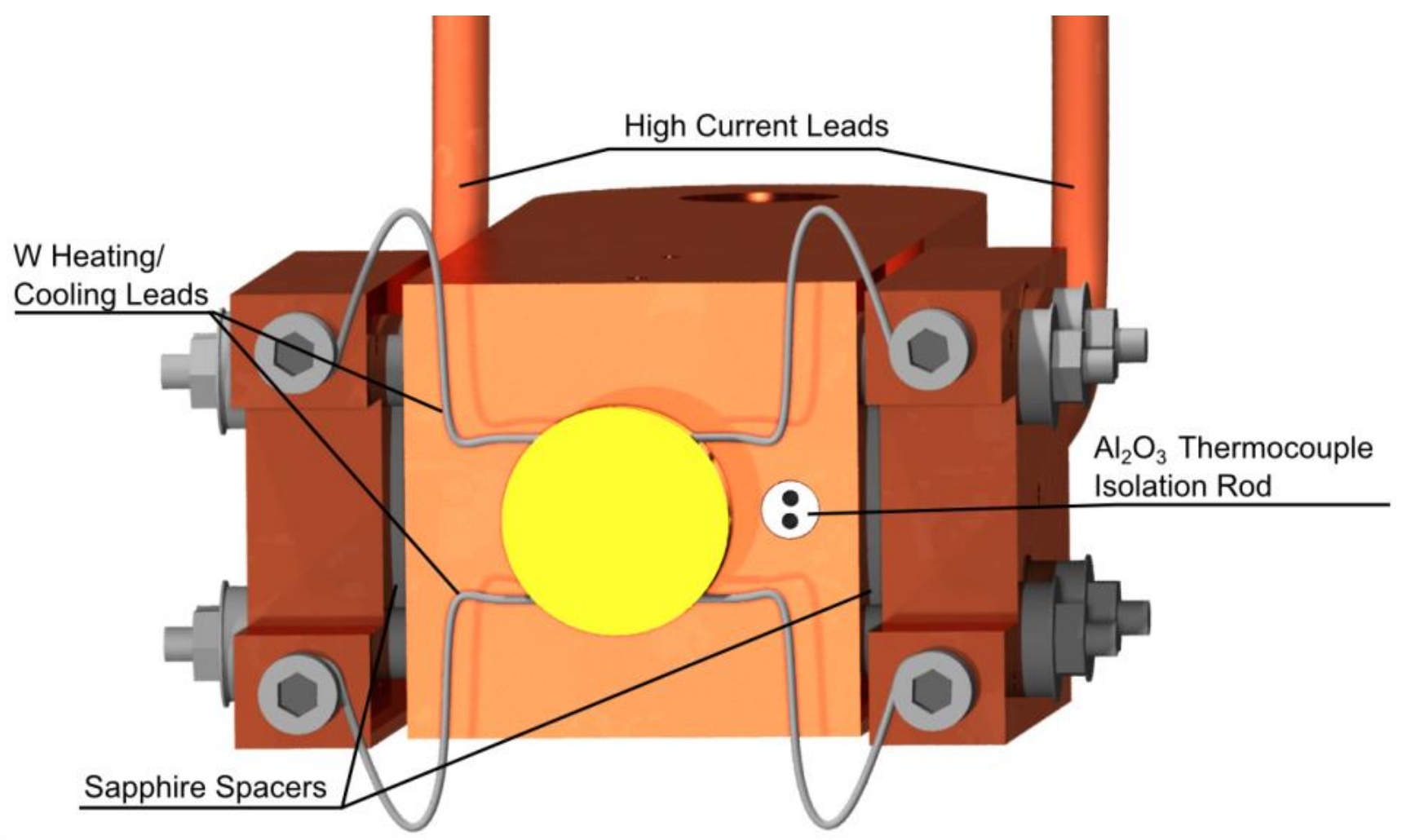

Figure 13 CAD drawing showing details of front face of the sample holder. The Au(111) surface is isolated electronically from the main body by sapphire spacers. The sample itself is suspended between preformed tungsten wires, which also serve as heating and cooling leads. Surface temperatures can be controlled between 19-1337 K.

Surface temperature has been calibrated after the procedure outlined by Schlichting and Menzel. ${ }^{54}$ In order to calibrate the temperature of their $\mathrm{Ru}(0001)$ sample, they compared the desorption rates of thick overlayers (>5 ML) of several gases to the equilibrium pressure of the gas condensates. This is reasonable because the sticking coefficients of gases on their own solids is unity, the desorption of multilayers is zeroth order, and the desorption energy is independent of layer thickness above $5 \mathrm{ML}$. It was found that the uncalibrated temperature scale could be brought into coincidence with the calibrated scale by a voltage offset. In order to calibrate the temperature of our surface, we recorded TPD spectra for thick overlayers of several gases and compared them to published spectra of similar doses at the same heating rate. It was found that by applying a voltage offset, we could reproduce the peak desorption temperatures for the thick overlayers of $\mathrm{Ar}, \mathrm{Kr}, \mathrm{Xe}, \mathrm{SF}_{6}, \mathrm{NO}, \mathrm{N}_{2}$, and $\mathrm{CO}$ within $\pm 1.5 \mathrm{~K}^{54,55}$ 
The surface is cleaned by standard sputter-anneal cycles until no contamination is detectable by Auger electron spectroscopy. We clean the surface daily by sputtering $(\approx 25$ $\mu \mathrm{A} / \mathrm{cm}^{2}$ ) with $3 \mathrm{kV} \mathrm{Ne}$ atoms for 15-20 minutes and subsequent annealing at $1000 \mathrm{~K}$ for 20 minutes. Our decision to sputter with $\mathrm{Ne}^{+}$rather than $\mathrm{Ar}^{+}$ions is due to the low temperature coldfinger. After 20 minutes sputtering with $\mathrm{Ar}^{+}$ions at $2 \times 10^{-7}$ mbar background pressure, pressure returns to base pressure only after 40-60 minutes due to adsorbed Ar slowly desorbing from the $20 \mathrm{~K}$ coldfinger creating a short term virtual leak. Ne on the other hand, desorbs quickly from the cold copper and the chamber returns to base pressure within 5 minutes after sputtering with neon.

The manipulator is mounted vertically atop a CF 13.25 inch DPRF (Thermionics RNN1000/MS), hereafter referred to as the big DPRF. The manipulator is mounted off-center from the main vertical axis of rotation in order to allow for a variety of different experimental geometries. As shown in Figure 14, the sample holder is offset from the coldfinger so that when the surface is oriented toward the center of the chamber, the front face of the crystal lies on the central rotation axis of the big DPRF. In this way, we can vary scattering angle relative to the molecular beam from $0-90^{\circ}$ by rotating the big DPRF. When characterizing and preparing the surface, we rotate the small DPRF such that the surface points directly away from the center of the chamber and rotate the big DPRF to position the surface in front of the different instruments mounted around the perimeter of the upper tier. Shown in Figure 14 is a schematic of the sample holder rotational movement in the scattering and preparation chambers. 

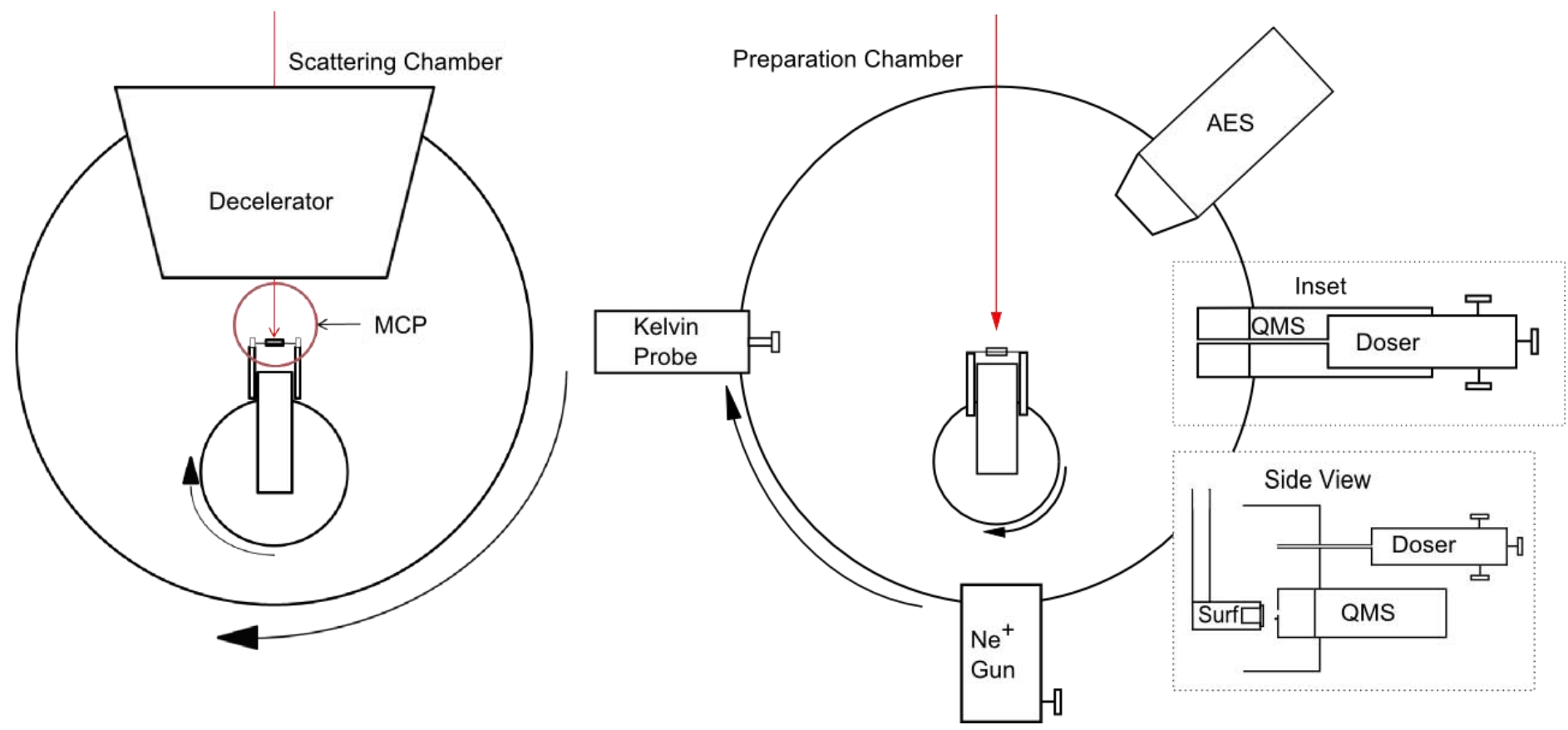

Figure 14 The left panel shows position of sample surface in front of molecular beam in the lower tier of the surface chamber. Rotation of big and small differentially pumped rotary feedthroughs is indicated. MCP: microchannel plate detector. The right panel shows the upper tier of the scattering chamber, the preparation chamber. QMS: quadropole mass spectrometer, Doser: leak valve manifold with gas cell for targeted adsorbate dosing, AES: Auger electron spectrometer. 


\subsubsection{Adsorbate decorated surface}

Initial experiments performed with this machine investigated the absolute emission probability of electrons emitted when $\mathrm{CO}^{*}$ quenches at a clean gold surface as detailed in section 3.2. In order to further characterize the processes occurring at the surfaces, we manipulated the surface interaction region directly by creating an adsorbate covered $\mathrm{Au}(111)$ surface. In order to characterize the temperature dependence of adsorbate coverage, we designed and constructed a temperature programmed desorption (TPD) apparatus based on a quadrupole mass analyzer (Stanford RGA-200) and the temperature control offered by our sample mount. The following section will first present an introduction into the nature of atomic and molecular adsorption at a metal surface and then present a theoretical and experimental explanation of a TPD experiment and introduce the experimental apparatus with which these experiments were carried out.

\subsubsection{Adsorption}

Adsorption is a process by which a gas phase atom or molecule loses some of its translational energy and becomes bound to a surface. Adsorption processes are in integral step in nearly all surface related technological processes. The most direct example is that of heterogeneous catalysis. In most heterogeneous catalytic processes, reactants need to adsorb to the surface before reaction can occur. There are two main classes of surface catalyzed reaction mechanisms, Langmuir-Hinshelwood and Eley-Rideal. Langmuir-Hinshelwood type reactions occur by two reactants first adsorbing on neighboring sites on a surface, after which the adsorbates react with one another (still on the surface), and desorb from the surface after forming a new molecule. For reactions which proceed via an Eley-Rideal type mechanism, only one reactant adsorbs on the surface and the second react with the adsorbed reactant directly from the gas phase, leading to desorption. Both reaction mechanisms crucially depend on one or both reactants first adsorbing to the surface of the catalyst.

Energy loss can proceed through a variety of loss channels as shown in Figure 1. Depending on the amount of energy lost to the surface, impact of an atom or molecule can excite phonons on the surface, induce emission of a photon, and/or excite electron-hole pairs. If the particle loses more energy than the surface's work function, the impact can induce emission of an electron into vacuum. If enough translation energy is lost by the incident particle, it can 
become trapped in the attractive well near the surface. The discussion of adsorption in this chapter follows the treatment developed by Zangwill ${ }^{56}$ and Gross. ${ }^{56,57}$

The potential energy surface (PES) is of central importance for any theoretical description of adsorption. A PES describes the energy hyperplane over the configuration space of the atomic coordinates of the involved atoms. In Figure 15, the horizontal axis represents the distance, $z$, between surface $M$ and the diatomic molecule $A B$. The vertical axis represents the potential energy of the system. Two curves are plotted: the green curve is labeled $A B+M$ and at large molecule-surface distances represent the potential energy of a diatomic molecule at a macroscopic distance from a metal surface (M). The shallow minimum in the green curve occurs at longer molecule-surface distance and represents the physisorption energetic well, $\mathrm{AB}_{\text {phys }}$.

The blue curve, $\mathrm{A}+\mathrm{B}+\mathrm{M}$, represents the interaction of two widely separated atoms with the surface. At the potential minimum labeled $A+B_{\text {chem }}$, the curve represents the energy of atoms $\mathrm{A}$ and $\mathrm{B}$ bound individually to the surface after the molecular chemical bond has been broken. This is known as dissociative chemisorption. Formation of chemical bonds with the surface often requires a rearrangement of the molecule's nuclear coordinates. Therefore, if the nuclei were frozen in the chemisorbed geometry, then the potential energy curve would rise above the zero energy as in the blue curve. At large values of $z, A+B+M$ represents the energy of atoms $\mathrm{A}$ and $\mathrm{B}$ equidistant from the surface and also well spaced from one another. Therefore, the energetic difference between $\mathrm{AB}+\mathrm{M}$ and $\mathrm{A}+\mathrm{B}+\mathrm{M}$ represents the dissociation energy of the molecule in free space, $D(\mathrm{~A}-\mathrm{B})$. Pure physisorption is understood to involve bond energies from $0-0.25 \mathrm{eV}$ while chemisorption tends to have bond energies $>1 \mathrm{eV}$.

The position of the crossing of the two potentials determines whether chemisorption is activated. In Figure 15, there is a significant activation energy to chemisorption, denoted $E_{\mathrm{a}}^{\text {ads }}$. The activation energy of desorption of the recombined chemisorbed molecule is denoted $E_{\mathrm{a}}^{\text {des }}$. The desorption activation energy of the physisorbed molecule (or atom) is, to a very good approximation, equal to the adsorption energy. This relationship will be explored in more detail in section 3.1. It should be pointed out that while it is a useful model for developing an instinct about adsorption, this one-dimensional model neglects the angular orientation of the incoming molecule, changes in the internal bond length of the molecule, and the position of the molecule parallel to the surface plane, i.e. proximity to different binding sites on the surface. 


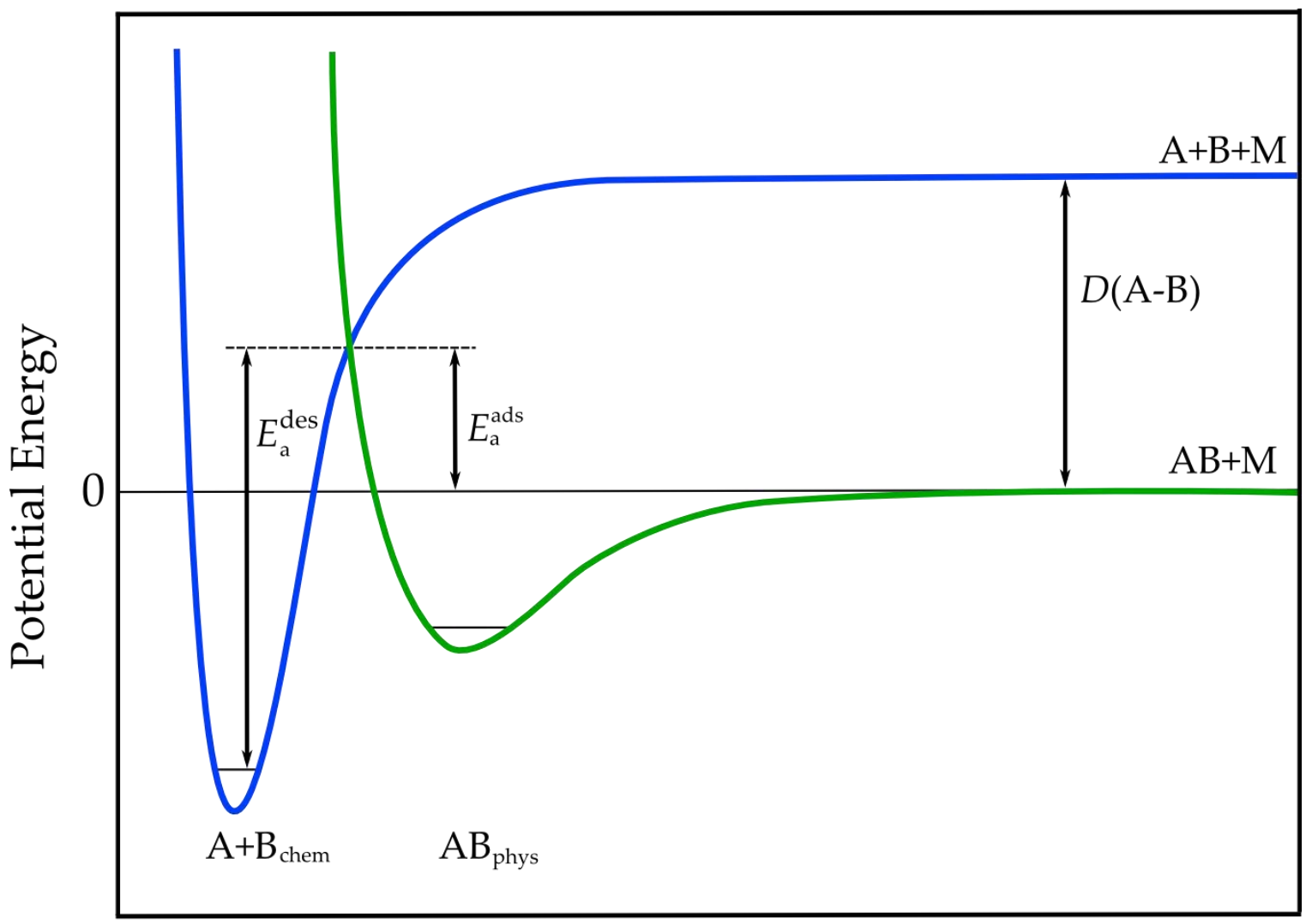

$z$

Figure 15 Two-dimensional potential energy diagram of molecule-surface interaction as a function of molecule-surface distance. The green curve represents physisorption and the blue curve dissociative chemisorption. There is a substantial barrier to dissociative chemisorption. $\mathrm{A}+\mathrm{B}+\mathrm{M}$ at large values of $z$ represents the energy of two atoms of a diatomic molecule a long distance from the surface and at long distance from each other. Therefore the energy difference between the green and blue curves at high $\mathrm{z}$ values is equal to the dissociation energy of a diatomic molecule. For the case of atomic adsorption, only the green curve is relevant. See text for more discussion.

The shallow adsorption well in the green curves of Figure 15 is known as physisorption and is a result of van der Waal's attractions. For a polarizable atom close to a surface, a mutual attraction between the atom and the surface exists that arises from the interaction of the polarizable solid with dipolar quantum mechanical fluctuations of the atomic charge distribution. In other words, atomic electrons are attracted to their image in the solid. ${ }^{56}$ The steep rise in the physisorption curve as the molecule approaches the surface is a result of Pauli repulsion between wave functions of the molecule and substrate. Pure physisorption is characterized by binding 
energies of less than $\approx 0.25 \mathrm{eV}$ and very little perturbation to the electronic structure of the adsorbate. The elementary step in physisorption from the gas phase does not involve an activation energy, as is clear from Figure 15. Under appropriate conditions, gas phase molecules can also physisorb in multilayers. That is to say, an arbitrary number of adsorbate layers can physisorb atop the layer bound directly to the substrate. This behavior will be characterized for a number of different adsorbate gases in section 3.1. Because the physisorption bond is relatively weak, adsorbate-adsorbate interactions can dominate adsorbate-substrate forces. This can lead to, among other things, formation of incommensurate monolayer geometries in which the overlayer and substrate have independent lattices. For physisorbed adsorbate layers, the densest possible packing is determined by the adsorbate's van der Waals radius. ${ }^{58}$

Because of the chemically unreactive nature of noble gases, the adsorption of noble gases on metal surfaces has long been considered prototypical physisorption system. ${ }^{59-63}$ Pure physisorption arises solely due to the induced dipole moment of a nonpolar adsorbate interacting with its own image charges in a polarizable solid, i.e. van der Waals forces. See Figure 16 for the example of a hydrogen atom close to the surface of a perfect conductor. ${ }^{57}$ It must be noted that while the hydrogen atom provides a useful model system from a theoretical point of view, practically speaking it can be highly reactive at surfaces and pure non-reactive physisorption cannot be expected. 

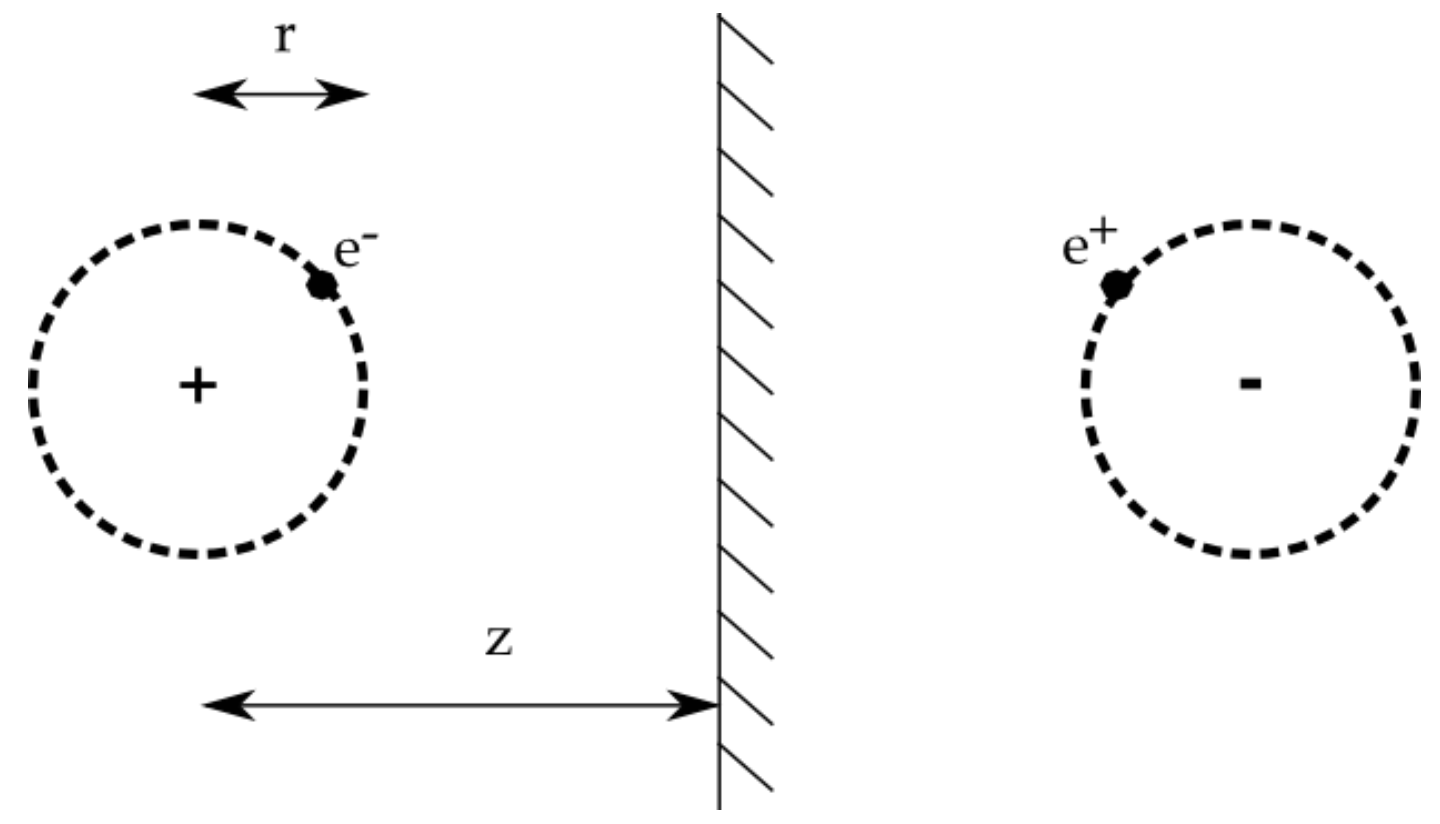

Figure 16 Schematic representation of a hydrogen atom in front of a perfect conductor. The physisorption bond between atom and metal surface arises due to the atom's interaction with its own image charges. Adapted from reference ${ }^{57}$.

The total electrostatic energy of the hydrogen/perfect conductor system is the sum of two attractive and two repulsive terms:

$$
W=\frac{1}{2}\left[-\frac{e^{2}}{2 z}-\frac{e^{2}}{2(z-r)}+\frac{e^{2}}{2 z-r}+\frac{e^{2}}{2 z-r}\right]
$$

Where $z$ is the distance from atom to surface, $r$ is the projection of the electron's orbital motion along the normal to the surface, and $e$ is the elementary charge. The factor of one-half describes the electric field vanishing within the conductor. ${ }^{56}$ The first term of a Taylor expansion of equation 2.13 in powers of $r / z$ yields the following approximation for $W$, the total electrostatic energy:

$$
W=-\frac{1}{8} \frac{e_{0}^{2} r^{2}}{z^{3}}
$$

where $r$ is the radius of the electron orbit of the $\mathrm{H}$ atom and $\mathrm{z}$ the distance from atomic to the conductor surface. The numerator is the product of the dipole moments of the atom and its 
image. The fact that the electrostatic energy is proportional to $r^{2}$ accounts for the fact that large atoms can be more strongly polarized and therefore physisorb more strongly. $W$ is inversely proportional to $z^{3}$. This $z$ dependence differs from the attractive term in the familiar gas phase van der Waals expression, which is proportional to $z^{-6}$. The gas phase $z^{-6}$ dependence arises from the Boltzmann-statistically weighted averaging of the interaction over all orientations of two rotating dipoles. The $z^{-3}$ dependence of attraction of a polarized atom with its image is reasonable considering that the oscillating dipole of the $\mathrm{H}$-atom and its image are always perfectly in phase.

In contrast to physisorption, the chemisorption bond is characterized by the creation of chemical bonds between adsorbate and substrate. Bond energies for chemisorption are often greater than $1 \mathrm{eV}$. Different chemisorption bonds can be further classified as covalent, ionic, or metallic. ${ }^{57}$ While physisorption occurs in any solid/gas or solid/liquid system, chemisorption is chemically specific. As chemisorption involves significant changes in the adsorbate's electronic state, they can be detected by appropriate physical means. Chemisorption, just as in other chemical reactions, often involves an activation energy.

The reader should note that there is an undefined area for bond energies between 0.25 and $1 \mathrm{eV}$. The strength with which a molecule bonds to surface can be a factor of both van der Waals forces and some charge transfer between surface and molecule. In these cases, it is difficult to characterize the particle-surface bond as either pure chemisorption or pure physisorption.

\subsubsection{Temperature programmed desorption}

Temperature programmed desorption (TPD), also known as thermal desorption spectroscopy (TDS), is a convenient way to determine the binding energy of particles on surfaces. In order to perform a TPD experiment, a low temperature surface is prepared by exposing it to a flux of adsorbate particles until the desired adsorbate coverage is reached. The TPD experiment consists of monitoring the partial pressure of the adsorbate versus time during a linear temperature ramp of the surface. This partial pressure measured at one mass/charge is proportional to instantaneous partial pressure of the desorbing species in the chamber. The partial pressure is proportional to the rate of desorption of the adsorbate from the surface, which is equal to the rate at which surface coverage is depleted. 
The rate of desorption rises quickly as surface temperature, $T_{\mathrm{S}}$, increases. Therefore, the partial pressure signal of the adsorbate species increases with $T_{\mathrm{S}}$ until the population of adsorbed particles has been exhausted. The partial pressure then drops rapidly, resulting in a sharp peak in a plot of desorption rate versus $T_{\mathrm{S}}$. Particles in different binding states will exhibit different characteristics in the TPD spectrum. For the physisorbed species reported in this work, the particles bound directly to the surface (monolayer) are bound more strongly than those bound in the second layer above the surface (bilayer). This difference in desorption characteristics results in a distinct peak for each adsorbate binding state. Individual desorption peaks can also arise from particles bound in the same adsorbate layer, but at different binding geometry, i.e. two- or three-fold binding sites versus on-top binding. Due to the flat, uncorrugated, homogeneous nature of $\mathrm{Au}(111)$, this phenomenon does not arise in the results discussed in this work.

Thermal desorption is often described with an expression known as the Polanyi-Wigner equation, which looks similar to the Arrhenius equation:

$$
r(\Theta)=-\frac{\mathrm{d} \Theta}{\mathrm{d} t}=v \Theta^{n} \exp \left[-E / R T_{\mathrm{S}}\right]
$$

where $r$ is the rate of desorption, $\Theta$ the adsorbate coverage, $t$ the time, $v$ the pre-exponential factor of desorption, $n$ the order of desorption, $R$ the gas constant and $T_{\mathrm{S}}$ the surface temperature. $\mathrm{d} T_{\mathrm{S}} / \mathrm{d} t=k$, where $k$ is the heating rate. ${ }^{64}$

The order of desorption, or kinetic order, is a sign of the coverage dependence of the desorption rate. In zeroth-order systems the coverage term, $\Theta^{n}$, becomes unity and the desorption rate is independent of coverage. In this case, desorption rate increases exponentially with $T_{\mathrm{S}}$ until the pressure drops very rapidly after all molecules have desorbed, as shown in the Figure 17. Hallmarks of zeroth order desorption are a common leading edge for all spectra independent of initial coverage, a rapid pressure drop after all molecules have been desorbed, and higher peak desorption temperature $\left(T_{\max }\right)$ for higher initial coverage. Zeroth order kinetics are observed in thick multilayers, where supply of desorbing particles is essentially infinite.

As will be shown in section 3.1 rare gas adsorbed on $\mathrm{Au}(111)$ exhibits quasi-zeroth order behavior. This is an indication that the adsorbate-adsorbate interactions are attractive. This 
attractive force between adsorbates leads to island formation and desorption occurs preferentially at island edges, leading to identical desorption conditions regardless of coverage.

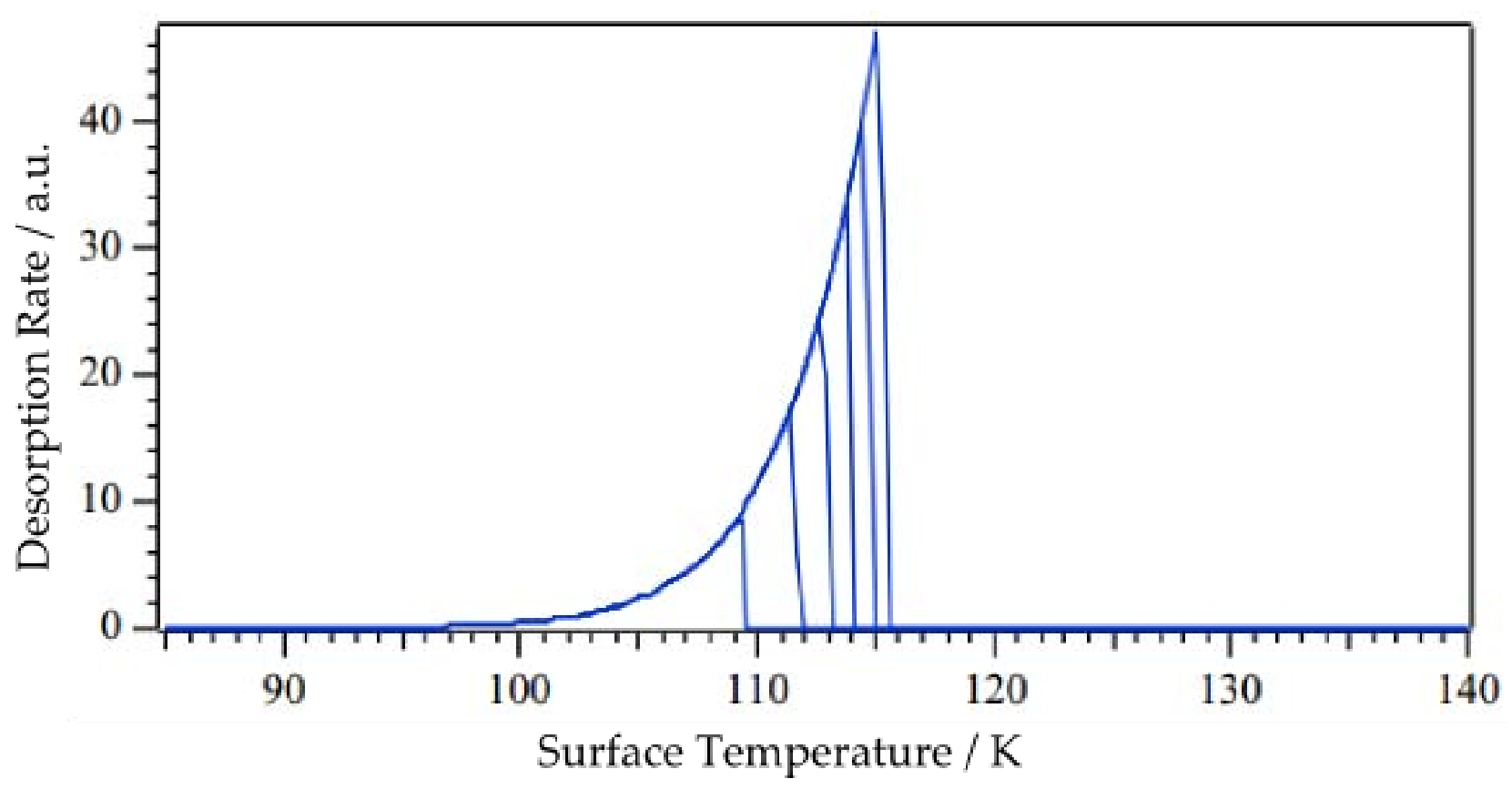

Figure 17 Simulated zeroth order TPD spectra for different initial adsorbate coverages. Note the common leading edge for all spectra, indicating that desorption rate is independent of coverage. Peak desorption temperature increases with increasing coverage. ${ }^{65}$

First order desorption kinetics imply that the desorption rate is proportional to instantaneous coverage. TPD spectra exhibiting first-order kinetics show a constant $T_{\max }$ independent of initial coverage and a characteristic asymmetric peak shape indicating a balance between $\theta$ and $\exp \left[-E / R T_{S}\right]$ terms in the Polanyi-Wigner relation. Simulated first order spectra are presented in Figure 18. Atomic or non-recombinative molecular desorption is often of first order. 


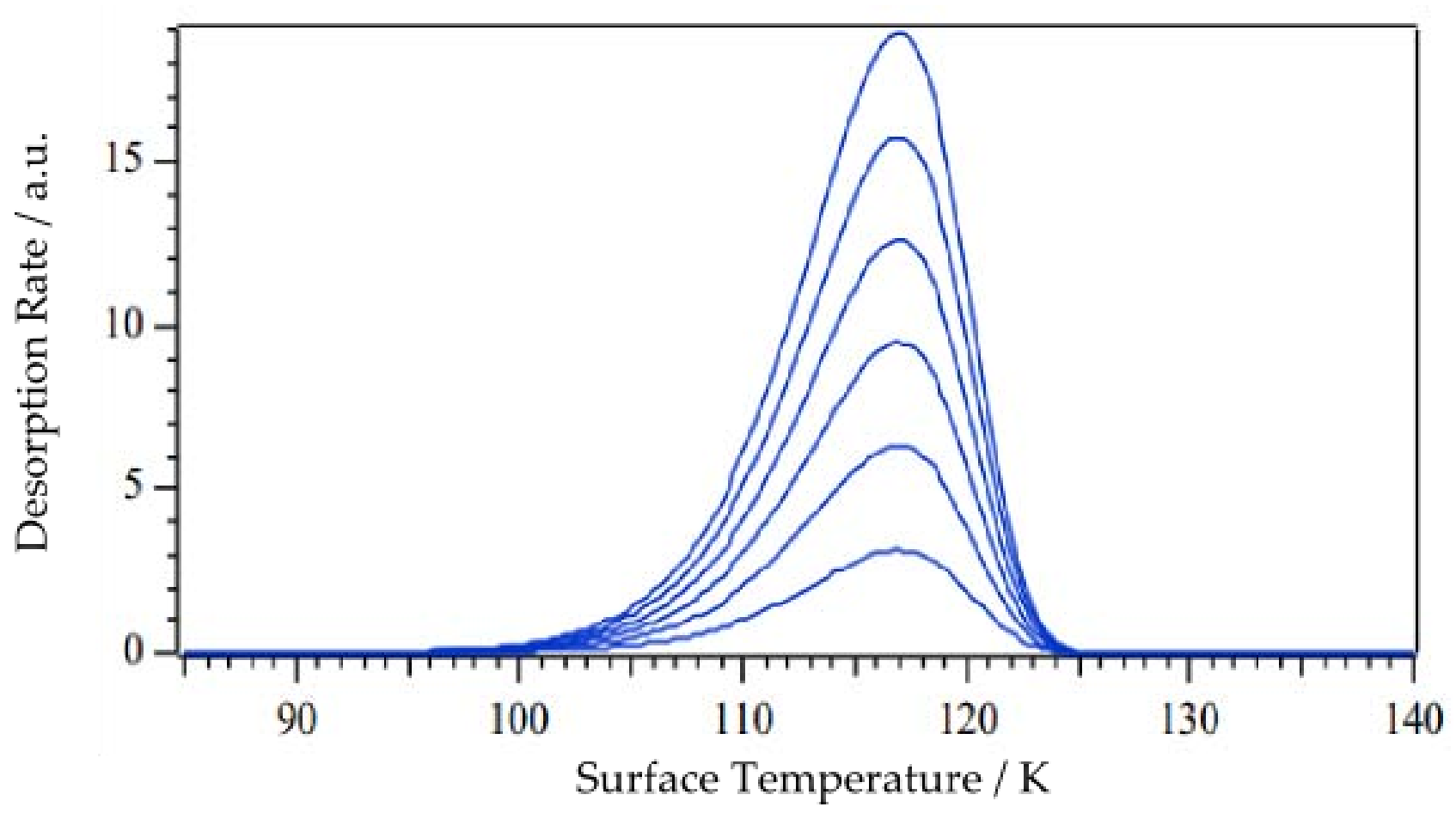

Figure 18 Simulated first order TPD spectra for different initial adsorbate coverages. Note the common $T_{\max }$ for all spectra and characteristic asymmetric peak shape. ${ }^{65}$

Second order desorption kinetics indicate that desorption rate is proportional to the square of instantaneous adsorbate coverage. Like first order kinetics, second order desorption peaks also shows a certain balance between the $\theta$ and $\exp \left[-E / R T_{\mathrm{S}}\right]$ terms of (2.15), but the peak shape is more symmetric with $T_{\max }$ shifting to higher temperatures at lower coverages, as shown in Figure 19. Second order desorption kinetics are sometimes observed for processes of recombinative desorption, i.e. hydrogen desorbing from $\operatorname{Ru}(001){ }^{66}$ 


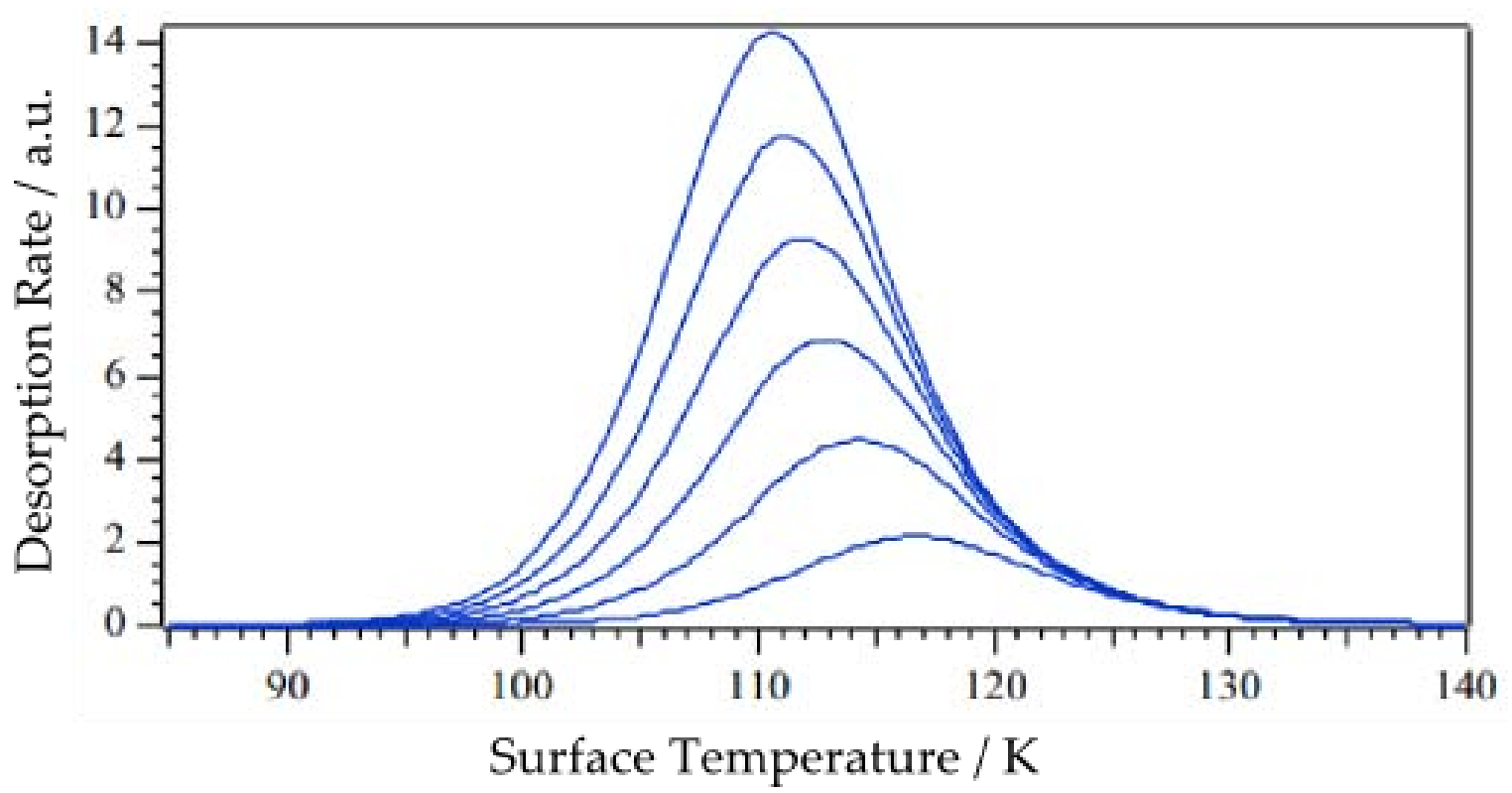

Figure 19 Simulated second order TPD spectra for different initial adsorbate coverages. Note $T_{\max }$ decreases with increasing coverage and lack of common leading edge. ${ }^{65}$

TPD spectra can be analyzed in a number of different ways in order to determine the dependence of desorption rate on coverage, $\theta$, desorption order, $n$, the desorption activation energy, $E_{\mathrm{des}}$, and the Arrhenius prefactor, $v$. For adsorbates that desorb completely below surface temperatures of $200 \mathrm{~K}$ and whose bond with the surface is dominated by dispersion forces, $E_{\mathrm{des}}$ is an excellent proxy for the adsorbate binding energy to the surface, as will be shown in section 3.1. The desorption activation energy of chemisorbed molecules, however, is dependent on the position of the curve crossing between the physisorbed and chemisorbed states. When charge transfer plays a role in the adsorbate-surface bond, $E_{\text {des }}$ cannot be directly compared to the depth of the binding well. ${ }^{67-69}$

The Arrhenius pre-exponential factor, $v$, is normally thought of as the "attempt frequency" of a reaction. For simple chemical reactions, the pre-exponential factor is simply a measure of number of collisions of reactants (whether leading to a reaction or not). For desorption processes, the interpretation of the pre-exponential factor is more nuanced. Within the framework of transition state theory (TST) desorption is thought to proceed via an activated complex possessing two degrees of translational freedom in the plane of the surface. In cases of atomic or monomolecular desorption, the pre-exponential factor, $v$, is described with 


$$
v=\left(T_{\max } / h\right) F_{\mathrm{A}^{*}} / F_{\mathrm{A}}
$$

where $F_{\mathrm{A}^{*}}$ is the partition function of the activated complex, $F_{\mathrm{A}}$ the partition function of the bound state, $h$ is Planck's constant, and $T_{\max }$ is the surface temperature corresponding to the peak in the TPD spectrum. ${ }^{70}$ If the ratio of the total partition functions of the activated complexes and adsorbed particles is assumed to be 1 , then a "normal" pre-exponential factor is on the order of $10^{13} \mathrm{~s}^{-1} .^{71}$ Measured pre-exponential factors for desorption can vary by several orders of magnitude from this normal value, however. It can be shown that a pre-exponential factor on the order of $10^{15} \mathrm{~s}^{-1}$ is indicative of a tightly bound, immobile adsorption state and a pre-exponential factor of $10^{12} \mathrm{~s}^{-1}$ suggests a mobile adsorbate layer. For further discussion, see Yates and Zhdanov. $^{60,70}$

The kinetic parameters $E_{\text {des }}$ and $v$ can be derived from TPD experiments in a number of ways. Numerous reviews have been published and the discussion here will focus only on those methods used in this work. ${ }^{72,73}$ All methods take as a starting point the Polanyi-Wigner equation (2.15). By plotting $\ln \left(r / \theta^{n}\right)$ vs. $1 / T$ for the rising edge of the TPD peak with different values of $n$, one obtains plots from which the desorption activation energy can be derived from the slope and the pre-exponential factor from the $1 / T$-intercept. When comparing the plots for different values of $n$, that value resulting in the best fit to a straight line is the correct order for the desorption process. ${ }^{74} \mathrm{~A}$ second indicator of the correct desorption order is to compare several plots of the same system, assuming the same desorption order, but using different initial coverages. The resulting lines will diverge for those plots assuming incorrect desorption order. This analysis method calculates averages of $E_{\mathrm{des}}$ and $v$ over the entire range of temperatures used. This interpretation is commonly referred to as leading edge analysis as it is commonly applied to only the initial rising edge of the TPD peak in order to assume constant coverage.

A second method of deriving these parameters is possible by beginning with the same initial coverage and performing several TPDs at different heating rates $(k)$. This analysis is only valid for first order desorption processes, but has the advantage that the peak under scrutiny must not be well separated from nearby peaks as the leading edge is not used for calculations, rather the point of maximum desorption rate $\left(T_{\max }\right)$. Using his method, one can plot $2 \ln T_{\max }-\ln k$ vs. $1 / T_{\max }$ and obtain a straight line whose slope is $E_{\mathrm{des}} / R$ and with intercept of $\ln E_{\mathrm{des}} / R v$, where 
$R$ is the gas constant. This method implicitly assumes that the fractional coverage remaining on the surface at $T_{\max }$ is the same for all heating rates.

\subsubsection{TPD apparatus}

TPD experiments are performed using the sample mount described earlier and a modified residual gas analyzer. The residual gas analyzer (Stanford Research Systems RGA-200) is comprised of an electron impact ionization source, quadrupole mass spectrometer, Faraday cup, and channel electron multiplier. Instead of operating with the analyzer with the ionizer directly in the vacuum chamber, the apparatus is mounted within a copper shroud enclosing the ionizer after the design of Feulner and Menzel. ${ }^{75}$ As seen in the CAD drawing of Figure 20, a $1 \mathrm{~mm}$ diameter thin aperture is centered on the front face of the cylindrical cap. During TPD measurements, the front face of the crystal is positioned $1.5 \mathrm{~mm}$ from the front aperture of the cap. Positioning the crystal face close to the aperture of the ionizer shroud geometrically limits gas entering the ionization region to those particles desorbed from the front face of the crystal. There are four larger holes around the perimeter of the cap behind the ionizer through which the RGA is pumped into the surface chamber. There is no differential pumping of this device, so pumping speed is determined exclusively by conductance through the shroud's pumping holes and the volume of the quadrupole chamber.

This setup has two major advantages over an unshielded ionizer for TPD. First, the gas particles entering the ionization region come predominantly from the front face of the crystal, suppressing any spurious peaks that may arise from desorption from other parts of the sample holder such as crystal edges, heating leads, thermocouple, etc. Second, the mass spectrometer's sensitivity is enhanced because pumping speed from the ionization volume is limited by the four $4 \mathrm{~mm}$ pumping holes. This allows the desorbed molecules to make several passes through the ionizer, which increases signal to noise significantly. ${ }^{75}$ 


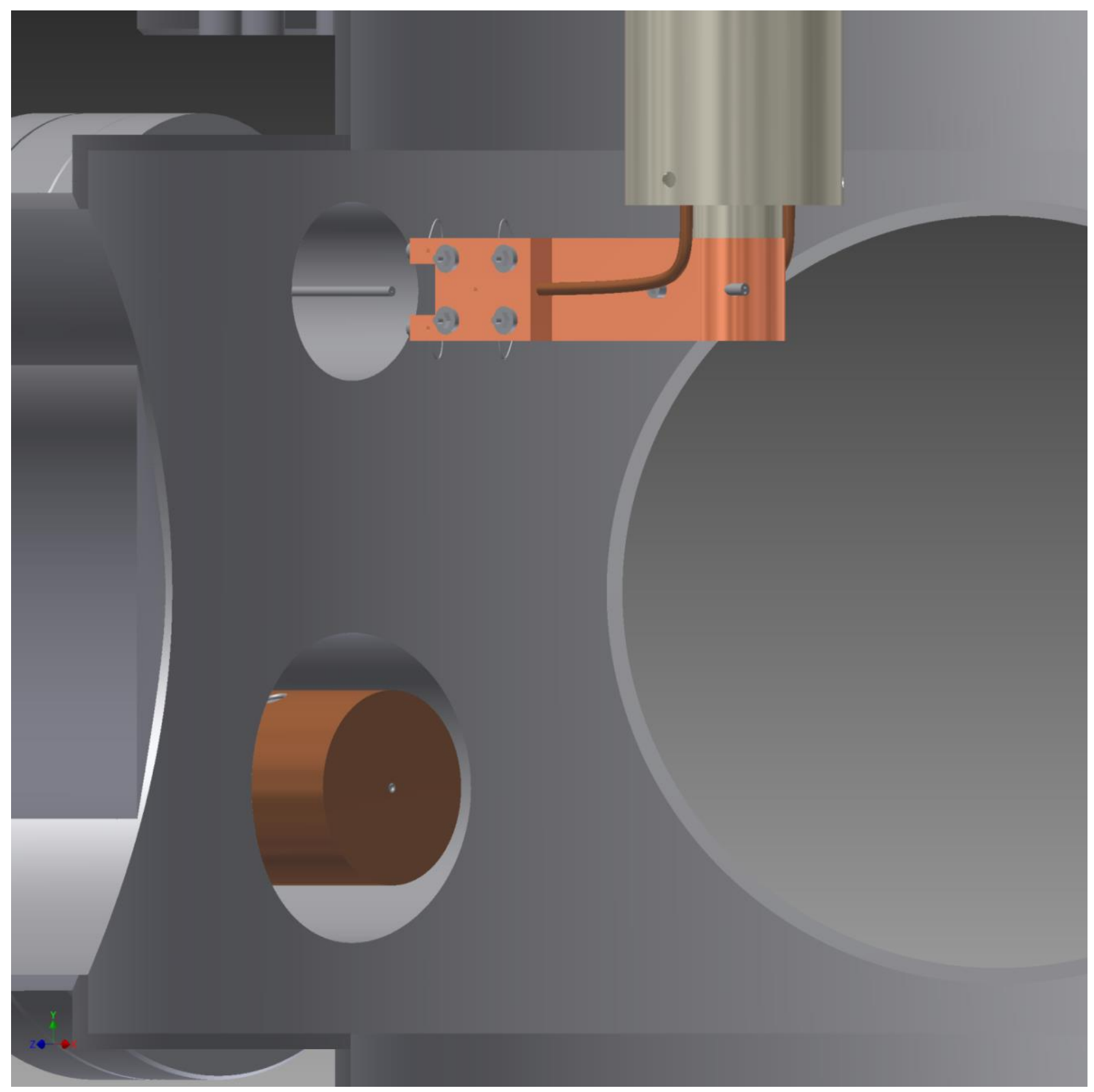

Figure $20 \mathrm{CAD}$ drawing of surface positioned in front of adsorbate doser. The $83 \mathrm{~mm}$ long dosing tube has an inner diameter of $500 \mu \mathrm{m}$. By pressurizing the gas cell behind the doser, adsorbates can be introduced into the chamber with a well-defined, narrow angular distribution. In the lower part of the chamber, the front cap of the residual gas analyzer (RGA) is visible. For TPD measurement, the surface is translated downward until the front face of the crystal is 1.5 $\mathrm{mm}$ from the small aperture in the RGA cap. The $1 \mathrm{~mm}$ aperture on the front face of the cylindrical cap limits gas entering the ionization to that originating from the front face of the crystal. The limited pumping speed enhances signal-to-noise by allowing particles to make multiple passes through the ionizer. 
Adsorbates are introduced onto the surface using a home built surface doser, seen in Figure 20 and schematically in Figure 14. Three UHV precision leak valves (MDC Precision Leak, DN40CF/DN16CF) are used to pressurize a gas cell which is connected to the surface scattering chamber via an $83 \mathrm{~mm}$ stainless steel tube with a $0.5 \mathrm{~mm}$ inner diameter. Pressurizing the gas cell behind the tube causes the gas of interest to leak into the chamber with a narrow, well-defined angular distribution. ${ }^{45}$ By positioning the surface $18 \mathrm{~mm}$ from the aperture, $90 \%$ of the flux leaving the tube will first impinge upon the front face of the crystal before scattering into the chamber. This targeted adsorbate dosing allows us to further suppress any spurious peaks in the TPD spectrum which occur due to desorption from other parts of the sample holder during heating. By monitoring the pressure in the gas cell and ambient pressure in the surface preparation chamber during dosing, empirical "recipes" can be determined for reproducible dosing with accuracy of \pm 0.1 monolayer (ML). 


\subsubsection{Work Function}

The work function is defined as the minimum energy needed to move an electron from within the bulk of a solid to a point in vacuum an infinite distance away from the surface. For a metal, therefore, the work function can be defined as the energy difference between the Fermi level and vacuum. The work function is not a bulk property, rather, it is specific to each surface of a given material. ${ }^{76}$

\subsubsection{Work Function and effect of adsorbates}

When describing the surface work function change induced by an adsorbed atom or molecule, it is useful to first discuss the nature of the work function, $\Phi$. The energy difference between the Fermi level and a singly ionized metal surface and an electron at rest in vacuum can be divided into two contributions, as shown in Figure 21. The first is due to short-range Coulomb interaction - exchange and correlation - which is a pure bulk effect. That is to say, the energy of an electron in the bulk is lowered by the chemical potential, $\bar{\mu}$, due to the chemical bonding which forms the solid. ${ }^{77}$ The second contribution is due to the existence of the surface dipole layer. At the metal-vacuum interface, electron density drops to zero in less than one atomic distance perpendicular to the surface. This rapid drop in electron density is accompanied by some leakage of electron density into vacuum. This spill-out creates an area of negative charge in front of the surface and a concomitant excess of positive charge in the first atomic layer. In this way, a surface dipole is formed with its negative end pointing into vacuum. 

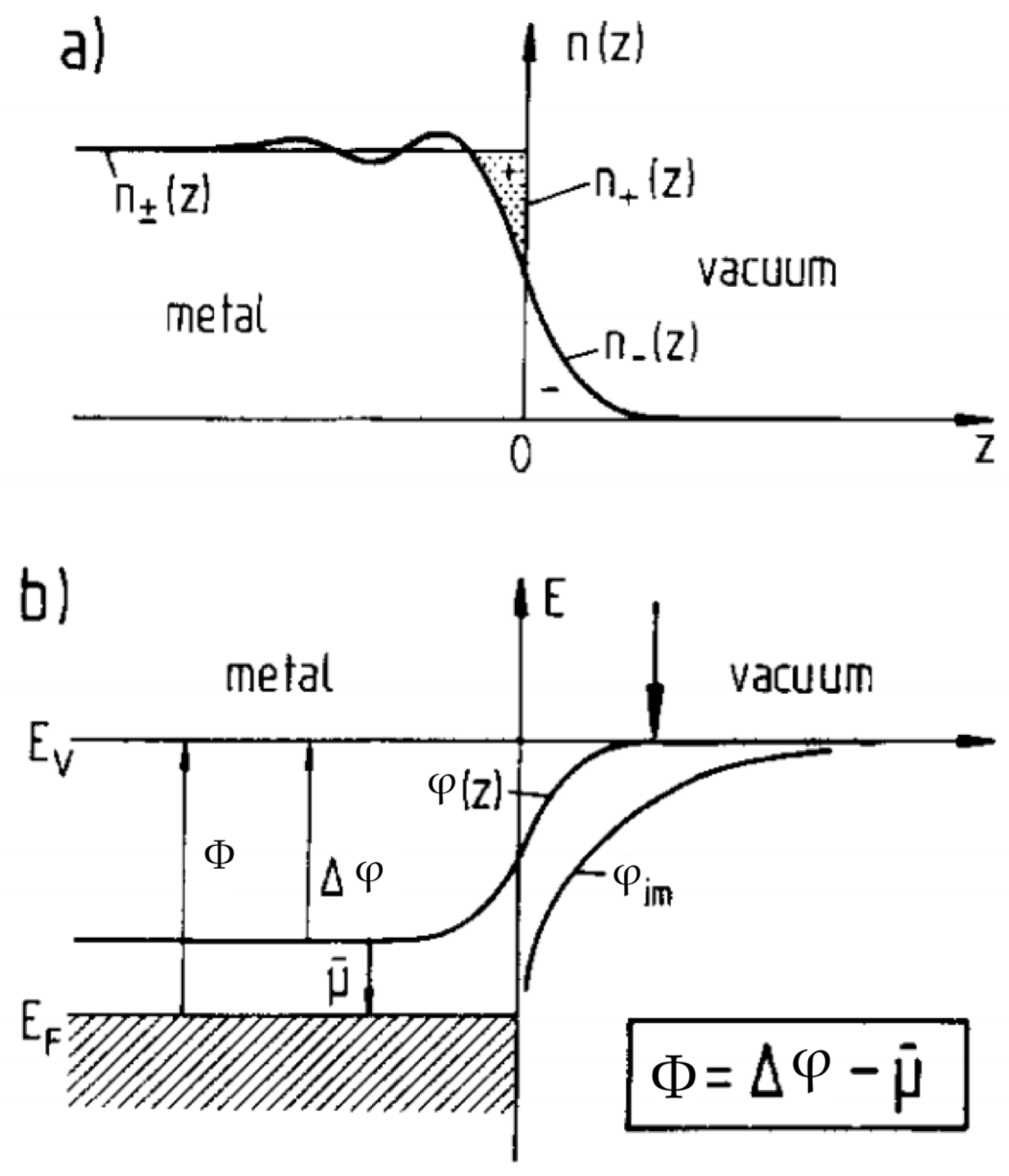

Figure 21 a) Charge density $\mathrm{n}_{ \pm}(\mathrm{z})$ distribution perpendicular to a calculated jellium surface. Negative charge leaking into vacuum and the resulting positive charge within the solid result in the creation of the surface dipole layer. b) Potential energy diagram explaining the components of the work function. $\Delta \varphi$ is the surface dipole barrier, $\bar{\mu}$ is the chemical potential of an electron in the bulk of a metal, $\Phi$ is the work function, $\varphi_{\mathrm{im}}$ is the image potential of an electron close to the surface. The figure has been adapted from Wandelt. ${ }^{78}$ 
If we let the electrostatic potential energy of an electron as a function of distance perpendicular to the surface be given by $\varphi(z)$, we can describe the surface dipole as the potential step:

$$
\Delta \varphi=\varphi(\infty)-\varphi(-\infty)
$$

Thus, the potential energy of an electron at rest in vacuum, $\varphi(\infty)$, is greater than the average potential energy of an electron deep in the bulk, $\varphi(-\infty) . \varphi(\infty)$ is often referred to as the vacuum level. Therefore, $\Phi$, the minimum energy needed to transfer an electron from the bulk to the vacuum level can be written as:

$$
\Phi=\Delta \varphi-\bar{\mu}
$$

From the foregoing discussion, it is obvious how an adsorbate can influence work function. Adsorbates at the surface of a solid cannot influence $\bar{\mu}$, but can have a strong effect on the magnitude of $\Delta \varphi$. Atoms and molecules physisorbed at a metal surface are bound to the surface through attraction between induced dipole in the adsorbate and the dipole's image in the bulk. These dipoles will, depending on the orientation, also attract or repel electron density near the surface, leading to an increase or decrease in the surface dipole, $\Delta \varphi$. This results in a direct modification of the surface work function.

More strongly bound species can have dramatic effects on the work function of metal surfaces beyond simple dipole interaction. For example, the bond of open-shell atoms with metal surfaces can be expected to involve significant charge transfer and have a dramatic effect on a metallic surface work function. For example, adsorbed halogens and alkali metals are known to significantly influence the work function of metal surfaces. ${ }^{79,80}$

\subsubsection{Kelvin probe work function measurements}

I measured the work function of clean and adsorbate covered surfaces using a scanning Kelvin probe (KP technology, UHVKP020). A Kelvin probe is an excellent tool for measuring the work function of adsorbate covered surfaces because it is completely non-destructive and will not induce desorption of even very weakly bound physisorbed particles. The work function measurements reported in this work follow the procedure developed by Baikie, et al. ${ }^{81,82}$ 


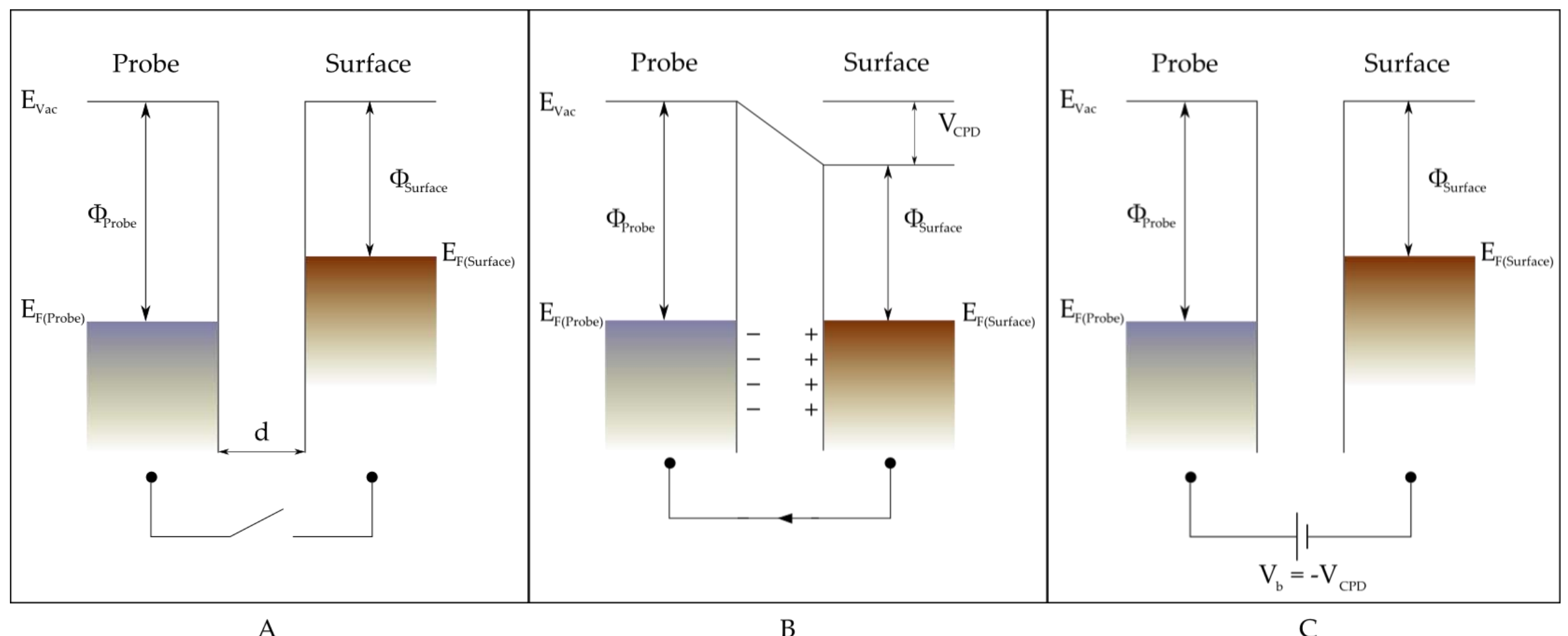

A

B

C

Figure 22 Theory of kelvin probe work functions measurement. Panel A shows the energy level diagram for the conducting probe head and a conducting, isolated sample. $\Phi_{\text {Probe }}$ and $\Phi_{\text {Surface }}$ are the work functions of the two samples. In the case presented, the probe head's work function is greater than that of the surface. If, as shown in panel B, external electric contact is made between the surface and the probe, their potentials equalize and the flow of charge results in the two plates having equal and opposite charge. The current flowing across the external circuit, $I_{\mathrm{c}}$, can be monitored versus a scanned external backing potential, $V_{\mathrm{b}}$ applied to one of the electrodes and the point where $V_{\mathrm{b}}=-V_{\mathrm{CPD}}$ corresponds to $I_{\mathrm{c}}=0$. 
In order to perform an experiment, the surface of interest is maneuvered in front of the reference electrode of the Kelvin probe to form a simple capacitor. As seen in Figure 22, when two surfaces with differing work functions $\left(\Phi_{\text {Probe }}\right.$ and $\left.\Phi_{\text {Surface }}\right)$ are brought close together (left panel) and connected via an electronic circuit (center panel), charge flows from the low work function surface to the high work function surface resulting in a potential gradient. This is the contact potential difference voltage $\left(V_{\mathrm{CPD}}\right)$, between the two surfaces. By introducing a variable backing potential, $V_{b}$, in the external circuit, one electrode can be biased against the other. By monitoring the current between the plates while scanning $V_{\mathrm{b}}$, the unique point where $V_{\mathrm{b}}=-V_{\mathrm{CPD}}$ results in a null current. This voltage is the work function difference between the two surfaces.

This method allows a once-only measurement as the surfaces become charged and this charge must dissipate before another measurement can be made. Additionally, the accuracy of the measurement is limited because signal diminishes with respect to background as null voltage is approached.

These problems can be overcome by vibrating the probe head in front of the surface to be measured, i.e. varying $d$ with a constant $V_{\mathrm{b}}$. The tip and sample separated by vacuum have a capacitance:

$$
C=\varepsilon_{0} \frac{A}{d}
$$

where $A$ is the capacitative surface area, $d$ is the probe-sample distance, and $\varepsilon_{0}$ is the permittivity of vacuum. Alternatively, capacitance can be written as:

$$
C=\frac{q}{V}
$$

where $q$ is charge. Therefore, as the separation between probe and sample increases, capacitance decreases. As the charge remains constant, the voltage $V$ must increase. Relating these two expressions for capacitance gives the relation:

$$
V \propto d
$$

A change in $d$ results in a change in $V$. 
By vibrating the probe head position in front of the surface in a sinusoidal fashion at a given $V_{\mathrm{b}} \neq-V_{\mathrm{CPD}}$ and monitoring the voltage difference between probe and surface, we see a periodic signal such as that shown in Figure 23.

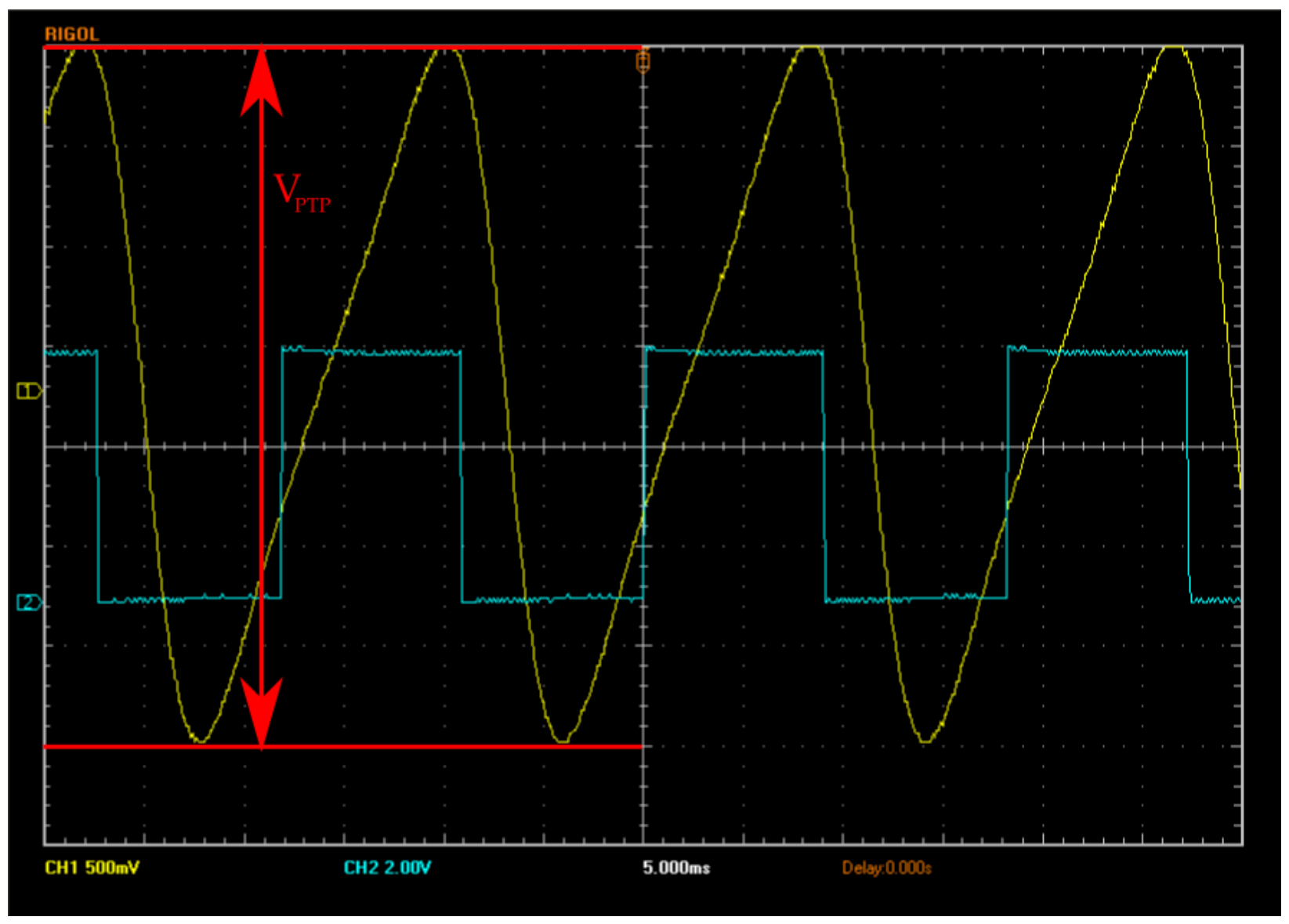

Figure 23 Raw signal of voltage between probe and sample while vibrating Kelvin probe head in front of sample surface at constant $V_{\mathrm{b}} \neq-V_{\mathrm{CPD}}$. By plotting peak-to-peak voltage, $V_{\mathrm{PTP}}$ versus $V_{b}$ one can accurately determine the contact potential difference between probe and sample.

When $V_{\mathrm{b}}$ cancels out $V_{\mathrm{CPD}}$, the charge on tip and sample are equal and varying sample distance does not result in a time varying raw signal voltage. Calculating peak-to-peak voltage $V_{\mathrm{PTP}}$ for many values of $V_{\mathrm{b}}$ and plotting $V_{\mathrm{PTP}} v s . V_{\mathrm{b}}$ allows one to determine the work function difference between probe and sample with great accuracy with minimal effect of electronic noise. 


\subsubsection{Scattering and detection}

$\mathrm{CO}^{*}$ is detected in the apparatus via three different methods. First, we can detect the phosphorescence of $\mathrm{CO}^{*}$ using a photomultiplier tube (PMT). Secondly, when $\mathrm{CO} *$ impinges on a metal surface, electrons are emitted which we detect on a microchannel plate (MCP). Thirdly, the incident molecules can be ionized with $283 \mathrm{~nm}$ laser radiation and the ions can be subsequently extracted onto a second MCP.

\subsubsection{Photomultiplier tube detection}

Since CO* decays spontaneously to the ground electronic state, emitted photons can be detected with a PMT. Because decay of $\mathrm{CO}^{*}$ to the ground state can only occur via a spinforbidden transition, the molecule exhibits a long lifetime of $2.63 \mathrm{~ms} .{ }^{39}$ This type of detection is inefficient as only a small fraction of the molecular beam phosphoresces within the area observable to the PMT. The advantage, however, is that because the decay is spontaneous, one can observe the intensity of the phosphorescence signal on the PMT as it passes through the chamber without changing the quality of the beam to be scattered. In this way, the detection can be thought of as "non-destructive."

The intensity of the phosphorescence signal collected on the PMT as the molecular beam passes by is proportional to the number of excited molecules in the beam pulse after laser excitation. The PMT signal is therefore a good first step in optimizing the molecular beam/laser overlap and laser wavelength of our initial electronic excitation step using the $206 \mathrm{~nm}$ laser. Because phosphorescence is measured upstream from the decelerator and not every CO* molecule in the pulse couples into the decelerator, one cannot assume that larger PMT signal translates in all cases into more molecules making it through the decelerator, but in general more CO* signal recorded on the PMT correlates with a stronger signal measured after the decelerator.

In order to use PMT detection, we have mounted a head-on, $15 \mathrm{~mm}$ active area solar blind PMT (Hamamatsu Photonics R821, $3.6 \times 10^{5}$ gain) perpendicular to the beam axis immediately outside the chamber, $150 \mathrm{~mm}$ from the beam axis, $23.3 \mathrm{~mm}$ downstream from the differential pumping wall between hexapole and decelerator, see Figure 3. 


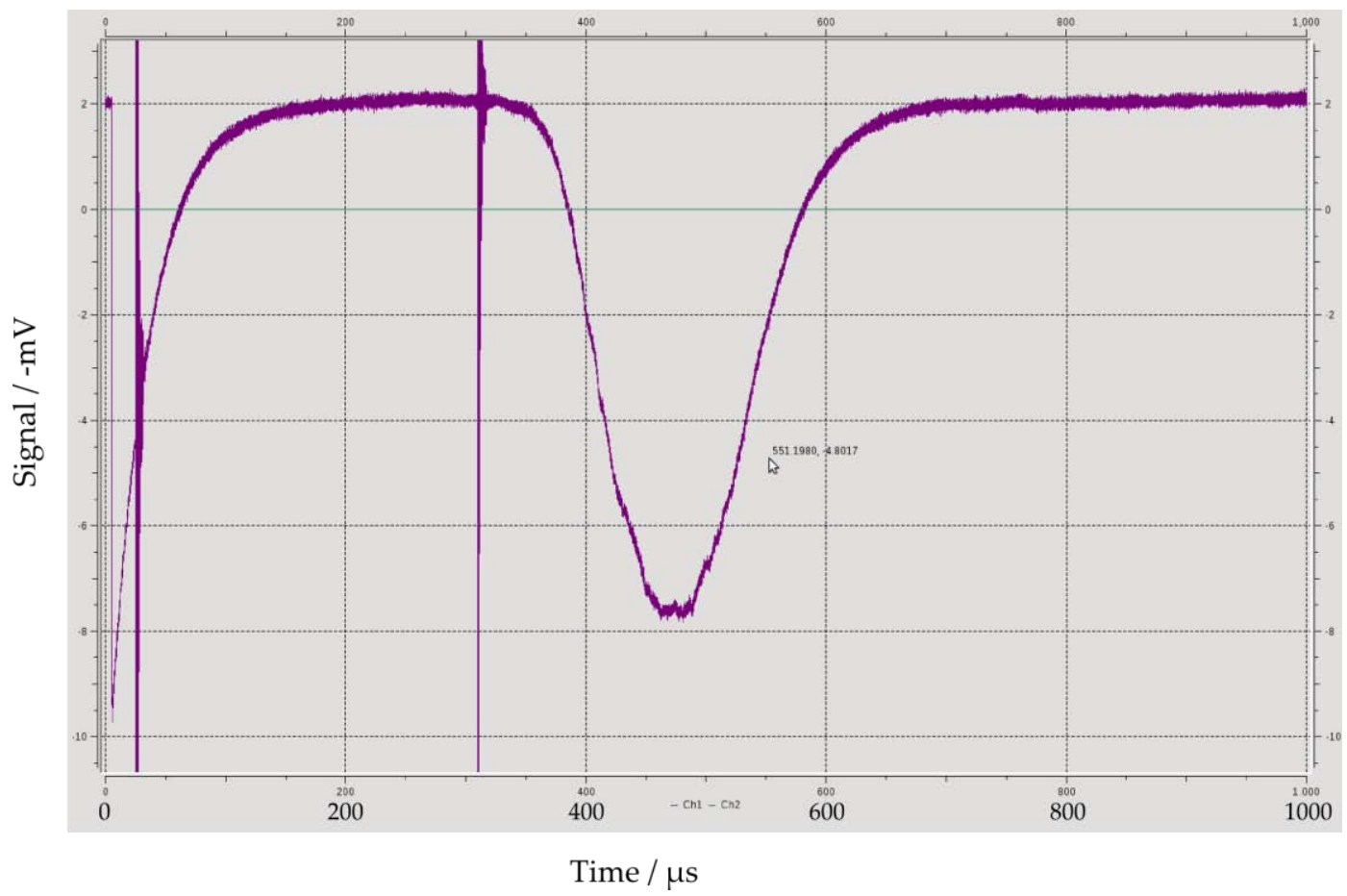

Figure 24 Typical signal of $206 \mathrm{~nm}$ photons incident on PMT. The initial quickly decaying signal at time $t=0 \mu \mathrm{s}$ is the $206 \mathrm{~nm}$ laser pulse used to prepare CO*, the broad peak centered around time $t=475 \mu \mathrm{s}$ is the phosphorescence of the beam pulse after having been focused around the $3.5^{\circ}$ angle through the hexapole and into the decelerator chamber. The sharp pulses at $t=25 \mu \mathrm{s}$ and $310 \mu \mathrm{s}$ are electrical pickup from the hexapole switching on and off.

\subsubsection{REMPI ionization and charged particle detection}

We use resonance enhanced multiphoton ionization (REMPI) spectroscopy to probe the molecular beam after it has left the decelerator and before it scatters from the surface. REMPI can also be used to detect scattered molecules with quantum state sensitivity. $\mathrm{CO}^{*}$ molecules are detected prior to surface collision by 1+1 REMPI using a frequency doubled pulsed dye laser (Spectra Physics PDL-2, pumped by the second harmonic of a Continuum Powerlite 9010 Nd:YAG laser) resonant at $283 \mathrm{~nm}$ with the $\mathrm{b}^{3} \Sigma^{+} \leftarrow \mathrm{a}^{3} \Pi_{1}$ transition. The ions are then extracted toward a home built detector consisting of a double MCP stack in a chevron configuration and an extraction grid (91\% transmission, $\mathrm{Ni}$ ) mounted in the chamber $8 \mathrm{~cm}$ below the molecular beam axis. The MCP stack and extraction grid are powered by individual power supplies (iseg Spezialelektronik GmbH EHQ 105). By controlling the voltage on the extraction grids independently from the detector voltage, we can vary the extraction conditions to optimize 
collection under different experimental conditions while retaining MCP gain conditions. This facilitates much simpler analysis when performing comparative measurements.

Mounted $4 \mathrm{~cm}$ above the molecular beam axis, parallel to the MCP detector described above, is a second MCP detector of similar design. The second detector is used to detect electrons ejected when $\mathrm{CO}^{*}$ is quenched at the surface. ${ }^{83}$ By characterizing the electron emission from this process from atomically clean $\mathrm{Au}(111)$, we can monitor the population of the molecular beam pulses. Further, we can use this knowledge to determine dumping efficiencies when preparing ground state $\mathrm{CO}$ for surface scattering, and we have a convenient benchmark for comparison of electron emission probability with different surfaces.

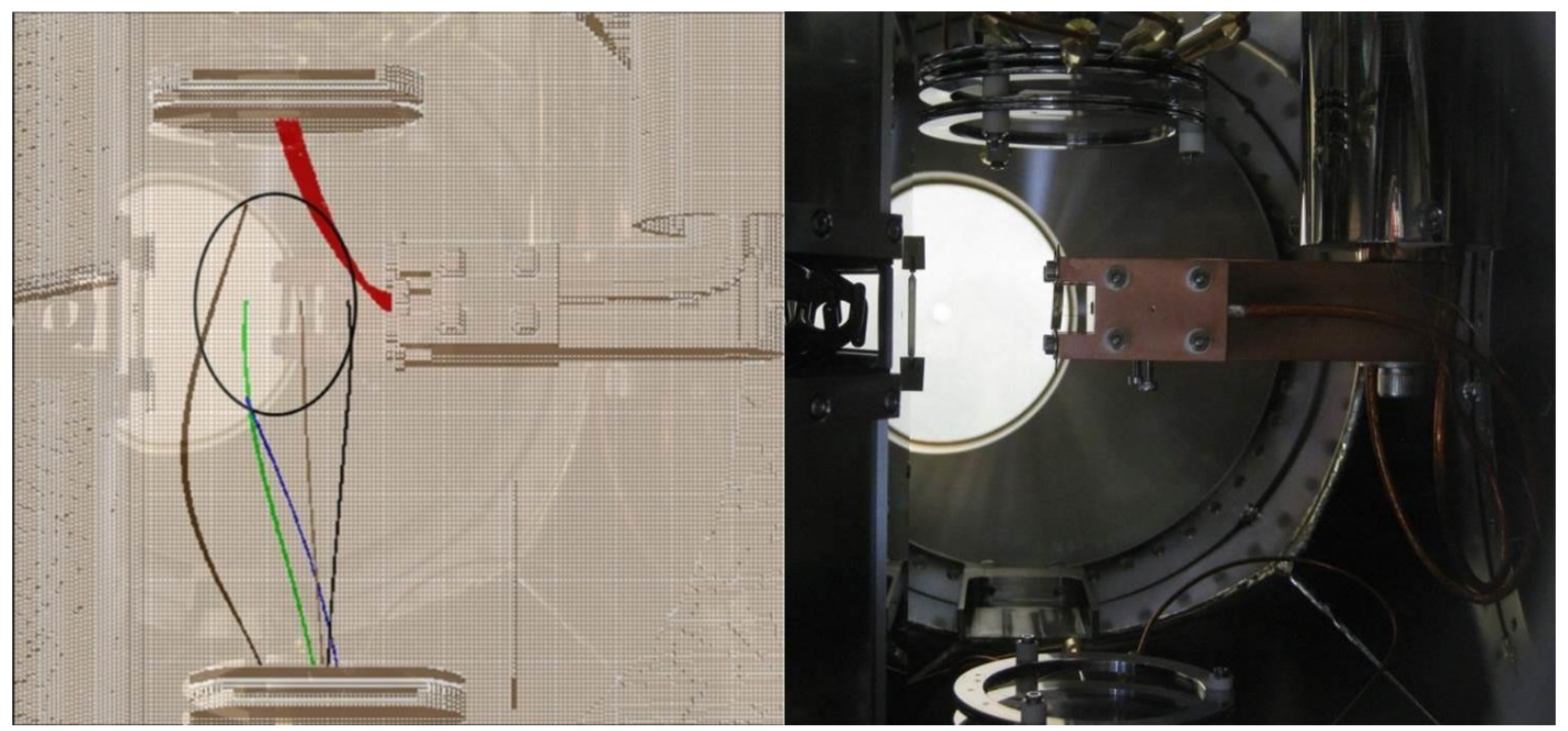

Figure 25 Right panel: photograph of surface position in front of molecular beam. MCP detectors are mounted above and below the scattering region. Left panel: SimION simulation of electrons originating from the surface (red), $\mathrm{CO}$ in incident molecular beam pulse at 10, 20, and $30 \mathrm{~mm}$ from the surface (black, tan, green) and molecules scattered at $35^{\circ}$ relative to front plane of the $\mathrm{Au}(111)$ crystal (brown, blue). The black ellipse indicates the large region in which ionized particles can be extracted with $100 \%$ efficiency.

Shown in the right panel of Figure 25 is a photograph of the sample holder, charged particle detectors and differential pumping wall separating the decelerator from the surface 
chamber. The panel on the left is a SimION (Scientific Instrument Services, Inc.) trajectory simulation of charged particles originating at various positions relative to surface and detector. Red trajectories are those of electrons originating near the surface and all others are ionic trajectories. Initial conditions for the ions are mass of $28 \mathrm{amu}$ traveling either toward the surface for those along the molecular beam axis (green, grey, black) or away from the surface for those off the beam axis (brown, blue) with $151.66 \mathrm{~cm}^{-1}(360 \mathrm{~m} / \mathrm{s}$ ) of translational energy. The ions originating off axis represent molecules scattered from the surface. 100\% extraction is possible for a wide range of geometries, indicated in Figure 25 with the black ellipse, allowing for efficient time-of-flight and angular distribution measurements. Scanning the ionization laser can be accomplished by means of a periscope outside the chamber. Laser access into the surface chamber is accomplished via $100 \mathrm{~mm}$ UV fused silica windows mounted on each side of the surface scattering region.

As can be seen in the photograph, laser access to the decelerator chamber has been made possible by mounting a special end cap on the surface side of the decelerator chamber which extends into the surface chamber. Two UV fused silica windows are installed at Brewster's angle on either side of the cap to allow laser access to the molecular beam for six electrode stages before the end of the decelerator. This allows us to shine a laser between the deceleration stages to dump molecules from the electronically excited state to a chosen ro-vibrational level in the electronic ground state via stimulated emission before the pulse has left the decelerator. Simulations indicate that last decelerator stages can deflect the remaining metastables off the beam axis in order to prepare a beam of pure electronic ground state molecules for scattering from the surface. 


\section{Experimental results}

\subsection{TPD of weakly bound adsorbates on $\mathrm{Au}(111)$}

As will be presented in section 3.2.4, dependence of electron emission on the surfacemolecule distance has been directly probed by scattering $\mathrm{CO}^{*}$ from $\mathrm{Au}(111)$ prepared with controlled adsorbate coverages. Surface-molecule approach distance can be limited by the careful adsorption of non-reactive "spacer layers" of noble gases. As noble gases are closed-shell species and chemically inert, the minimum distance of approach for a molecule approaching a metal surface covered with a monolayer of rare gas adsorbate can be approximated from the van der Waals radius of the adsorbate gas.

In order to perform these experiments with precise knowledge of surface coverage, temperature programmed desorption (TPD) experiments were first performed in order to characterize adsorbate coverage as a function of adsorbate exposure and surface temperature. The TPD results were also analyzed to determine the desorption activation energy $\left(E_{\mathrm{des}}\right)$ of a number of different atomic and molecular adsorbates. It is shown that $E_{\text {des }}$ is an excellent proxy for the binding energy adsorbates within this temperature regime and therefore these experimental results will serve as benchmarks for theory which describes dispersion force interactions of molecules at metal surfaces.

\subsubsection{Temperature programmed desorption}

In addition to TPD studies of the rare gases $\mathrm{Ar}, \mathrm{Kr}$, and $\mathrm{Xe}$ on $\mathrm{Au}(111)$, this chapter will present the results of TPD experiments involving the molecular adsorbates $\mathrm{C}_{2} \mathrm{H}_{2}, \mathrm{SF}_{6}, \mathrm{~N}_{2}, \mathrm{NO}$ and $\mathrm{CO}$ on $\mathrm{Au}(111)$. While these more complex adsorbates cannot be considered unreactive spacer layers, studies of molecular desorption kinetics provide important benchmarks for theory and also lay the groundwork for scattering molecules from more complex surfaces.

One of the most important challenges for ab initio theory is an accurate treatment of dispersion forces. Potential energy surfaces (PES) of interfacial systems are generally calculated using density functional theory (DFT) as it can be applied to both molecules and surfaces comprising thousands of atoms. Long range van der Waal's forces, however, are often not accurately described within the DFT framework. Recent theoretical developments to accurately 
represent these forces in the DFT approach ${ }^{84-87}$ must be compared with accurate experimental data in order to test their validity. The energetics of molecules and atoms at surfaces provide convenient reference data for emerging theory. The desorption activation energies $\left(E_{\text {des }}\right)$ reported in this work represent important benchmarks against which newly developing theories will be tested. ${ }^{88}$

The $\mathrm{Au}(111)$ surface is particularly important as a theoretical benchmark as it is used in many model systems to test molecular dynamics on chemically inactive surfaces. ${ }^{22,89-91}$ Due to gold's energetically low-lying $d$-orbitals, the interaction between adsorbates and surfaces is usually rather weak. This phenomenon allows state resolved studies of energy transfer between molecules and surfaces using molecular beams without competing surface reactions. For example, studies on energy transfer between incident molecular degrees of freedom and electronhole pairs have yielded many exciting discoveries in recent years. ${ }^{24,27,30,83,90,92-96}$ In particular, clear evidence of non-adiabatic energy transfer has been demonstrated using experiments which scatter vibrationally excited nitric oxide from the $\mathrm{Au}(111)$ surface, including electron transfer to the molecule. ${ }^{24,26,27,30,32,91,92,95-97}$

Because $\mathrm{Au}(111)$ is chemically unreactive, however, very little experimental data exists on the binding properties of adsorbates on the $\mathrm{Au}(111)$ surface. The desorption activation energy of atoms and small molecules on $\mathrm{Au}(111)$ is a useful tool for interpreting more complicated experimental results. In order to construct and test the validity of quantum chemical simulations of surface dynamics, detailed knowledge of $\mathrm{Au}(111)$ properties is necessary. ${ }^{32,98}$ A reliable PES is the key of any ab initio theory describing surface dynamics. Erroneous PESs lead to incorrect results.

\subsubsection{Experimental and results}

A convenient method for determining $E_{\text {des }}$ of adsorbed molecules on surfaces is temperature programmed desorption. The complete experimental setup including adsorbate doser and shielded quadrupole mass spectrometer is described in detail in section 2.2.2, as well as the experimental procedure and details of the analysis. Briefly, the procedure involves dosing a cold surface with the gas of interest, then heating the dosed surface at a controlled rate, $k$, while monitoring the partial pressure of the adsorbed species with an ion gauge or a mass spectrometer. Ion gauges have the advantage of extremely high ionization efficiency, but this efficiency is 
outweighed by the ability of a mass spectrometer to detect gas phase species with mass resolution. A quadrupole mass spectrometer was used for all studies in this work. When pumping speed is fast enough that essentially no re-adsorption takes place, the partial pressure of the desorbing species is directly proportional to the desorption rate. Rate of desorption increase as surface temperature, $T_{\mathrm{S}}$, rises. The partial pressure signal increases with time (and surface temperature) until the population of adsorbed particles has been depleted. The partial pressure then drops rapidly, resulting in a peak on a plot of partial pressure versus $T_{\mathrm{S}}$. Particles in each different bound state on the same surface will exhibit different characteristics. For instance, a particle bound directly to the surface (monolayer) will often bind more strongly than particles bound immediately atop the monolayer (bilayer). The different desorption characteristics result in distinct peaks for each adsorbate binding state.

Shown in Figure 26 are TPD traces of the noble gases $\mathrm{Ar}, \mathrm{Kr}$, and $\mathrm{Xe}$ in panels 1-3 and the molecules $\mathrm{C}_{2} \mathrm{H}_{2}, \mathrm{SF}_{6}, \mathrm{~N}_{2}, \mathrm{NO}$ and $\mathrm{CO}$ in panels 4-8. All traces are taken at $k=0.5 \mathrm{~K} / \mathrm{s}$ with initial adsorbate coverage $\theta_{\mathrm{i}}$ of around 3 monolayers (ML). The TPD spectra of $\mathrm{Ar}, \mathrm{Kr}$, and Xe are qualitatively quite similar. Each exhibits well separated monolayers at 45, 63, and $90 \mathrm{~K}$, respectively, each labeled $\alpha$. The monolayer line shape is indicative of zeroth order desorption kinetics. This interpretation of the line shape is consistent with previous measurements of rare gas/metal systems. ${ }^{99}$ The rare gas desorption spectra each show a clear bilayer peak at 33, 46, and $63 \mathrm{~K}$ for $\mathrm{Ar}, \mathrm{Kr}$, and $\mathrm{Xe}$, respectively, designated $\beta$. The $\beta$ peak is saturable for all noble gases desorbing from $\mathrm{Au}(111)$. The peaks labeled $\gamma$ appear at the lowest temperature and are not saturable. This behavior suggests rare gas overlayers that are essentially rare gas ice. 


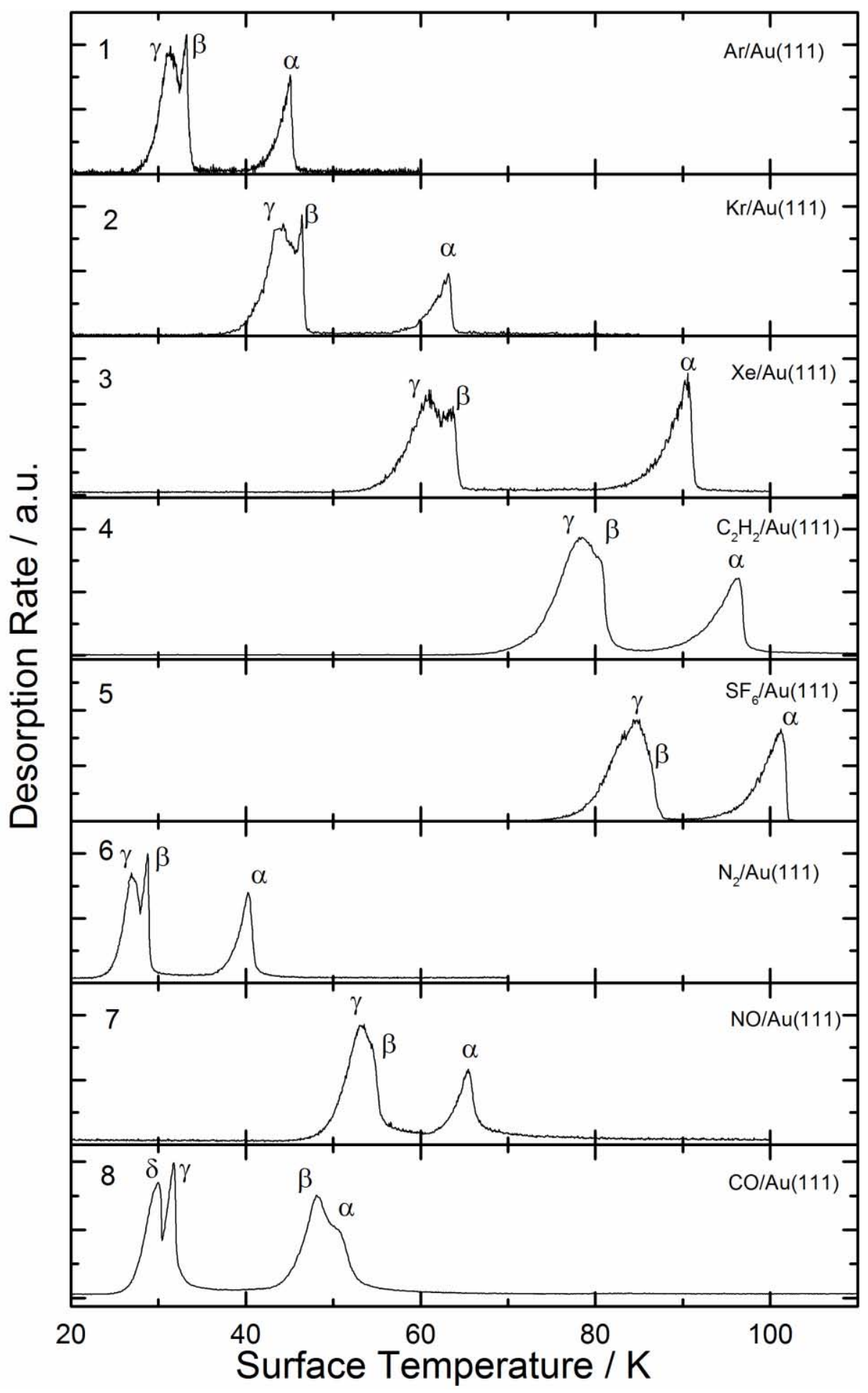

Figure 26 Thermal desorption spectra for $\mathrm{Ar}, \mathrm{Kr}, \mathrm{Xe}, \mathrm{C}_{2} \mathrm{H}_{2}$ and $\mathrm{SF}_{6}, \mathrm{~N}_{2}, \mathrm{NO}$, and $\mathrm{CO}$. All traces are recorded at $k=0.5 \mathrm{~K} / \mathrm{s}$. See text for discussion. 
The TPD spectra of $\mathrm{C}_{2} \mathrm{H}_{2}$ and $\mathrm{SF}_{6}$ are shown in panels 4 and 5. Both are qualitatively similar to the rare gas TPD traces. Each shows a monolayer peak, $\alpha$ - at $96 \mathrm{~K}$ for $\mathrm{C}_{2} \mathrm{H}_{2}$ and 101 $\mathrm{K}$ for $\mathrm{SF}_{6}$ - that is well separated from the bilayer peak, $\beta$, which is visible in both traces as a shoulder on the high temperature side of the non-saturable overlayer peak, $\gamma$. Line shape analysis of the monolayer peak indicates zeroth order desorption kinetics. Zeroth order desorption kinetics suggest that two dimensional island formation occurs at high initial coverages due to lateral attraction between adsorbate atoms. Desorption rates are independent of coverage because desorption takes place preferentially from island edges. ${ }^{74}$

The characteristic asymmetric monolayer line shapes in the TPD traces of $\mathrm{N}_{2}$ and NO, shown in panels 6 and 7, indicate first order desorption processes. The monolayer peak maxima, labeled $\alpha$, appear at $40 \mathrm{~K}$ and $65 \mathrm{~K}$ for $\mathrm{N}_{2}$ and $\mathrm{NO}$, respectively. The bilayer peak maximum, $\beta$, can be clearly distinguished from the non-saturable overlayer peak in the $\mathrm{N}_{2}$ trace, while the NO bilayer is visible as a shoulder on the high temperature side of the overlayer peak.

Mullins and co-workers observed an NO desorption peak from $\mathrm{Au}(111)$ which peaked around $150 \mathrm{~K} .{ }^{100}$ This result differs from the work of Bartram and Koel, who found no evidence of $\mathrm{NO}$ desorption from $\mathrm{Au}(111)$ down to $95 \mathrm{~K}$. This high temperature desorption peak was attributed by Mullins to nitric oxide adsorbing at defect sites on their crystal, and suggested that the differences between spectra could be attributed to different concentrations of defects for different crystals. The data reported in this work demonstrates unambiguously that stable adsorption of $\mathrm{NO}$ on $\mathrm{Au}(111)$ only occurs below $70 \mathrm{~K}$, confirming the work of Koel and suggesting that Mullins's interpretation of his results is plausible.

The monolayer peak in the thermal desorption spectrum of $\mathrm{CO}$ (panel 8) shows two overlapping peaks. The larger peak, $\beta$, is seen at $47 \mathrm{~K}$ and the smaller, $\alpha$, at $50 \mathrm{~K}$. This double peak structure is assigned to the relaxation of a densely packed structure which is formed as the monolayer is nearly complete. This so-called decompression peak has been observed in the case of $\mathrm{CO}$ desorption from $\mathrm{Au}(110)$ at similar temperatures. The $\mathrm{CO}$ monolayer in the densely packed phase, $\beta$, are oriented more perpendicularly to the surface than in the less densely packed phase assigned to the $\alpha$ peak. 
More detailed TPD data for each species are presented in Figures 27-34. For each gas, coverage has been systematically varied. Argon (Figure 27), Krypton (Figure 28), Xenon (Figure 29), $\mathrm{C}_{2} \mathrm{H}_{2}$ (Figure 30), and $\mathrm{SF}_{6}$ (Figure 31) desorb following zeroth order kinetics, as indicated by the common leading edge for all coverages and the shift of peak maximum to lower temperature with decreasing coverage. Figure 32 shows thermal desorption spectra for different initial doses of $\mathrm{N}_{2}$. The peak maximum remains constant for different initial coverages and no common leading edge is observed, indicating first order desorption kinetics. TPD of fractional coverages of NO also indicates first order desorption kinetics and can be seen in Figure 33.

Figure 34 displays TPD spectra for CO with systematically varied initial coverages. At higher coverages, two peaks can be clearly distinguished. While the $\alpha$ peak is populated at low coverages, the $\beta$ peak is formed only when the monolayer approaches saturation. These observations are consistent with the hypothesis of a coverage dependent phase transition. Both peak maxima remain constant with varying initial adsorbate coverages, indicating first order desorption kinetics. 


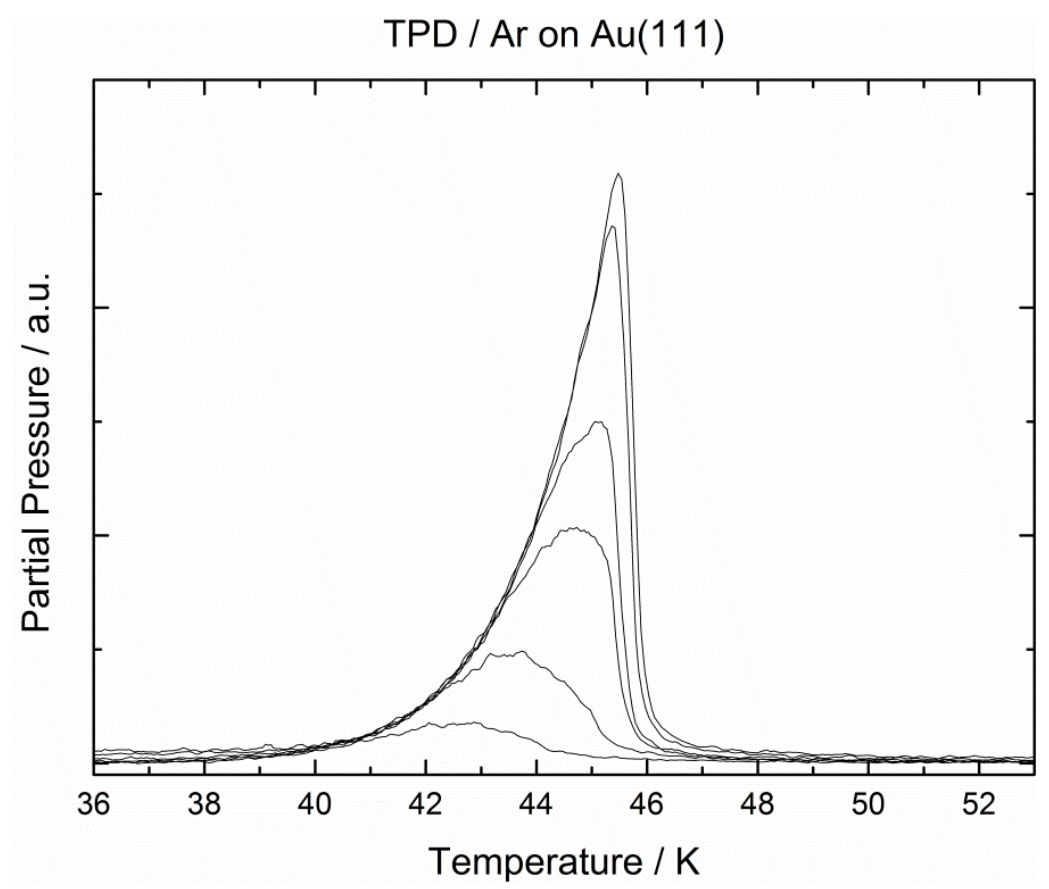

Figure 27 TPD series for sub-monolayer coverages of $\mathrm{Ar}$ adsorbed on $\mathrm{Au}(111)$ taken with heating rates of $k=0.5 \mathrm{~K} / \mathrm{s}$. The spectra correspond to the following initial coverages (in ML): $1.0,0.98,0.84,0.69,0.38,0.17$. The argon peak line shape is typical for zeroth order desorption kinetics

$\mathrm{TPD} / \mathrm{Kr}$ on $\mathrm{Au}(111)$

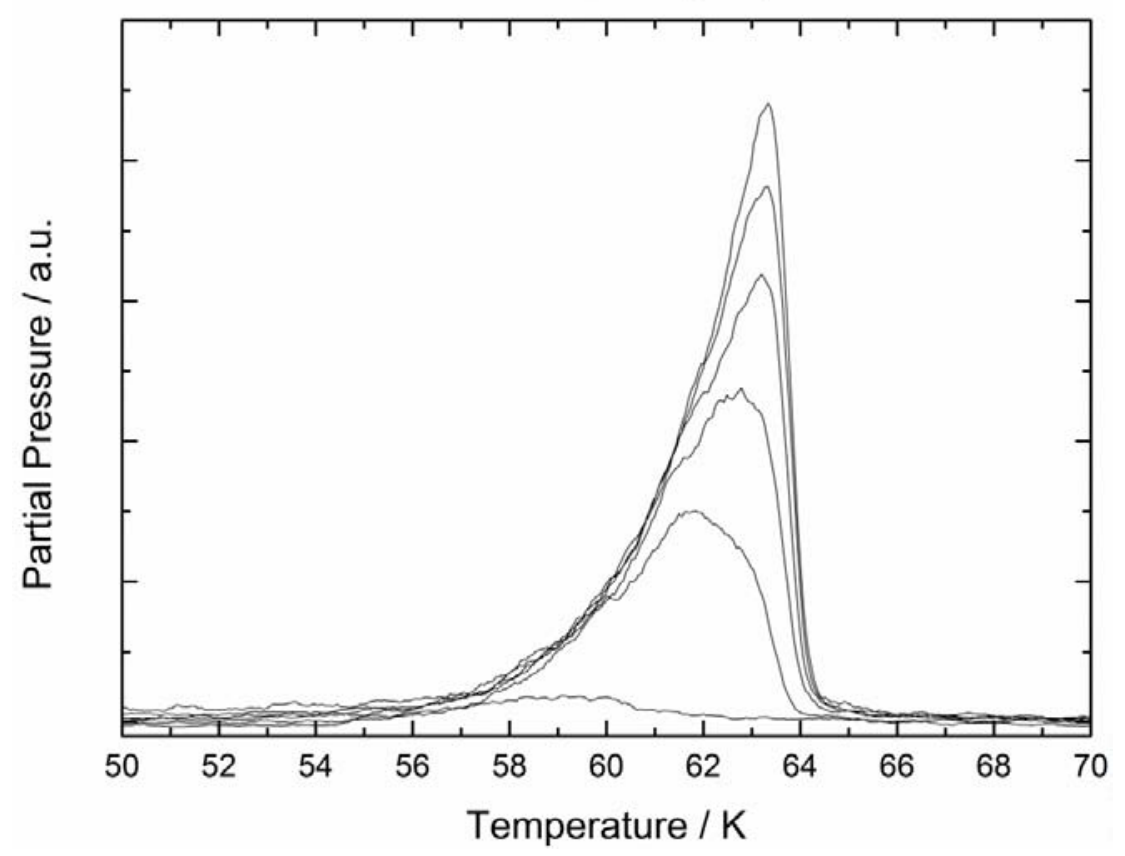

Figure 28 TPD series for sub-monolayer coverages of $\mathrm{Kr}$ adsorbed on $\mathrm{Au}(111)$ taken with heating rates of $k=0.5 \mathrm{~K} / \mathrm{s}$. The spectra correspond to initial coverages (in ML): 1.0, 0.90, 0.76, $0.55,0.4,0.09$. The krypton peak line shape is typical for zeroth order desorption kinetics 
TPD / Xe on Au(111)

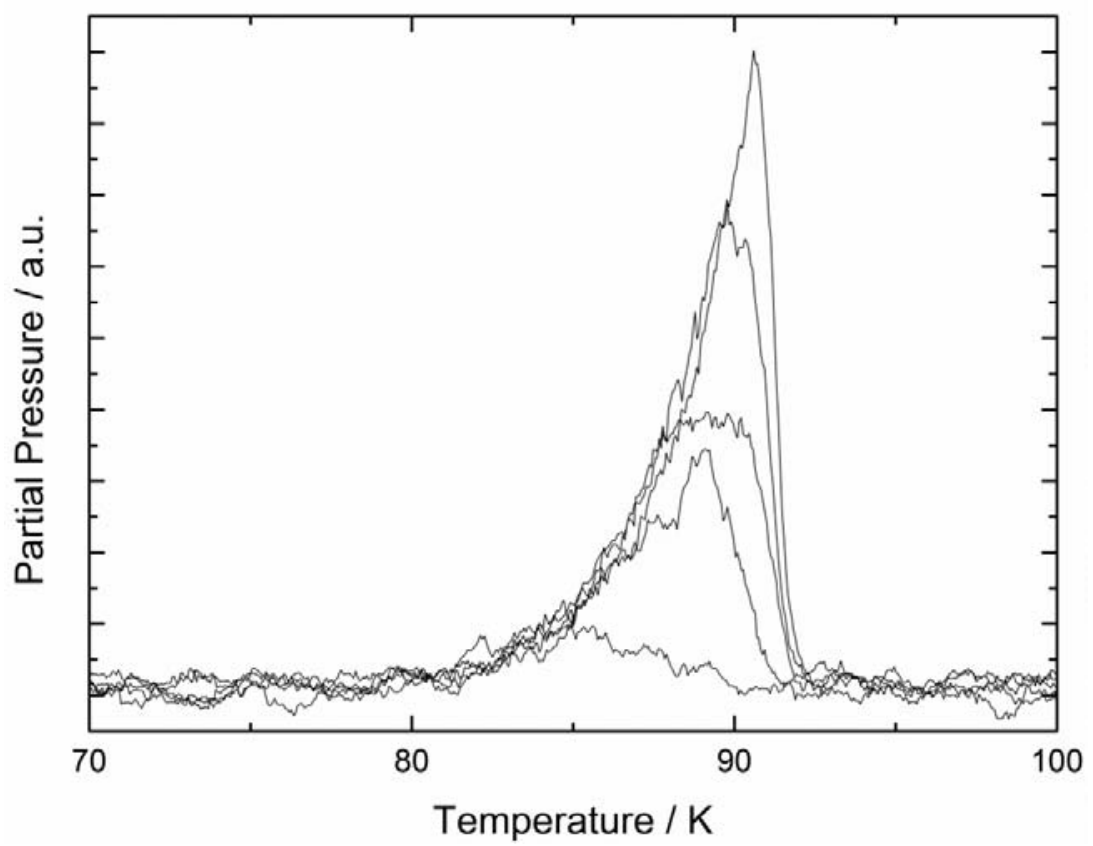

Figure 29 TPD series for sub-monolayer coverages of Xe adsorbed on $\mathrm{Au}(111)$ taken with heating rates of $k=0.5 \mathrm{~K} / \mathrm{s}$. The spectra correspond to initial coverages (in ML): 1.0, 0.85, 0.71, $0.42,0.15$. The xenon peak line shape is typical for zeroth order desorption kinetics

TPD / Acetylene on $\mathrm{Au}(111)$

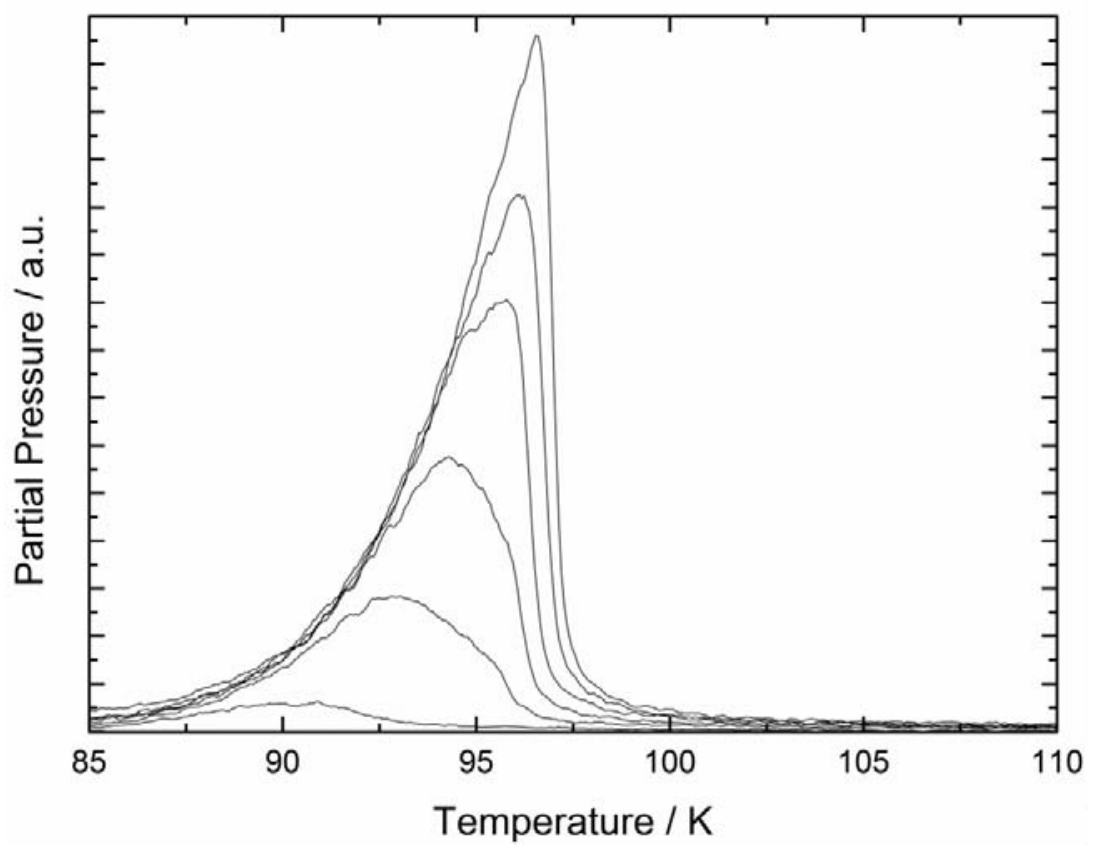

Figure 30 TPD series for sub-monolayer coverages of $\mathrm{C}_{2} \mathrm{H}_{2}$ adsorbed on $\mathrm{Au}(111)$ taken with heating rates of $k=0.5 \mathrm{~K} / \mathrm{s}$. The spectra correspond to initial coverages (in ML): 1.0, 0.89, 0.76, $0.54,0.29,0.04$. The $\mathrm{C}_{2} \mathrm{H}_{2}$ peak line shape is typical for zeroth order desorption kinetics 


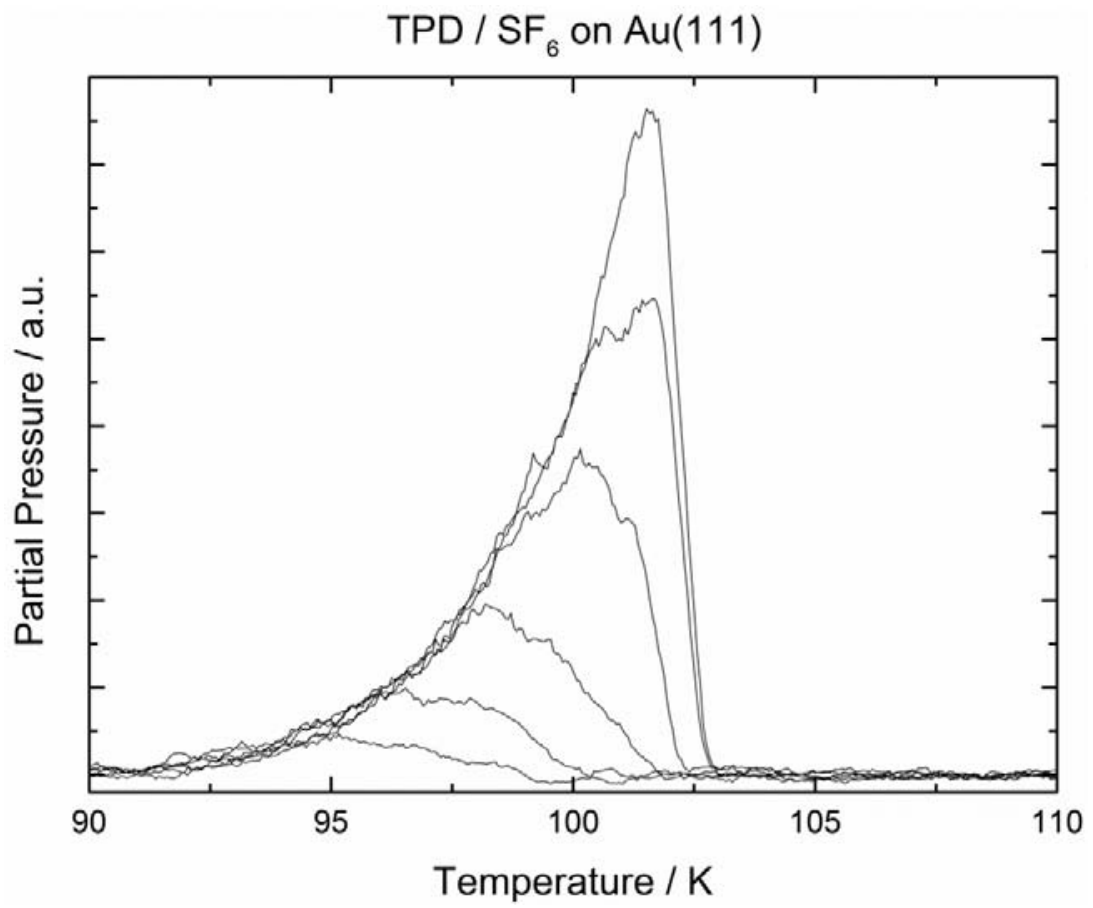

Figure 31 TPD series for sub-monolayer coverages of $\mathrm{SF}_{6}$ adsorbed on $\mathrm{Au}(111)$ taken with heating rates of $k=0.5 \mathrm{~K} / \mathrm{s}$. The spectra correspond to initial coverages (in ML): $1.0,0.87,0.62$, $0.28,0.15,0.08$. The $\mathrm{SF}_{6}$ peak line shape is typical for zeroth order desorption kinetics

TPD / $\mathrm{N}_{2}$ on $\mathrm{Au}(111)$

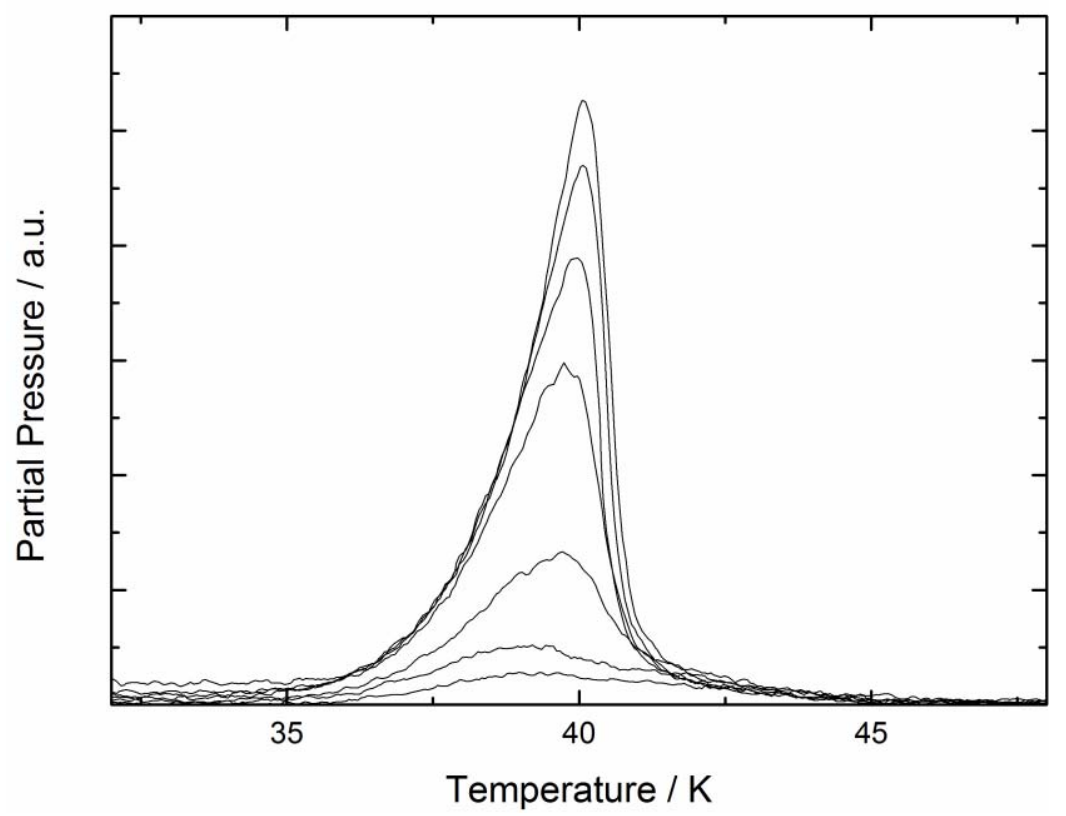

Figure 32 TPD series for sub-monolayer coverages of $\mathrm{N}_{2}$ adsorbed on $\mathrm{Au}(111)$ taken with heating rates of $k=0.5 \mathrm{~K} / \mathrm{s}$. The spectra correspond to the following initial coverages (in ML): $1.0,0.96,0.87,0.76,0.46,0.24,0.15$. 
TPD / NO on Au(111)

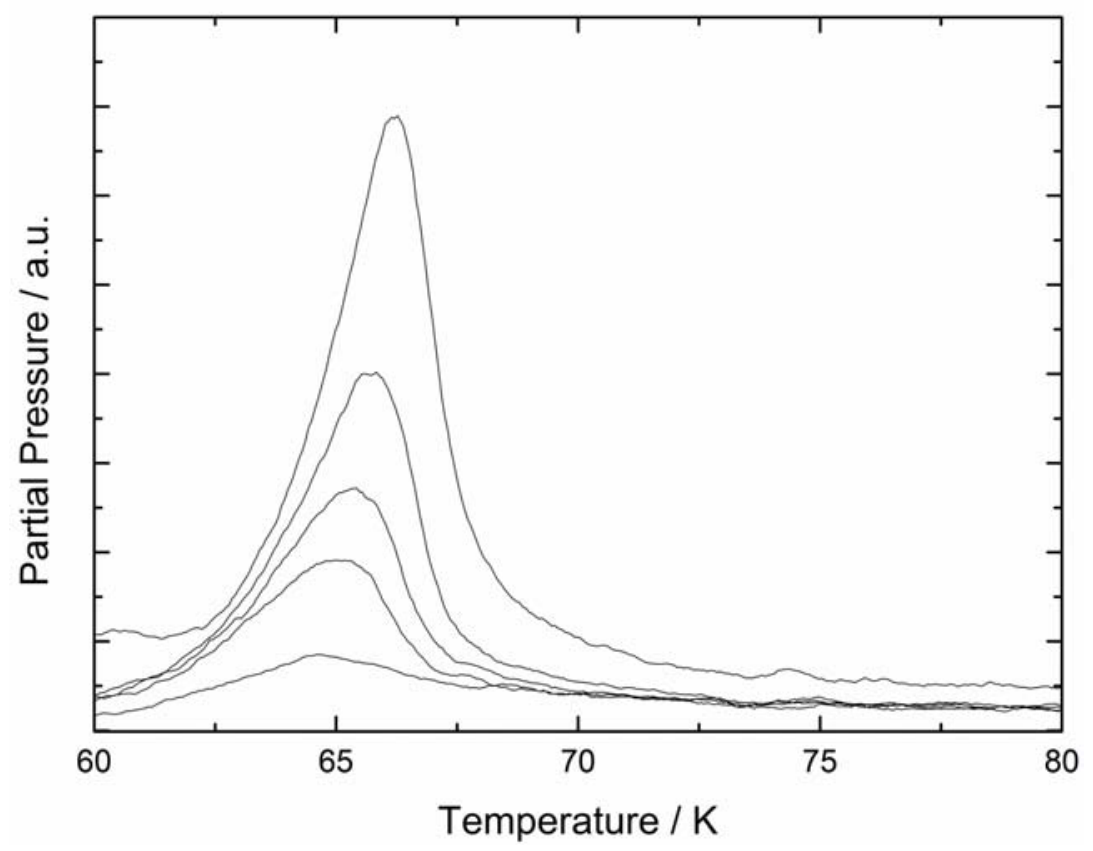

Figure 33 TPD series for sub-monolayer coverages of NO adsorbed on $\mathrm{Au}(111)$ taken with heating rates of $k=0.5 \mathrm{~K} / \mathrm{s}$. The spectra correspond to the following initial coverages (in ML): $1.0,0.62,0.43,0.32,0.013$.

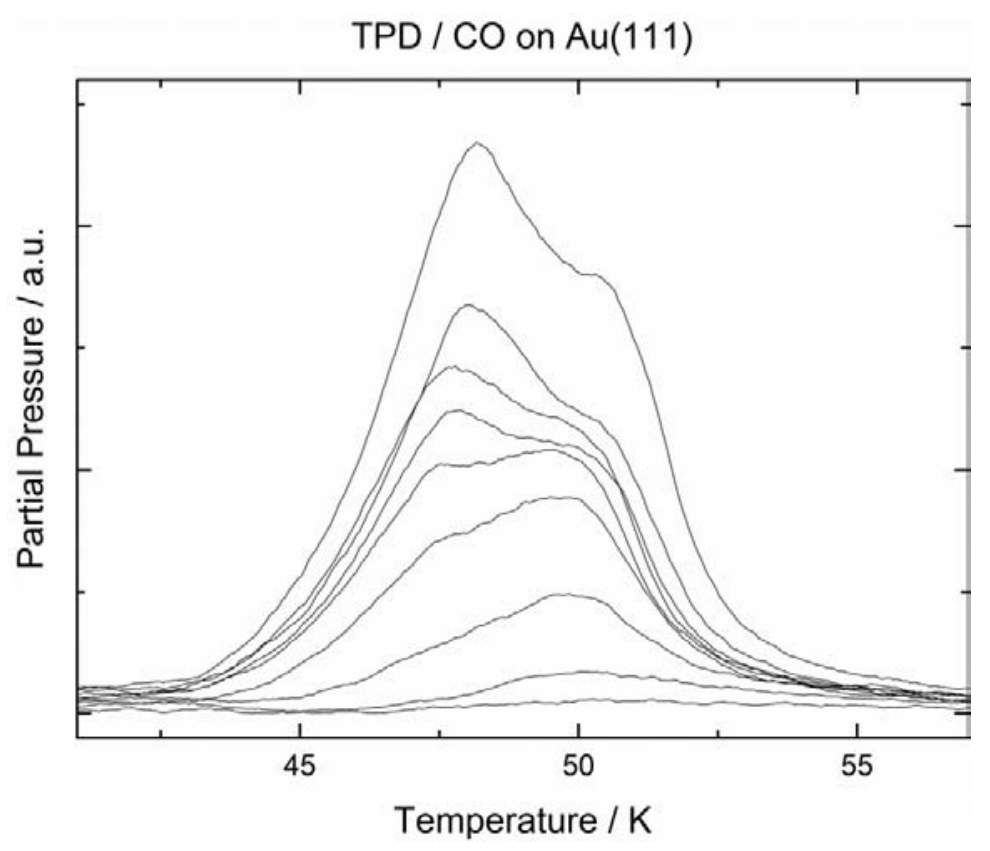

Figure 34 TPD series for sub-monolayer coverages of $\mathrm{CO}$ adsorbed on $\mathrm{Au}(111)$ taken with heating rates of $k=0.5 \mathrm{~K} / \mathrm{s}$. The spectra correspond to the following initial coverages (in ML): $1.0,0.78,0.54,0.50,0.46,0.40,0.31,0.17,0.07,0.02$. The monolayer peak of $\mathrm{CO}$ shows a double structure, caused by a phase transition between two phases with different densely packed structures. See text for more information. 
In addition to line shape analysis, TPD spectra have also been analyzed based on the Polanyi-Wigner relation (equation 2.15):

$$
r=-\frac{d \theta}{d t}=v(\theta) \theta^{n} \exp \left(-E_{\mathrm{des}}(\theta) / R T\right)
$$

where $r$ is the rate of desorption, $\theta$ is the adsorbate coverage in units of monolayers (ML), $t$ is time, $v$ is the pre-exponential factor, $n$ is the order of desorption, $E_{\text {des }}$ is the desorption activation energy, $R$ is the gas constant, and $T$ is surface temperature.

By plotting $\ln \left(r / \theta^{n}\right)$ vs. $1 / T$, we obtain the desorption activation energy, $E_{\text {des }}$, and the pre-exponential factor, $v$, as well as confirming, $n$, the order of desorption kinetics. Figure 35 shows a Polanyi-Wigner plot for the $\mathrm{Kr} / \mathrm{Au}(111)$ system, following the approach presented by Niemantsverdriet. ${ }^{74}$ There is no observable dependence of $E_{\mathrm{des}}$ or $v$ on coverage between 0.2 and 1.0 ML. In this type of plot, the leading edge of the monolayer desorption peaks overlap only when the correct order of desorption is assumed. For all analyses, this analysis confirmed the desorption order suggested by line shape analysis. Results are summarized for all adsorbates in Table 3. Due to overlapping peaks, the Niemantsverdriet analysis cannot be applied to the CO monolayer. 


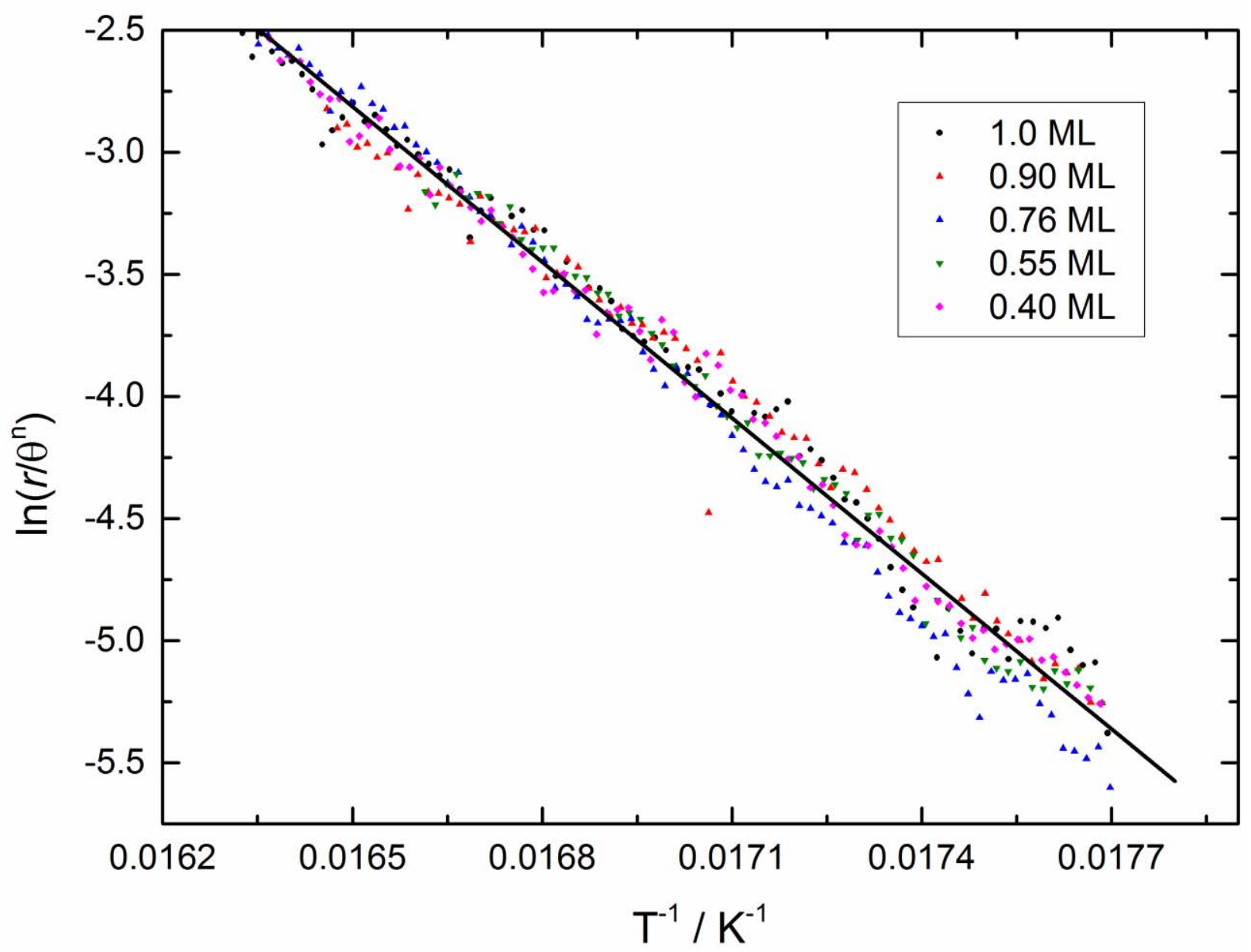

Figure 35 Polanyi-Wigner plots for various Krypton coverages adsorbed on $\mathrm{Au}(111)$. The straight line corresponds to $E_{\mathrm{des}}=0.24 \mathrm{eV}$ and $v=6 \times 10^{14} \mathrm{~s}^{-1}$. We see no dependence of the desorption activation energy on the initial coverage.

In the case of first order desorption kinetics, the desorption process can be analyzed using heating rate variation analysis with the relation:

$$
\ln \left(\frac{T_{\mathrm{M}}^{2}}{k}\right)=\frac{E_{\mathrm{des}}}{\mathrm{R} T_{\mathrm{M}}}+\ln \left(\frac{E_{\mathrm{des}}}{v \mathrm{R}}\right)
$$

where $T_{\mathrm{M}}$ is the surface temperature corresponding to the desorption peak maximum and all other variables are as defined for equation 2.15. To implement this analysis, $T_{M}$ was recorded at different heating rates for initial coverage of $3 \mathrm{ML} \cdot{ }^{101}$ By plotting $\ln \left(\frac{T_{\mathrm{M}}^{2}}{k}\right)$ versus $1 / T_{\mathrm{M}}$, the desorption activation energy can be obtained from the slope of the plot and the pre-exponential factor can be obtained from the $1 / T_{\mathrm{M}}$-intercept. This method was used for $\mathrm{N}_{2}, \mathrm{NO}$, and $\mathrm{CO}-$ all 
adsorbates which exhibit first order desorption kinetics. $E_{\text {des }}$ for $\mathrm{NO}$ and $\mathrm{N}_{2}$ agree well with the values calculated using the Arrhenius-style plots, but the pre-exponential factors differ significantly, indicating large uncertainty in their values. Figure 36 shows the heating rate analysis applied to $\mathrm{N}_{2} / \mathrm{Au}(111)$.

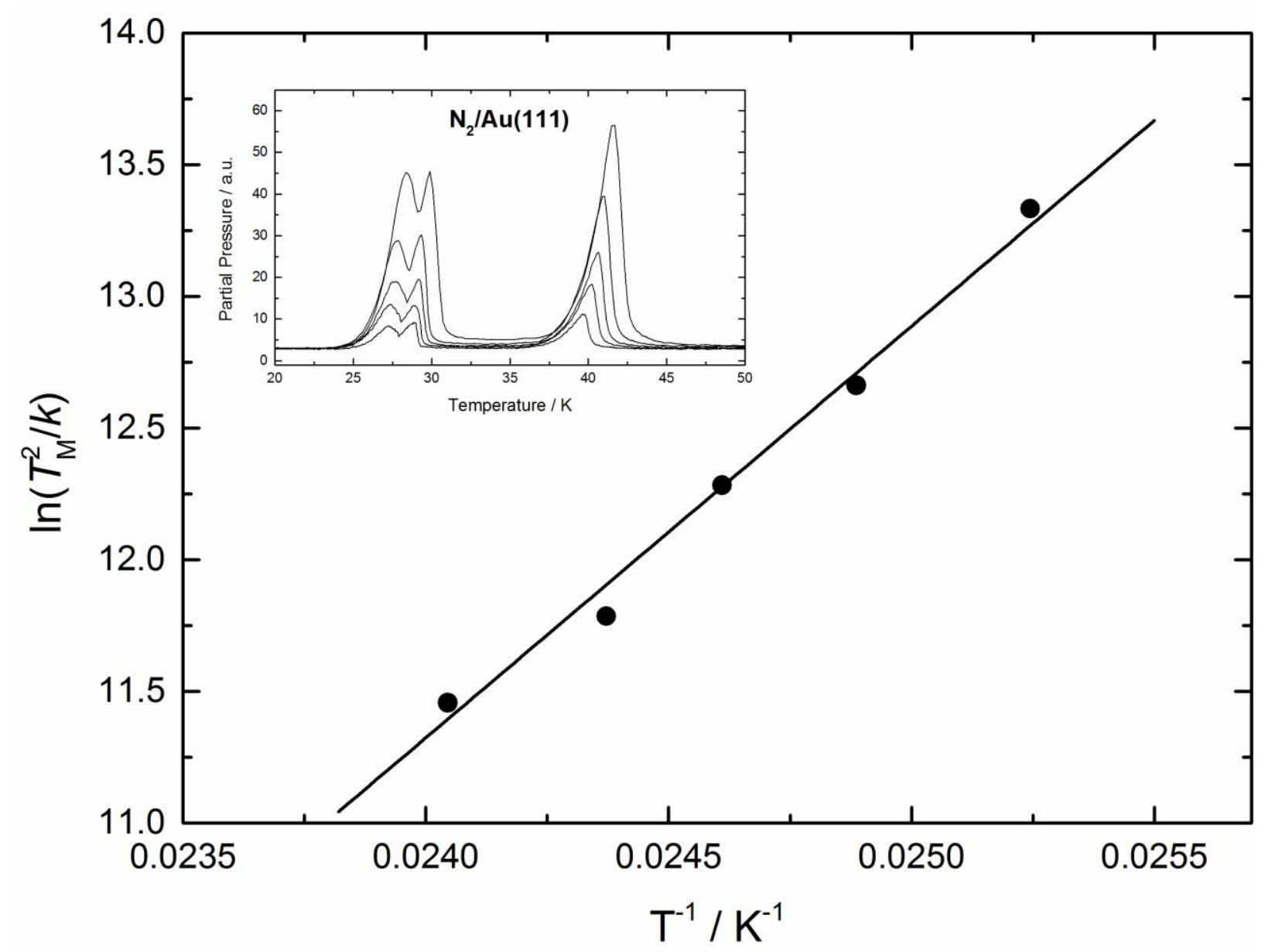

Figure 36 Heating rate variation analysis of $\mathrm{N}_{2} / \mathrm{Au}(111)$ : The slope of the fitted line corresponds to $0.13 \mathrm{eV}$ desorption activation energy; the -intercept gives an Arrhenius preexponential factor of $2 \times 10^{14} \mathrm{~s}^{-1}$. The inset shows TPD spectra with initial coverages of ca. $3 \mathrm{ML}$ recorded at $k=10,20,30,50,70 \mathrm{~K} / \mathrm{min}$, from which the peak maxima are taken. 
Table 3: Desorption activation energies, $E_{\mathrm{des}}$, and Arrhenius pre exponential factors, $v$, for each measured adsorbate's monolayer peak. Values for systems indicated with superscript a) were determined using leading edge plots. Values for systems indicated with superscript b) were determined from a heating rate variation analysis. See text for more details.

\begin{tabular}{|ccc|}
\hline Adsorbate & $\boldsymbol{E}_{\text {des }}$ & $\boldsymbol{v}$ \\
\hline $\mathrm{Ar}^{\mathrm{a})}$ & $0.14 \pm 0.02 \mathrm{eV}$ & $7 \times 10^{14 \pm 2} \mathrm{~Hz}$ \\
$\mathrm{Kr}^{\mathrm{a})}$ & $0.19 \pm 0.02 \mathrm{eV}$ & $6 \times 10^{14 \pm 1.5} \mathrm{~Hz}$ \\
$\mathrm{Xe}^{\mathrm{a})}$ & $0.24 \pm 0.02 \mathrm{eV}$ & $2 \times 10^{12 \pm 1.5} \mathrm{~Hz}$ \\
$\mathrm{C}_{2} \mathrm{H}_{2}{ }^{\mathrm{a})}$ & $0.31 \pm 0.02 \mathrm{eV}$ & $3 \times 10^{15 \pm 1.5} \mathrm{~Hz}$ \\
$\mathrm{SF}_{6}{ }^{\text {a) }}$ & $0.35 \pm 0.03 \mathrm{eV}$ & $3 \times 10^{16 \pm 1.5} \mathrm{~Hz}$ \\
$\mathrm{~N}_{2}{ }^{\mathrm{a})}$ & $0.12 \pm 0.01 \mathrm{eV}$ & $2 \times 10^{15 \pm 1.5} \mathrm{~Hz}$ \\
$\mathrm{NO}^{\text {a) }}$ & $0.23 \pm 0.02 \mathrm{eV}$ & $2 \times 10^{17 \pm 1.5} \mathrm{~Hz}$ \\
\hline $\mathrm{NO}^{\mathrm{b})}$ & $0.24 \pm 0.02 \mathrm{eV}$ & $5 \times 10^{15 \pm 1.5} \mathrm{~Hz}$ \\
$\mathrm{~N}_{2}{ }^{\mathrm{b})}$ & $0.13 \pm 0.02 \mathrm{eV}$ & $2 \times 10^{14 \pm 1.5} \mathrm{~Hz}$ \\
$\mathrm{CO}(\alpha)^{\mathrm{b})}$ & $0.18 \pm 0.02 \mathrm{eV}$ & $2 \times 10^{15 \pm 1.5} \mathrm{~Hz}$ \\
$\mathrm{CO}^{(\beta)^{\mathrm{b})}}$ & $0.15 \pm 0.01 \mathrm{eV}$ & $2 \times 10^{13 \pm 1.5} \mathrm{~Hz}$ \\
\hline
\end{tabular}




\subsubsection{Discussion}

Desorption activation energies and pre-exponential factors have been derived from the TPD measurements reported in this work. In order to relate these kinetic parameters to the binding energy of the adsorbate to the surface, the data were analyzed following the approach developed by Bethune, et al. ${ }^{67}$ In the work of Bethune, the $\mathrm{Xe} / \mathrm{Pt}(111)$ desorption system was analyzed according to the principles of transition state theory (TST). For temperatures below 120 $\mathrm{K}$, the Boltzmann energy distribution is very narrow with respect to the physisorption well depth. At these temperatures, therefore, a particle trapped in this well can be very accurately described by an equilibrium canonical distribution. This is the basis of the TST calculations of desorption rate. The second assumption made by Bethune, et $\mathrm{al}^{67}$ is that at temperatures below $200 \mathrm{~K}$ the sticking coefficient can be approximated by 1 . This is important as the TST expression for desorption depends on a dynamical correction factor which is calculated by simulating the inverse of trapping. The third assumption made is that the surface atoms remain at their equilibrium positions. Briefly, the rate of desorption is calculated by the classical TST expression:

$$
k_{\mathrm{TST}} \equiv v_{\mathrm{t}} \exp \left(-\Delta U_{\mathrm{t}} / k T\right)
$$

where $k_{\mathrm{TST}}$ is the calculated desorption rate, $v_{\mathrm{t}}$ is the calculated temperature dependent preexponential factor, $-\Delta U_{\mathrm{t}}$ is the maximum adsorption well depth, $k$ is the Boltzmann constant, and $T$ is surface temperature. Using this expression, a plot of $\log \left(k_{T S T}\right)$ vs. $1 / T$ is compared to an analogous plot of the experimental data and $-\Delta U_{\mathrm{t}}$ is adjusted to provide the best fit.

This analysis showed the depth of the physisorption well of $\mathrm{Xe} / \mathrm{Pt}(111)$ to be approximately $10 \mathrm{meV}$ deeper than the experimentally measured $E_{\mathrm{des}}$. This difference arises due to the temperature dependence of the pre-exponential factor. This analysis was applied to the $\mathrm{Xe} / \mathrm{Au}(111)$ system, which yielded the same $10 \mathrm{mV}$ discrepancy. As this discrepancy is within the uncertainty of our measurements, this analysis suggests that the experimentally determined values of $E_{\text {des }}$ can be used as approximate binding energies for systems for which desorption occurs at such low temperatures.

Figure 37 shows a plot of each adsorbate's measured $E_{\text {des }}$ in relation to its gas phase

polarizability. ${ }^{102}$ Among the molecular adsorbates, the highest $E_{\mathrm{des}}$ has been found for $\mathrm{SF}_{6}$, 
which also exhibits the highest polarizability. Clearly, for most species studied in this work, $E_{\text {des }}$ increases with increasing polarizability, suggesting pure physisorption. $E_{\text {des }}$ of $\mathrm{NO}$ and $\mathrm{C}_{2} \mathrm{H}_{2}$ do not fall directly on the trend line set by the other adsorbates. Both molecules are known to interact strongly with metal surfaces due to their electronic structures. ${ }^{102-105}$ Acetylene can bond with metal surfaces via $\pi$ electron donation and $\pi^{*}$ back-bonding from the surface and the halffilled electronic orbital in the NO radical can donate or accept electron density to or from the surface. Hence, it is not unreasonable to expect that $E_{\text {des }}$ of these two molecules on $\mathrm{Au}(111)$ is influenced by some degree of charge transfer.

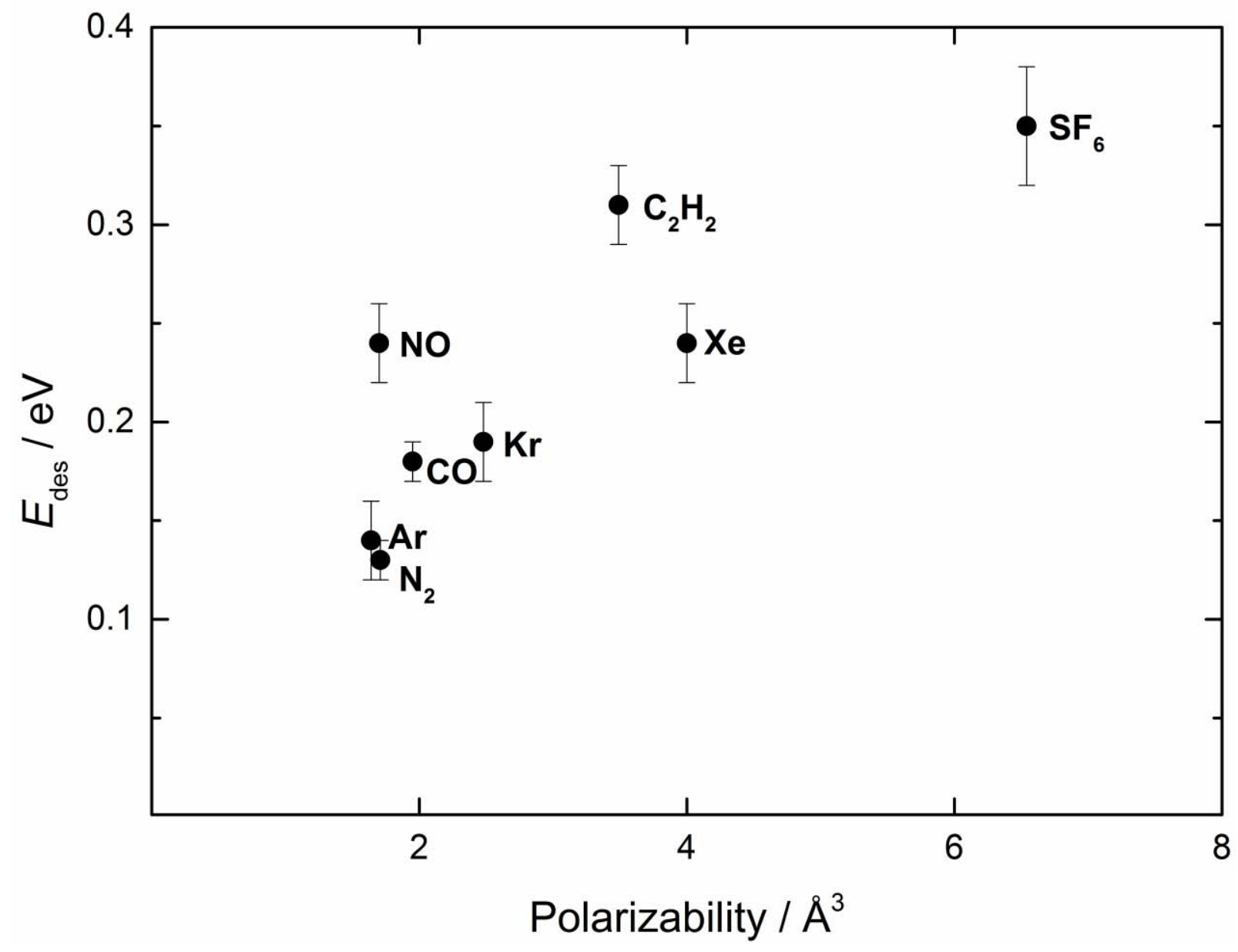

Figure 37 Plot of each adsorbate's electric dipole polarizability vs. derived desorption activation energy. $E_{\text {des }}$ scales with polarizability, indicating physisorption. The higher than expected desorption activation energies of $\mathrm{NO}$ and $\mathrm{C}_{2} \mathrm{H}_{2}$ suggest that their bonds with the surface are not purely due to dispersion forces. See text for more details. 
Although the pre-exponential factor, $v$, is attended by a large uncertainty, it provides trends in the desorption kinetics using the principles of transition state theory. ${ }^{106}$ The preexponential factor is directly connected to the entropy change during desorption, which allows predictions concerning the mobility of the bound state. The change in entropy from a mobile bound state to the transition state is considerably smaller than the entropy change from an immobile state, due to the mobile state's additional degrees of freedom parallel to the surface. Hence, the pre-exponential factor for desorbing mobile adsorbates is usually in the range of $10^{12-13} \mathrm{~s}^{-1}$, whereas the pre-exponential factor for immobile adsorbates can be on the order of $10^{15} \mathrm{~Hz} \cdot{ }^{106}$ Most pre-exponential factors presented in this study indicate immobile adsorption states.

\subsubsection{Conclusion}

We performed TPD experiments using $\mathrm{Ar}, \mathrm{Kr}, \mathrm{Xe}, \mathrm{C}_{2} \mathrm{H}_{2}, \mathrm{SF}_{6}, \mathrm{~N}_{2}, \mathrm{NO}$ and $\mathrm{CO}$ desorbing from a $\mathrm{Au}(111)$ substrate and deduced desorption activation energies of each gas/surface system. These activation energies are an excellent proxy for the experimental binding energies. They scale with the polarizability of the molecule indicating the stability is dominated by dispersion forces. This data set provides a bench mark for theories of dispersion forces for adsorbates at metal surfaces. These results will further serve to define important experimental parameters for surface scattering experiments on adsorbate covered surfaces as will be described in the next section. 


\subsection{Electron emission from clean and adsorbate covered $\mathrm{Au}(111)$ surface}

During gas phase collisions, a highly excited metastable atom can ionize an atom with an ionization potential $E_{\mathrm{IP}}$ lower than the metastable atom's excitation energy, $E^{*} \cdot{ }^{107}$ This results in the release of an electron by the ionized atom and is known as Penning ionization. Similarly, an electronically excited particle can relax to its electronic ground state near a metal surface and the energy lost by the particle can promote an electron into vacuum if the metastable's internal excitation energy exceeds the work function of the metal. An analysis of the electrons emitted due to metastable atom quenching at surfaces proves to be extremely sensitive to density of electronic states near the surface. ${ }^{8}$ This surface sensitivity is used in the related electron spectroscopies metastable quenching spectroscopy (MQS) and ion neutralization spectroscopy (INS). ${ }^{108}$ The results and discussion presented in this chapter characterize the quenching process of metastable $\mathrm{CO}^{*}$ molecules at a clean $\mathrm{Au}(111)$ surface and at the rare gas (RG) adsorbate covered systems $\mathrm{RG} / \mathrm{Au}(111)$ where $\mathrm{RG}$ is $\mathrm{Ar}, \mathrm{Kr}$, or Xe. The TPD data described in the previous chapter served as important input for these experiments by characterizing the temperature dependence of adsorbate coverage.

The purpose of this work is to elucidate the mechanism by which $\mathrm{CO}^{*}$ quenches at the $\mathrm{Au}(111)$ surface and electrons are emitted. The study consists of three parts. First, the determination of the absolute electron emission probability led to the discovery of an enhanced electron emission probability due to vibrational excitation of the impinging molecule. The second part of the study characterizes this vibrational enhancement. The third part characterizes the effect of rare gas adsorbates on electron emission probability. The results of these studies show clear evidence that $\mathrm{CO}^{*}$ quenches at the $\mathrm{Au}(111)$ surface via formation of a transient anionic shape resonance and electrons are emitted due to subsequent electron auto-detachment from the molecule.

\subsubsection{Possible mechanisms of de-excitation}

Electron emission from long lived electronically excited (metastable) rare gas atoms quenching at surfaces has been studied extensively in recent years. A beam of electronically excited atoms can be prepared with well-defined excitation energy using electron bombardment or discharges from cold or hot cathodes, ${ }^{108}$ then scattered from a surface of interest. When the molecule relaxes to the ground state at the surface, most of its excitation energy is used to excite 
electrons in the surface, which can lead to electron emission into vacuum (exoelectrons). The resulting kinetic energy spectrum of the ejected electrons contains information on the electronic state of the surface. In contrast to exoelectron spectroscopies which use electrons or photons as probes, the incident metastables do not penetrate into the bulk, so the topmost atomic layers of the surface can be selectively probed.

De-excitation of electronically excited atoms at metal surfaces proceeds by one of two mechanisms, depending on the excitation energy of the incident atom and the work function of the metal. ${ }^{8,17,18,108}$ The left panel of Figure 38 shows the Auger de-excitation mechanism. If the ionization energy $\left(E_{\mathrm{IP}}\right)$ of the incident particle is larger than the work function of the solid, $\Phi$, an electron in the solid transfers to the vacant low-lying orbital in the atom and the electron in the atom's excited orbital can be ejected.

If the $E_{\mathrm{IP}}$ of the electronically excited atom is smaller than the work function of the metal surface, the excited electron in the molecule can transfer to an unoccupied orbital in the metal's conduction band, shown in the right panel of Figure 38. This process is known as resonant ionization (RI). After electron transfer from the atom to the metal, the atom is neutralized via an Auger neutralization (AN) process in which one electron from the solid fills a low lying unoccupied orbital of the atom and another electron is ejected from the metal into vacuum. 


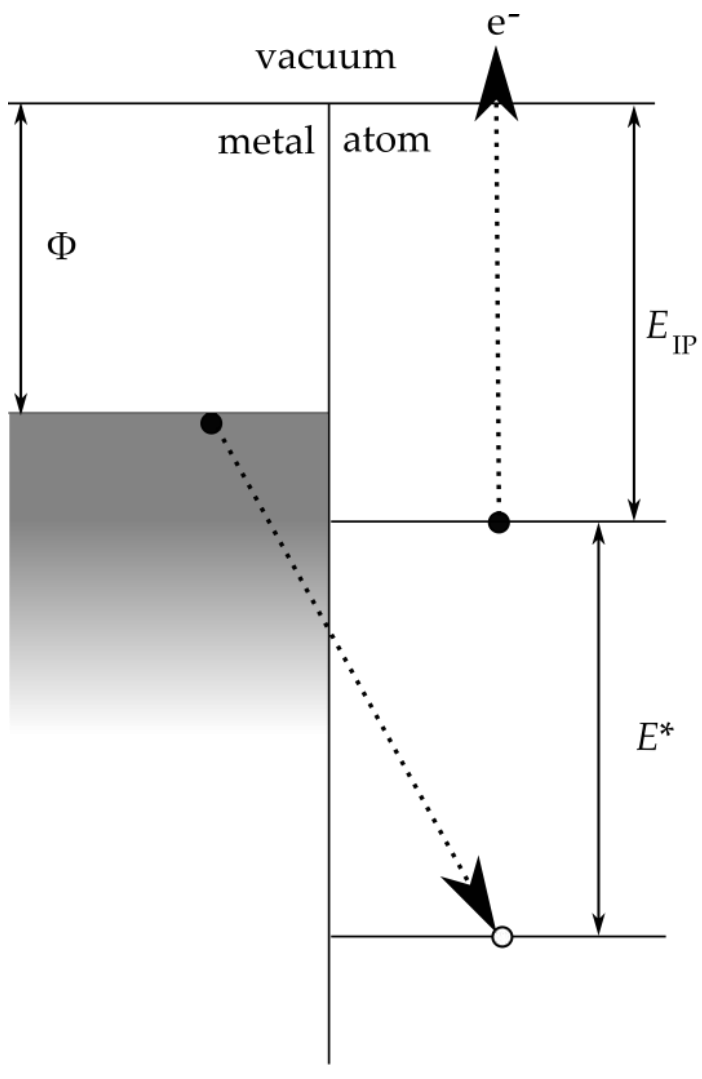

AD

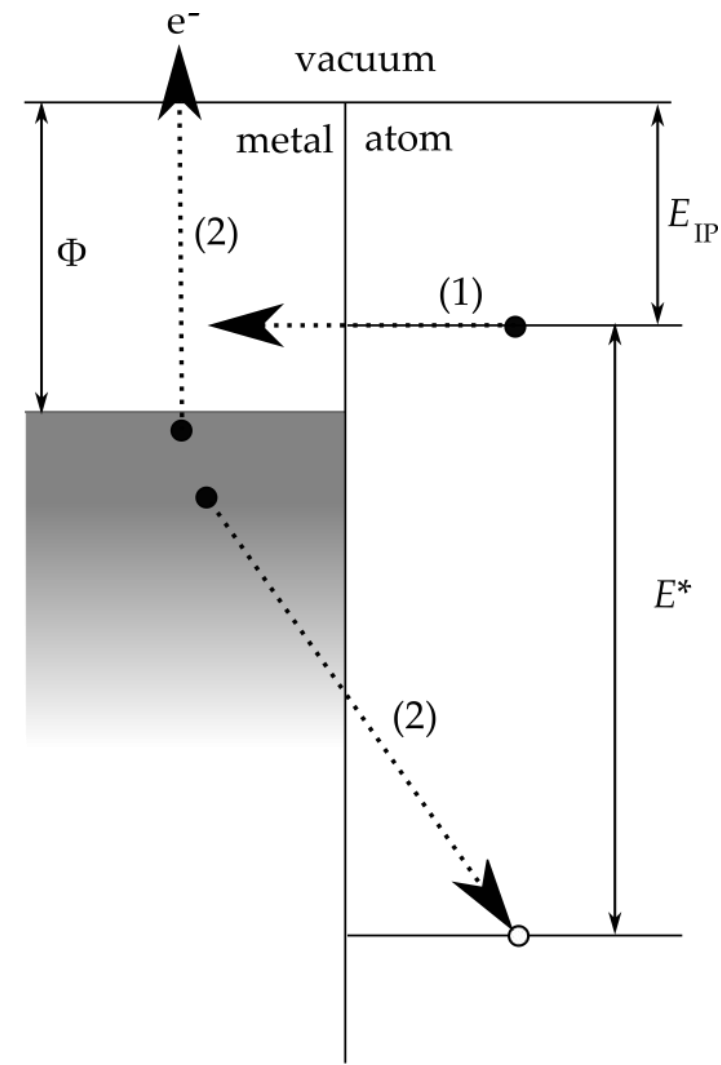

RI+AN

Figure 38 Left panel: Auger de-excitation (AD) of an electronically excited particle at a metal surface. An electron from near the Fermi level of the metal relaxes to the unfilled orbital in the electronically excited $\mathrm{CO}$, simultaneously ejecting the particle's excited electron into vacuum. Right panel: in the first step, the electron in the excited orbital of the metastable tunnels to an unoccupied energy level in the metal (1). Subsequently, an electron from the surface fills the low-lying electronic orbital in the ion, simultaneously ejecting an electron from the metal into vacuum. See text for more discussion.

Clearly, if $E_{\mathrm{IP}}$ is smaller than $\Phi$, then electronic de-excitation can proceed by either AD or RI+AN. In the case that both mechanisms are allowed, RI+AN dominates the de-excitation process. ${ }^{108}$ This phenomenon arises because AD is only expected to be efficient at surface distances between 3-5 $\AA$, whereas RI+AN occurs with reasonable probabilities at surface-particle distances greater than $5 \AA^{10,12,108}$ Electron emission due to either process is possible if $E^{*}>\Phi$. Of the two mechanisms discussed thus far, only $A D$ mechanism is reasonable for the $\mathrm{CO}^{*} / \mathrm{Au}(111)$ system as the ionization energy of $\mathrm{CO}^{*}(8.0 \mathrm{eV})$ is greater than the work function of $\mathrm{Au}(111)(5.3 \mathrm{eV})$. The $\mathrm{AD}$ and $\mathrm{RI}+\mathrm{AN}$ models successfully describe the electron emission 
induced by metastable atoms at surfaces. For both processes, electron emission probability, $\gamma$, increases with increasing excess energy, $E_{\mathrm{ex}}$, where

$$
E_{\mathrm{ex}}=E^{*}-\Phi
$$

and is found to be nearly independent of the atom's translational energy in the low energy regime $(<50 \mathrm{eV}) .{ }^{108}$

The energetics of molecules are considerably more complex than that of atoms, however. Because molecules consist of at least two atoms, they can store energy in degrees of freedom which are not available to atoms. For example, the effect of vibrational excitation on the electron emission process is not addressed by these models. The model also neglects any effect of orientation or rotational excitation. Earlier work by White and coworkers ${ }^{24-27}$ has shown that vibrational degrees of freedom of a scattering molecule can couple very strongly with electrons at a metal surface, sometimes leading to electron emission. This stronger-than-expected coupling of the molecule's vibrational energy to the surface electrons is mediated by electron transfer. Later work by Bartels et al. has shown that the energetic coupling of rotationally and vibrationally excited molecules with surface depends crucially on the scattered molecule's orientation. ${ }^{92,96}$ Therefore, it is not reasonable to expect that quenching of molecular metastables at surfaces can be completely described by theories developed to explain metastable atomsurface scattering.

Molecules at surfaces can undergo a variety of processes that can lead to chemical reactions and surface modifications. Nearly all molecules, for example, can bind an electron and form a negative ion. For example, when a slow neutral molecule approaches a metal surface an electron can transfer from the surface to the molecule, forming a negative ion at moleculesurface distances larger than those typical for chemisorption, i.e. $\geq 1.5 \AA{ }^{109,} 110$ Therefore, electron transfer from the surface to incoming metastable molecule may be an important step in quenching, as has been suggested in several publications. ${ }^{111-115}$ Subsequent decay of the anion to the neutral molecule can lead to emission of electrons into vacuum. See Figure 39. This process has been invoked to explain the neutralization and subsequent electron emission of metastable $\mathrm{N}_{2}$ quenching at an aluminum surface. Hereafter, this mechanism shall be referred to as anion mediated (AM) de-excitation. 


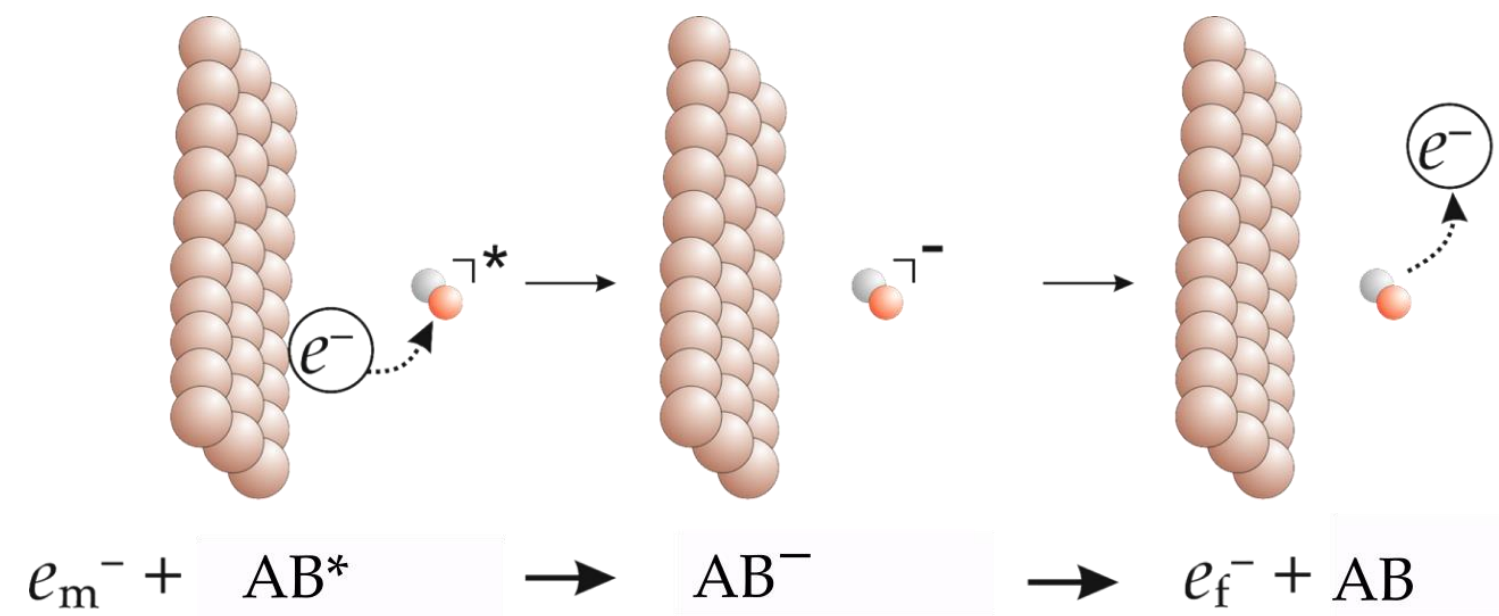

Figure 39 De-excitation of metastable molecule $\mathrm{AB}^{*}$ via an anion mediated mechanism. In the first step the molecule captures an electron from the metal surface. The anion subsequently decays to the electronic ground state of the neutral molecule and emits an electron. ${ }^{116}$

It should be noted that the formation of an anion changes the interaction of the molecule with the surface due to Coulomb interaction. When a molecule approaches a metal surface its electron affinity increases due to image interaction with the surface. ${ }^{18}$ The energy of any ionic state is reduced from its gas phase value as it approaches a metal surface due to interaction between the ion and its image in the solid. The image charge stabilization is calculated according to the formula:

$$
\Delta E_{\text {ion }}=-\frac{3.6}{(z-d)} \mathrm{eV} \AA
$$

where $z$ is the distance from the center of the particle to the jellium edge of the metal in which the positive ions of the metal are represented by a uniform background and $d$ is a correction factor usually estimated as 0.5-0.6 $\AA^{108,117,118}$

At some finite molecule-surface distance, the molecule's electron affinity will exceed the surface work function and an electron can tunnel from the surface to the molecule, forming a negative ion, see Figure 40. Note that in this figure the vacuum level of the metal surface is set equal to the occupied metastable electronic orbital of the incoming molecule in order to compare electron affinities. 


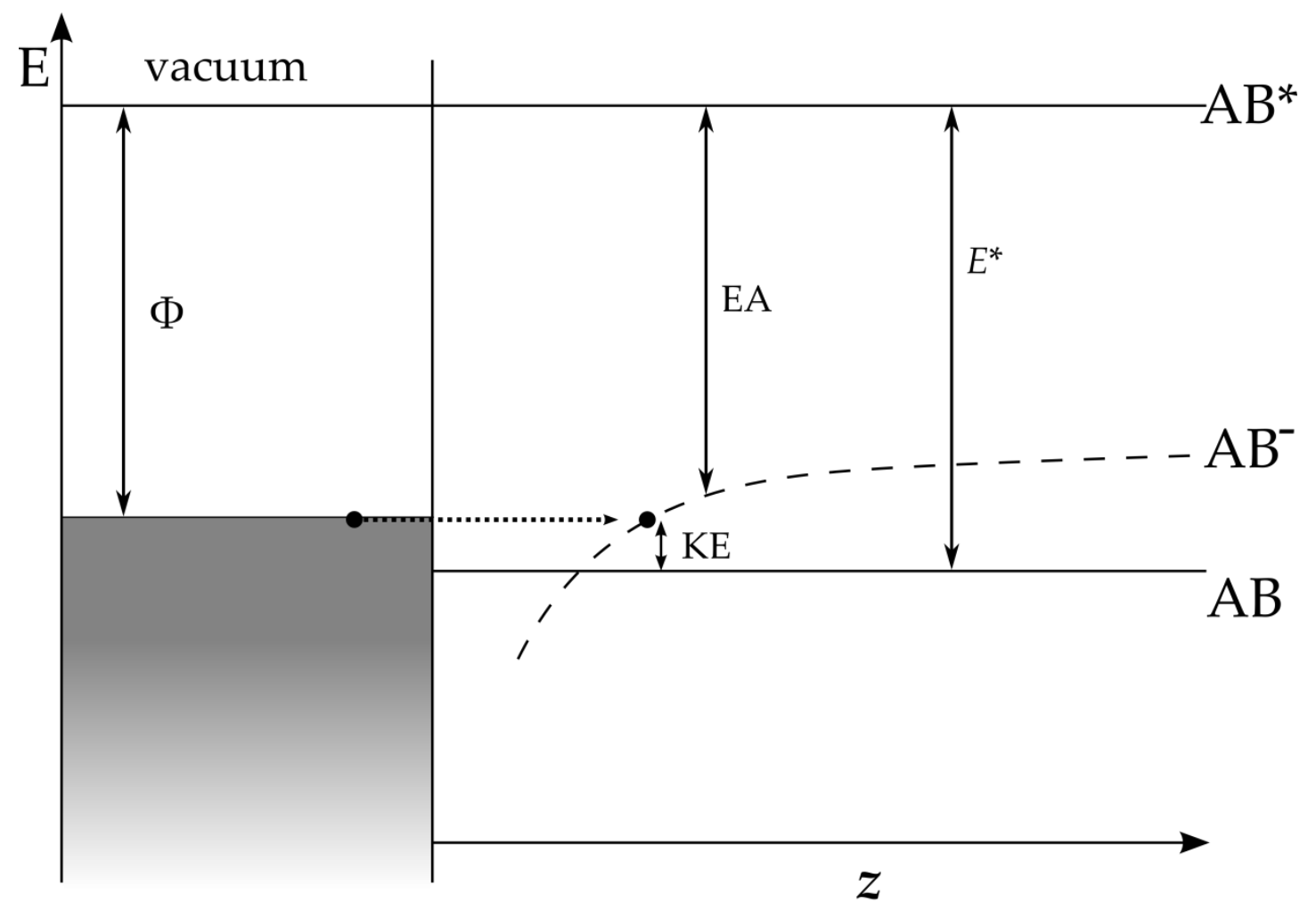

Figure 40 Schematic picture of the de-excitation of a metastable molecule at a metal surface. The left side represents the metal surface, $\Phi$ is the work function of the metal. The metastable molecule approaches the surface with $E^{*}>\Phi$. As the molecule approaches the surface, the lowlying unoccupied anionic resonance (dashed line) is stabilized due to image charge interaction. The molecule's electron affinity (EA) increases with decreasing surface distance until the molecule can capture a metal electron. The resulting anion then auto-detaches an electron with a kinetic energy (KE) which depends on the energy difference between the anion and the ground electronic state of the neutral, as indicated.

Both ionization of the metastable molecule by a surface electron and the decay of the anion to the neutral state are one electron processes. Thus, they can be expected to be more efficient than a simultaneous two electron Auger process. The anion mediated process should also be efficient in the case where direct de-excitation of the parent molecule is spin forbidden, as an electron from the surface in one spin state can be captured and another electron in a different spin state can be ejected. 
In the following sections, the electron emission resulting from $\mathrm{CO}^{*}$ quenching on $\mathrm{Au}(111)$ will be quantified and analyzed within the framework of the AD and AM models. It will be shown that the higher than expected value of $\gamma$ resulting from the quenching process, the enhancement of $\gamma$ with vibrational excitation of the incident molecule, and the dependence of $\gamma$ on surface-molecule distance and surface adsorbate coverage can all be explained within the framework of an AM mechanism. 


\subsubsection{Absolute Electron Emission Probability}

One clearly defined experimental observable during the de-excitiation process is the production of exoelectrons. The probability of electron emission during the quenching process provides insight into the mechanism of de-excitation. Therefore, the first experiments performed on the newly built apparatus were to measure the absolute emission probability of CO* quenching on atomically clean $\mathrm{Au}(111)$. From a practical perspective, this measurement is crucial as an experimental benchmark with which to characterize the performance of the apparatus.

The measured electron emission probability is $\gamma_{\mathrm{CO}}=0.13$. This value was found to increase with vibrational excitation of the molecule. We developed a simple model to distinguish between the roles played by vibrational and electronic excitation of the molecule in the ejection of electrons into vacuum. Our observations are consistent with an AM de-excitation mechanism while an analysis of electron emission using an AD mechanism modified to take into account vibrational excitation was unable to reproduce our experimental results.

\subsubsection{Experimental and results}

As described in section 2, the $\mathrm{Au}(111)$ surface was prepared by standard cycles of $\mathrm{Ne}^{+}$ sputtering and annealing. Surface purity was confirmed with Auger electron spectroscopy. During all measurements, the target crystal temperature was maintained at $100{ }^{\circ} \mathrm{C}$ in order to minimize surface contamination by residual gases. Carrier gas-free pulses of CO* are created by first expanding a mixture of $20 \% \mathrm{CO}$ in Xe from a cooled pulsed valve. After skimming, the ground state molecules are electronically excited via the $\mathrm{a}^{3} \Pi_{1},(v=0, J=1) \leftarrow \mathrm{X}^{1} \Sigma^{+},(v=$ $0, J=1)$ transition at $206 \mathrm{~nm}$ to create metastable $\mathrm{CO}^{*}$. The $\mathrm{CO}^{*}$ molecules are then separated from the carrier gas and remaining ground state molecules in an electrostatic hexapole filter and the velocity distribution of the resulting pulse is chosen using the Stark decelerator. All measurements reported in the following section have been taken using a $360 \mathrm{~m} / \mathrm{s}$ molecular beam pulse. Molecules travelling toward the surface can be ionized $23 \mathrm{~mm}$ upstream from the surface via a (1+1) REMPI process utilizing the $\mathrm{b}^{3} \Sigma^{+}(v=0) \leftarrow \mathrm{a}^{3} \Pi_{1}(v=0)$ transition at $283 \mathrm{~nm} .{ }^{47}$ $283 \mathrm{~nm}$ laser light was produced using a Nd:YAG pumped Spectra Physics PDL-2 dye laser and frequency doubling in a KDP crystal. This laser is hereafter referred to as the REMPI laser. 
The experiment makes use of three signals: the exoelectron signal arising from a $\mathrm{CO}^{*}$ pulse impinging on the surface, the exoelectron signal arising from a pulse which has been partially ionized before hitting the surface, and the ion signal resulting from the partial ionization of the incident molecular beam pulse. Figure 41 shows a schematic representation of the experimental setup and the origin of the three signals.

The partially ionized beam pulse has a hole burned through it and this hole is clearly visible in time resolved $\mathrm{CO} / \mathrm{Au}(111)$ electron emission signals. By comparing the electron signal arising from non-ionized and partially ionized $\mathrm{CO}^{*}$ pulses, we can determine the effect that the hole-burning has on the electron emission signal. The absolute electron emission yield, $\gamma$, can be calculated by subtracting one electron signal from the other and dividing the corresponding time-integrated time-of-flight spectrum by the integrated ion signal. Prior to these experiments, great care was taken to correct for different MCP gains by normalization to identical signals. 


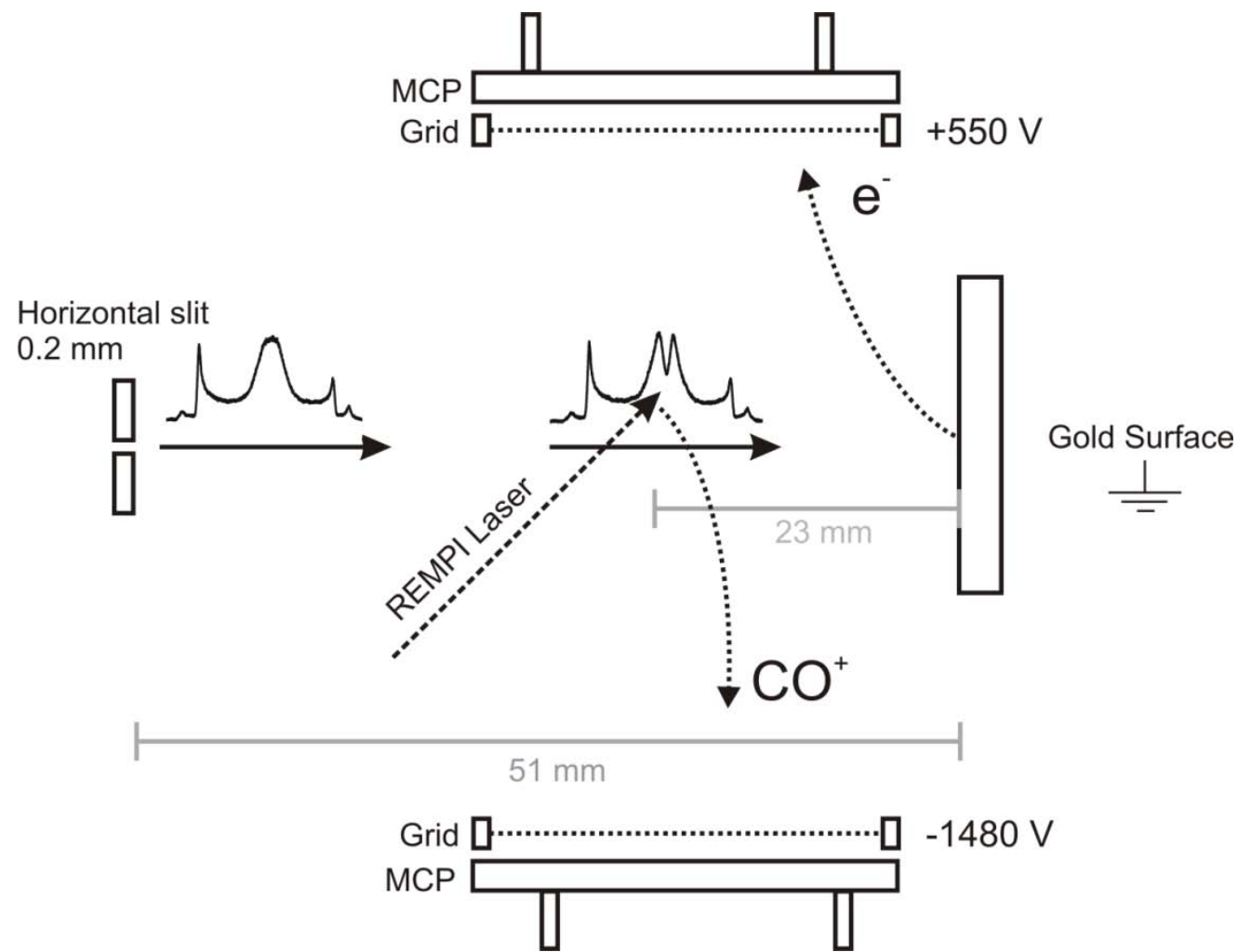

Figure 41 Schematic of experimental setup in front of $\mathrm{Au}(111)$ surface between upper and lower MCP detectors. The molecular beam passes through a horizontal $200 \mu \mathrm{m}$ slit $5.1 \mathrm{~cm}$ upstream from the target surface. The $283 \mathrm{~nm}$ ionization laser is crossed with the CO* pulse 23 $\mathrm{mm}$ before the surface and ionized $\mathrm{CO}$ molecules are accelerated toward the MCP detector mounted below the ionization zone. Subsequently, electrons which are emitted due to nonionized $\mathrm{CO}^{*}$ molecules quenching on the gold surface are detected on a second MCP detector mounted above the surface. Any electrons originating from the REMPI ionization are well separated in time from those emitted near the surface. ${ }^{83}$

The electron signals are shown in Figure 42. Interestingly, "wings" arise on each side of the depletion signal, indicating enhancement of electron emission. Laser intensity is lower on the spatial edges of the laser focus, suggesting that the signal increase is due to molecules which are excited to the short-lived $\mathrm{b}^{3} \Sigma^{+}(v=0)$ state $(\tau=53 \mathrm{~ns})^{119}$ with one $283 \mathrm{~nm}$ photon, but are not ionized and subsequently decay back into different vibrational levels of the $\mathrm{a}^{3} \Pi$ state. This hypothesis was confirmed by observation of electron emission enhancement upon reducing the power of the REMPI laser to approximately $20 \mu \mathrm{J} /$ pulse. This laser power is insufficient to ionize molecules via the 1+1 REMPI transition, but resulted in significant enhancement of the electron signal over the unperturbed $\mathrm{CO}^{*}$ beam pulse. 


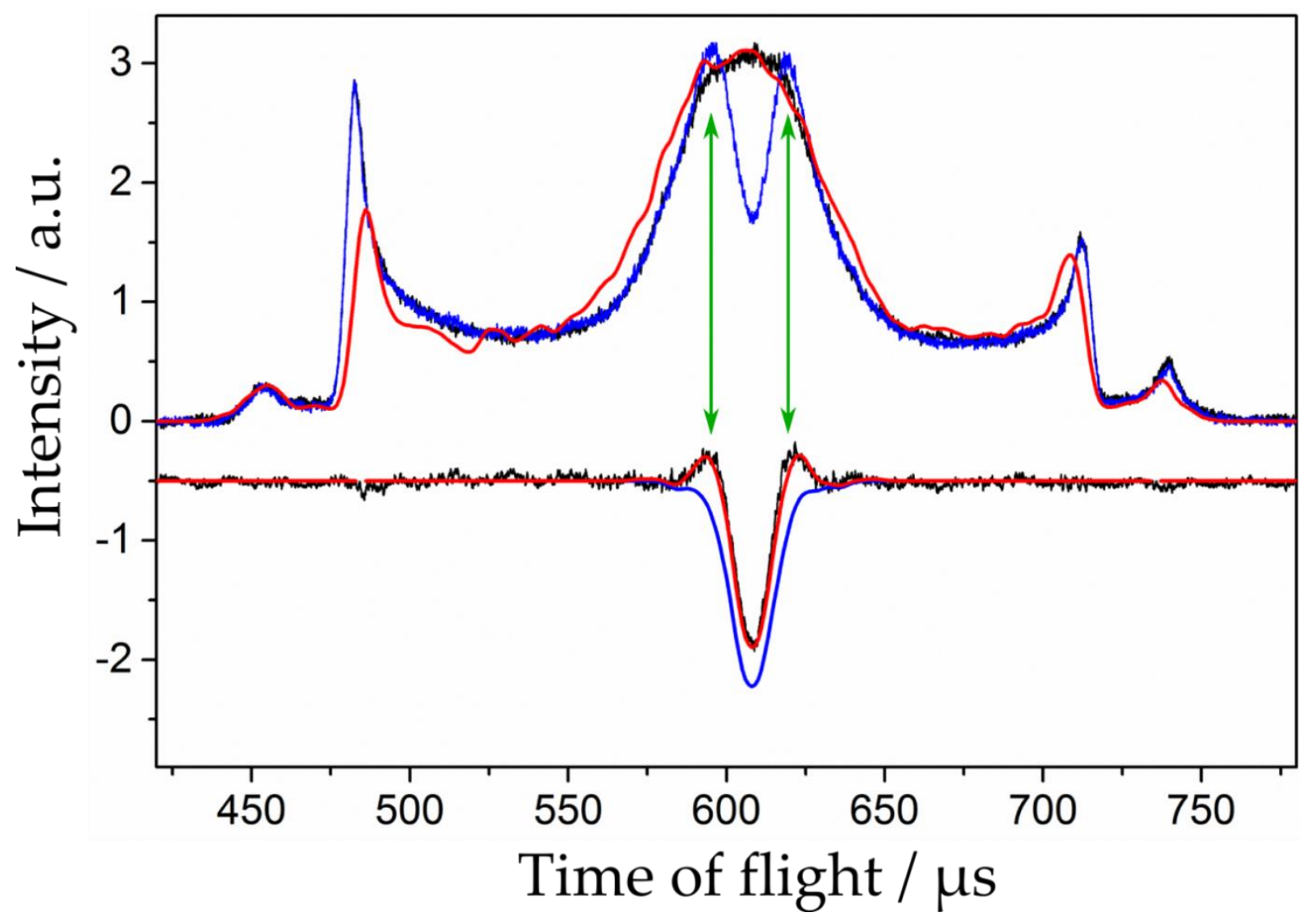

Figure 42 Time resolved signal of electrons emitted during the scattering of $\mathrm{CO}^{*}$ from $\mathrm{Au}(111)$. The upper curves show experimental data (black) and simulated signals (red) of electron emission resulting from the undisturbed pulse and electron emission signal resulting from the depleted pulse (blue). Enhanced wings can be clearly seen in the depleted (upper blue) trace and are indicated with green arrows. The lower curves show the result of the undepleted electron signal subtracted from the depleted signal (black), and fitted with a simple model (red, see text). The lower blue curve depicts the contribution of depletion. The difference between the lower blue and lower red curves is due to vibrational enhancement of electron emission. ${ }^{83}$ The shape of the time-of-flight profile of the electron signals is due to the trajectories of molecules in the Stark decelerator. $^{120}$

Franck-Condon factors of the $\mathrm{b}^{3} \Sigma^{+}\left(v=v^{\prime}\right) \rightarrow \mathrm{a}^{3} \Pi\left(v=v^{\prime \prime}\right)$ transition predict that $75 \%$ of all molecules will fall to $v^{\prime \prime} \geq 1$ after excitation to the $\mathrm{b}$ state. This is a clear indication of vibrational enhancement of electron emission in quenching of $\mathrm{CO}^{*}$. Further evidence was found by scanning the REMPI laser wavelength and monitoring electron emission signal from the surface and the ion signal. During scans, the REMPI laser was not focused in order to minimize ionization and optimize production of vibrationally excited $\mathrm{CO}^{*}$ molecules by optical pumping 
to the $\mathrm{b}^{3} \Sigma^{+}(v=0)$ state. The spectra shown in Figure 43 clearly indicate two distinct peaks assigned to the $R_{32}(1)$ and the overlapping $P_{32}(1)$ and $R_{12}(1)$ transitions. Electron emission enhancement occurs only when the laser is resonant with a REMPI transition. These transitions correspond to production of vibrationally excited $\mathrm{CO}^{*}$. For comparison, the electron signal is also shown in Figure 43. Enhancement of exoelectrons signal coincides with the production of ions, thus proving vibrational enhancement.

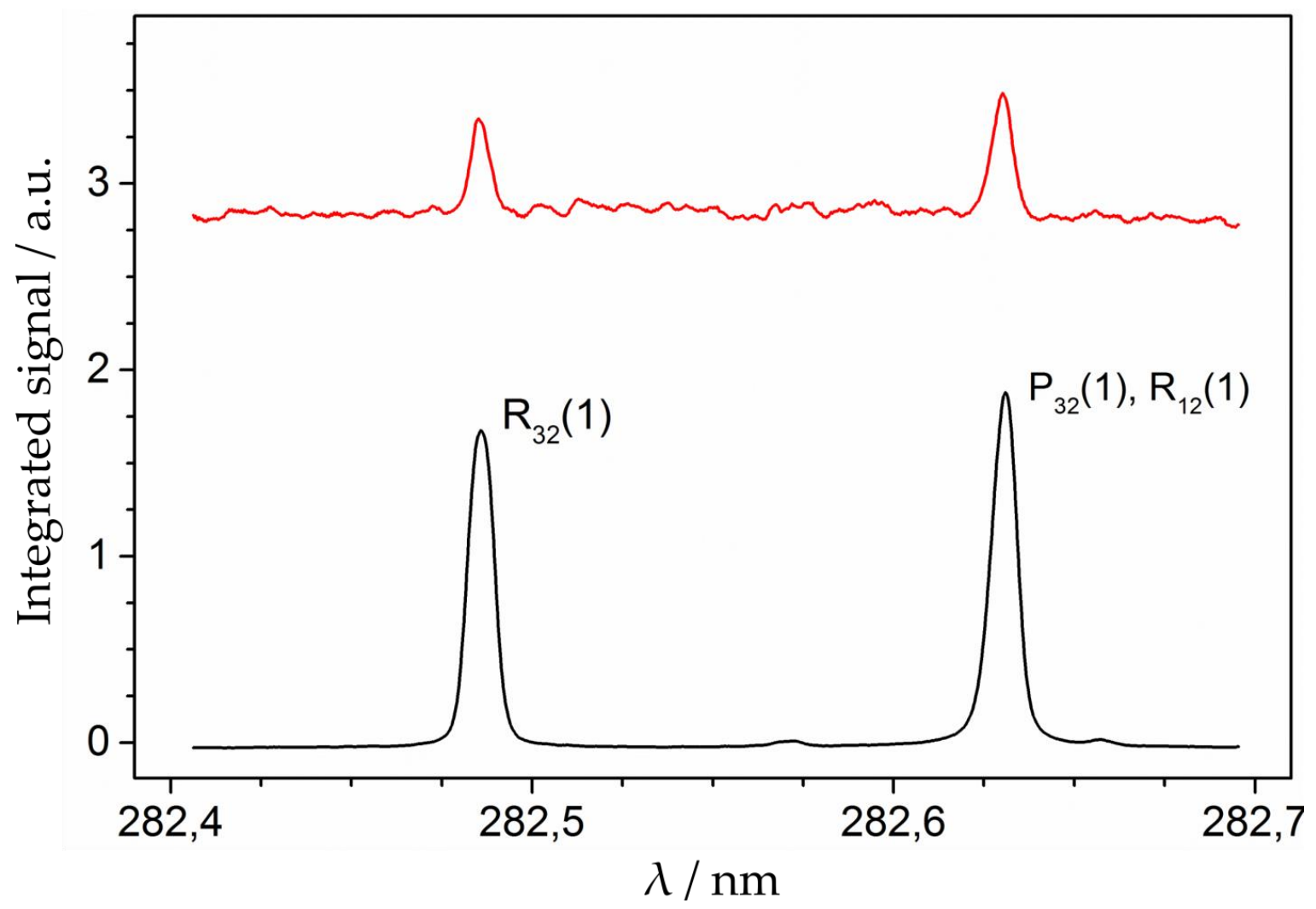

Figure 43 Integrated ion (lower, black) and electron (upper, red) signal recorded as REMPI laser is scanned over the $\mathrm{b}^{3} \Sigma^{+}(v=0) \leftarrow \mathrm{a}^{3} \Pi_{1}(v=0)$ transition. The REMPI laser is unfocused ( $5 \mathrm{~mm}$ diameter) and low intensity ( $20 \mu \mathrm{J} / \mathrm{pulse})$ in order to minimize ionization and increase probability of each molecule interacting with a single photon to produce vibrationally excited $\mathrm{CO}^{*}$ molecules. Electron emission probability is enhanced when the laser is resonant with the $\mathrm{R}_{32}(1)$ and the overlapping $\mathrm{P}_{32}(1)$ and $\mathrm{R}_{12}(1)$ transitions. ${ }^{83}$ 


\subsubsection{Discussion}

In order to calculate the electron emission probability from $\operatorname{Au}(111) / \mathrm{CO}^{*}(v=0)$ collisions, it was necessary to distinguish the contribution of vibrational ground state $\mathrm{CO}^{*}$ from that of vibrationally excited CO*. To disentangle these two signals, we developed a simple model to fit the contributions of signal depletion and enhancement to our electron signal and describe our experimental observations. ${ }^{48,83}$ Briefly, the model uses trajectory calculations of molecules passing through a Stark decelerator, which provide excellent agreement with experiment as seen in Figure 42. ${ }^{37,48}$

To determine the interaction of the molecular beam with the laser pulse, we simply overlap the modeled beam pulse with a modeled cylindrical laser pulse. The laser intensity, $I$, of the pulse is assumed to have a Gaussian intensity distribution. We assume ionization efficiency $\propto I^{2}$, but saturated ionization within one full width at half maximum (FWHM) of $I$. Outside the FWHM, we assume a ring with inner diameter of $1 \times$ FWHM and outer diameter of $3 \times$ FWHM where molecules are only transferred to higher vibrational states, but not ionized. Franck-Condon pumping occurs in this outer ring. The electron signal resulting from the impact of the vibrationally excited molecules is multiplied by an enhancement factor of $\varepsilon>1$. By implementing these simulations and adjusting only 2 parameters, FWHM and $\varepsilon$, we are able to reproduce our data. The best fit resulted in values of $\mathrm{FWHM}=480 \mu \mathrm{m}$ and $\varepsilon=1.31$. Subtracting the enhancement contribution from the peak shown in Figure 42, time-integrating the adjusted depletion signal and dividing by the calibrated and time-integrated ion signal yields

$$
\gamma_{0}=0.13 \pm 0.05
$$

where $\gamma_{0}$ is the absolute electron emission yield of $\mathrm{CO} \mathrm{a}^{3} \Pi_{1}(v=0)$ quenching on an atomically clean $\mathrm{Au}(111)$ surface. This model is useful for determining the electron emission probability for vibrational ground state $\mathrm{CO}^{*}$ quenching on $\mathrm{Au}(111)$, but a qualitative determination of vibrational enhancement will be presented in the following section. An exhaustive discussion of the model and details of the trajectory simulations can be found in Grätz. ${ }^{48}$

This precise knowledge about the quenching process provides a powerful tool for determining density of $\mathrm{CO}^{*}$ molecules in our molecular beam by monitoring electron emission from the target surface. Monitoring electron signal from the surface can be used for shot-to-shot 
normalization of beam density during other measurements. Additionally, knowledge of $\gamma_{\mathrm{CO}}$ allows for a comparison of the $\mathrm{CO} * / \mathrm{Au}(111)$ system to other metastable/metal systems. I will use this knowledge in section 3.2.4 to describe the influence of adsorbates on the quenching process.

As described above, for systems in which a metastable particle quenches on a metal via $\mathrm{AD}, \gamma \propto E_{\mathrm{ex}}$. Comparison of $\gamma_{\mathrm{CO}}$ to the electron emission probability of well documented AD processes yields insight into the nature of the $\mathrm{CO} * / \mathrm{Au}(111)$ interaction. The excitation energy of CO* is $6.0 \mathrm{eV}$ versus $\Phi_{\mathrm{Au}(111)}$ of $5.31 \mathrm{eV}$, leaving $E_{\mathrm{ex}}$ of $0.69 \mathrm{eV}$. Considering the magnitude of excess energy in this scattering process, an electron ejection probability of 0.13 is surprisingly high. This value is comparable to $\mathrm{Kr}^{3} \mathrm{D}_{3}\left(E^{*}=11.4 \mathrm{eV}\right)$ on polyscystalline gold $(\Phi=5.1 \mathrm{eV})$. For this system $E_{\mathrm{ex}}=6.3 \mathrm{eV}$ and $\gamma=0.16$. The emission probability is markedly higher than that for $\mathrm{Kr}^{3} \mathrm{P}_{2}$ on polycrystalline gold $\left(E^{*}=9.9 \mathrm{eV}, E_{\mathrm{ex}}=4.8 \mathrm{eV}, \gamma=0.06\right) .{ }^{121}$ Although these experiments were performed using a polycrystalline gold target surface that was only cleaned by heating to $87^{\circ} \mathrm{C}$, the nobility of gold and the stability of the $\mathrm{Au}(111)$ surface suggest that these measurements provide a useful comparison to our $\mathrm{CO} * / \mathrm{Au}(111)$ studies.

The results presented in this section provide the first suggestion that electron emission from $\mathrm{CO}^{*}$ quenching on $\mathrm{Au}(111)$ cannot be adequately described using $\mathrm{AD}$ and show clear evidence that vibrational excitation of the incoming molecule leads to enhancement of electron emission into vacuum. In the next section, the role of vibrational excitation on electron emission probability will be explored and a hypothetical mechanism for $\mathrm{CO}^{*}$ de-excitation on $\mathrm{Au}(111)$ will be presented. 


\subsubsection{Vibrational enhancement of electron emission}

The absolute electron emission probability, $\gamma$, during the quenching of $\mathrm{CO} *$ on $\mathrm{Au}(111)$, reported in the previous section, is remarkably high and suggests that the de-excitation of electronically excited molecules at metal surfaces may not be well described by an Auger deexcitation (AD) mechanism. Additionally, the electron emission probability was increased when scattering vibrationally excited molecules from the surface. In order to describe the vibrational enhancement of electron emission more precisely, we employ Franck-Condon pumping (FCP) to populate chosen vibrationally excited levels of the incident $\mathrm{CO}^{*}$ molecules under conditions where ionization/depletion are not important. These results provide evidence of transient negative ion formation during the quenching of electronically excited $\mathrm{CO}$ at the $\mathrm{Au}(111)$ surface.

\subsubsection{Experimental and results}

The experiments reported in this section are similar to those reported in the previous section except in one respect. The incident molecular beam pulse was prepared in controlled vibrational state distributions as shown in Figure 44. The pulse of $\mathrm{CO}^{*}$ molecules is pumped to the $\mathrm{b}^{3} \Sigma^{+}(v=0$ or 1$)$ state using pulsed laser light (Continuum Sunlite Ex OPO) crossed with the molecular beam $19 \mathrm{~mm}$ upstream from the surface, hereafter known as the FCP laser. The lifetimes of the $\mathrm{b}^{3} \Sigma^{+}(v=0$ or 1$)$ states are less than $70 \mathrm{~ns},{ }^{119}$ ensuring that all excited molecules fluoresce to vibrational states in the $a^{3} \Pi$ electronic state in Franck-Condon distributions. Laser power during this step is approximately $50 \mu \mathrm{J}$ in a $5 \mathrm{~mm}$ diameter beam, such that it is weak enough to avoid ionization, but strong enough to saturate the $b^{3} \Sigma^{+} \leftarrow a^{3} \Pi$ transition. 


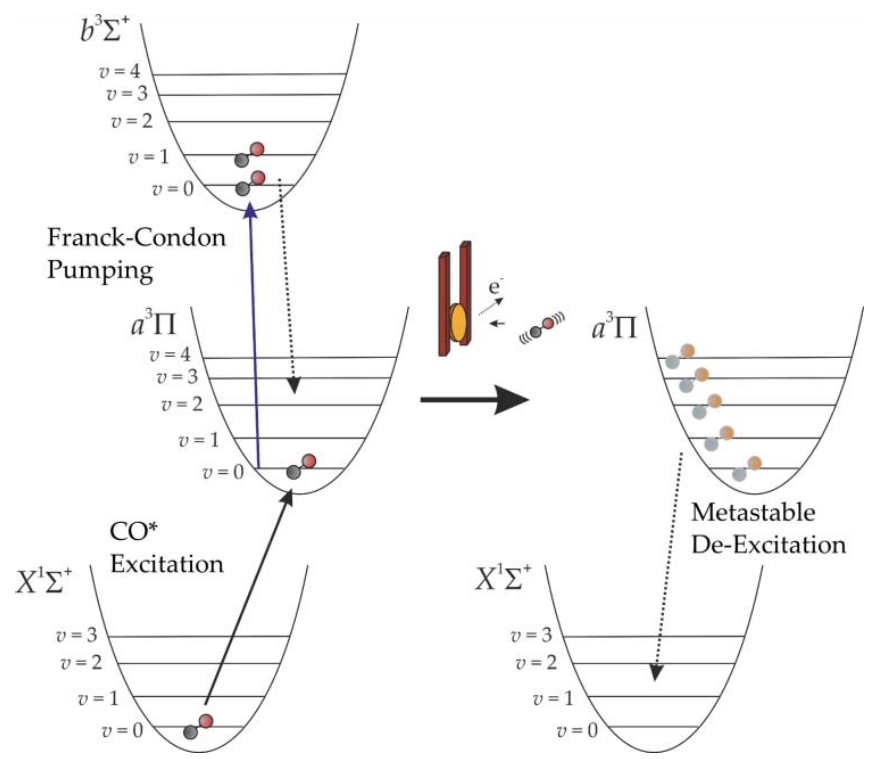

Figure 44 Schematic diagram of Franck-Condon preparation of vibrationally excited $\mathrm{CO}^{*}$ molecules. After initial excitation and velocity manipulation, $\mathrm{CO}^{*}(v=0)$ is excited to $\mathrm{b}^{3} \Sigma^{+}(v=0$ or 1$)$. $\mathrm{CO}^{*}(v>0)$ is populated according to Franck-Condon factors via spontaneous emission. $^{94}$

Electron emission signals were also recorded at different $\mathrm{CO}^{*}$ incident velocities following a similar procedure to that outlined above. We obtained a reference time-of-flight trace of the molecular beam pulse by scanning the delay time between the excitation and ionization lasers. We then normalized electron emission to the number of incoming metastable molecules by comparing the ion time-of-flight profile to the electron time-of-flight profile from the surface. For all measurements, the finite lifetime of the $a^{3} \Pi$ state has been considered and the signals have been corrected accordingly.

Figure 45 shows the enhanced probability of electron emission into vacuum as $\mathrm{CO}^{*}$ $(v>0)$ quenches on $\mathrm{Au}(111)$ over $\mathrm{CO}^{*}(v=0)$ quenching on the same surface. The lower panel shows the integrated electron emission signal recorded as the FCP laser wavelength is scanned. Enhancement clearly occurs at wavelengths corresponding to known transitions ${ }^{122}$ in the $\mathrm{b}^{3} \Sigma^{+}(v) \leftarrow \mathrm{a}^{3} \Pi(v=0)$ system. $\mathrm{b}^{3} \Sigma^{+}(v=1)$ is shown in the red scan and $\mathrm{b}^{3} \Sigma^{+}(v=0)$ in the blue scan. The transitions correspond to the 1+1 REMPI spectra shown in the upper panel. For the REMPI scans, the laser intensity was increased by focusing with $f=500 \mathrm{~mm}$ lens and increasing laser energy to $1 \mathrm{~mJ} /$ pulse. 


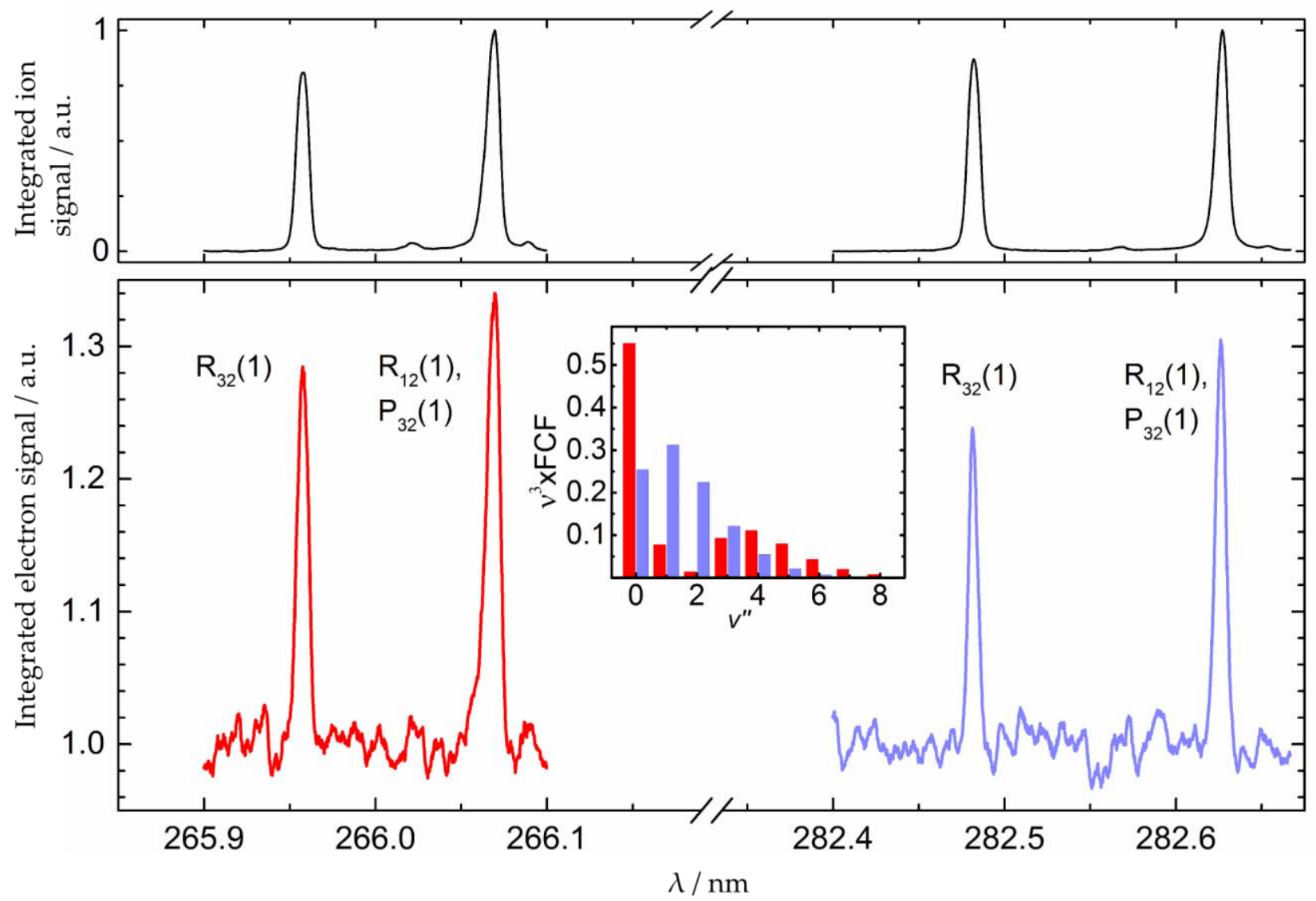

Figure 45 Lower panel: integrated electron emission signal plotted versus FCP laser wavelength. Transitions to $\mathrm{b}^{3} \Sigma^{+}(v=0)$ (red, left) and $\mathrm{b}^{3} \Sigma^{+}(v=1)$ (blue, right) enhance electron emission probability. Upper panel: simultaneously recorded REMPI ion signals. Inset: the $\mathrm{CO}^{*}$ vibrational population distributions resulting from the excited molecules relaxing back to the $a^{3} \Pi$ electronic state. Vibrational populations are calculated based on published FranckCondon factors. ${ }^{122}$ The spectra are recorded under conditions of saturation for the $b^{3} \Sigma^{+} \leftarrow a^{3} \Pi$ transitions. Note that because the two peaks in each spectrum consist of a different number of rovibrational transitions, the laser excitation efficiency is $1 / 2$ for the smaller peak and $2 / 3$ for the larger peak. When scaled for excitation efficiency, the resulting enhancement is the same for FCP pumping via both transitions.

The weaker of the two peaks shown in each spectrum of Figure 45 occurs due to the $R_{32}(1)$ transition and the stronger peak due to the non-resolved $P_{32}(1)$ and $R_{12}(1)$ transitions. Assuming each transition is saturated, 2/3 of all molecules will be excited to the $\mathrm{b}^{3} \Sigma^{+}$state at the center of the stronger peak. The enhancement, $\varepsilon$, for a given population of vibrationally excited CO* produced by FCP is defined by $\gamma_{v i b}=\varepsilon \gamma_{0}$, where $\gamma_{0}=0.13$ is the electron emission probability of $\mathrm{CO}^{*}(v=0)$ quenching on $\mathrm{Au}(111)$ and $\gamma_{\mathrm{vib}}$ is the electron emission 
probability for vibrationally excited molecules. The subscript vib indicates the vibrational quantum number. $1 / 3$ of all $\mathrm{CO}^{*}$ in the FCP laser volume are not laser excited and their enhancement is unity. Measured enhancement, $\varepsilon_{\mathrm{m}}$, is related to actual enhancement due to vibrationally excited molecules by the relation $1 / 3+2 \varepsilon / 3=\varepsilon_{\mathrm{m}} \Rightarrow \varepsilon=3 \varepsilon_{\mathrm{m}} / 2-1 / 2$. This analysis yields an enhancement of $\varepsilon=1.47$ and $\varepsilon=1.51$ for $\mathrm{CO}^{*}$ pulses pumped via the $\mathrm{b}^{3} \Sigma^{+}(v=0)$ and $\mathrm{b}^{3} \Sigma^{+}(v=1)$ transitions, respectively.

Observed enhancement factors necessarily reflect averages over the entire vibrational state distributions, shown in the inset of Figure 45. Clearly, the population in $\mathrm{a}^{3} \Pi(v=1-3)$ is much smaller and the population $\mathrm{a}^{3} \Pi(v=0)$ much higher for those molecules prepared via the $\mathrm{b}^{3} \Sigma^{+}(v=1)$ state. Despite this, the enhancement factors for beam pulses prepared via the two different states are comparable. Thus, the contribution to electron emission from states with $v \geq 4$ must be much larger than for $v=1-3$. The enhancement relative to the $v=0$ state can be calculated by solving a system of coupled linear equations. ${ }^{48}$ In this way, emission yields of $\gamma_{1-3}=(1.48 \pm 0.14) \gamma_{0}$ and $\gamma_{\geq 4}=(2.59 \pm 0.39) \gamma_{0}$ have been calculated. While state-specific results are impossible to calculate from these results, the analysis demonstrates strong enhancement of electron emission with increasing vibrational quantum number.

The dependence of incidence translational energy was also studied, as shown in Figure 46. Within the uncertainty of the measurements, electron emission probability is independent of the incident molecules' velocity over the range studied. 


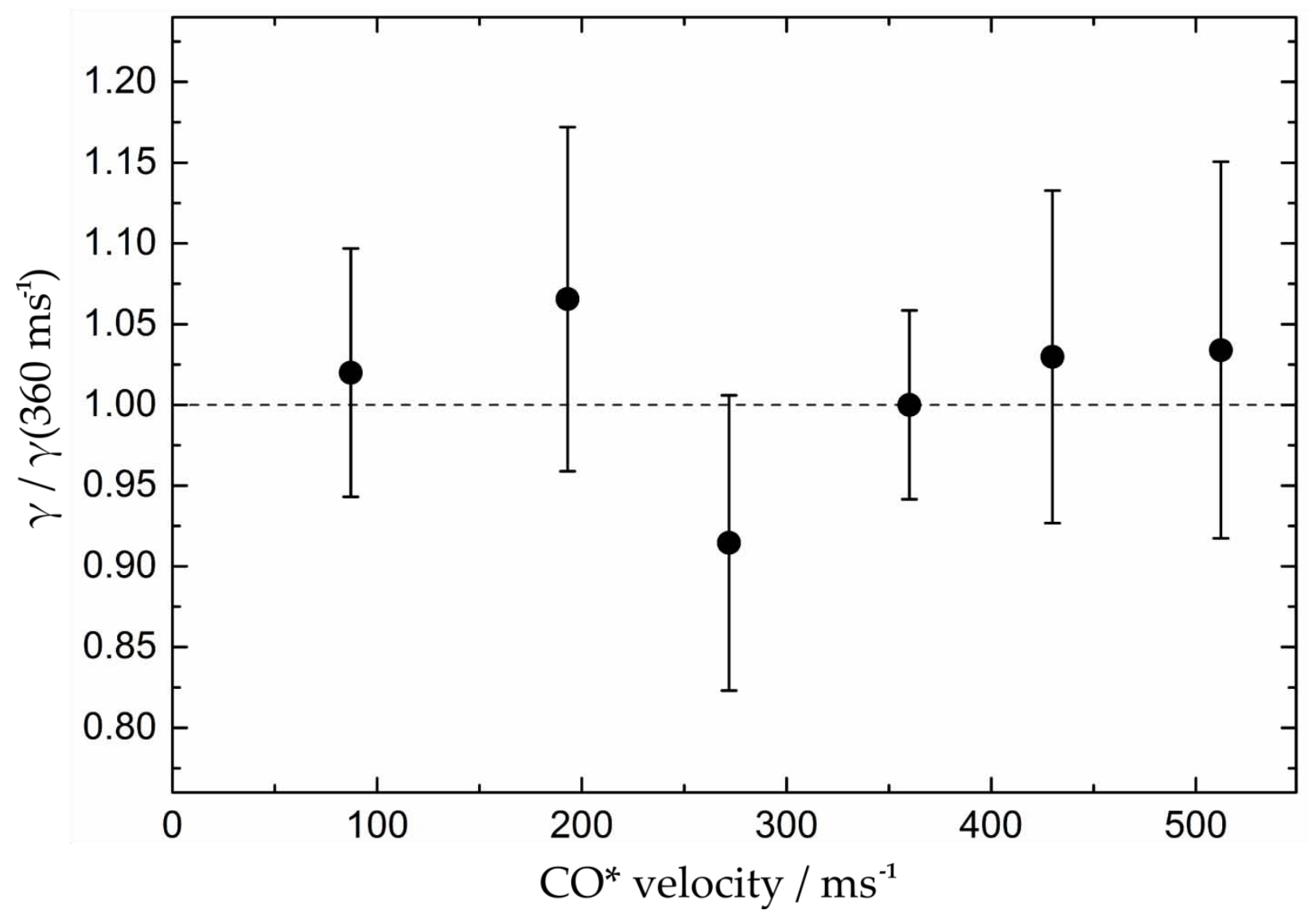

Figure 46 Electron emission from $\mathrm{CO}^{*}$ at different incident velocities quenching on $\mathrm{Au}(111)$. Emission probability is given in units of $\gamma_{0}$ for $360 \mathrm{~m} / \mathrm{s}$ molecules $(0.13)$. Electron emission probability has no measurable dependence on incident kinetic energy over the range of velocities studied.

The large uncertainty in the measurements shown in Figure 46 precludes assignment of any translational energy dependence over the range studied. It should be noted that the experiments for different incidence translation energies have been performed by determining the emission probability for each incident velocity individually. Because a variety of experimental parameters were changed between measurements, the time between consecutive measurements was long. Scanning the beam's velocity and the timings of detection lasers directly using an electronic computer would be preferable and is currently implemented in the apparatus. This will allow us to resolve any trends in the translational energy dependence of electron emission probability. 


\subsubsection{Discussion}

In this work, we scatter $\mathrm{CO}^{*}$ with orbital configuration $1 \sigma^{2} 1 \sigma^{* 2} 2 \sigma^{2} 2 \sigma^{* 2} 1 \pi^{4} 3 \sigma^{1} 1 \pi^{* 1}$ to a $\mathrm{Au}(111)$ surface. As the molecule approaches the surface, it interacts with the surface and relaxes to the ground state with the orbital configuration $1 \sigma^{2} 1 \sigma^{* 2} 2 \sigma^{2} 2 \sigma^{* 2} 1 \pi^{4} 3 \sigma^{2}$ with the possibility to emit an electron into vacuum. The probability of electron emission is significantly enhanced with vibrational excitation of the molecule. Zubek suggested that vibrational enhancement of electron emission probability can be described within the AD mechanism by assuming that electron emission is proportional to $E_{\text {ex }}$ summed over all possible Franck-Condon weighted molecular decay channels. ${ }^{123}$ See Figure $47 . \mathrm{AD}$ is an electronic transition and therefore fast. Therefore molecular vibrational transitions must reflect Franck-Condon factors. This model predicts that $\gamma_{1-3} / \gamma_{0}=1.02$ and $\gamma_{\geq 4} / \gamma_{0}=1.10$, significantly underestimating the experimentally observed enhancement. ${ }^{94}$

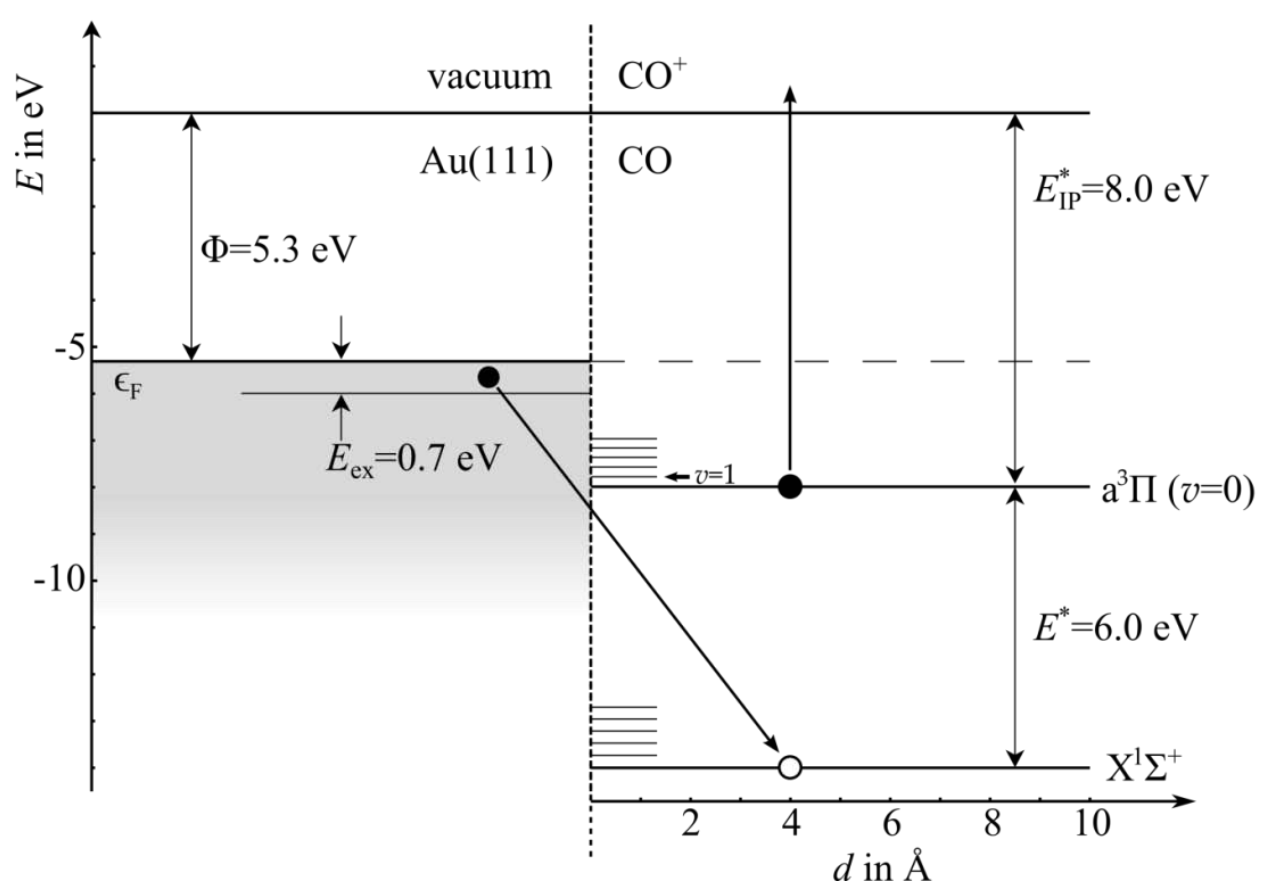

Figure 47 Energy diagram of metastable $\mathrm{CO}^{*}$ quenching at the $\mathrm{Au}(111)$ surface via Auger deexcitation. An electron from near the metal's Fermi level fills the $3 \sigma$ orbital $\left(\mathrm{X}^{1} \Sigma^{+}\right)$, simultaneously ejecting the $1 \pi^{*}$ electron $\left(\mathrm{a}^{3} \Pi\right)$. With increasing initial vibrational quantum number, $E^{*}$ and $E_{\text {ex }}$ increase and electron emission probability should increase commensurately. This analysis severely underestimates the magnitude of enhancement. See text for more discussion. 
During AM de-excitation, on the other hand, an electron first tunnels from the surface to the molecule, forming $\mathrm{CO}^{-}$, with orbital occupation $1 \sigma^{2} 1 \sigma^{* 2} 2 \sigma^{2} 2 \sigma^{* 2} 1 \pi^{4} 3 \sigma^{2} 1 \pi^{* 1}$. The $\pi^{*}$ electron is subsequently auto-detached on a time-scale commensurate with one molecular vibrational period. The finite lifetime of the anion is due to the dependence of the electron's binding energy to $\mathrm{CO}$ being dependent on both the molecule-surface distance and the $\mathrm{C}-\mathrm{O}$ bond length.

The ground state of $\mathrm{CO}^{-}$is known to possess ${ }^{2} \Pi$ symmetry and lie $1.7 \mathrm{eV}$ above the ground state of $\mathrm{CO} .^{124-127}$ At long surface distances, the electron affinity of $\mathrm{CO}^{*}$ is $4.3 \mathrm{eV}$, whereas the $\Phi_{\mathrm{Au}(111)}=5.3 \mathrm{eV}$. Hence, electron transfer from the surface to the molecule cannot occur at long surface-molecule distances. The upper panel of Figure 48 shows a potential energy versus molecule bond length curve for $\mathrm{CO}^{*}$ and an electron at the Fermi level of the metal (red solid) and for the $\mathrm{CO}$ anion and a singly ionized gold surface (i.e. after electron transfer has occurred, blue dashed) at asymptotic molecule-surface distance. Electron transfer from surface to molecule is represented by a transition from the red curve to the blue. At large molecule-surface distances, electron transfer is energetically forbidden. As the molecule approaches the surface, the ionic state is stabilized by image charge interaction. ${ }^{117}$ At $\approx 5.4 \AA$, a distance where electron transfer is expected to be efficient, ${ }^{26,}{ }^{128} \mathrm{CO}^{-}$is isoenergetic with $\mathrm{CO}^{*}$ at stretched bond distances. The middle panel of Figure 48 shows curves analogous to the top panel, but with the molecule $5.4 \AA$ from the surface. Resonant ionization at a metal surface first becomes possible for a stretched $\mathrm{CO}$ molecule, where tunneling to the molecule may occur for electrons originating at the Fermi level of the surface. 

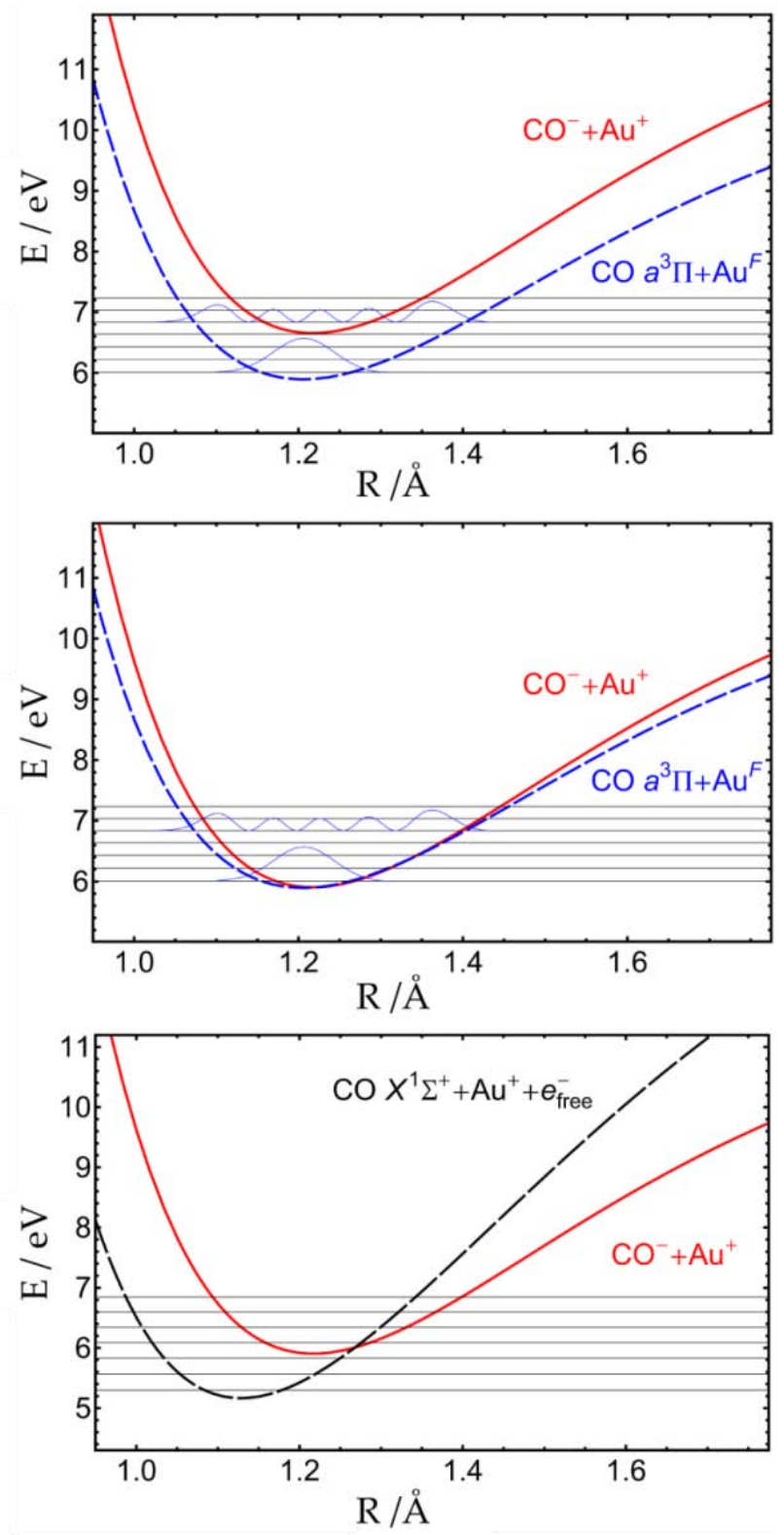

Figure 48 Upper panel: potential energy curves for the $\mathrm{CO} / \mathrm{Au}(111)$ system as a function of internuclear distance of $\mathrm{CO}$ with molecule positioned at asymptotic distance from the surface. The dashed blue curve describes vibration of $\mathrm{CO}^{*}$ with an electron at the Fermi level of the metal. Vibrational energy levels of the $\mathrm{CO}^{*}$ are represented by horizontal lines. The solid red curve represents the vibration of $\mathrm{CO}^{-}$after electron transfer from the metal. At asymptotic molecule-surface distances, electron transfer is energetically forbidden. Middle panel: similar curves to upper panel, but molecule-surface distance is $5.4 \AA$. Image charge forces at this distance lower the energy of the anionic state (red curve) by $0.75 \mathrm{eV}$. Electron transfer can now occur for electrons at the Fermi level at $\mathrm{C}-\mathrm{O}$ bond distances greater than $1.2 \AA$. Vibrational energy levels of the $\mathrm{CO}^{*}$ are represented by horizontal lines. Lower panel: the red curve is the same as the middle panel. The dashed black curve represents the vibration of the $\mathrm{CO}$ ground state with an electron at the vacuum level. Horizontal lines represent vibrational energy levels of ground state $\mathrm{CO}$. 
The AM model also provides an explanation for vibrational enhancement of electron emission probability. The lower panel of Figure 48 shows potential energy curves of the $\mathrm{CO}$ neutral ground electronic state (black dashed) and anion (solid red) at $5.4 \AA$ molecule-surface distance. The dashed black dashed curve is shifted by $5.3 \mathrm{eV}$ because the electron initially at the metal's Fermi level is now at the vacuum level. The anion is more stable than the neutral molecule only at extended bond lengths. Hence, if an electron is transferred from the metal to the metastable molecule near the outer classical turning point of vibration, as the middle panel of Figure 48 suggests, the electron will be ejected from the molecule near the inner classical turning point. The lifetime of the anion is therefore expected to be on the order of half a vibrational period, $\approx 10^{-14} \mathrm{~s}^{-1}$. The lifetime of the resonance increases with increasing vibrational quantum number as the molecule spends more time at extended $\mathrm{CO}$ bond lengths. It should be noted that the large electron emission probability seen in this work is consistent with that seen in vibrationally promoted electron emission, a mechanism involving similar electron transfer. ${ }^{27}$ All curves in Figure 48 are taken from published sources. ${ }^{125,126}$

Although energetically accessible, the Auger de-excitation mechanism proceeds via a simultaneous two electron step, whereas anion mediated auto-detachment consists of two single electron steps. It can, therefore, be expected that the anion mediated mechanism dominates deexcitation. Additionally, the anion mediated mechanism involves electron transfer from the metal to an excited electron orbital of the molecule, whereas the Auger de-excitation involves electron transfer to a more compact ground state orbital. Consideration of the overlap of the molecular orbitals with the wave functions of the metal suggests that the anion will be formed at surface distances for which Auger de-excitation is not efficient.

The CO* molecule must reach a distance of 5.0-5.4 $\AA$ from the $\mathrm{Au}(111)$ surface for resonant electron transfer from the Fermi level of the metal surface to the molecule to become possible. Electron transfer is more efficient at stretched C-O internuclear bond distances and ET can be expected to occur when the molecule is near its outer classical turning point. Within $\approx 10$ fs, the CO bond is compressed and the electron is released. The extremely short lifetime of the resonance precludes translational energy dependence over the range studied. On a fs timescale, motion of the molecule toward the surface within the timeframe of both ET steps is negligible. 


\subsubsection{Electron emission from adsorbate covered $\mathrm{Au}(111)$ surfaces}

The experiments discussed in the previous section showed that the quenching of $\mathrm{CO}^{*}$ at a $\mathrm{Au}(111)$ surface is incompatible with an Auger de-excitation (AD) mechanism. ${ }^{83}$, $94 \mathrm{An}$ alternative anion mediated (AM) mechanism was suggested. In contrast to an AD quenching mechanism, AM de-excitation proceeds via two separate one electron steps. First, an electron transfers from the surface to the molecule to form an anion. Second, the ion auto-detaches an electron which can be either emitted into vacuum (and detected) or absorbed by the surface. The measured electron emission probability depends on both steps: the electron transfer efficiency from the surface to the metastable and the subsequent electron emission efficiency.

The probability of electron transfer from metal surface to molecule and the lifetime of the resulting anion are both strongly dependent on the distance between molecule and surface. IN this section, I manipulate the distance of closest approach between the surface and the molecule by controlled deposition of rare gas adsorbates. The introduction of rare gas spacers has been successfully implemented by Hotzel et al. to probe the effect of surface-molecule distance in two photon photoemission (2PPE) studies. This introduction of a "spacer layer," however, not only influences the range of possible interaction distance, it can also lead to significant change of the surface work function. ${ }^{129}$ Very little literature exists describing the influence of the spacer layer on electron emission efficiency.

In the following section, the influence of surface adsorbate coverage on the de-excitation process for the $\mathrm{CO} / \mathrm{Au}(111)$ system is systematically probed by monitoring the electron emission probability of the quenching process as a function of controlled adsorption of $\mathrm{Ar}, \mathrm{Kr}$, and $\mathrm{Xe}$ on the target $\mathrm{Au}(111)$ surface. In this way the interaction distance between the molecule and the metal surface can be directly controlled. It would be reasonable to expect the electron emission yield to decrease with increased spacer layer thickness due to decreased overlap between electron wave functions of molecule and surface at increased surface distance. This effect, however, is only observed for thick over-layers. Interestingly, the electron emission yield increases markedly with adsorbed rare gas monolayers at the surface. By careful adjustment of adsorbate coverage, electron emission probability can be brought to near unity for $\mathrm{Ar}$ and $\mathrm{Kr}$ adsorbates. This behavior is explained by an enhanced probability of reflection at the surface due to rare gas adsorption. 


\subsubsection{Experimental and results}

The effect of rare gas adsorbate coverage on the electron emission probability of $\mathrm{CO}^{*}$ quenching on $\mathrm{Au}(111)$ were performed in the UHV surface scattering apparatus described earlier

in this work. ${ }^{130}$ The molecular beam is prepared identically as in the experiments described in the previous sections with no ionization step. The surface is cleaned by standard cycles of sputtering with $\mathrm{Ne}^{+}$ions and annealing at $1000 \mathrm{~K}$. The experiments make use of the knowledge deduced from temperature programmed desorption (TPD) experiments (section 3.1) to change the composition of the scattering surface. Briefly, the cold surface is dosed with thick overlayers of argon, krypton, or xenon. The adsorbate covered surface is then placed in the path of the beam of metastable $\mathrm{CO}^{*}$ molecules and electron emission signal is recorded as a function of temperature. Adsorbate coverage during the surface scattering experiments is determined by correlating the surface temperature to existing TPD spectra for similar adsorbate doses and heating rate. Surface coverage is determined by integrating the TPD spectra and normalizing to the monolayer signal.

It is known from low energy electron diffraction (LEED) experiments that xenon binds at low coordination sites. ${ }^{131-133}$ No explicit study of the binding geometry of argon or krypton on the $\mathrm{Au}(111)$ surface could be found in the literature. For $\mathrm{Ar}$ and $\mathrm{Kr}$ on $\mathrm{Au}(111)$ we assume structures similar to $\operatorname{Ar} / \operatorname{Ag}(111)$ and $\mathrm{Kr} / \mathrm{Ru}(0001)$ which have been determined experimentally. ${ }^{134}$ This is a reasonable assumption, as desorption temperatures are similar, indicating adsorption dominated by dispersion forces on close packed transition metal surfaces. These monolayer adsorbate structures are shown in Figure 49. 


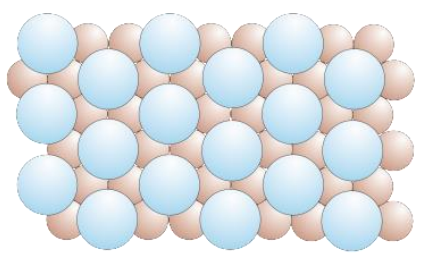

$\mathrm{Au}(111)-(\sqrt{ } \mathrm{x} \backslash \mathrm{3}) \mathrm{R} 30^{\circ}-\mathrm{Xe}$

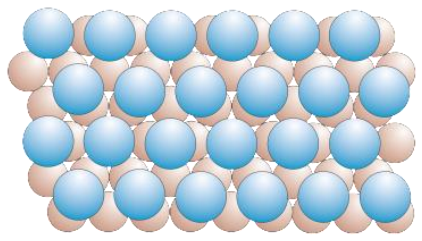

$\operatorname{Au}(111)-(3 \times 3)-4 K r$

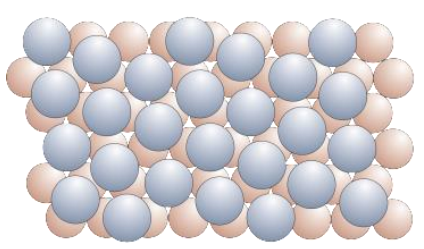

$\operatorname{Au}(111)-(\backslash 7 \times \backslash 7) R 19.1^{\circ}-4 \mathrm{Ar}$

Figure 49 Assumed adsorption geometry for xenon, krypton and argon on a $\mathrm{Au}(111)$ surface. Due to lack of experimental data, we assume argon and krypton adsorption to be comparable to the adsorption geometry on similar close-packed transition metal surfaces.

We increase the distance of closest approach between the metal surface and incoming molecule by adsorption of further rare gas layers on top of the monolayer. The rare gases crystalize in a closest packed structure, ${ }^{135}$ allowing calculation of surface distance induced by the rare gas spacers using their van der Waals radii. See Table 4. This precise knowledge of surface coverage is used to interpret electron emission measurements resulting from $\mathrm{CO}^{*}$ scattering from adsorbate decorated surfaces. 
Table 4 Increased distance between metal surface and impinging molecule induced by adsorption of rare gas spacers. The distances have been calculated using van der Waals radii between the atoms' center of mass and assuming close-packing of the rare gases.

\begin{tabular}{|c|c|}
\hline \multicolumn{2}{|c|}{ Argon } \\
\hline $1^{\text {st }}$ layer & $3.76 \AA$ \\
\hline $2^{\text {nd }}$ layer & $6.54 \AA$ \\
\hline $3^{\text {rd }}$ layer & $9.32 \AA$ \\
\hline \multicolumn{2}{|c|}{ Krypton } \\
\hline $1^{\text {st }}$ layer & $4.04 \AA$ \\
\hline $2^{\text {nd }}$ layer & $7.03 \AA$ \\
\hline $3^{\text {rd }}$ layer & $10.01 \AA$ \\
\hline \multicolumn{2}{|c|}{ Xenon } \\
\hline $1^{\text {st }}$ layer & $4.32 \AA$ \\
\hline $2^{\text {nd }}$ layer & $7.52 \AA$ \\
\hline $3^{\text {rd }}$ layer & $10.71 \AA$ \\
\hline
\end{tabular}

The influence of rare gas adsorption on the electron emission induced when $\mathrm{CO}^{*}$ quenches on $\mathrm{Au}(111)$ is shown in Figure 50. For these experiments, the $19 \mathrm{~K}$ surface was first dosed with a well-defined exposure of rare gas to produce $>5$ ML coverage. The temperature of the target crystal was then raised at a constant rate while the electron emission resulting from a constant flux of $\mathrm{CO}^{*}$ is detected on an MCP. The exoelectron signal is shown in panels $\mathrm{b}, \mathrm{d}$, and $\mathrm{f}$ as a function of surface temperature. In a separate series of experiments, conventional TPD is performed with the same initial dosage and heating rate.

Two obvious features are clear in each electron emission spectrum. First, all measurements show the same electron yield at the highest surface temperatures, i.e., once the surface is free of adsorbates, electron emission is always that for the $\mathrm{CO} * / \mathrm{Au}(111), \gamma=0.13 \pm$ $0.05{ }^{48,83}$ This value is used to set the $y$-axis scale in panels $b, d$, and $f$. The second obvious feature is that no electron emission is seen at the lowest temperatures indicating that enough layers of rare gas adsorbate suppress electron emission completely. 


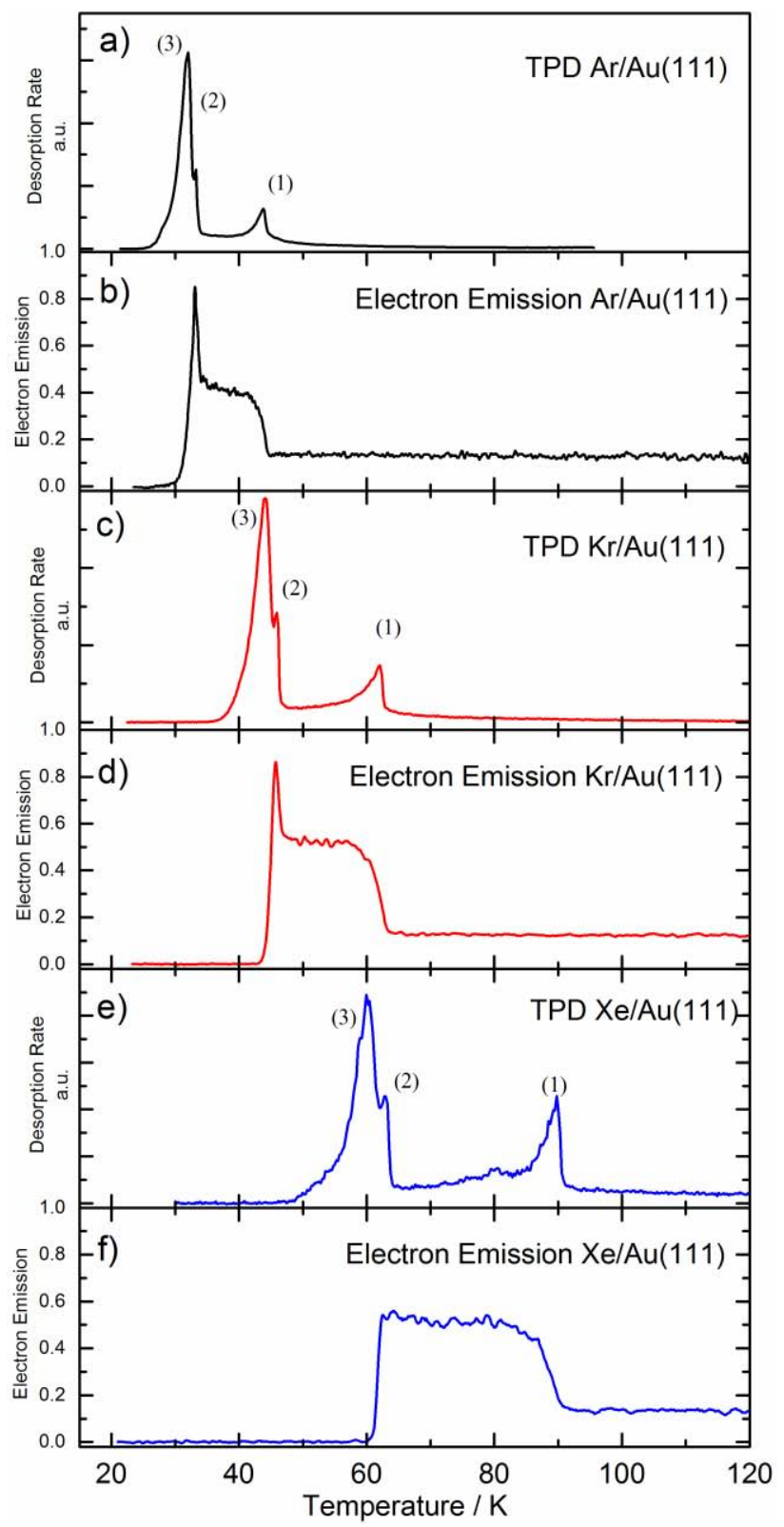

Figure 50 TPDs (a,c,e) and electron emission curves (b,d,f) for Au(111) prepared with argon, krypton, and xenon adsorbates. Heating rate was $10 \mathrm{~K} \mathrm{~min}^{-1}$ for all scans and initial coverage was $>5$ ML. For all TPDs, (3) indicates the overlayer peak, (2) the bilayer peak and (1) the monolayer peak. The changes in the electron emission curves can be directly correlated to desorption of adsorbate layers from the surface.

A clear picture of the influence of rare gas adsorption on electron emission probability can be gained by comparing the temperature dependence of the electron spectra to the TPD spectra. In describing the adsorbate layers we refer to: (1) the monolayer, the $1^{\text {st }}$ layer bound directly to the metal, (2) the bilayer, which is bound to the monolayer, and (3) overlayers, which 
are the rest of the adsorbed gas. The TPD spectra of all adsorbates exhibit three clear features, labeled (1), (2), and (3). The overlayer desorption peaks (3) are seen at the lowest surface temperature and are due to desorption of the most weakly bound outer adsorbate layers. Feature (2) represents the more strongly bound $2^{\text {nd }}$ layer and feature (1) indicates desorption of the monolayer. For all three rare gases, electron emission appears only as the $2^{\text {nd }}$ layer begins to be exposed, at $\mathrm{T}>$ (3). For $\mathrm{Ar}$ and $\mathrm{Kr}$ adsorbates, an abrupt decrease in electron emission probability occurs as the adsorbate bilayer sublimates, exposing the monolayer. This shows how sensitive the electron emission process is to the atomic structure of the surface.

In order to derive quantitative information about the adsorbate coverage dependence of $\gamma$, instantaneous coverage at a given temperature was determined by integrating under a TPD scan and normalizing to the integral under a monolayer. By correlating the instantaneous coverage with the electron emission for the corresponding surface temperature, the electron emission probability is determined as a function of coverage as can be seen in Figure 51.

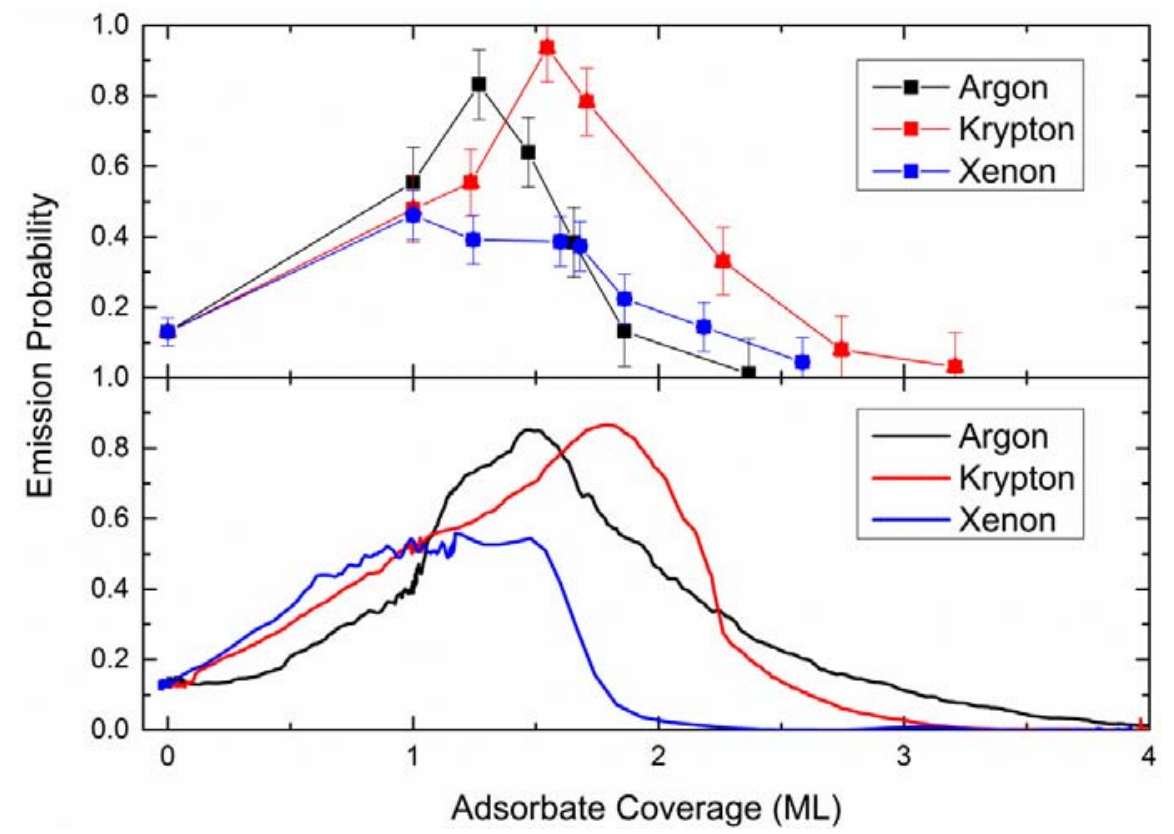

Figure 51 Upper panel: electron emission probability versus adsorbate coverage for argon, krypton and xenon. Lines are added to guide the eye. The error bars reflect the uncertainty calculated from multiple measurements of monolayer enhancement as presented in Table 5. Lower panel: electron emission probability versus coverage calculated by correlating a temperature programmed electron emission scan with a TPD spectrum for each adsorbate species. For more details, see text. 
Electron emission probability was measured from several surfaces prepared with systematically varied adsorbate coverages of argon, krypton, and xenon in order to experimentally confirm the validity of the analysis presented in the lower panel of Figure 51. For each data point, electron emission was first measured from a clean surface; afterwards, the surface was dosed with adsorbates by backfilling the chamber with the adsorbate gas. Electron emission was then measured immediately in order to minimize drift in pulsed valve performance, laser power, etc. between measurements. After performing each set of two electron emission measurements, surface coverage was determined with a TPD scan, thereby removing the coverage. For this reason each experiment determines the electron emission probability at only a single coverage. Results of these experiments are shown in the upper panel of Figure 51. Clearly, the experimental data yields similar results, lending credence to our analysis.

Absolute electron emission yields are remarkably high for all adsorbate species. For argon and krypton covered surfaces, emission probability approaches unity between one and two monolayers of coverage. Xenon covered $\mathrm{Au}(111)$ does not exhibit further enhancement at coverages above one monolayer. Monolayer emission probability is summarized in Table 5

Table 5 Absolute electron emission probability for $\mathrm{CO}^{*}$ quenching on monolayer covered $\mathrm{RG} / \mathrm{Au}(111) . \mathrm{RG}=\mathrm{Ar}, \mathrm{Kr}, \mathrm{Xe}$.

\begin{tabular}{|c|c|}
\hline Adsorbate & Emission probability \\
\hline $\mathrm{Ar}$ & $0.56 \pm 0.10$ \\
\hline $\mathrm{Kr}$ & $0.48 \pm 0.10$ \\
\hline $\mathrm{Xe}$ & $0.46 \pm 0.07$ \\
\hline
\end{tabular}

Adsorption of rare gas also induces a change in the work function, $\Phi$, of the surface. ${ }^{129}$ Because the anion mediated de-excitation mechanism of $\mathrm{CO}^{*}$ at a $\mathrm{Au}(111)$ surface depends on the potential energy of all involved orbitals, a careful consideration of the influence of changing work function is necessary. We measured the working function using the scanning Kelvin probe introduced in section 2.2.3.2. The change in $\Phi$ of $\mathrm{Au}(111)$ as a function of adsorbate coverage is presented in Figure 52. The measured work function change is in good agreement with previous measurements. ${ }^{129}$ The work function change induced by monolayer coverage of Ar, Kr, and Xe is 180,250 , and $400 \mathrm{mV}$, respectively. This is expected because due to the increasing polarizability of the noble gases going down the periodic table. Xenon atoms induce a larger 
surface dipole moment than $\mathrm{Kr}$ and $\mathrm{Ar}$ atoms, leading directly to a larger work function change. Adsorption of a second and further adsorbate layer results in only minor changes to $\Phi$.

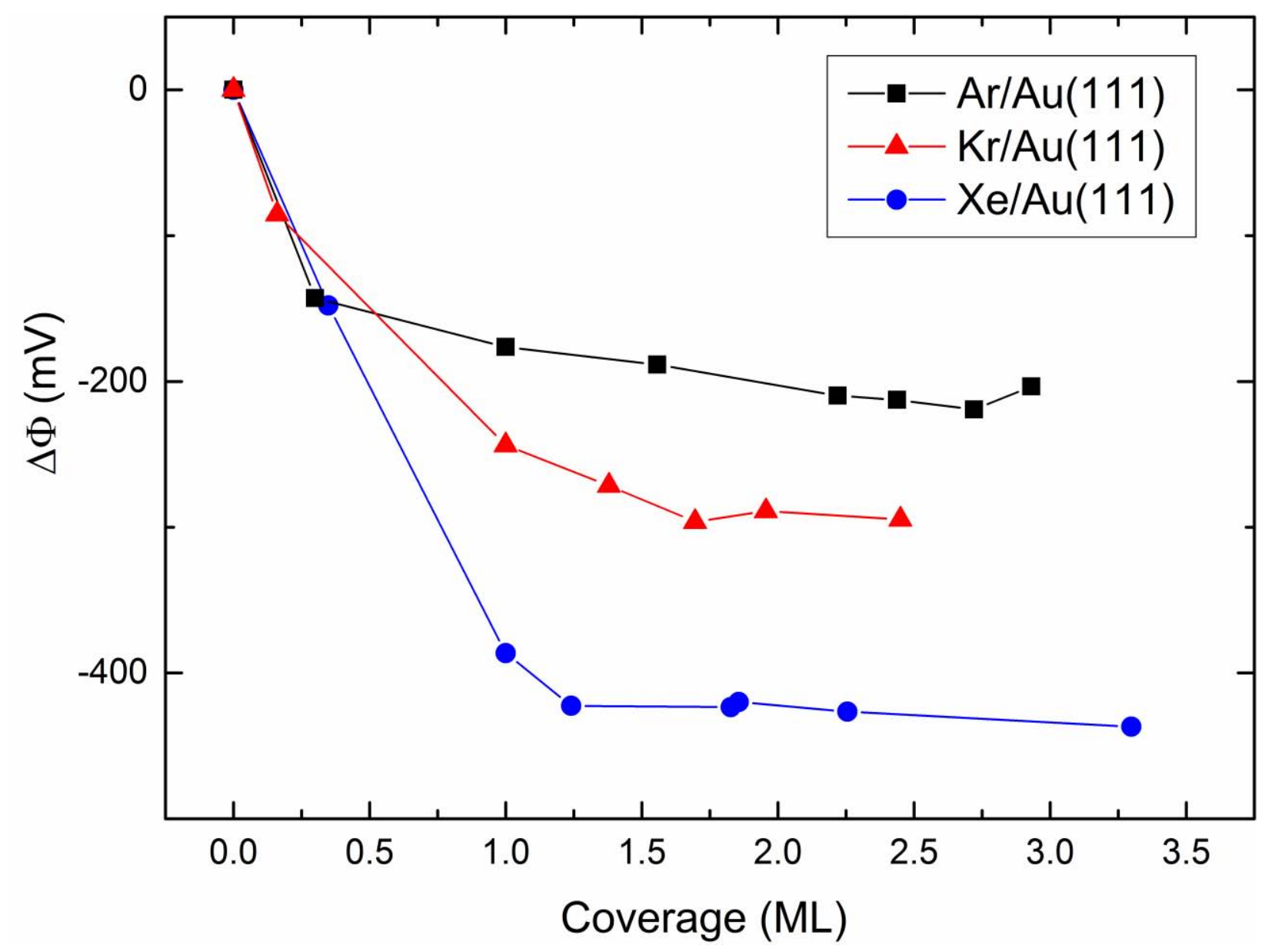

Figure 52 Work function, $\Phi$, change of the $\mathrm{Au}(111)$ surface due to the adsorption of rare gases. $\Phi$ drops sharply with the adsorption of one monolayer. The effect of any further adsorbed gas is minor. Lines are drawn to guide the eye.

\subsubsection{Discussion}

The influence of rare gas adsorption on electron emission is discussed based on the electron transfer mediated auto-detachment mechanism described in detail in Section 3.2.1 ${ }^{94,112-}$ 115 According to this mechanism, electron emission proceeds in two steps. In the first step, an electron is transferred from the surface to the molecule when the molecule's bond extended beyond the equilibrium bond distance. The electron is transferred from the surface to a short lived anionic shape resonance lying just above the ground state. The electron is subsequently 
emitted from the molecule after recompression of the bond, leaving the $\mathrm{CO}$ molecule in its ground state and the excess electronic excitation in the emitted electron as discussed in detail in the previous section. For a complete discussion of the influence of adsorbates on $\gamma$, two main points must be addressed, namely the influence of the adsorbate-induced work function change on the initial electron transfer (ET) step from the surface to the molecule and the effect of surface morphology on the mechanism's second step. Electron emission via Penning ionization of the adsorbate is precluded due to the high ionization energy of the rare gases.

Because the de-excitation of $\mathrm{CO}^{*}$ depends on the work function of the target surface, any work function change might also affect the de-excitation probability. ${ }^{112-114}$ Reducing the work function directly influences the first step of the AM mechanism, i.e. ET from the surface to the metastable molecule. Due to the lower energy barrier between electrons at the Fermi level and the orbitals of the molecule, tunneling to the incident molecules becomes likely a longer molecule-surface distances for low work function surfaces, as shown in Figure 53. ${ }^{113}$ Despite this, the expected phenomenon cannot explain the experimental results. The change in work function scales with the polarizability of the adsorbate: $\mathrm{Xe}>\mathrm{Kr}>\mathrm{Ar}$, as shown in Figure 52. The measured monolayer electron emission probability relative to the clean surface shows the opposite trend: $\mathrm{Ar}>\mathrm{Kr}>\mathrm{Xe}$, as can be seen in Table 5.

Figure 52 also shows that adsorption of a second atomic layer decreases the work function only slightly compared with the monolayer induced shift for all three adsorbates. In contrast, the electron emission signal for $\mathrm{Ar}$ and $\mathrm{Kr}$ covered $\mathrm{Au}(111)$ increases by a factor of 1.8 over the monolayer signal as coverage increases over one monolayer. See Figure 51. Based on these observations, we conclude that the adsorbate induced change of the work function cannot explain the observed enhancement in the electron emission probability when scattering $\mathrm{CO}^{*}$ from adsorbate covered $\mathrm{Au}(111)$ surfaces. 


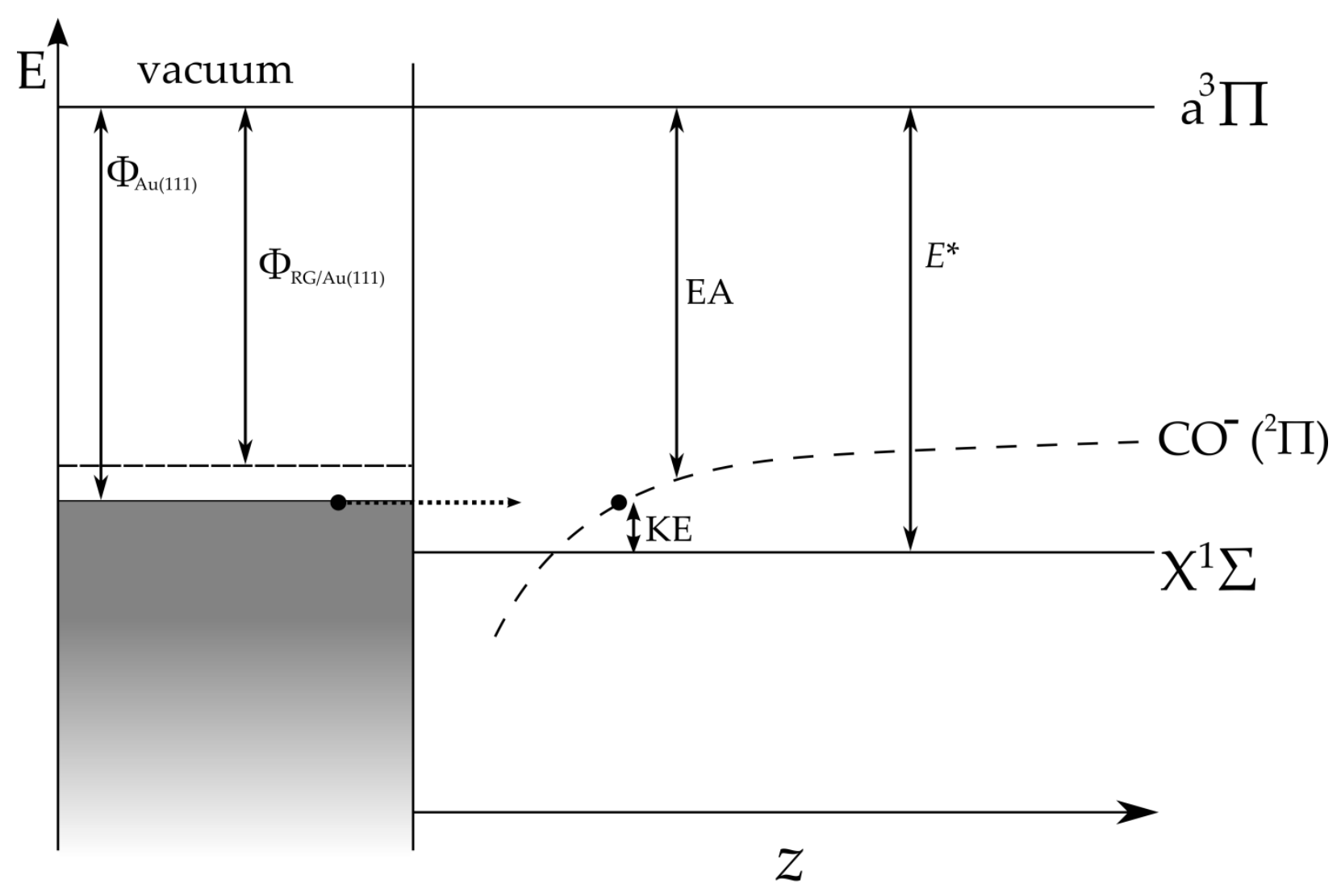

Figure 53 Anion mediated de-excitation mechanism for the case of $\mathrm{CO}^{*}$ quenching on clean and rare gas (RG) adsorbate covered $\mathrm{Au}(111)$. While a decrease in the surface work function can be expected to result in increased electron emission, $\Delta \Phi$ cannot completely explain the effect of rare gas (RG) adsorbates on the magnitude of $\gamma$.

More probable is that the increased probability of electron emission results from an increased efficiency of electron emission from the molecule into vacuum. After ET from the surface to the molecule has occurred, the short-lived shape resonance ( $\tau \approx 10 \mathrm{fs}$ ) decays to the ground electronic state of the neutral molecule by emitting an electron. This occurs at distances larger than $5 \AA .{ }^{94}$ Assuming anisotropic emission of electrons from the anion in front of the surface, simple statistical considerations based on the planar symmetry of the system suggest that half of the auto-detached electrons will be emitted toward vacuum and half toward the surface. This simple picture ignores any possible image forces which may act on the emitted electrons. 
Electrons emitted in the direction of the surface can be either absorbed (going undetected) or reflected into vacuum, where they can be observed in this experiment.

The observations of this work support the idea that the adsorption of rare gas atoms increases the electron reflection probability of the surface. The complete absence of electron emission upon adsorption of a third atomic layer can be explained by considering the closest packed structure of the adsorbate atoms. Two layers of rare gas spacers still leave small holes through the adsorbate layer through which electron transfer can be expected to be efficient. A third adsorbate layer plugs these holes.

Low energy electron diffraction (LEED) studies of hydrogen covered tungsten surface have shown a strong surface coverage dependence on the backscattering of electrons with low kinetic energies. ${ }^{136}$ At electron energies below $3 \mathrm{eV}$ in particular, the electron reflection probability of the surface is enhanced dramatically by the presence of adsorbates. The electrons auto-detached from $\mathrm{CO}^{-}$are expected to have kinetic energy in the range of $\approx 1 \mathrm{eV}$.

The adsorbate-specific trends observed in this work can also be explained by this hypothesis. Argon, krypton, and xenon exhibit different adsorption geometries resulting from the large differences in their van der Waals radii. Figure 49 shows that argon adsorbs more densely $\left((\sqrt{7} \times \sqrt{7}) R 19.1^{\circ}-4 \mathrm{Ar}\right)$ than krypton $((3 \times 3)-4 \mathrm{Kr})$ and krypton more densely than xenon $\left((\sqrt{3} \times \sqrt{3}) R 30^{\circ}-\mathrm{Xe}\right) .{ }^{134}$ The monolayer adsorbate structures lead to a trend in how the rare gases obscure the gold surface - argon obscures the surface most completely, followed by krypton, then argon. The fraction of substrate metal surface obscured by spheres with radii corresponding to each adsorbate's van der Waals radius is: $88.1 \%(\mathrm{Ar}), 79.1 \%(\mathrm{Kr})$, and $67.8 \%$ (Xe). The trend in this quantity corresponds to the monalayer enhancement shown in Table 5. This is a further sign that altered surface reflection probability is the dominant factor influencing the observed enhancement of electron emission probability. Furthermore, this hypothesis is consistent with the observation of maximum electron emission yield for surfaces with rare gas coverages substantially above a single monolayer (Figure 51).

Interestingly, Xe adlayers induce only around half the maximum electron emission efficiency of that induced by $\mathrm{Kr}$ or Ar. Although below $1 \mathrm{ML}$ coverage the three rare gas adsorbate layers yield similar enhancements, above $1 \mathrm{ML}$ coverage xenon induces no further 
enhancement (Figure 51). Gas phase studies have shown that small clusters of xenon atoms can form stable anions when the ionizing electron polarizes the cluster's closed electronic shell. ${ }^{137}$ Accordingly, the electron affinity of equally sized clusters is significantly larger for xenon clusters than for krypton or argon clusters. ${ }^{138}$ Based on this information, we explain the exceptional behavior of xenon covered $\mathrm{Au}(111)$ by the electron affinity of the rare gas spacer: the xenon bilayer may be able to trap electrons (at least transiently) so that both ET to the CO* molecule and reflection are reduced compared to the $\mathrm{Kr}$ or Ar. This simple explanation qualitatively describes our experimental observations within the framework of the underlying AM mechanism. Studies using relatively non-reactive adsorbates with high electron affinity such as $\mathrm{SF}_{6}$ could help shed light on this hypothesis. 


\subsubsection{Conclusion}

We investigated interfacial electron transfer and charge separation by observing the exoelectrons emitted during the scattering of $\mathrm{CO}$ molecules in the $\mathrm{a}^{3} \Pi$ state $\left(\mathrm{CO}^{*}\right)$. $\mathrm{CO}^{*}$ in ground and excited vibrational states was scattered from clean $\mathrm{Au}(111)$ surface. Electron emission probability, $\gamma$, is significantly enhanced with increasing vibrational excitation of the incident molecules. The electron emission probability resulting from the de-excitation of $\mathrm{CO}^{*}$ in its vibrational ground state was found to be $\gamma_{0}=0.13 \pm 0.05$. No influence of molecular incident translational energy was observed. The electron emission yield increases with increasing vibrational excitation. $\gamma_{\mathrm{vib}}$ is $\gamma_{1-3}=(1.48 \pm 0.14) \gamma_{0}$ and $\gamma_{\geq 4}=(2.59 \pm 0.39) \gamma_{0}$.

$\mathrm{CO}^{*}$ in its ground vibrational state was also scattered from rare gas covered $\mathrm{Au}(111)$ surfaces. The presence of $\mathrm{Ar}, \mathrm{Kr}$, and Xe adsorbates on the surface enhances $\gamma$ in an unexpected way. $\gamma$ of around 0.5 is observed for monolayer coverage of all three adsorbates and electron emission efficiencies close to unity are observed for $\mathrm{Ar}$ and $\mathrm{Kr}$ coverages greater than 1 monolayer.

The unexpectedly high electron emission probability for $\mathrm{CO}^{*}(v=0)$ on $\mathrm{Au}(111)$, the vibrational enhancement of electron yield, and the adsorbate induced enhancement of electron emission efficiency can all be understood within the framework of an electron transfer mediated auto-detachment de-excitation mechanism. 


\section{Summary and outlook}

This work presents details of the design, construction, and characterization of a novel surface scattering apparatus used to perform quantum state resolved studies of electronically excited molecules quenching on surfaces. The machine combines for the first time a decelerator for neutral polar molecules with a state-of-the-art surface scattering setup. Using molecular beams coupled with spectroscopic methods, hexapole focusing, and Stark deceleration, intense pulses of carrier gas-free quantum state-selected $\mathrm{CO}$ can be produced with tunable translational energy and an extremely narrow distribution of velocities. The apparatus is equipped with devices for characterization of the atomic composition and electronic work function of the surface. The temperature of the $\mathrm{Au}(111)$ surface can be controlled from $19 \mathrm{~K}$ to the melting point of the crystal. Surface composition can be modified with atomic precision using targeted adsorbate dosing and thermal desorption techniques. Exoelectrons originating near the surface and laser ionized molecules are detected on dual microchannel plate detectors close to the surface in order to learn details about scattering processes.

Desorption activation energies and exponential prefactors were measured for $\mathrm{Ar}, \mathrm{Kr}, \mathrm{Xe}$, $\mathrm{CO}, \mathrm{NO}, \mathrm{N}_{2}, \mathrm{C}_{2} \mathrm{H}_{2}$, and $\mathrm{SF}_{6}$ adsorbates on $\mathrm{Au}(111)$ using thermal desorption methods. For these weakly bound species, the desorption activation energy is an excellent proxy for the adsorbate binding energy, which scales with the adsorbates' polarizability. This dependence indicates that the adsorbate-surface bond is dominated by dispersion forces. The kinetic parameters measured in this work will serve as a small library of benchmarks for emerging theory which attempts to accurately model dispersion forces acting between atoms/molecules and surfaces.

Further, we have investigated the mechanism by which metastable carbon monoxide molecules $\left(\mathrm{CO}^{*}\right)$ de-excite near a $\mathrm{Au}(111)$ surface and emit electrons into vacuum. We determined the absolute electron emission probability, $\gamma=0.13$ for $\mathrm{CO}^{*}(v=0) . \gamma$ increases with vibrational excitation of the incident molecule. Modifying the surface with $\mathrm{Ar}, \mathrm{Kr}$, and $\mathrm{Xe}$ adsorbates leads to a dramatic enhancement of electron emission probability with increasing coverage, peaking around 1.5 monolayers. Electron emission is suppressed at adsorbate coverage above 3 monolayers. 
The magnitude of $\gamma$ and the influence of vibrational excitation and surface composition on $\gamma$ are consistent with an anion-mediated (AM) auto-detachment mechanism. The mechanism proceeds in two steps. First, an electron transfers from the gold surface to $\mathrm{CO}^{*}$ as the molecule approaches the surface, forming an anion. Second, the anion relaxes to the ground electronic state of the neutral molecule on a femtosecond time scale by auto-detaching an electron. The electron emitted from the molecule can either escape into vacuum and be detected or be absorbed by the surface.

Vibrational enhancement of electron emission is reasonable within the framework of the AM model. The initial electron transfer step from the surface to the molecule can only occur when the molecule is at extended bond lengths. $\mathrm{CO} *$ molecules in higher vibrational states spend more time with extended C-O bond lengths, thereby increasing the efficiency of the first electron transfer step. This leads to ionization at greater molecule-surface distances. Therefore, the subsequently auto-detached electron experiences weaker image forces with the surface and has a higher probability of escaping into vacuum.

Adsorbate enhancement of $\gamma$ can be understood when one considers the increased electron reflectivity of closed-shell noble gases versus that of a metal surface. With increased adsorbate coverage, the probability that an electron emitted from the molecule toward the surface is scattered back into vacuum increases. After some critical adsorbate coverage, the initial electron transfer step from surface to molecule becomes inefficient and electron emission begins to decrease with additional adsorbate coverage.

Experiments planned for the near future will scatter ground electronic state $\mathrm{CO}$ in high vibrational quantum states from $\mathrm{Au}(111)$ that has been decorated with cesium to lower the work function. It is known that highly vibrationally excited NO can lose several quanta of vibrational energy to a single electron in the $\mathrm{Au}(111)$ surface. If the surface work function is lowered sufficiently, this energy exchange can lead to emission of electrons. Electron emission is enhanced when the incident molecule approaches the surface with low translational energy. The new apparatus is uniquely suited to investigation of similar phenomena that exhibit inverse velocity dependence, granting confidence that corresponding experiments using translationally cold CO will yield interesting and important results. 
Another long-term strength of the apparatus is its flexibility. In addition to a wide range of molecular beam and surface conditions, the surface can be easily replaced with a different target to make comparative measurements. Stark deceleration can also be performed on different molecules such as $\mathrm{OH}, \mathrm{NO}$, and $\mathrm{NH}_{3}$.

In conclusion, a new apparatus was constructed for scattering velocity controlled molecular beams from surfaces with complete control over molecular and surface degrees of freedom. The de-excitation of metastable CO molecules quenching on the Au(111) surface was found to proceed by an anion-mediated auto-detachment mechanism. The apparatus built during the course of this work is now fully characterized and ready to unlock the secrets of the universe. 


\section{Bibliography}

C. N. R. Rao and G. R. Rao, Surf. Sci. Rep. 13, 221 (1991).

M. Caragiu, G. S. Leatherman, T. Seyller, and R. D. Diehl, Surf. Sci. 475, 89 (2001).

F. Brunet, R. Schaub, S. Fedrigo, R. Monot, J. Buttet, and W. Harbich, Surf. Sci. 512, 201 (2002).

N. D. Lang, A. R. Williams, F. J. Himpsel, B. Reihl, and D. E. Eastman, Phys. Rev. B 26, 1728 (1982).

A. V. Bukhtiyarov, R. I. Kvon, A. V. Nartova, I. P. Prosvirin, and V. I. Bukhtiyarov, Surf. Sci. 606, 559 (2012).

S. Rohe, K. Frank, A. Schaefer, A. Wittstock, V. Zielasek, A. Rosenauer, and M. Baumer, Surf. Sci. 609, 106 (2013).

G. Ertl, Angewandte Chemie-International Edition in English 29, 1219 (1990).

H. D. Hagstrum, Physical Review 96, 336 (1954).

H. Conrad, G. Ertl, J. Kuppers, S. W. Wang, K. Gerard, and H. Haberland, Phys. Rev. Lett. 42, 1082 (1979).

H. Conrad, G. Ertl, J. Kuppers, W. Sesselmann, and B. Woratschek, Surf. Sci. 117, 98 (1982).

H. Conrad, G. Doyen, G. Ertl, J. Kuppers, W. Sesselmann, and H. Haberland, Chemical Physics Letters 88, 281 (1982).

B. Woratschek, W. Sesselmann, J. Kuppers, G. Ertl, and H. Haberland, Phys. Rev. Lett. 55, 1231 (1985).

H. D. Hagstrum, Physical Review 104, 309 (1956).

H. D. Hagstrum, Journal of Applied Physics 31, 897 (1960).

H. D. Hagstrum, Phys. Rev. Lett. 43, 1050 (1979).

H. D. Hagstrum, Journal of Vacuum Science \& Technology 20, 626 (1982).

H. D. Hagstrum, E. E. Chaban, and P. Petrie, J. Vac. Sci. Technol. A-Vac. Surf. Films 2, 841 (1984).

H. D. Hagstrum, P. Petrie, and E. E. Chaban, Phys. Rev. B 38, 10264 (1988).

J. A. Barker and D. J. Auerbach, Surf. Sci. Rep. 4, 1 (1984).

D. J. Auerbach, Science 294, 2488 (2001).

A. M. Wodtke, J. C. Tully, and D. J. Auerbach, Int. Rev. Phys. Chem. 23, 513 (2004).

I. Rahinov, R. Cooper, D. Matsiev, C. Bartels, D. J. Auerbach, and A. M. Wodtke, Phys. Chem. Chem. Phys. 13, 12680 (2011).

C. Bartels, R. Cooper, D. J. Auerbach, and A. M. Wodtke, Chem. Sci. 2, 1647 (2011).

J. D. White, J. Chen, D. Matsiev, D. J. Auerbach, and A. M. Wodtke, Nature 433, 503 (2005).

J. D. White, J. Chen, D. Matsiev, D. J. Auerbach, and A. M. Wodtke, J. Vac. Sci. Technol. A 23, 1085 (2005).

J. D. White, J. Chen, D. Matsiev, D. J. Auerbach, and A. M. Wodtke, J. Chem. Phys. 124 (2006).

N. H. Nahler, J. D. White, J. Larue, D. J. Auerbach, and A. M. Wodtke, Science 321, 1191 (2008).

I. Rahinov, R. Cooper, C. Yuan, X. M. Yang, D. J. Auerbach, and A. M. Wodtke, J. Chem. Phys. 129 (2008).

D. Matsiev, Z. S. Li, R. Cooper, I. Rahinov, C. Bartels, D. J. Auerbach, and A. M. Wodtke, Phys. Chem. Chem. Phys. 13, 8153 (2011).

R. Cooper, Z. S. Li, K. Golibrzuch, C. Bartels, I. Rahinov, D. J. Auerbach, and A. M. Wodtke, J. Chem. Phys. 137 (2012).

R. Cooper, et al., Angew. Chem.-Int. Edit. 51, 4954 (2012).

K. Golibrzuch, A. Kandratsenka, I. Rahinov, R. Cooper, D. J. Auerbach, A. M. Wodtke, and C. Bartels, Journal of Physical Chemistry A 117, 7091 (2013).

H. L. Bethlem, G. Berden, and G. Meijer, Phys. Rev. Lett. 83, 1558 (1999).

J. Stark, Annalen Der Physik 348, 965 (1914). 
H. L. Bethlem, F. M. H. Crompvoets, R. T. Jongma, S. Y. T. van de Meerakker, and G. Meijer, Physical Review A 65 (2002).

S. Y. T. van de Meerakker, P. H. M. Smeets, N. Vanhaecke, R. T. Jongma, and G. Meijer, Phys. Rev. Lett. 94 (2005).

S. Y. T. van de Meerakker, N. Vanhaecke, H. L. Bethlem, and G. Meijer, Physical Review A 71 (2005).

S. Y. T. van de Meerakker, N. Vanhaecke, H. L. Bethlem, and G. Meijer, Physical Review A 73 (2006).

J. J. Gilijamse, S. Hoekstra, S. A. Meek, M. Metsala, S. Y. T. van de Meerakker, G. Meijer, and G.

C. Groenenboom, J. Chem. Phys. 127 (2007).

S. Y. T. Van De Meerakker, H. L. Bethlem, and G. Meijer, Nature Physics 4, 595 (2008).

J. H. Blokland, J. Riedel, S. Putzke, B. G. Sartakov, G. C. Groenenboom, and G. Meijer, J. Chem. Phys. 135 (2011).

S. Y. T. van de Meerakker, H. L. Bethlem, N. Vanhaecke, and G. Meijer, Chem. Rev. 112, 4828 (2012).

C. E. Heiner, H. L. Bethlem, and G. Meijer, Phys. Chem. Chem. Phys. 8, 2666 (2006).

R. C. Stern, R. H. Gammon, M. E. Lesk, R. S. Freund, and Klempere.Wa, J. Chem. Phys. 52, 3467 (1970).

G. Scoles, Atomic and Molecular Beam Methods (Oxford University Press, 1988).

L. Velarde, D. P. Engelhart, D. Matsiev, J. LaRue, D. J. Auerbach, and A. M. Wodtke, Rev. Sci. Instrum. 81 (2010).

G. Herzberg, Spectra of Diatomic Molecules (Van Nostrand Reinhold, 1950).

F. Grätz, Dissertation, Freie Universität Berlin, 2014.

V. I. Veksler, Comptes Rendus (Dokaldy) de l'Academie Sciences de l'URSS 43, 329 (1944).

E. M. McMillan, Physical Review 68, 143 (1945).

K. Gubbels, G. Meijer, and B. Friedrich, Physical Review A 73 (2006).

J. Moore, Davis, CD, Coplan, MA, Building Scientific Apparatus, 4th Ed. (Cambridge University Press, Cambridge, 2009).

D. Menzel, H. Pfnur, and P. Feulner, Surf. Sci. 126, 374 (1983).

H. Schlichting and D. Menzel, Rev. Sci. Instrum. 64, 2013 (1993).

A. Klekamp and E. Umbach, Surf. Sci. 249, 75 (1991).

A. Zangwill, Physics at Surfaces (Cambridge University Press, 1988).

A. Gross, Theoretical Surface Science: A Microscopic Perspective, Second Edition, 2009).

G. A. Somorjai and Y. Li, Introduction to Surface Chemistry and Catalysis (John Wiley \& Sons, 2010).

H. Cho, R. P. McEachran, S. J. Buckman, D. M. Filipovic, V. Pejcev, B. P. Marinkovic, H. Tanaka, A. D. Stauffer, and E. C. Jung, J. Phys. B-At. Mol. Opt. Phys. 39, 3781 (2006).

M. J. Dresser, T. E. Madey, and J. T. Yates, Surf. Sci. 42, 533 (1974).

J. Unguris, L. W. Bruch, E. R. Moog, and M. B. Webb, Surf. Sci. 37, 415 (1979).

M. Wolfel, M. Rauh, and P. Wissmann, Fresenius Journal of Analytical Chemistry 346, 362 (1993).

D. Vanlabeke, J. M. Vigoureux, and P. Grossel, J. Chem. Phys. 86, 1632 (1987).

D. A. King, Surf. Sci. 47, 384 (1975).

R. M. Nix, Queen Mary, University of London London, http://www.chem.qmul.ac.uk/surfaces/scc/scat2 4.htm.

P. Feulner and D. Menzel, Surf. Sci. 154, 465 (1985).

D. S. Bethune, J. A. Barker, and C. T. Rettner, J. Chem. Phys. 92, 6847 (1990).

C. T. Rettner, D. J. Auerbach, and H. A. Michelsen, Phys. Rev. Lett. 68, 1164 (1992). 
J. A. Barker and C. T. Rettner, J. Chem. Phys. 97, 5844 (1992).

V. P. Zhdanov, Surf. Sci. Rep. 12, 183 (1991).

P. A. Redhead and P. A. Redhead, Vacuum 12, 203 (1962).

A. M. Dejong and J. W. Niemantsverdriet, Surf. Sci. 233, 355 (1990).

J. B. Miller, H. R. Siddiqui, S. M. Gates, J. N. Russell, J. T. Yates, J. C. Tully, and M. J. Cardillo, J. Chem. Phys. 87, 6725 (1987).

J. W. Niemantsverdriet, P. Dolle, K. Markert, and K. Wandelt, J. Vac. Sci. Technol. A-Vac. Surf. Films 5, 875 (1987).

P. Feulner and D. Menzel, Journal of Vacuum Science \& Technology 17, 662 (1980).

N. D. Lang and W. Kohn, Phys. Rev. B 3, 1215 (1971).

K. Jacobi, in Physics of Solid Surfaces, Subvolume A: Adsorbed Layers on Surfaces, 2002).

K. Wandelt, Appl. Surf. Sci. 111, 1 (1997).

T. Roman and A. Gross, Phys. Rev. Lett. 110 (2013).

J. L. LaRue, J. D. White, N. H. Nahler, Z. Liu, Y. Sun, P. A. Pianetta, D. J. Auerbach, and A. M. Wodtke, J. Chem. Phys. 129 (2008).

I. D. Baikie, K. O. Vanderwerf, H. Oerbekke, J. Broeze, and A. Vansilfhout, Rev. Sci. Instrum. 60, 930 (1989).

I. D. Baikie, S. Mackenzie, P. J. Z. Estrup, and J. A. Meyer, Rev. Sci. Instrum. 62, 1326 (1991).

F. Graetz, D. P. Engelhart, R. J. V. Wagner, H. Haak, G. Meijer, A. M. Wodtke, and T. Schaefer, Phys. Chem. Chem. Phys. 15, 14951 (2013).

A. Tkatchenko, L. Romaner, O. T. Hofmann, E. Zojer, C. Ambrosch-Draxl, and M. Scheffler, Mrs Bulletin 35, 435 (2010).

S. Ehrlich, J. Moellmann, and S. Grimme, Accounts Chem. Res. 46, 916 (2013).

A. J. Cohen, P. Mori-Sanchez, and W. Yang, Chem. Rev. 112, 289 (2012).

J. Klimes and A. Michaelides, J. Chem. Phys. 137 (2012).

W. Liu, A. Tkatchenko, and M. Scheffler, Accounts Chem. Res. 47, 3369 (2014).

A. M. Wodtke, D. Matsiev, and D. J. Auerbach, Prog. Surf. Sci. 83, 167 (2008).

S. M. Janke, M. Pavanello, G.-J. Kroes, D. Auerbach, A. M. Wodtke, and A. Kandratsenka, Zeitschrift Fur Physikalische Chemie-International Journal of Research in Physical Chemistry \& Chemical Physics 227, 1467 (2013).

K. Golibrzuch, N. Bartels, D. J. Auerbach, and A. M. Wodtke, Annu. Rev. Phys. Chem. (2014).

N. Bartels, B. C. Kruger, D. J. Auerbach, A. M. Wodtke, and T. Schafer, Angew. Chem.-Int. Edit. 53, 13690 (2014).

P. R. Shirhatti, J. Werdecker, K. Golibrzuch, A. M. Wodtke, and C. Bartels, J. Chem. Phys. 141 (2014).

F. Graetz, D. P. Engelhart, R. J. V. Wagner, G. Meijer, A. M. Wodtke, and T. Schaefer, J. Chem. Phys. 141 (2014).

Y. H. Huang, C. T. Rettner, D. J. Auerbach, and A. M. Wodtke, Science 290, 111 (2000).

N. Bartels, K. Golibrzuch, C. Bartels, L. Chen, D. J. Auerbach, A. M. Wodtke, and T. Schäfer, Proceedings of the National Academy of Sciences 110, 17738 (2013).

P. R. Shirhatti, J. Werdecker, K. Golibrzuch, A. M. Wodtke, and C. Bartels, J. Chem. Phys. 141, 124704 (8 pp.) (2014).

B. C. Krüger, N. Bartels, C. Bartels, A. Kandratsenka, J. C. Tully, A. M. Wodtke, and T. Schäfer, The Journal of Physical Chemistry C 119, 3268 (2015).

H. Schlichting and D. Menzel, Surf. Sci. 272, 27 (1992).

S. M. McClure, T. S. Kim, J. D. Stiehl, P. L. Tanaka, and C. B. Mullins, J. Phys. Chem. B 108, 17952 (2004).

J. L. Falconer and R. J. Madix, Surf. Sci. 48, 393 (1975). 
W. M. Haynes, CRC Handbook of Chemistry and Physics, 95th Edition (Taylor \& Francis, 2014). W. A. Brown, P. Gardner, M. P. Jigato, and D. A. King, J. Chem. Phys. 102, 7277 (1995).

E. M. Stuve, R. J. Madix, and B. A. Sexton, Surf. Sci. 123, 491 (1982).

B. C. Stipe, M. A. Rezaei, and W. Ho, Science 280, 1732 (1998).

M. J. Dresser, T. E. Madey, and J. T. Yates Jr, Surf. Sci. 42, 533 (1974).

F. M. Penning, Naturwissenschaften 15, 818 (1927).

Y. Harada, S. Masuda, and H. Ozaki, Chem. Rev. 97, 1897 (1997).

J. C. Tully, Phys. Rev. B 16, 4324 (1977).

J. W. Gadzuk and J. K. Norskov, J. Chem. Phys. 81, 2828 (1984).

N. Lorente, D. Teillet-Billy, and J. P. Gauyacq, Surf. Sci. 432, 155 (1999).

P. Stracke, F. Wiegershaus, S. Krischok, and V. Kempter, Surf. Sci. 396, 212 (1998).

J. Marbach, F. X. Bronold, and H. Fehske, Phys. Rev. B 84 (2011).

J. Marbach, F. X. Bronold, and H. Fehske, Phys. Rev. B 86 (2012).

J. Marbach, F. X. Bronold, and H. Fehske, European Physical Journal D 66 (2012).

D. P. Engelhart, R. J. V. Wagner, P. C. Johnsen, A. M. Wodtke, and T. Schafer, Phys. Chem. Chem. Phys. 17, 11540 (2015).

Appelbau.Ja and D. R. Hamann, Phys. Rev. B 6, 1122 (1972).

N. V. Smith, C. T. Chen, and M. Weinert, Phys. Rev. B 40, 7565 (1989).

A. J. Smith, R. E. Imhof, and F. H. Read, J. Phys. B-At. Mol. Opt. Phys. 6, 1333 (1973).

H. L. Bethlem, G. Berden, A. J. A. van Roij, F. M. H. Crompvoets, and G. Meijer, Phys. Rev. Lett. 84, 5744 (2000).

S. Schohl, H. A. J. Meijer, M. W. Ruf, and H. Hotop, Meas. Sci. Technol. 3, 544 (1992).

C. V. V. Prasad, G. L. Bhale, and S. P. Reddy, J. Mol. Spectrosc. 121, 261 (1987).

M. Zubek, Chemical Physics Letters 149, 24 (1988).

J. Zobel, U. Mayer, K. Jung, and H. Ehrhardt, J. Phys. B-At. Mol. Opt. Phys. 29, 813 (1996).

L. A. Morgan and J. Tennyson, J. Phys. B-At. Mol. Opt. Phys. 26, 2429 (1993).

V. Laporta, C. M. Cassidy, J. Tennyson, and R. Celiberto, Plasma Sources Science \& Technology 21 (2012).

M. Allan, Physical Review A 81 (2010).

J. L. LaRue, T. Schafer, D. Matsiev, L. Velarde, N. H. Nahler, D. J. Auerbach, and A. M. Wodtke, Journal of Physical Chemistry A 115, 14306 (2011).

C. Huckstadt, S. Schmidt, S. Hufner, F. Forster, F. Reinert, and M. Springborg, Phys. Rev. B 73 (2006).

D. P. Engelhart, F. Grätz, R. J. V. Wagner, H. Haak, G. Meijer, A. M. Wodtke, and T. Schäfer, Rev. Sci. Instrum. 86, 043306 (2015).

D. L. Chen, W. A. Al-Saidi, and J. K. Johnson, Phys. Rev. B 84 (2011).

D.-L. Chen, W. A. Al-Saidi, and J. K. Johnson, Journal of Physics-Condensed Matter 24 (2012).

J. L. F. Da Silva and C. Stampfl, Phys. Rev. B 77 (2008).

R. D. Diehl, T. Seyller, M. Caragiu, G. S. Leatherman, N. Ferralis, K. Pussi, P. Kaukasoina, and M. Lindroos, Journal of Physics-Condensed Matter 16, S2839 (2004).

Y. Sonnenblick, E. Alexander, Z. H. Kalman, and I. T. Steinberger, Chemical Physics Letters 52, 276 (1977).

H. J. Herlt and E. Bauer, Surf. Sci. 175, 336 (1986).

H. Haberland, T. Kolar, and T. Reiners, Phys. Rev. Lett. 63, 1219 (1989).

P. Stampfli, Physics Reports-Review Section of Physics Letters 255, 1 (1995).

http://www.thinksrs.com/downloads/labVIEW.htm), RGA LabVIEW Development Kit. 


\section{Appendix}

Heating the surface in a linear fashion is accomplished using a proportional feedback loop written using the graphical programming platform LabVIEW. Briefly, surface heating begins using a chosen heating current, and temperature is measured as a function of time. The heating rate is calculated and compared to the target heating rate, and a correction is applied to the heating current depending on the divergence of the actual rate from the chosen rate and an empirically determined feedback constant. At each iteration of the feedback loop, partial pressure for a chosen mass-to-charge is logged versus surface temperature. Details of the program are presented in the following figures. 


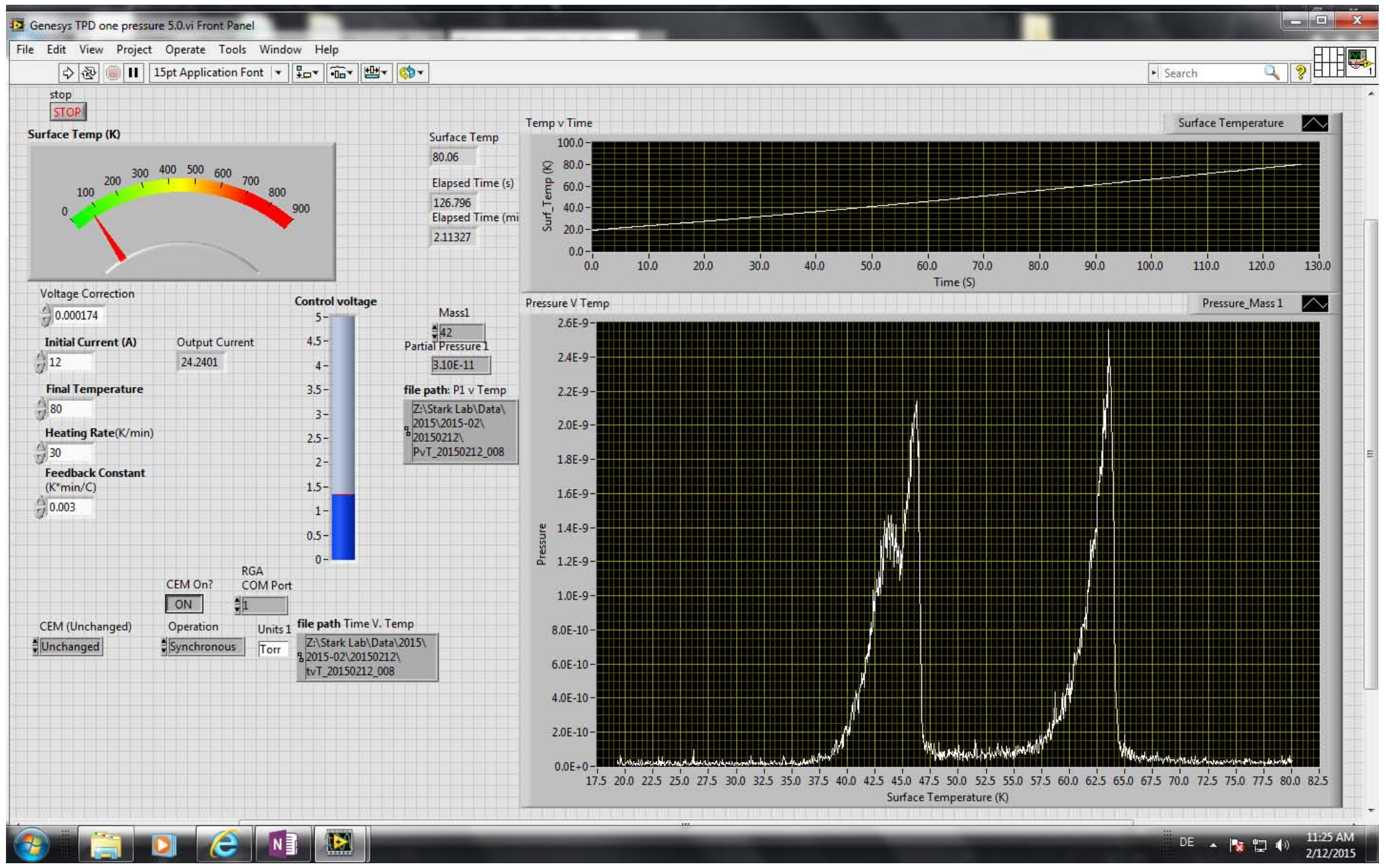

Figure 54 Front panel of the LabVIEW program "TPD one pressure 5.0.vi." Voltage Correction, Initial Current, Final Temperature, Heating Rate, Feedback Constant, Mass1, RGA COM port, Operation, CEM on?, CEM (unchanged) and both file path boxes are user defined control parameters. All other boxes, gauges, and graphs are indicators returned from the program during operation. 


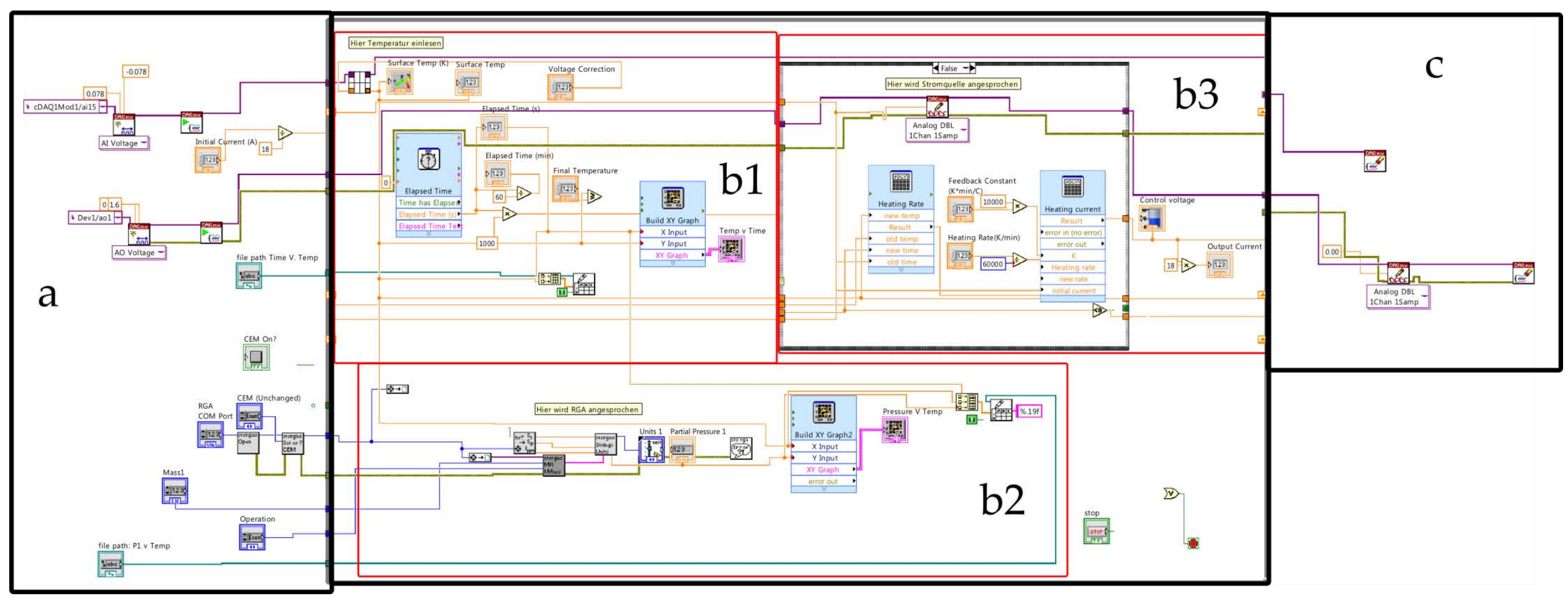

Figure 55 Block diagram of the LabVIEW program "TPD one pressure 5.0.vi" used to control the surface heating rate using a proportional feedback loop. In section a, all user inputs are defined and communication with the RGA and heating power supply are established. Section b comprises the feedback loop. Surface temperature versus time is measured and plotted in section b1 and sent to section b3. Partial pressure versus surface temperature is recorded and plotted in section b2. The heating rate and heating voltage correction are calculated and voltage is adjusted in section b3. Section c ends the program and returns heating voltage to zero and discontinues communication between the computer and the mass analyzer. See the following pages for details of each section. 


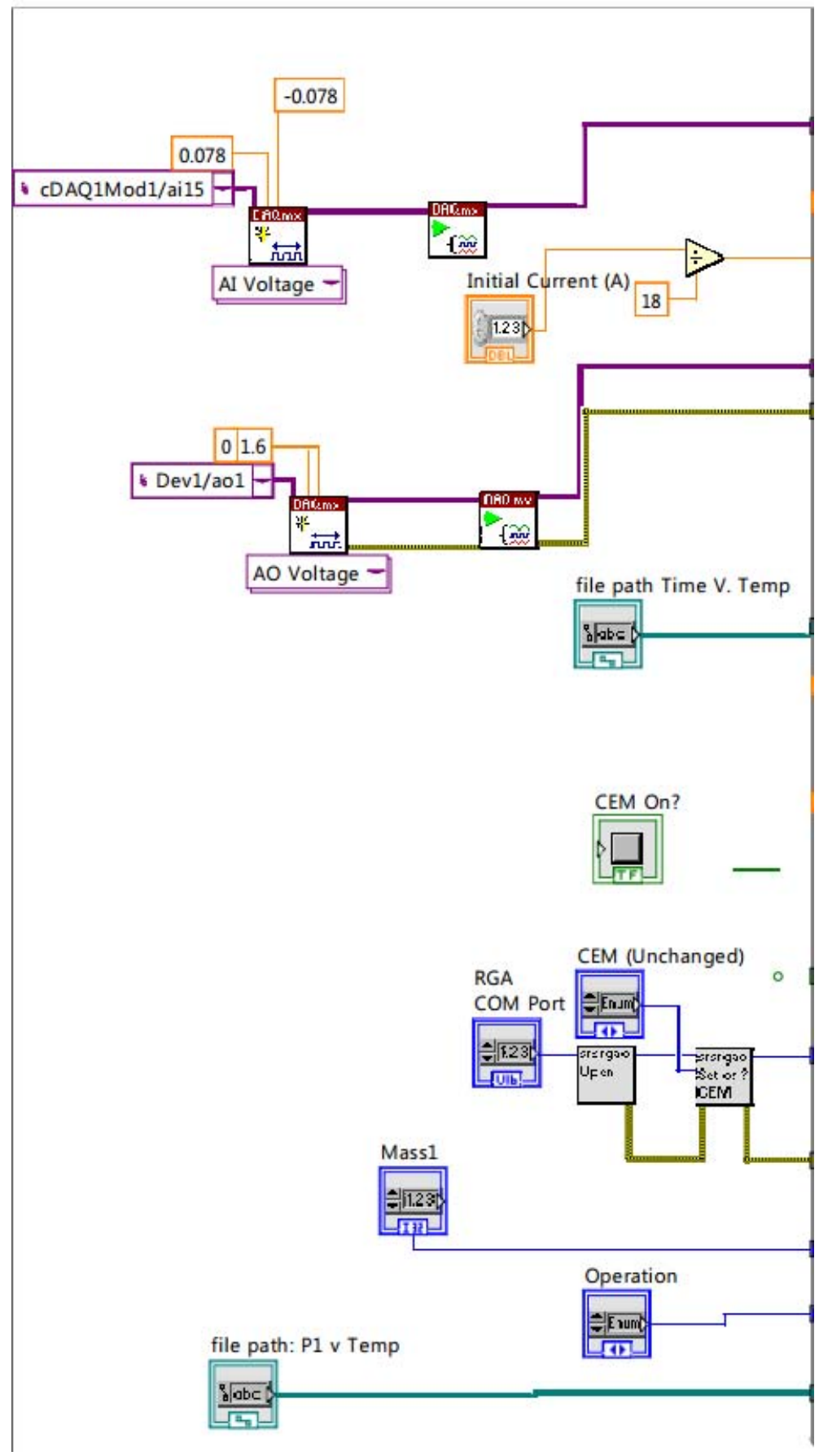

Figure 56 Section a of Figure 55. Initial parameters are entered by the user before the program begins. The A0 voltage subVI sets the minimum and maximum control voltages that can be sent via an analog to digital converter (ADC) to the power supply. This parameter can only be changed in the block diagram as there is no corresponding control on the front panel. A heating current of 0-180 A corresponds to a 0-10 V control voltage. The A1 Voltage subVI defines the input channel for the e-type thermocouple voltage which is fed to the PC via a NI-9213 thermocouple input. CEM, RGA COM port, and Operation are all subVIs for initializing communication with and controlling the residual gas analyzer (RGA) and were acquired from Stanford Research Systems. ${ }^{139}$ 


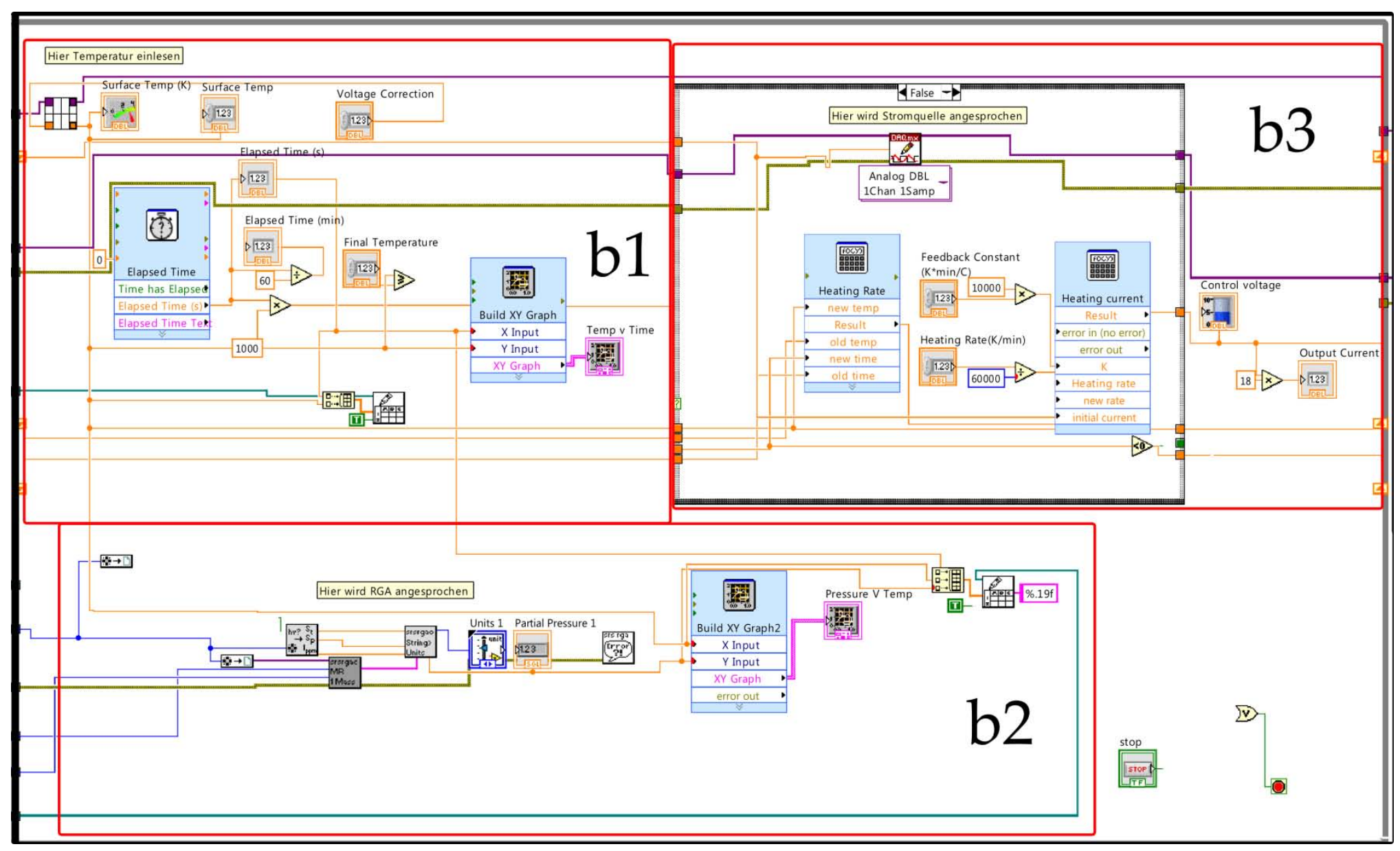

Figure 57 Section $b$ is a while loop in which surface temperature and partial pressure are monitored and the surface heating rate is controlled. In section b1, thermocouple voltage is converted to temperature and recorded as a function of time. This information is sent to section b3. In section b2, partial pressure is recorded as a function of surface temperature. See the next figure for information about section b3. The practical upper limit to iteration frequency is dependent on the communication between PC and RGA/heating current power supply and is approximately $10 \mathrm{~Hz}$. 


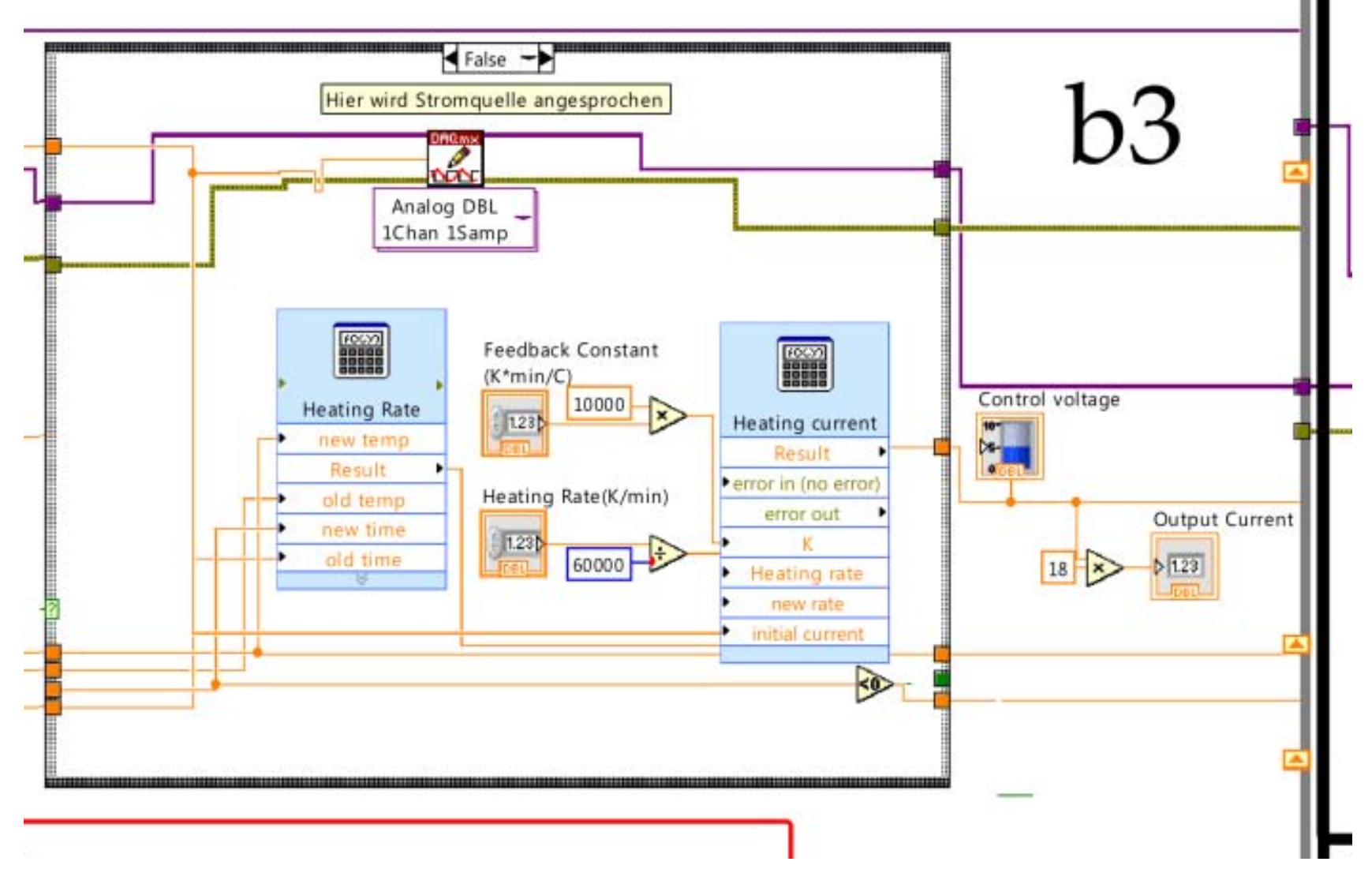

Figure 58 The majority of section b3 is set within a case structure (left). The heating rate is calculated and compared to the chosen heating rate and a correction to the heating current is calculated based on the user defined feedback constant as discussed in section 2.2.1.2. The corresponding command voltage is sent to the heating current source via an external ADC. The case structure is defined such that if the surface temperature is lower than the chosen final temperature, time and temperature information is written into the shift register as starting information for the next iteration of section $b$ and the loop begins again. If the surface temperature is greater than the user defined final temperature, the stop condition is met and the program proceeds to section $\mathrm{c}$. 


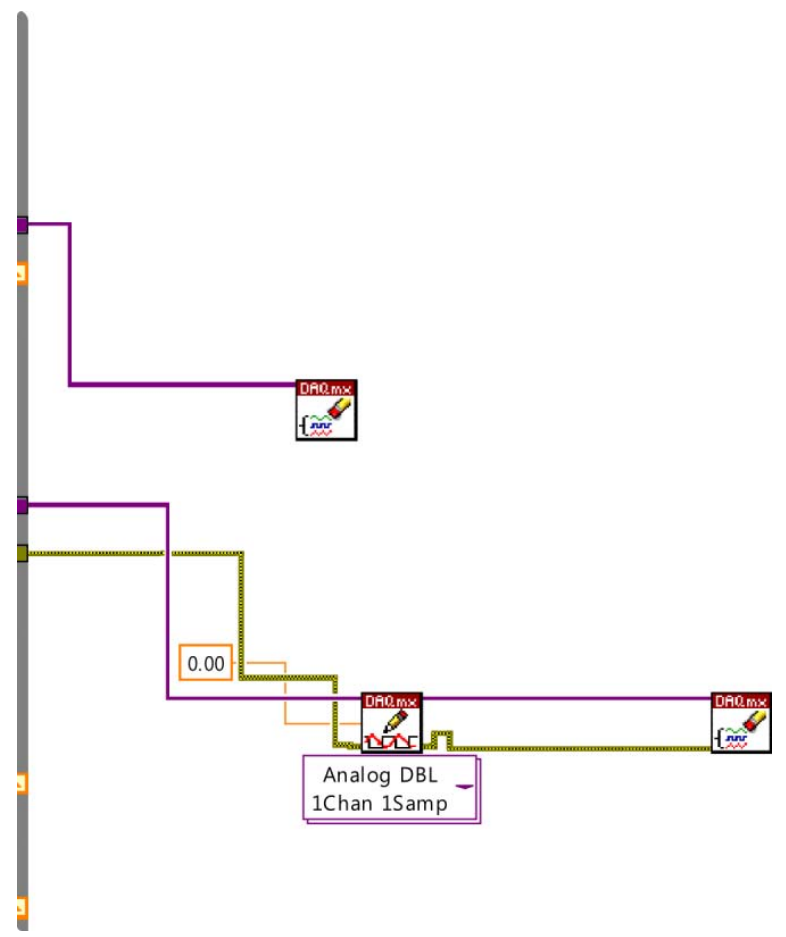

Figure 59 Section $\mathrm{c}$ is accessed only after the while loop has met its stop criterion, at which point, the surface heating power supply is set to null output and the RGA is set to standby mode. 


\section{Acknowledgements}

I would like to thank Alec Wodtke, Gerard Meijer, and Dan Auerbach for their support and motivation over the last several years. Your creativity and sense of wonder coupled perfectly with a healthy dose of skepticism to provide a keen and collaborative working environment. You have allowed me to live the life of a gentleman scientist for a few years. Thank you.

Thank you, Tim, for your endless enthusiasm and your hard work. You have been my teacher and my boon companion. I will take home fond memories of working, grilling, and rock and roll. You made me part of your family. You and your brood will have to visit Uncle Dan sometime soon. Fabian, we did it! The long hours in the lab, the discussion and head-scratching, the moving, and all the push-ups just to stay sane have all been worth it. You're a good friend and I'm happy that we built this monster together. Roman Wagner, it's been a pleasure to get to know you and I'm glad to leave the apparatus in such capable hands. I know you'll treat her well.

The work presented in this thesis would not be possible without the support of such a great team. Discussions with Luis Velarde and Daniel Matsiev back at UCSB got the ball rolling. In retrospect, I should have listened better. Igor Rahinov, your musings on the academic life have been invaluable. And the fact that you quit smoking proved that anything is possible if you put your mind to it.

Thank you Bruce, Terry, and Mike in the UCSB machine shop, Petrik and Henrik in the FHI workshop, and GU workshops. Christian, Alex, Laura, Schewe, Bas, Isabel, Sam, Moritz, Gert, Inge, and Andrea at the Fritz; Hongyan, Pranav, Russ, Nils, Dan, Basti, Christof, Theo, Jannis, Oliver, Kai, Svenja, Artur, the Heartbreakers, and all the other members of the group in Göttingen, thanks for everything. Dirk and Jörg, thank you for your constant support.

Jürgen Troe, our late-night talks in the office are a constant source of entertainment. You've done it all and you have proven to me that working hard is the most important thing. Arne, thanks for help with my golf swing and anger management (in no particular order). Sascha and Angelika, thank you for the palmeni and so much more. The world will not save itself. Florian, my friend, you took me in as one of your own. I look forward to repaying the favor. 
Thank you to all the people I've met along the way who have made me who I am. Terrance, Josh, Dave, Scott, Jason, and everybody else back in Harpswell, scooping rotten bait out of a bucket at 4 a.m. and still not catching any lobsters makes a bad day in the lab seem like a cakewalk. After spending 4 years on a boat, one knows deep in their bones that we are all at the mercy of physical forces beyond our control. This is a vital lesson for an experimentalist. Trent, Larry, and Carl at Allied, considering the number of times I've moved myself and others during this project, you should all be on my committee. Bob and Brian back at Taylor Music, yours was the first workshop I ever worked in. It was so much fun, I didn't want to stop. I just moved from hammers and torches to manipulators and lasers.

I would also like to thank my family. In many ways, the last few years have been a rough road. We pulled together when times were tough and I drew so much strength from all of you, even from so far away. Barb, Jolane and Dennis, Diane and Tim, Joey and Sharon, Allison, Austin and Brigitte, Taylor, Cecily, Alexis, Bailey, Lisa, Beth, baby Zakura and all assorted hangers-on, thank you. It would not have been possible without you.

Finally, I would like to thank my father, Joseph, and my mother, Ellen, for giving me all the tools I need to survive and thrive in this big weird world. This work is dedicated to both of you. 


\section{Lebenslauf}

I, Daniel Paul Engelhart, was born on October $28^{\text {th }}, 1978$ to Joseph P. and Ellen M. Engelhart in Aberdeen, South Dakota, USA. I am a citizen of the United States of America.

EDUCATION

09.2010 - Present

Max-Planck-Institute for Biophysical Chemistry, Göttingen, Germany

Fritz-Haber Institute of the Max Planck Society, Berlin, Germany

Master of Science, Physical Chemistry

09.2008 - 08.2010

University of California, Santa Barbara: "An Apparatus for Gas-Surface Scattering Studies of Stark-Decelerated CO”

Bachelor of Arts, Chemistry and German

09.2005 - 12.2007, 09.1997- 05.2002

University of South Dakota, Vermillion

\section{PROFESSIONAL EXPERIENCE}

\section{Research Assistant}

01.2008 - 08.2008, University of South Dakota, Vermillion, South Dakota

\section{Salesman}

01.2006 - 08.2007, Cingular Wireless, Vermillion, South Dakota

\section{Certified Nurse's Assistant}

01.2005 - 08.2005, Pleasant Meadows Christian Village Nursing Home, Chrisman, Illinois

\section{Professional Lobster Fisherman}

05.2002 - 12.2004, Harpswell, Maine

\section{Furniture Mover}

2000-2006 (seasonal), Jobber's Moving and Storage, Allied Van Lines, Aberdeen, South Dakota 